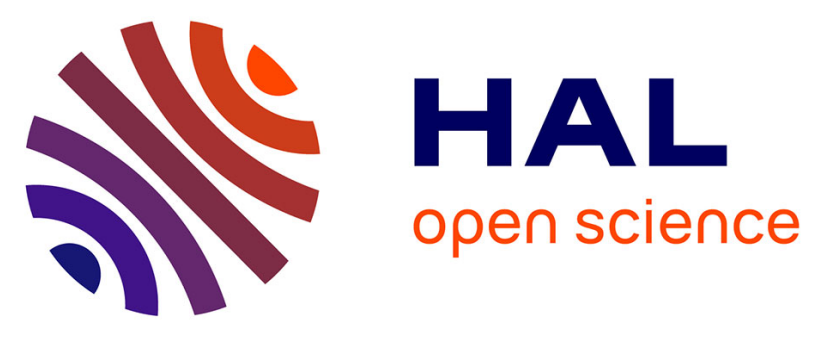

\title{
Trois cimetières ruraux de l'Antiquité tardive dans la moyenne vallée du Rhône: les sites du Pillon à Marennes (Rhône), du Trillet à Meyzieu (Rhône) et des Girardes à Lapalud (Vaucluse)
}

Frédérique Blaizot, Christine Bonnet, Dominique Castex, Henri Duday, Christian Cécillon, Daniel Frascone, Ghislaine Macabéo, Karine Roger, Magali Rolland, Serge Martin, et al.

\section{- To cite this version:}

Frédérique Blaizot, Christine Bonnet, Dominique Castex, Henri Duday, Christian Cécillon, et al.. Trois cimetières ruraux de l'Antiquité tardive dans la moyenne vallée du Rhône: les sites du Pillon à Marennes (Rhône), du Trillet à Meyzieu (Rhône) et des Girardes à Lapalud (Vaucluse). Gallia Archéologie de la France antique, 2001, 58, pp.271-361. 10.3406/galia.2001.3028 • hal-01909318

\author{
HAL Id: hal-01909318 \\ https://hal.science/hal-01909318
}

Submitted on 16 Jan 2020

HAL is a multi-disciplinary open access archive for the deposit and dissemination of scientific research documents, whether they are published or not. The documents may come from teaching and research institutions in France or abroad, or from public or private research centers.
L'archive ouverte pluridisciplinaire HAL, est destinée au dépôt et à la diffusion de documents scientifiques de niveau recherche, publiés ou non, émanant des établissements d'enseignement et de recherche français ou étrangers, des laboratoires publics ou privés.

\section{(ㅇ)(1) $\$$}

Distributed under a Creative Commons Attribution - NonCommercial - NoDerivatives 44.0 


\title{
TROIS CIMETIÈRES RURAUX DE L'ANTIQUITÉ TARDIVE DANS LA MOYENNE VALLÉE DU RHÔNE
}

\author{
Les sites du Pillon à Marennes (Rhône), du Trillet à Meyzieu \\ (Rhône) et des Girardes à Lapalud (Vaucluse)
}

Frédérique Blaizot, Christine Bonnet, Dominique Castex et Henri DudaY Avec la collaboration de Christian CÉCIllon, Daniel Frascone, Ghislaine MaCABÉo, Karine Roger, Magali Rolland

Et les contributions de Serge Martin, Luc StaniASZEK, Abdelweded BENNcer

Mots-clés. Antiquité tardive, pratiques funéraires, petits établissements ruraux, paléodémographie, vallée du Rhône.

Key-words. Late Antiquity, burial practices, little rural settlements, paleodemography, Rhône valley.

Résumé. Cette étude concerne trois siles funéraires ruraux de la moyenne vallée du Rhône appartenant à une même période chronologique, l'Antiquité tardive. Fouillés de manière exhaustive, ils offrent tous trois la particularité de présenter un petit nombre de sépultures en relation avec un établissement domestique. L'analyse interne à chaque gisement s'appuie sur une prise en compte globale des faits tant archéologiques que biologiques, de manière à mettre en évidence les caractéristiques propres à chacun de ces petits ensembles. En dépit de leur caractère parfois lacunaire, les données recueillies sur les structures des tombes, les modes d'inhumation et le mobilier funéraire, croisées avec l'étude démographique des squelettes, nous ont permis de mieux comprendre les modalités de "recrutement " et d'organisation de chaque ensemble étudié, les constantes et les différences qui les caractérisent et d'élargir le champ de recherche à une problématique générale concernant la période tardo-antique.

Abstract. This study is based on three rural burial areas of the Rhône middle valley which belong to the same chronological period, Late Antiquity. These funerary sites have been systematically excavated and their originality is the relationship between some of the burials and domestic buildings. An internal analysis of each site is supported by an overall view of both archaeological and biological facts in order to improve the specificity of the sites being studied. Despite a paucity of evidence, observations concerning tomb architecture, methods of inhumation and grave goods, in combination with paleodemographic study, have played a great part to a better understanding of the selting and organization of each funerary site. Results add informations and answers to the general problems related to the Late Antiquity. 
Abdelweded BenNcer, Musée national des Antiquités, INSAP, Rabat, Maroc.

Frédérique BLAIzoT, AFAN-Lyon, 12, rue Maggiorini, F-69500 Bron.

Christine BonNer, AFAN-Lyon, 12, rue Maggiorini, F-69500 Bron.

Dominique CAstex, UMR 5809 du CNRS, Université de Bordeaux I, Avenue des Facultés, F-33405 Talence cedex.

Christian CÉCILLON, AFAN-Lyon, 12, rue Maggiorini, F-69500 Bron.

Henri DudAY, UMR 5809 du CNRS, Université de Bordeaux I, Avenue des Facultés, F-33405 Talence cedex.

Daniel Frasconk, AFAN-Lyon, 12, rue Maggiorini, F-69500 Bron.

Ghislaine MACABÉO, AFAN-Lyon, 12, rue Maggiorini, F-69500 Bron.

Serge MARTIN, AFAN-Lyon, 12, rue Maggiorini, F-69500 Bron.

Karine ROGER, AFAN-Nîmes, 12 rue Régale, F-30000 Nîmes.

Magali RoI..AND, AFAN-Lyon, 12, rue Maggiorini, F-69500 Bron.

Luc STANIASZEK, AFAN-Metz, 41 route de Jouy, F-57160 Moulins-lès-Metz.

\section{PROBLÉMATIQUE ET OBJECTIFS}

Malgré le nombre croissant d'opérations archéologiques, peu d'ensembles funéraires ruraux du BasEmpire font l'objet d'une étude exhaustive. Les synthèses réalisées s'appuient souvent sur des sites partiellement fouillés et ne prennent pas toujours en compte la totalité des données, souvent anciennes et insuffisamment exploitées. Les principales orientations définies en France et ailleurs pour l'étude des cimetières de l'Antiquité tardive se sont tout d'abord inscrites dans une tradition diffusionniste, puisque étaient surtout abordés les problèmes relatifs à la christianisation (Watts, 1991, p. 38-98 ; Boiron, 1993 ; Manniez, 1993) et à l'implantation des « populations barbares ", qui ont donné lieu à de grandes études morphologiques des séries de notre région (programme $\mathrm{H} 2$ : « Histoire du peuplement de la vallée du Rhône ", coordonnateurs : L. Buchet, P. Porte, 1986). Les perspectives " socio-économiques » et "politico-idéologiques", représentatives de la recherche anglo-saxonne (Philpott, 1993 ; Morris, 1995 ; Bintliff, Hamerow, 1995) ont été peu développées en France, la question étant effleurée mais non traitée, bien qu'elle serve d'explication générale (Carru et al., 1991, p. 53-55 ; Colardelle et al., 1996, p. 296).

À notre connaissance, il existe quatre synthèses pour le milieu rural. Deux d'entre elles, qui concernent le Languedoc et la vallée du Rhône, posent les bases d'une analyse spécifique aux sépultures et aux nécropoles (Colardelle, 1983 ; Raynaud, 1987; Colardelle et al., 1996) ; elles s'attachent à approcher globalement la typo- chronologie des sépultures et les coutumes funéraires. La première amorce une réflexion dans laquelle la nécropole est placée dans son environnement. Dans les deux autres synthèses, la problématique est orientée sur les modalités de l'occupation des sols ; la première a été réalisée pour le nord (Van Ossel, 1992), tandis que la seconde effectue un bilan plus large de la question pour l'ensemble de l'Antiquité sur une aire géographique plus étendue (Ferdière dir., 1993). Cette analyse est actuellement privilégiée, puisque depuis peu les ensembles funéraires sont résolument abordés dans le cadre d'une approche plus générale portant sur la dynamique régionale du peuplement (Ferdière dir., 1993 ; Brulet, 1993 ; Van Ossel, 1992, 1993 ; Gébara, Pasqualini, $1993 \ldots)$ qui se révèle la problématique actuelle principale pour l'Antiquité (Ouzoulias et al. dir., 2001).

Dans le monde rural, on observe fréquemment une discontinuité entre les lieux funéraires du Haut-Empire et ceux du Bas-Empire ; les cas où l'occupation funéraire est continue (Girard, 1997; Ferber, à paraitre) restent rares, contrairement à ce qui se passe en contexte urbain (Tranoy, 1995). Le moment auquel se produit cette séparation paraît se situer au cours du III $^{\mathrm{e}} \mathbf{s}$., mais le déficit général en tombes de cette période (Bel, Manniez, 1996), probablement relatif à des difficultés d'identification du fait de la raréfaction du mobilier dès le $\mathrm{II}^{\mathrm{e}} \mathrm{s}$., ne permet pas toujours de le préciser. À partir des III ${ }^{\mathrm{e}}-\mathrm{IV}^{\mathrm{e}} \mathrm{s}$., deux systèmes peuvent être distingués dans la topographie funéraire rurale. Dans le premier, apparaissent de véritables nécropoles à inhumation dont les sépultures s'organisent plus ou moins régulièrement, parfois 
en rangées ; elles s'établissent en rase campagne ou sur une villa en partie abandonnée, et peuvent perdurer jusque dans les siècles suivants (Vicherd, Baudrand, 1982 ; Feugère et al., 1987; Raynaud, 1987), parfois même jusqu'au début de la période carolingienne (Ginouvès, Schneider, 1987, p. 98, sites de Saint-Fréjus à Marennes et de La Côte à Ambronay dans l'Ain ; études inédites ; site du Patis à Montboucher-sur-Jabron dans la Drôme : Blaizot, à paraître). Le second système concerne des petits groupes de sépultures d'une durée de vie relativement courte, qui s'installent en bordure ou à distance des unités d'exploitation (Blasco et al., 1987 ; Ginouvès, Schneider, 1987, p. 96 ; Van Ossel, 1992), voire sur la partie ruinée d'un habitat qui subsiste à proximité (Ginouvès, Schneider, 1987, p. 93 et 96 ). Ces ensembles restent peu étendus, mais comportent un nombre de tombes généralement supérieur à dix, donc plus important que ceux du Haut-Empire qui, en dehors des agglomérations secondaires, sont peu denses (Bel et al., 1991). On peut alors supposer que la gestion des espaces funéraires se modifie; en se fixant pour aboutir à une occupation de plus longue durée, ils témoignent peutêtre d'un " recrutement " plus large qui dépasse l'individu ou la famille nucléaire.

Pour certains, la coexistence de ces deux modèles traduit, d'une part, la permanence d'une organisation "familiale » fréquemment attestée au Haut-Empire et, d'autre part, l'apparition d'un système " communautaire » (Carru et al., 1991, p. 53) ; le second est même, par extension, perçu comme la manifestation d'un contrôle centralisé des cimetières, puisqu'en Angleterre on aurait montré que ces ensembles ont été utilisés par plusieurs communautés adjacentes (Philpott, 1993, p. 416). Des dernières synthèses, il ressort une grande variété typologique de l'habitat tardo-antique (Ouzoulias et al. dir., 2001). Le même phénomène étant constaté pour les ensembles funéraires, il était légitime de tenter d'associer ces différentes formes d'habitat et de nécropoles (Van Ossel, 1992, p. 101). Or, si la coïncidence entre les formes d'habitat (groupé, dispersé) et celles des nécropoles est envisagée, elle ne peut pas toujours être démontrée. En effet, il reste très difficile de relier les sépultures à une occupation domestique en raison des contraintes de la limitation en surface des investigations archéologiques, de la distance plus ou moins importante qui sépare les sépultures et l'habitat et des difficultés que l'on rencontre à dater précisément les tombes. Si la
" contemporanéité " des occupations peut être globalement attestée, leur "synchronie " est plus difficile à mettre en évidence. Dans les cas où leur relation a pu être établie, on remarque qu'il existe de nombreux exemples où l'ensemble funéraire continue à être utilisé, tandis que la nature de l'occupation s'est modifiée (Van Ossel, 1993, p. 192). Des occupations synchrones ont cependant été mises en évidence et ont montré que là où le mode d'occupation est de tradition antique (petits ensembles en périphérie d'un habitat) les sépultures ont des caractères " romains " affirmés, alors que dans les grandes nécropoles, les pratiques funéraires, perçues à travers le type et l'agencement du mobilier, paraissent incontestablement "moins traditionnelles " (Carru et al., 1991 ; Van Ossel, 1992, p. 102 ; Philpott, 1993, p. 416-417).

De manière générale, l'interprétation de ces différents types d'ensembles funéraires repose surtout sur la perception directe que l'on a d'un certain nombre de leurs caractères (nature du mobilier, orientation et organisation des fosses, nombre de tombes, situation topographique...) et moins sur l'analyse de la relation des divers paramètres. Il n'est par exemple pas si courant que les informations propres à la sépulture, au squelette et au traitement du mobilier, entrent pleinement dans la discussion. De ce fait, la relation entre ce que l'on perçoit d'une organisation générale des vestiges et les références théoriques qui nous servent d'explications n'est pas toujours fondée sur l'analyse de toutes les données potentielles. Par exemple, l'hypothèse de petits groupes familiaux ne repose jamais sur l'analyse préalable de la distribution par sexe et par âge ; les pratiques funéraires restent très globalement définies (approximations dans la restitution des architectures, dans la mise en évidence des gestes funéraires, dans le traitement différentiel des individus...) et ne sont pas systématiquement analysées en fonction du type de nécropole auquel elles se rapportent.

Les trois sites présentés ici illustrent des cas rares où un petit ensemble funéraire, fouillé dans son intégralité, peut être mis en relation topographique directe avec un établissement domestique (fig. 1). Il s'agit des seuls exemples recensés pour la moyenne vallée du Rhône. L'objectif de cette étude, abordée à la fois par les méthodes de l'archéologie et de l'anthropologie biologique malgré l'état de conservation médiocre de certains squelettes, est de mettre en évidence les caracté- 

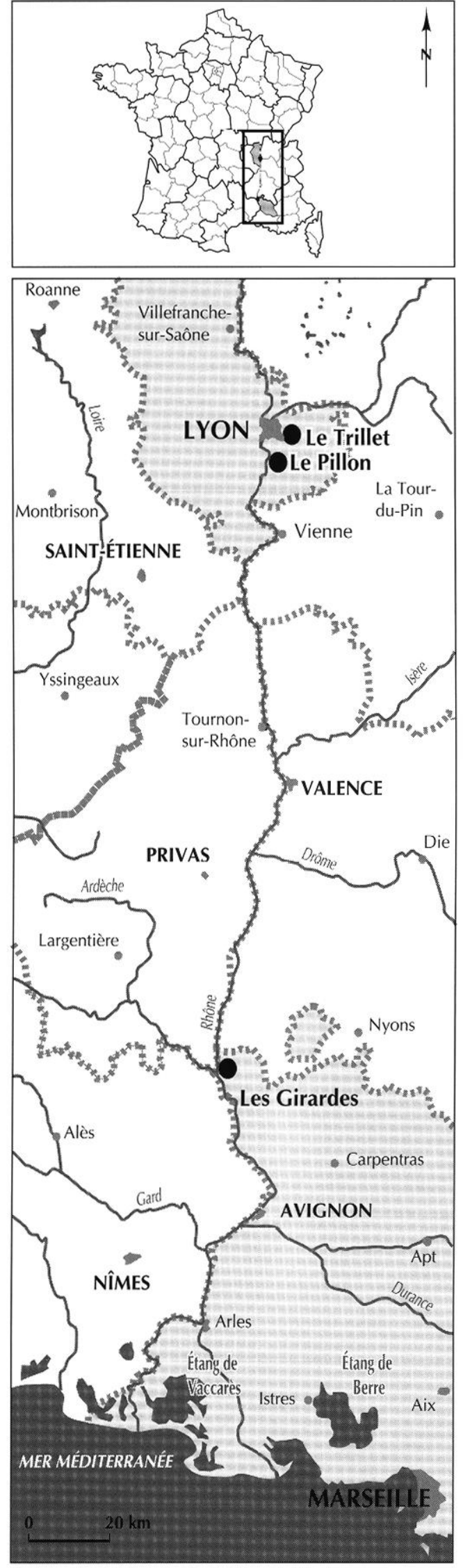

ristiques propres à ces petits ensembles afin d'en définir la nature: identité chronoculturelle, nature du groupe social. Nous tenterons de préciser leur datation et leur relation avec les structures domestiques, de souligner les traits particuliers de leurs pratiques funéraires (typologie des architectures en matière périssable, modalités de dépôt du mobilier, traitement des individus suivant l'âge et le sexe) et d'analyser les lois qui régissent leur « recrutement ». Enfin, les résultats seront discutés dans la problématique générale définie pour l'Antiquité tardive.

\section{PRÉSENTATION DES SITES}

\section{LE PILLON À MARENNES (RHÔNE)}

Le gisement du Pillon se situe à l'est de la commune de Saint-Symphorien-d'Ozon (Rhônc), à une vingtaine de kilomètres au nord de Vienne en Narbonnaisc (fig. 1). Dans ce secteur, la topographic présente deux grandes unités définies par les collines morainiques qui culminent à $362 \mathrm{~m} \mathrm{NGF}$ et la plaine à faible pente. Le site du Pillon est installé dans ce contexte, traversé par des cours d'eau, dont l'Ozon au sud et l'Inversé à l'est. L'établissement s'inscrit par son orientation dans la centuriation viennoise (Chouquer, Favory, 1980) et se place probablement dans les limites de la villa de Saint-Fréjus sur la commune de Marennes, en activité jusqu'à la fin de l'Antiquité tardive.

La fouille, menée par Serge Martin, s'est déroulée dans le cadre d'une opération préventive effectuée lors de la construction de l'autoroute A 46 sud pour le contournement de Lyon. Deux campagnes réalisćes en 1991 et 1992 ont permis de reconnaître la totalité des limites d'une ferme de type indigène à enclos fossoyés et un ensemble funéraire situé dans la partie septentrionale de l'emprise décapée (fig. 2). Le plan principal est constitué de fossés $(1,2,3,4,10,11,12)$ qui forment un quadrilatère irrégulier de $60 \mathrm{~m}$ sur $75 \mathrm{~m}$. L'intérieur de cet espace ainsi délimité est subdivisé par quatre fossés (5-6-7-605) qui forment cinq parcelles (A, B, C, D et E).

Fig. 1 - Situation géographique des gisements étudiés
dans la moyenne vallée du Rhône (DAO:G. Macabéo) 


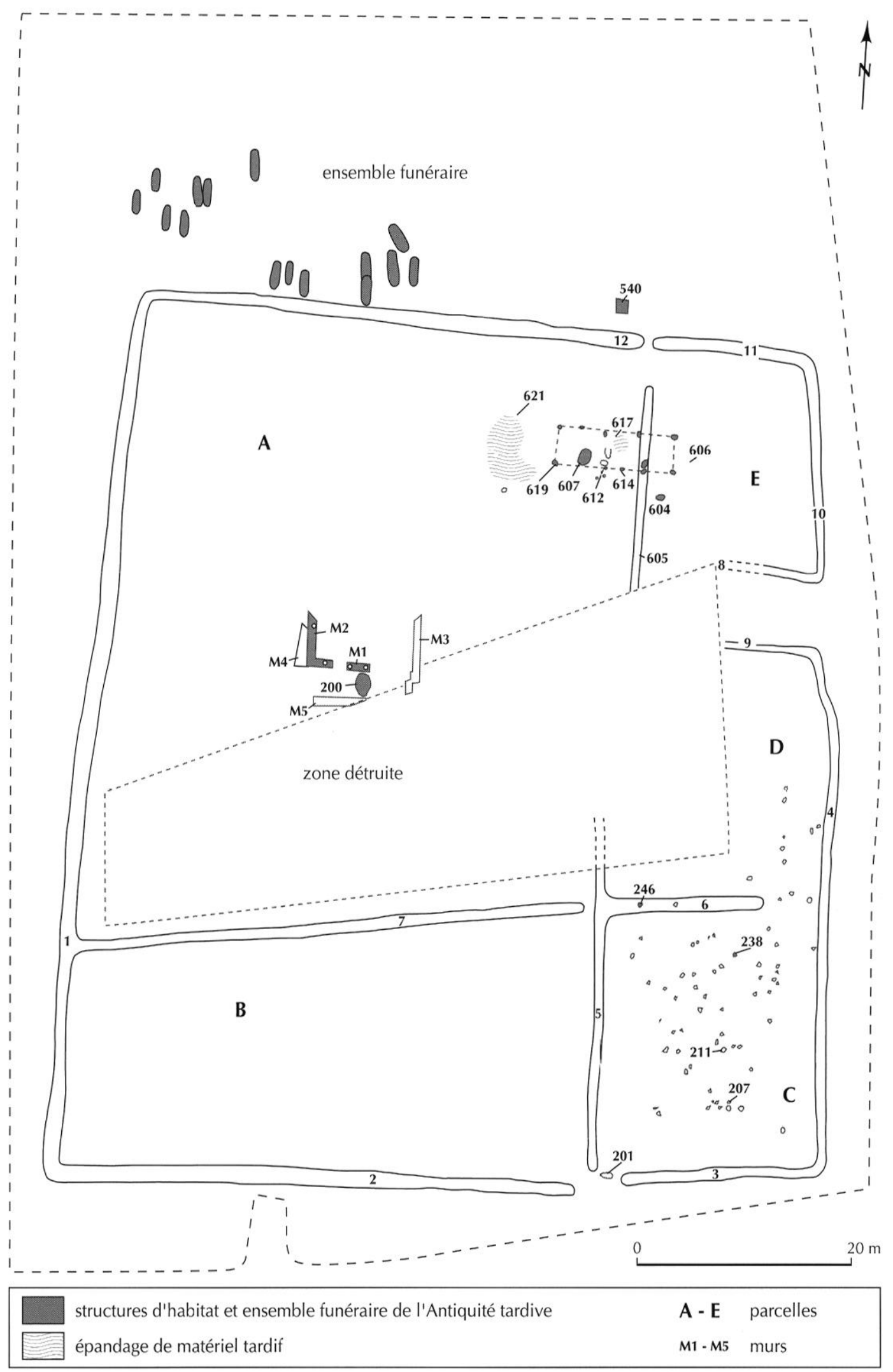

Fig. 2 - Plan général du Pillon à Marennes (relevé : S. Martin ; DAO : F. Blaizot). 


\section{L'HABITAT}

La circulation d'une parcelle à l'autre s'effectue par des interruptions d'une largeur de $1 \mathrm{~m}$ dans les fossés. À l'est, deux fossés (8 et 9 ) délimitent une entrée d'une largeur de $6 \mathrm{~m}$. Il s'agit d'un accès en cavée constitué de galets et de tuiles aménagé directement sur la terrasse morainique; par rapport au niveau d'apparition des structures, le niveau de circulation est situé en contrebas. Le fossé 605 , perpendiculaire à l'entrée, n'a livré aucun mobilier. Il est donc difficile de déterminer s'il correspond à une subdivision de l'espace à un moment où cette entrée était condamnée ou en service, ou s'il constitue à l'origine la limite est de la parcelle A ; dans ce cas, les parcelles $\mathrm{E}$ et $\mathrm{D}$ seraient des rajouts. À l'appui de la seconde hypothèse, on note que sur tout le site le seul mobilier attribuable au ${ }^{\mathrm{er}} \mathrm{s}$. a été recueilli dans les fossés 1,7 et 12 qui délimitent la parcelle A. L'enclos A ( $56 \mathrm{~m} \mathrm{x}$ $47 \mathrm{~m}$ ) est occupé par un bâtiment très arasé (M3, M4, M5) et détruit en partie par les travaux autoroutiers. Il se caractérise par un solin de tuiles et de galets. La date de construction de ce bâtiment est inconnue en l'absence de mobilier céramique. Seules son orientation et sa situation centrale dans la parcelle A semblent indiquer qu'il fait partie du système fossoyé originel. La parcelle $B$, qui jouxte la parcelle $\mathrm{A}$ au sud, ne présente aucun vestige, tandis que les parcelles $\mathrm{C}$ et $\mathrm{E}$ livrent du mobilier des II'-III'-IV $-V^{e}$ s. (cf. infra).

Le bâtiment de la parcelle $\mathrm{A}$ a fait l'objet d'une reconstruction marquée par deux murs (M1, M2) construits de galets à joint sec, auxquels sont associés, dans l'angle conservé, quelques blocs de schiste. Quatre trous de poteau de $50 \mathrm{~cm}$ de diamètre, repérés dans les murs, permettent d'envisager une élévation en matériaux légers. Le mur M2 a détruit la paroi est du mur M4 et est installé dans le comblement d'une dépression qui comporte des tessons de sigillée claire $B$. L'installation de ce nouvel édifice ne peut donc être antérieur aux $\mathrm{II}^{\mathrm{e}}-\mathrm{III} \mathrm{I}^{\mathrm{e}} \mathrm{s}$. Une monnaie de Magnence, trouvée dans la fosse US 200, témoigne d'une fréquentation de ce secteur au IV $\mathrm{I}$.

Directement au sud du mur M1, on relève la présence d'une fosse (200) qui renferme de nombreux charbons et nodules d'argile rubéfiée, des éléments de plomb fondu, ainsi qu'un grand nombre de tessons de céramiques à revêtement argileux apparentés à la sigillée claire $B$. L'état de conservation de ces céramiques ne permet pas de déterminer s'il s'agit uniquement de produits des $\mathrm{II}^{\mathrm{e}} \mathrm{III}{ }^{\mathrm{e}} \mathrm{s}$. ; en effet, quelques fragments pourraient être rapprochés des productions dites sigillées claire $\mathrm{B}$ tardives ou luisantes du $\mathrm{IV}^{\mathrm{e}} \mathrm{s}$. Ainsi, le bâtiment semble avoir fait l'objet d'une occupation tardive.

Les parcelles $\mathrm{C}$ et $\mathrm{D}$ ont fourni quarante-sept trous de poteau dont deux ont été pratiqués dans le comblement du fossé 6 . Seul le remplissage de quatre d'entre eux a livré de la céramique. Dans les structures 211 et 207 ont été recueillies des sigillées claire $\mathrm{B}$ produites aux II $^{\mathrm{e}}-\mathrm{III}^{\mathrm{c}} \mathrm{s}$. tandis que du matériel appartenant à un faciès plus tardif des $I V^{\mathrm{c}}-\mathrm{V}^{\mathrm{e}}$ s. comble les structures 246 et 238 . L'accès à l'enclos sud est marqué par des dalles posées à plat (201) parmi lesquelles se trouve de la céramique des II ${ }^{e}-$ III $^{e} s$. comprenant quelques éléments douteux pouvant appartenir au $I^{\mathrm{e}} \mathbf{s}$. La fonction de ces parcelles ne peut être déterminée en l'absence de témoins tangibles; cette " forêt de trous de poteau » désigne des installations en matériaux légers de petite taille, mais aucun plan n'apparaît clairement. On remarque cependant des alignements parallèles à l'axe des fossés. S'agit-il pour certains de palissades correspondant à une réorganisation de l'espace?

À cheval sur les parcelles A et $\mathrm{E}$ et sur le comblement du fossé 605 , se développent deux alignements de trous de poteau parallèles à l'axe du fossé 12. Ils permettent de restituer un bâtiment de forme rectangulaire, d'une longueur de 10,20 m et d'une largeur de 2,20 m, matérialisé au sol par neuf poteaux. Au nord, l'alignement orienté est-ouest est situé à une distance de $8 \mathrm{~m}$ du fossé 12. Cet édifice, d'une surface de $26,5 \mathrm{~m}^{2}$, possède une nef unique. Les poteaux porteurs des côtés latéraux ne sont pas répartis de manière régulière, malgré leur diamètre constant. Certains sont pourvus d'un blocage de plaques de schiste. On note l'absence de renfort des poteaux corniers. L'ensemble du matériel céramique issu des trous de poteau $612,619,614,617$ est caractéristique des $I V^{e}-V^{e} s$. avec une abondance de céramiques luisantes savoyardes. Deux foyers ont été identifiés (540 et 604); ils se situent à l'extérieur du bâtiment, le 540 se trouve même au nord à l'extérieur de l'enclos. Trois aménagements principaux, dont une fosse (607) et deux niveaux d'épandage de mobilier et de matériaux $(617,621)$, présentent exactement le même type de mobilier. Dans l'épandage 617 , on trouve deux monnaies datées de la fin du IV $\mathrm{V}^{\mathrm{c}}$ et du début du $\mathrm{V}^{\mathrm{e}} \mathrm{s}$. Par ailleurs, les tessons découverts dans le comblement du silo 607 appartien- 
nent à des céramiques luisantes savoyardes d'excellente qualité. Localisé dans la partie sud-ouest du bâtiment, ce silo a entaillé la terrasse. L'abandon qui s'est effectué en deux temps se caractérise par une série d'apports organiques mêlés à des rejets de foyer. Une cuvette située à l'est (606) renferme également des tessons attestant la datation tardive de cet ensemble.

\section{L'ENSEMBLE FUNÉRAIRE}

Il est composé de quinze sépultures ( $c f$. inventaire, p. 345-350) situées à l'extérieur de l'enclos principal, en périphérie du fossé 12 qui ferme le côté nord de l'établissement (fig. 2). Ainsi, bien que ce fossé ait été comblé à la fin du I ${ }^{\text {er }}$ s., l'enclos était toujours présent dans le paysage. Leur répartition définit trois groupes (fig. 3), l'un à l'ouest comporte sept sépultures, le deuxième à l'est en compte cinq, tandis qu'entre ces deux secteurs sont regroupées trois autres fosses. Toutes sont orientées sur l'axe nord-sud, perpendiculairement au fossé nord. Un décapage large réalisé autour de cette zone n'a révélé aucune autre tombe.

\section{Conclusion}

Hormis les fossés de la parcelle A et probablement le premier bâtiment, le site n'a livré aucun vestige attribuable au $\mathrm{I}^{\mathrm{er}} \mathrm{s}$; ; on note tout particulièrement l'absence de mobilier résiduel en dehors des fossés, ce qui laisse envisager une occupation de faible ampleur. L'installation de l'Antiquité tardive se caractérise par un réaménagement de l'édifice central et par la création d'un bâtiment sur poteaux. Ce dernier reprend l'orientation du fossé 12 comblé à la fin du Haut-Empire, mais recoupe le fossé 605 dont ni la fonction ni la date ne sont élucidées. Les vestiges de la parcelle D sont trop peu abondants pour interpréter sa fonction. L'organisation de l'ensemble indique une distribution fonctionnelle des espaces, la partie résidentielle (parcelles $\mathrm{A}$ et $\mathrm{E}$ ) étant éloignée de la parcelle $\mathrm{C}$ dont la vocation était peut-être différente. La parcelle $B$, dépourvue de structures, pourrait avoir servi à des fonctions agricoles, voire au pacage des animaux. Mis à part le fossé 605 , les fossés internes semblent exister en tant que limites aux $I V^{\mathrm{e}}-\mathrm{V}^{\mathrm{e}} \mathrm{s}$., puisque de nombreux alignements de trous de poteau situés dans les parcelles $\mathrm{C}$ et $\mathrm{D}$ reprennent leurs axes. De même, aucune

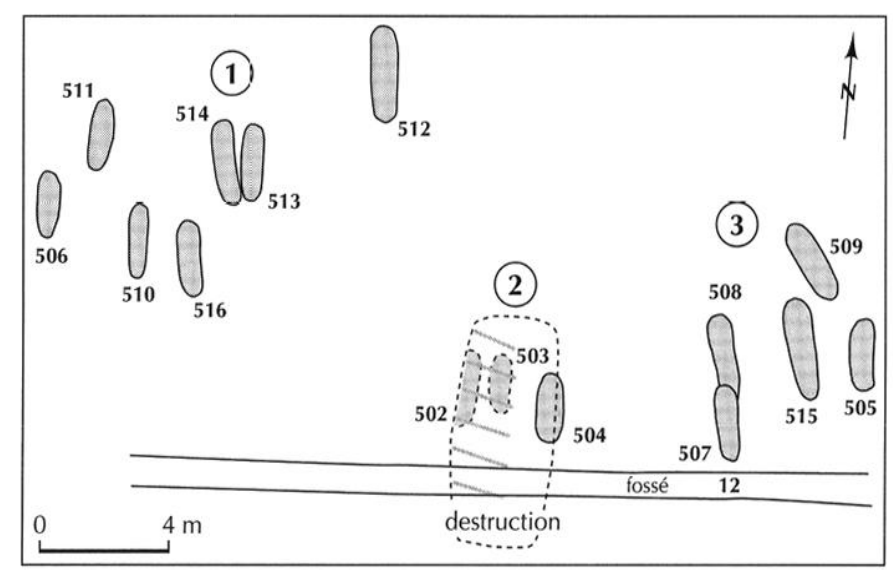

Fig. 3 - Plan de la nécropole du Pillon à Marennes :

1-3, groupes de tombes (relevé : S. Martin; DAO : F. Blaizot).

structure tardive n'empiète sur les fossés périphériques : le bâtiment sur poteaux respecte l'orientation des fossés nord et aucune sépulture ne recoupe ces derniers; il semblerait donc que l'enclos périphérique, dont certains fossés paraissent comblés à la fin $d u I^{\text {er }} s$., marquait encore d'une manière ou d'une autre l'une des limites de l'occupation du Bas-Empire. Nous n'avons cependant relevé aucun réaménagement des fossés à moins que les trous de poteau relevés dans le fossé 6 constituent les restes d'une palissade, mais l'importante érosion générale du site est peut-être en cause.

F. B., C. B. et S. M.

\section{LE TRILLET À MEYZIEU (RHÔNE)}

Le site du Trillet à Meyzieu a été fouillé dans le cadre de l'opération autoroutière A 42/A 43 (contournement de Lyon). Il se trouve en limite sud-ouest de la commune de Meyzieu et en partie sur celle de Décines-Charpieu, dans le Rhône (fig. 1). Il a été décapé sur une surface de $5000 \mathrm{~m}^{2}$ environ. Le terrain est constitué de moraine qui forme à cet endroit une butte assez marquée. Le site a livré les vestiges d'une occupation domestique du HautEmpire et du Bas-Empire et un ensemble funéraire de trente-deux sépultures à inhumation (fig. 4).

Les vestiges antiques les plus anciens sont attribués aux $\mathrm{I}^{\text {er }}$ et II ${ }^{\mathrm{e}} \mathrm{s}$. après J.-C. Ils se caractérisent par un réseau de fossés palissadés délimitant un enclos carré de 35,50 m de côté. Ce dernier renferme deux structures 


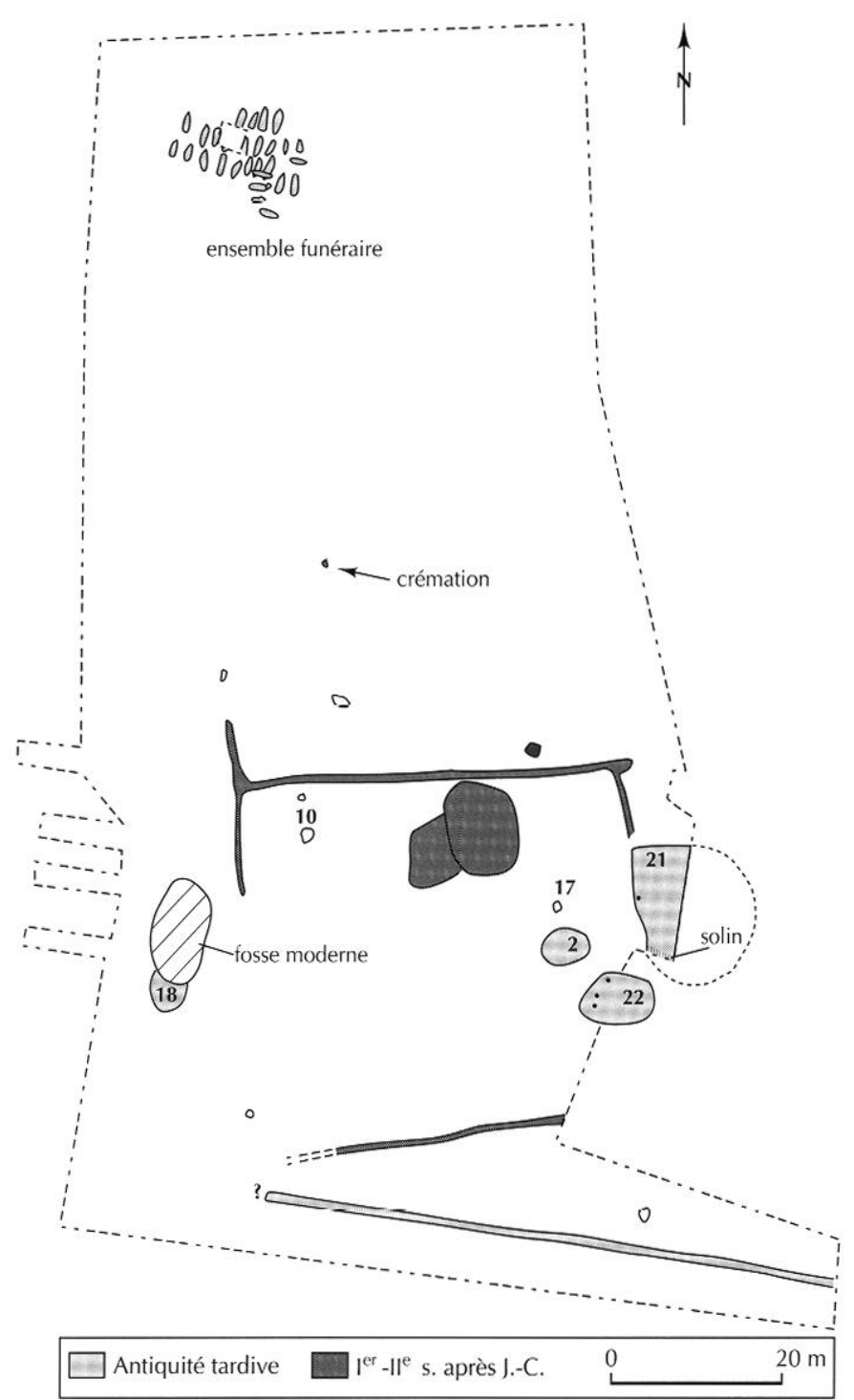

Fig. 4 - Plan général du Trillet à Meyzieu (relevé : D. Frascone; DAO : F. Blaizot).

d'habitat superposées. La première, qui possède un sol en terre battue et une banquette sur un côté, a été détruite par un incendie. La seconde, qui repose sur le niveau de destruction de la précédente, a connu deux occupations successives. Elle comportc un muret de galets qui la divise en deux pièces. Leur fonction n'a pas pu être déterminée. Une fosse contenant un dépôt de crémation en urne, daté du $\mathrm{II}^{\mathrm{c}} \mathrm{s}$., se trouve à environ $25 \mathrm{~m}$ au nord de l'enclos. Les quelques fosses et foyers, situés autour des deux structures, ne sont pas datés.

La deuxième occupation concerne l'Antiquité tardive, représentée par quatre structures d'habitat et un ensemble funéraire installé à $60 \mathrm{~m}$ au nord de l'enclos.

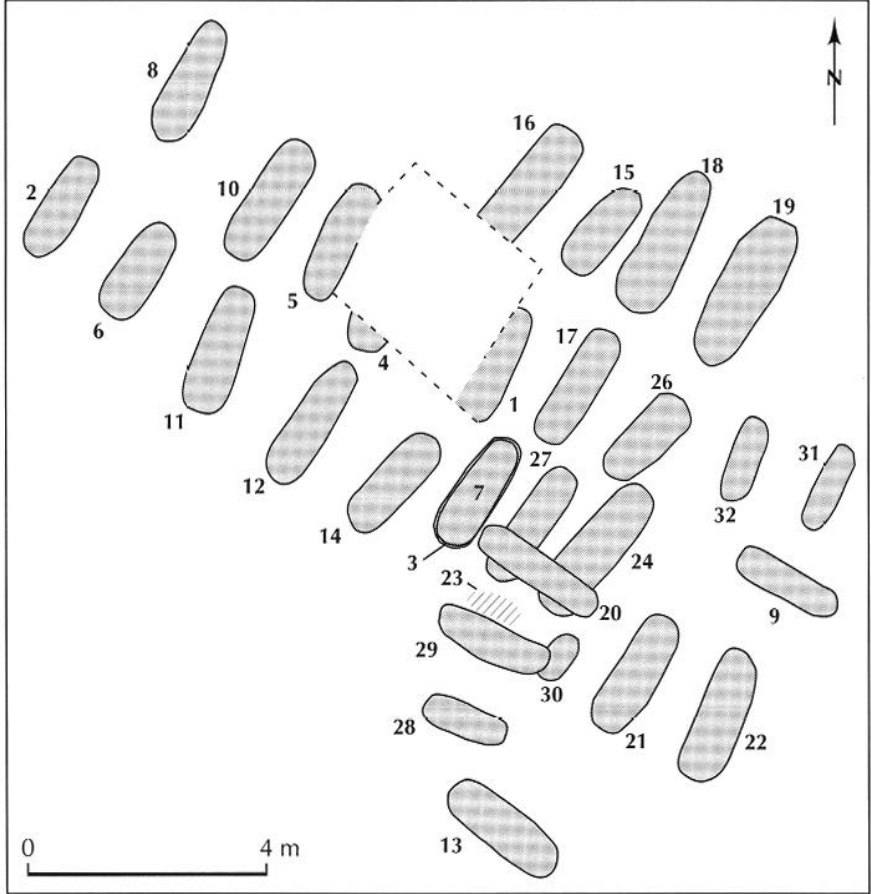

Fig. 5 - Plan de la nécropole du Trillet à Meyzieu (relevé : D. Frascone; DAO : F. Blaizot).

Les premières ne respectent pas l'enclos, puisque deux d'entre elles recoupent le fossé est : elles se développent sur l'axe est-ouest. L'une (18) cst en partie détruite par une fosse moderne. Ces structures, dont la taille varie de $12 \mathrm{~m}^{2}$ à $84 \mathrm{~m}^{2}$, présentent des aménagements variés. Elles sont définies comme des « structures excavées aménagées » (21-18-2) selon le terme retenu sur un site proche (Coquidé, Vermeulen, 1999, p. 239) et des greniers (22). L'une possède un sol de galets traversé par des trous de poteau (22), tandis que les autres sont excavées $(2,21,18)$; certaines sont ceintes d'un solin périphérique de galets qui supportait un mur en terre (21 et 22). La structure 21 , la plus grande $(12 \mathrm{~m} \times 7 \mathrm{~m})$, est divisée en deux par un solin de galets. Un aménagement interne est également supposé dans la structure 18 sur la base d'un amas de galets effondrés. Aucune ne comporte de foyer et il n'existe aucun élément pour leur associer les deux structures de combustion (10 et 17) trouvées à l'extérieur. La couche de destruction des " cabanes " 2, 18 et 21 a livré de nombreux clous de charpente et des tuiles, laissant envisager une toiture relativement élaborée. La présence d'outils (serpes, burins, crochets, couteaux) témoigne d'activités artisanales et agricoles, mais celle de vaisselle de table 
(141 fragments), de monnaies et d'objets de la vie quotidienne indique également une occupation domestique. Le matériel céramique des quatre structures appartient à l'Antiquité tardive mais une différence chronologique est perceptible entre les ensembles qui proviennent des " cabanes" 18 et 21 datées des $I V^{\mathrm{e}}-\mathrm{V}^{\mathrm{c}} \mathrm{s}$. et ceux des " cabanes » 2 et 22 s'apparentant plutôt aux III'-IV's. Les seules monnaies découvertes dans l'habitat sont issucs de la structure 21 ct sont antérieures au mobilier céramique puisqu'elles ont été frappées au cours du III's. (une monnaie de Galien 253-268, une monnaie de Philippe père 244-249, deux monnaies de Julia Domna et Élagabale de la première moitié du III ${ }^{e}$ s., un sesterce de Marc Aurèle). Les prospections engagées sur les parcelles attenantes n'ont livré aucun vestige, ce qui n'implique pas obligatoirement une faible extension du site du fait de la difficulté de repérer en prospection les gisements du Bas-Empire (Ouzoulias, Van Ossel, 2001, p. 157-162). On ne connaît pour l'instant aucune villa aux alentours. Ce type de structures est observé sur des sites proches, comme ceux de Chassieu-sous-Trillet et de Chassieu-l'Épine, entre le $\mathbf{I}^{\mathrm{er}} \mathbf{s}$. avant J.-C. et le III's. de notre ère (Coquidé, Vermeulen, 1999); il reste difficile d'interpréter précisément la fonction de ces différentes constructions et de définir les relations entre ces établissements.

Au sud de l'emprise se développe un fossé orienté d'est en ouest dont le comblement renferme du mobilier $d u V^{c} s$. qui pourrait être en relation avec les structures les plus tardives.

Les trente-deux sépultures forment un ensemble funéraire concentré sur une surface de $165 \mathrm{~m}^{2}$. Vingtquatre tombes ( $c f$. inventaire, p. 342-345), globalement agencées en trois rangées, présentent une orientation nord-sud, alors que dans la partie orientale, cinq ont une orientation est-ouest (fig. 5). Deux de ces dernières recoupent deux sépultures orientées nord-sud. Il n'existe aucun groupement de tombes au sein de l'ensemble funéraire.

\section{F., F. B. et C. B.}

\section{LES GIRARDES À LAPALUD (VAUCLUSE)}

Inscrit dans le cadastre B d'Orange au cœur de la plaine du Tricastin, le site des Girardes a fait l'objet de vastes décapages sur une zone d'emprunt du
TGV-Méditerranée, de 35 ha de superficie (fig. 1). Les fouilles, réalisées conjointement par Karine Roger et Philippe Boissinot (AFAN), ont permis non seulement l'exploration d'un établissement et de trois ensembles funéraires, mais aussi de plusieurs hectares de champs, des fossés et des chemins, donnant ainsi une image précise de l'organisation interne et de l'évolution d'un domaine antique (fig. 6).

En dehors d'une occupation protohistorique ponctuelle, le noyau de peuplement observé dans la partie occidentale de l'emprunt est daté du début de notre ère. Des constructions en bois et des silos matérialisent probablement cette première exploitation. Dès le courant du I Ir s. après J.-C., un bâtiment en pierre destiné à la vinification est construit (A); le peu de place disponible pour une éventuelle pars urbana laisse supposer que la maison du maitre était ailleurs, probablement dans l'agglomération proche de Saint-Paul-Trois-Châteaux. Parallèlement à ces activités agricoles ou légèrement plus tard, une activité proto-industrielle, liée à la métallurgie, est mise en place dans un deuxième bâtiment (B). On connaît, pour cette période, deux petits groupes de sépultures constituées de dépôts de crémation en fosses. L'un au nord de l'emprunt (1), le second au sud (2), ils sont tous deux établis à l'orée des champs. Dans ces derniers, la culture de la vigne est alors dominante, même si l'on doit associer une culture céréalière dès le commencement de l'exploitation.

À la fin du $\mathrm{II}^{\mathrm{e}} \mathrm{s}$., les études environnementales mettent en évidence une réelle transformation du paysage : le climat est caractérisé par la recrudescence de violents orages et un début de reconquête forestière est attesté.

$\mathrm{Au}$ début du $\mathrm{III}^{\mathrm{e}}$ s., d'importantes modifications sont observées dans le bâtiment principal qui ne sert désormais plus qu'à la fabrication du vin, comme cela a déjà été mis en évidence sur plusieurs sites contemporains. Un nouvel édifice, au sud de l'emprunt, servant probablement d'étable $(\mathrm{C})$, vient s'ajouter aux constructions restructurées. Au cours de ce siècle, l'essentiel du vignoble est certainement abandonné, la culture des céréales reprend alors de l'ampleur.

À la transition $\mathrm{III}^{e}-\mathrm{IV}^{\mathrm{e}} \mathrm{s}$., un ensemble funéraire à inhumation est installé au détriment des vignobles abandonnés (3). À l'instar d'autres aménagements dans le secteur de l'habitat, ces pratiques indiquent une faible occupation des lieux jusque dans le courant du IV ${ }^{e} s$. 


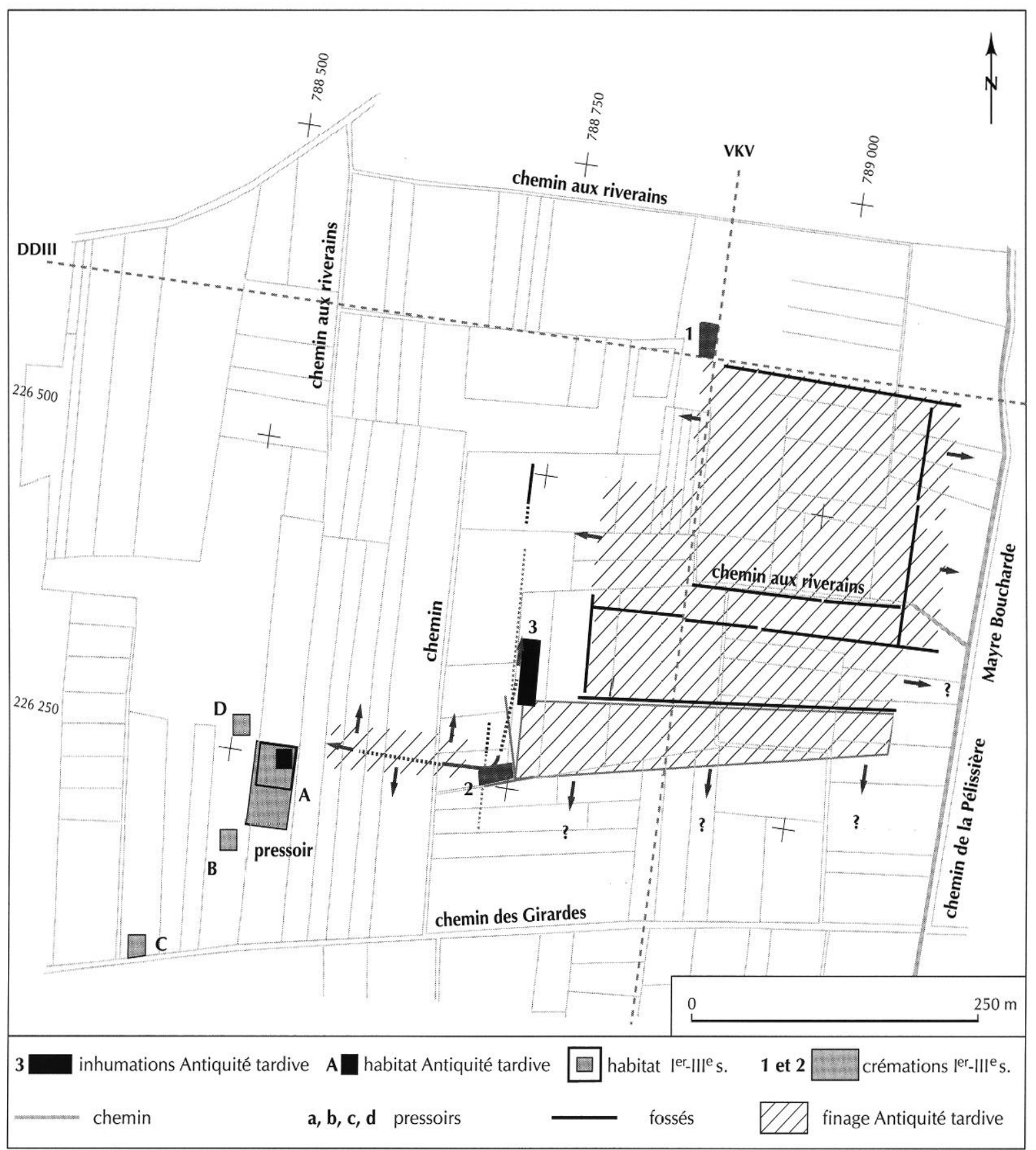

Fig. 6 - Plan général des Girardes à Lapalud (relevé : K. Roger et S. Lainé ; DAO : G. Macabéo).

Durant le début du haut Moyen Âge, une occupation ponctuelle est signalée par les prospections et matérialisée par deux foyers. Il s'agit des derniers indices de fréquentation du site avant la période moderne.

L'ensemble funéraire à inhumation de l'Antiquité tardive comprend dix-sept sépultures dont deux sont celles de canidés ( $c f$. inventaire, p. 350-353), constituant globalement deux groupes, l'un au nord et l'autre au sud (fig. 7). Il se situe à environ $500-600 \mathrm{~m}$ au nord-est de l'habitat proprement dit. À l'est, il est bordé de trois rangées de fosses de plantations de vigne (71), limite transgressée par le groupe de sépultures au sud. Une sépulture (SP5067) coupe un fossé de drainage orienté nord-est/sud-ouest (SP5020). Celui-ci est 


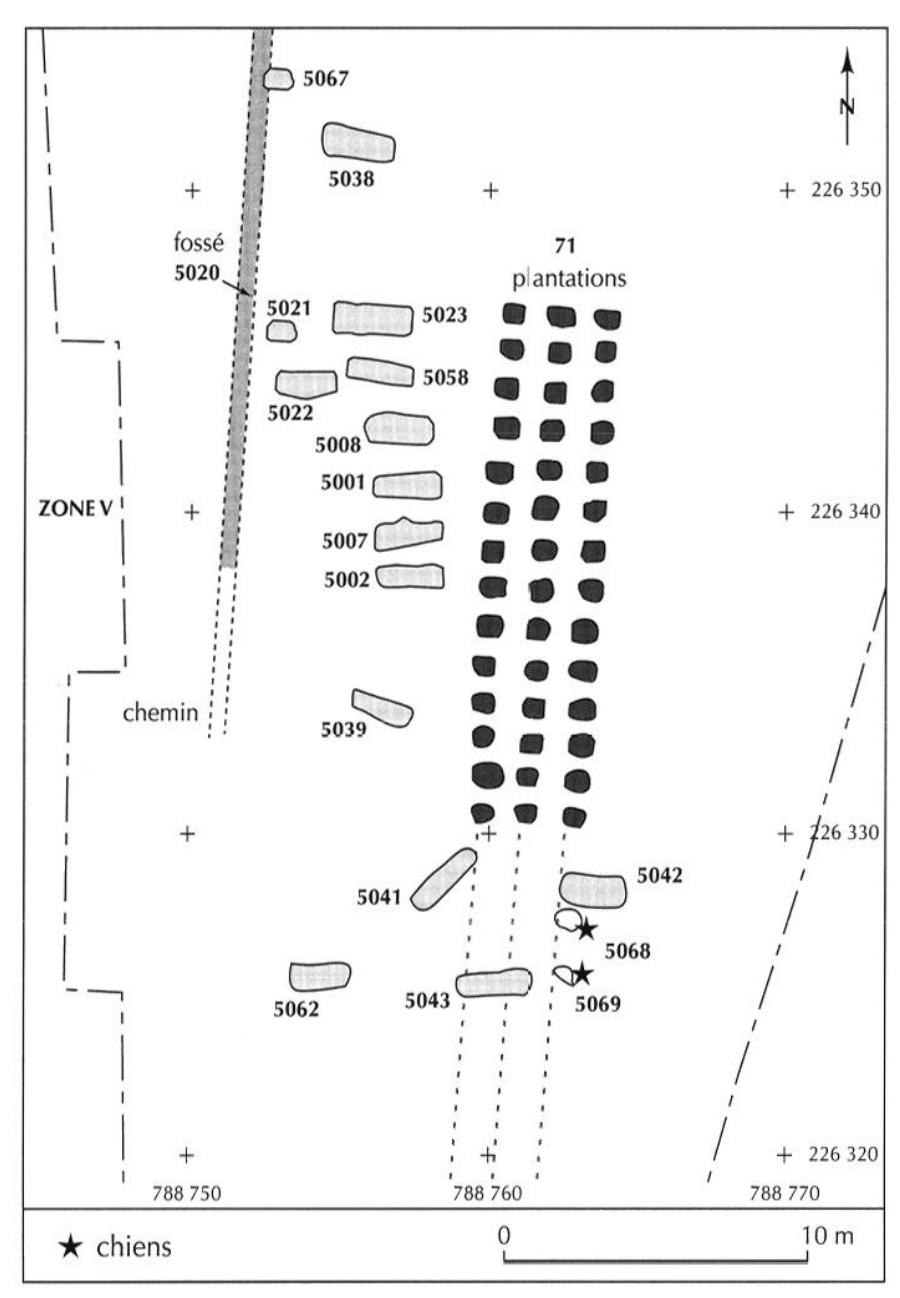

Fig. 7 - Plan de la nécropole des Girardes à Lapalud (relevé : K. Roger et S. Lainé ; DAO : G. Macabéo).

antérieur à un chemin dont la dernière utilisation est datée de l'Antiquité tardive (fig. 6). Dans son prolongement théorique, ce chemin passerait quelques mètres à l'ouest des sépultures. Toutes les fosses sont orientées est-ouest.

K. R.

\section{LES MODES D'INHUMATION}

\section{TYPOLOGIE ANALYTIQUE DES SÉPULTURES}

La qualité des vestiges et de la documentation est variable suivant les sites et de nombreuses sépultures n'ont livré aucune information nécessaire à l'identification du mode d'inhumation. Les données issues des
Girardes à Lapalud sont particulièrement indigentes, les ossements étant en très mauvais état de conservation (deux sépultures exploitables sur quinze) et la moitié des squelettes du Pillon à Marennes fournissent des données insuffisantes. Les sépultures du Trillet à Meyzieu ont été analysées à partir des clichés photographiques.

La position respective des ossements est décrite par rapport à la position anatomique de référence dans laquelle le squelette est placé debout, les membres en extension parallèles à l'axe longitudinal du corps. La nomenclature définit un système de plans horizontaux (perpendiculaires à l'axe longitudinal du corps) et deux systèmes de plans verticaux: les plans sagittaux (dirigés d'avant en arrière dont le plan médian dit plan de symétrie est un cas particulier) et, perpendiculaires aux précédents, les plans frontaux (droite ou gauche). Le plan médian distingue un versant droit et un versant gauche; les plans sagittaux, autres que le plan médian, définissent un versant latéral (le plus éloigné du plan médian) et un versant médial (qui regarde vers le plan médian). Par rapport aux plans frontaux, il existe un versant antérieur et un versant postérieur. Par rapport aux plans horizontaux, la terminologie anatomique distingue les versants supérieur (au-dessus) et inférieur (au-dessous), ce qui entraîne une confusion avec la terminologie habituelle en archéologie qui, elle, se rapporte à l'horizontale dans l'espace et non pas à un sujet en position anatomique. C'est pour cela que nous remplacerons les termes « au-dessus » et " au-dessous » par « en amont » et «en aval », l'extrémité amont du corps étant la tête et l'aval correspondant aux pieds.

\section{LES CERCUEIIS CLOUÉS}

Ce type de tombe concerne deux sépultures au Pillon, une aux Girardes et aucune au Trillet ( $c f$. inventaire, p. 348 et 352 ).

$\mathrm{Au}$ Pillon, les clous qui sont disposés latéralement au squelette reposent sur le fond de la fosse ; ils sont placés en vis-à-vis et paraissent avoir assemblé les planches en trois points : le premier au niveau des épaules ou des bras du corps, le deuxième à hauteur des cuisses ou des hanches et le dernier de part et d'autre des jambes et des pieds (fig. 8 et 9). Un clou supplémentaire est présent dans la sépulture SP509 (fig. 8), latéralement au pied gauche du squelette. Ceux situés en aval des pieds et en 
amont du crâne ne reposent pas sur le fond de la fosse, mais à plus de $10 \mathrm{~cm}$ au-dessus. Il est donc possible qu'à l'origine ces derniers aient servi non pas à assembler les

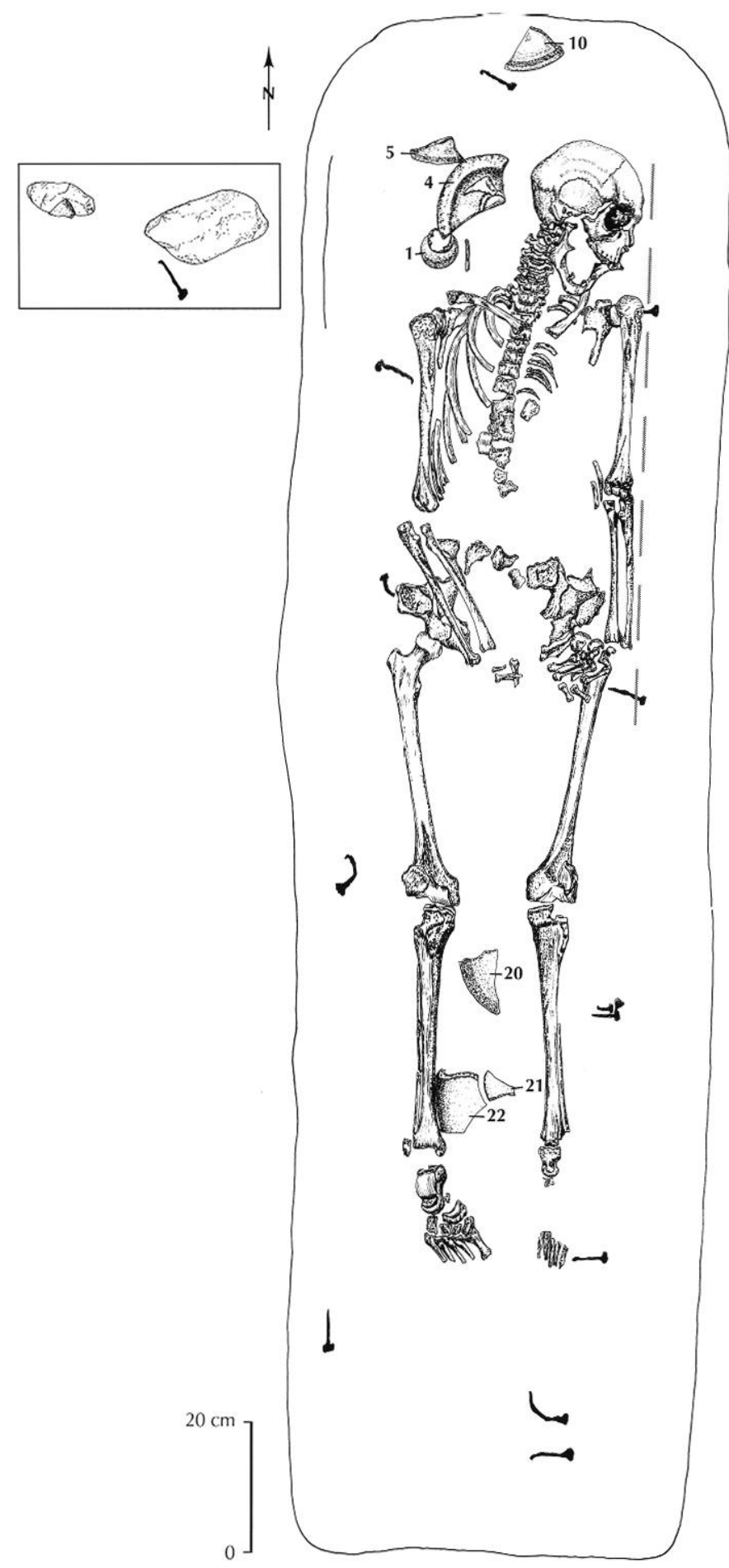

Fig. 8 - Le Pillon à Marennes, sépulture SP509: 1, gobelet ; les fragments $n^{o s} 10,4,5,20,21$ et 22 appartiennenl au vase $n^{\circ} 2$ (cf. pl. III). En pointillé : effet de délimitation (relevé et dessin : F. Blaizot ; DAO : G. Macabéo). éléments de la cuve mais à fixer le couvercle. On note que dans la sépulture SP509 les clous se trouvent à distance des pieds du squelette, ce qui indique que lc cercueil était long.

Une sépulture du Trillet comporte un seul clou (SP12, non illustrée) situé latéralement à la cheville gauche. Le fait qu'il soit unique indiquerait qu'il s'agit d'un élément de fixation ponctuel. Sa position, $10 \mathrm{~cm}$ au-dessus du fond de fosse, permet d'envisager qu'il contribuait à l'assemblage du couvercle d'un contenant monté sans clou à l'origine, celui-ci étant déterminé par des effets de contrainte enregistrés sur le squelette.

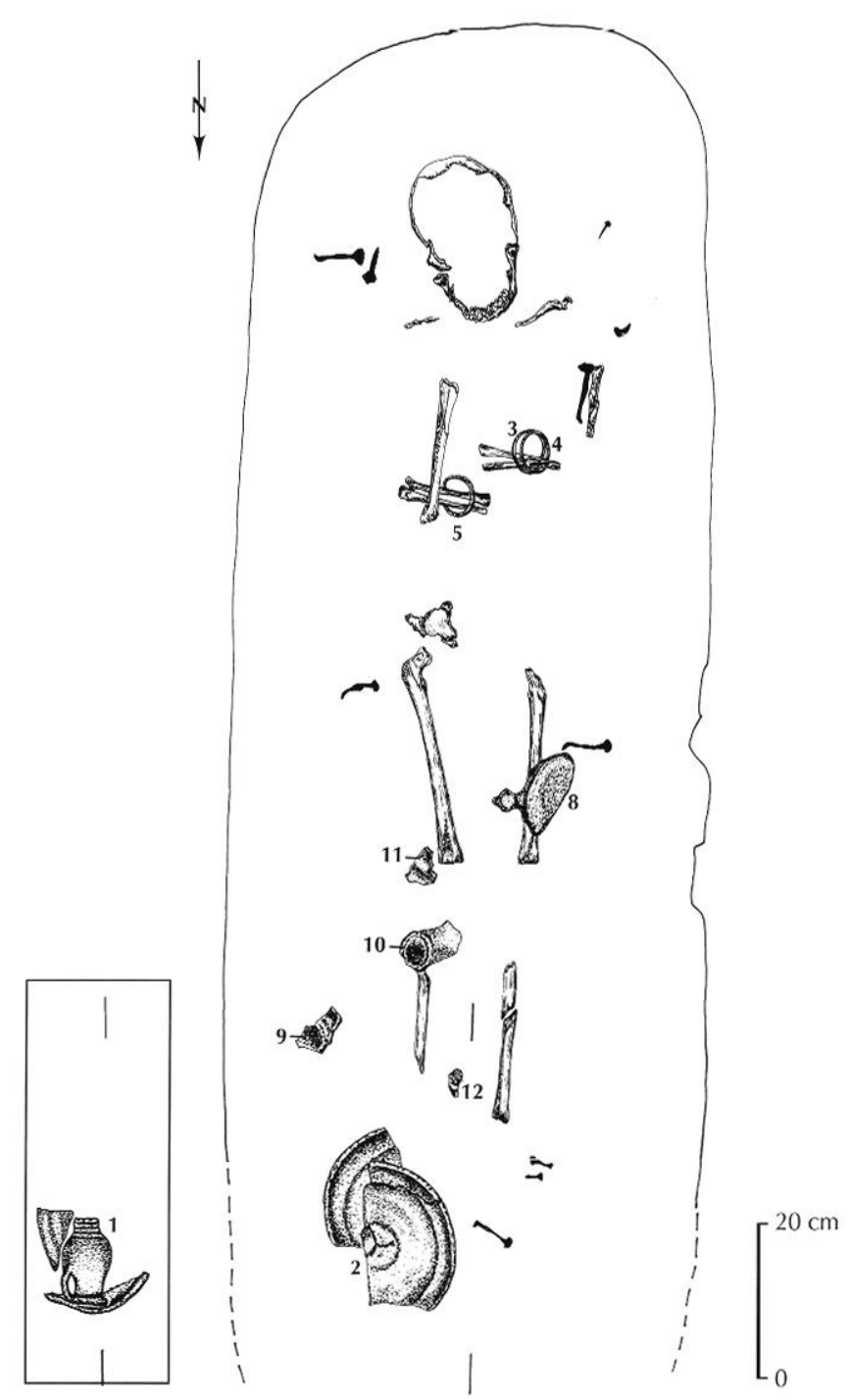

Fig. 9 - Le Pillon à Marennes, sépulture SP507. Les fragments de céramique $n^{* s}$ 9, 10, 11 et 12 appartiennent à la sépullure SP508 (cf. pl. III) (relevé et dessin : F. Blaizot ; DAO : G. Macabéo). 
Aux Girardes, les clous présentent la même disposition qu'au Pillon et l'un d'entre eux, retrouvé à une extrémité de la fosse, repose moins profondément que les autres, suggérant son appartenance au couvercle.

L'examen des squelettes in situ indique que les cercueils étaient de forme rectangulaire et relativement ajustés au corps en largeur. À l'appui de cette remarque, on relève des effets de contrainte latérale qui se traduisent principalement par le décalage en hautcur d'une épaule par rapport à l'autre, par des effets de délimitation et par l'absence d'effondrement latéral des pieds (fig. 8). Dans la sépulture SP507 du Pillon, le déplacement de l'humérus droit en direction médiale ne peut être imputé à la rupture d'un effet de contrainte - bras droit en appui contrc la paroi du ccrcucil - puisque la clavicule homolatérale est horizontale (fig. 9) ; son remaniement a probablement été causé par l'effondrement du contenant. La mauvaise conservation des os de la sépulture SP5041 des Girardes ne permet pas de préciser la taille du contenant.

Dans la moitié sud de la Gaule, le cercueil cloué connaît son apogée au IV ${ }^{\mathrm{e}}$ s., puis se raréfie et disparaît dans le courant du siècle suivant (Colardelle et al., 1996, p. 273), alors qu'il est utilisé jusqu'au VI ${ }^{\mathrm{c}}$ s. dans les régions du nord (Fréville, Journa, 1994). Par référence aux nombreuses nécropoles fouillées, les cercueils cloués du Pillon et des Girardes peuvent être datés sans ambiguité du Bas-Empire, ce qui est implicite compte tenu de la nature du mobilier ( $c f$. infra). La typologie des clous confirme en tout cas cette appartenance à l'Antiquité tardive, puisqu'ils se distinguent, par leur taille et leur morphologie, de ceux qui sont utilisés au bas Moyen Âge et à l'époque moderne. En effet, si tous ont des tiges de section carrée ces derniers se caractérisent par des têtes plates (Prigent, Hunot, 1996, p. 75), tandis que ceux des cercueils examinés ici possèdent des têtes circulaires convexes, typiques des clous rencontrés au Bas-Empire (Ratel, 1977, p. 84). La différence se situe également entre le nombre et l'emplacement des clous utilisés. Dans l'Antiquité tardive ils sont fixés par paires opposées au niveau des deux tiers distaux et du tiers médian du cercueil, alors que dans les périodes plus récentes ils apparaissent en grand nombre et sont situés sur tout le pourtour du contenant. La fixation du couvercle à l'aide de clous n'est pas systématique dans le Bas-Empire mais, lorsque c'est le cas, ils se trouvent placés aux extrémités (Ratel, 1977, p. 87).

\section{LES CONTENANTS NON CLOUÉS}

Ce terme est utilisé ici pour désigner un contenant dont les parois de bois ne sont pas assemblées à l'aide de clous, puisqu'il n'est pas toujours possible de distinguer, à travers les vestiges archéologiques, un coffre chevillé d'un coffrage fabriqué dans la fosse. Ce type d'architecture se rapporte à la quasi-totalité des sépultures interprétées du Trillet sauf quatre (SP2, SP3, SP5, SP9), à six sépultures du Pillon et, autant que l'état des ossements permette d'en juger, à au moins une sépulture des Girardes ( $c f$. inventaire, p. 352).

Dans ces exemples, l'analyse des anomalies taphonomiques du squelette reste le seul moyen de déterminer la présence initiale d'un contenant en matière périssable. La restitution est fondée sur la démonstration d'une décomposition en espace vide et sur la reconnaissance d'effets de contrainte ou de délimitation sur les os, observés à distance des parois de la fosse. Dans certains cas, la morphologie du contenant, voire ses techniques de construction (présence ou non d'un fond par exemple) ou d'installation dans la fosse (présence de cales inférieures) peuvent être précisées par l'étude des remaniements qui se sont produits sur les squelettes. L'absence de fond construit et la présence de calages inférieurs contribuent parfois à différencier un coffrage de planches d'un coffre chevillé.

\section{Le Trillet}

$\mathrm{Au}$ Trillet, on distingue trois formes de contenant, l'une rectangulaire, la deuxième trapézoïdale et la dernière hexagonale ( $c f$. inventaire, p. 342-345). De même, deux types de construction sont reconnus: le premier s'apparente à un coffrage de planches entre lesquelles le corps repose à même le sol et le second possède un plancher. C'est ainsi que la large classe des " contenants non cloués" est subdivisée en plusieurs types. Il n'existe pas de coincidence entre la forme rectangulaire ou trapézoïdale et la présence ou non d'un plancher.

Dans la série du Trillet, des précisions sur l'assemblage des planchers ont pu être apportées à deux reprises (sépultures SP16 et SP18). Sur le squelette de la sépulture SP16 l'extrémité latérale de la clavicule et un métacarpien situé en aval et à droite de la symphyse pubienne sortent du volume initial du cadavre (fig. 10). De même 
le tarse droit, qui s'est effondré latéralement puisqu'il apparaît par sa face médiale alors que le membre inférieur droit est en vue antérieure. Ces remaniements témoignent d'une décomposition en espace vide. On remarque une surélévation centrale de la colonne vertébrale, accompagnée d'une ouverture complète et particulière des côtes. Ce phénomène permet d'envisager que le plancher du coffre était constitué de deux planches, dont les parties latérales se sont affaissées. Dans cette sépulture, le redressement du crâne implique que le

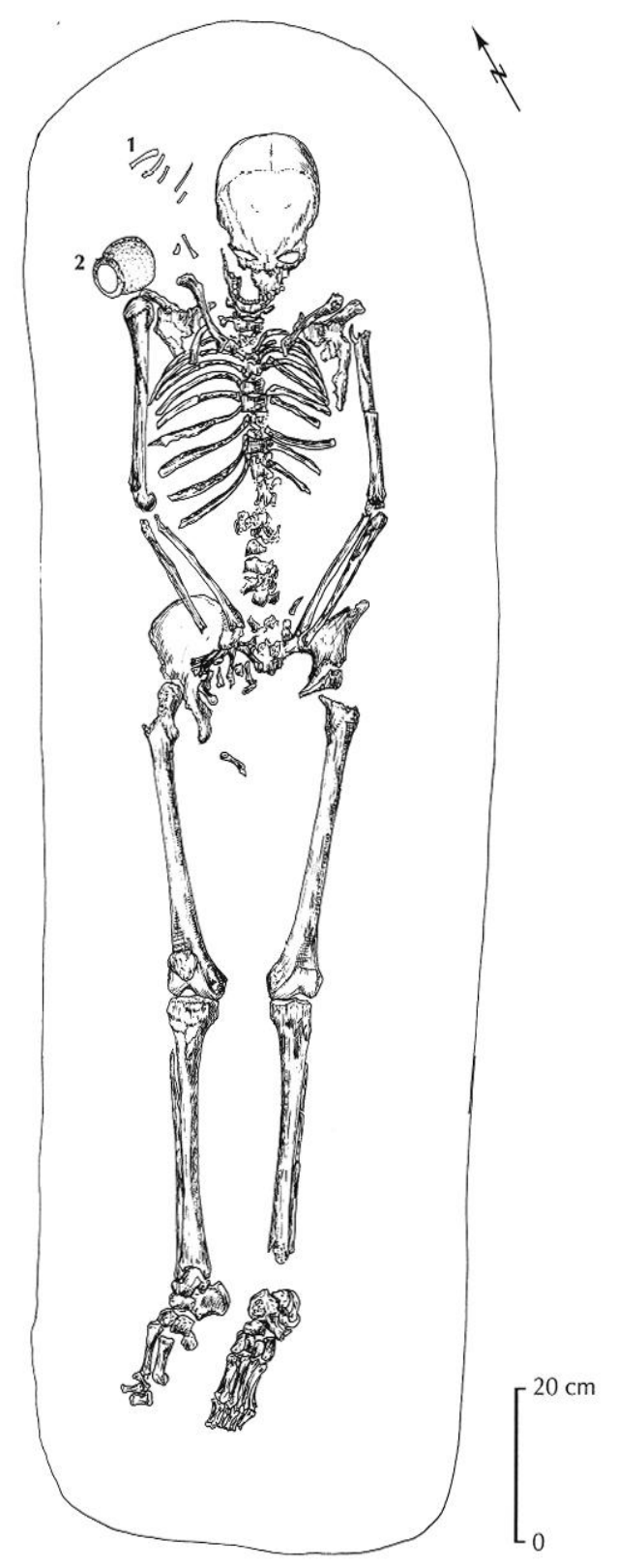

Fig. 10 - Le Trillet à Meyzieu, sépulture SP16 (cf. pl. V) (dessin: J. Cathalaa et F. Blaizot; DAO: G. Macabéo). contenant était court, ou en tout cas que le corps s'appuyait contre sa paroi transversale au nord.

Dans la sépulture SP6 (fig. 11), les vertèbres thoraciques qui se situent latéralement au coxal droit sortent du volume initial du cadavre et rendent compte d'une décomposition en espace vide. Par ailleurs, l'effet de délimitation observé sur tout le côté gauche du squelette à distance de la paroi de la fosse témoigne de la présence d'une planche à l'origine. On remarque que l'humérus droit est encastré entre deux pierres et l'hypothèse d'un fond en bois peut être retenue, ce phénomène traduisant un pourrissement des planches placées en appui sur ces pierres. La présence initiale d'un fond construit étant ainsi probable, la constriction de la moitié supérieure du thorax ne peut pas être attribuée à un effet de gouttière,

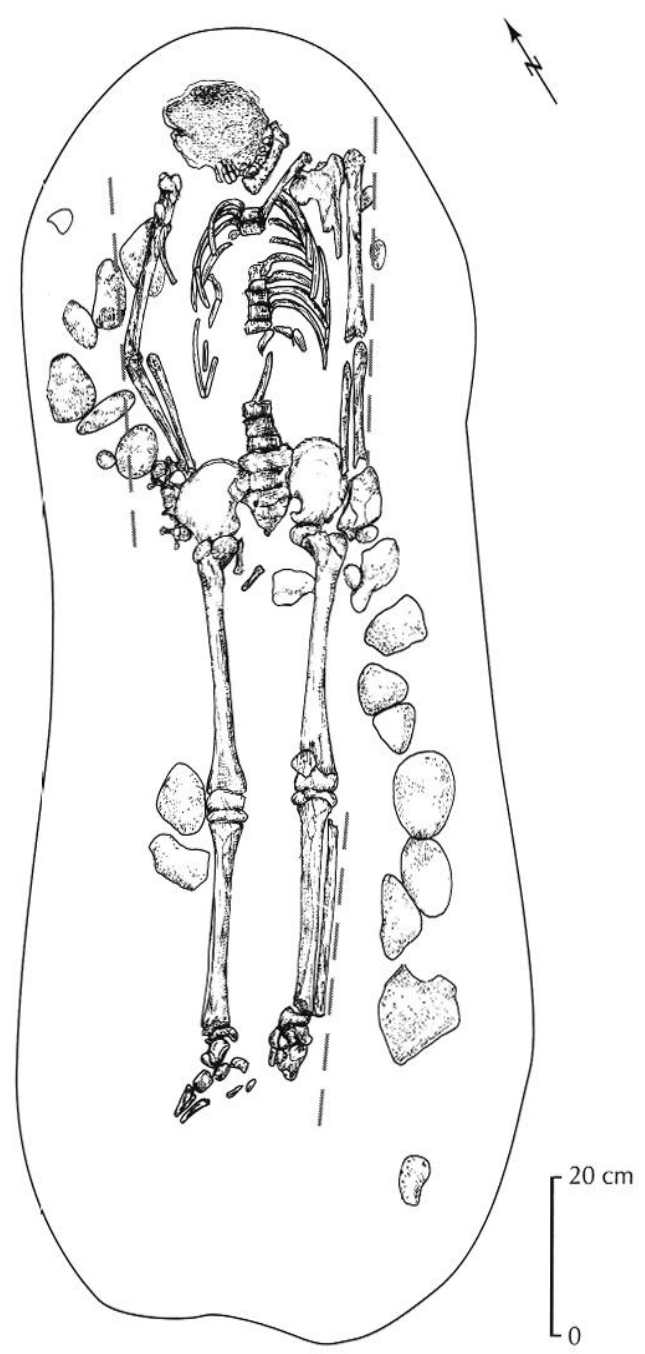

Fig. 11 - Le Trillet à Meyzieu, sépulture SP6. En pointillé : effet de délimitation (dessin: J. Cathalaa et F. Blaizot; DAO: G. Macabéo). 
mais à un certain effet de tassement du côté gauche, expliquant l'obliquité de la clavicule.

Des effets de contraintes latérales sont observés également sur les pieds. Ils indiquent parfois que le réceptacle était d'une largeur moins importante à ce niveau que dans la moitié supérieure du corps (forme trapézoïdale). C'est le cas du squelette de la sépulture SP27 (fig. 12), dont les coudes dirigés vers l'extérieur ont une emprise en largeur supérieure à ccllc des pieds. Malgré l'orientation médiale de l'extrémité de l'humérus gauche, on ne peut pas envisager un contenant de forme hexagonale en l'absence d'effet de

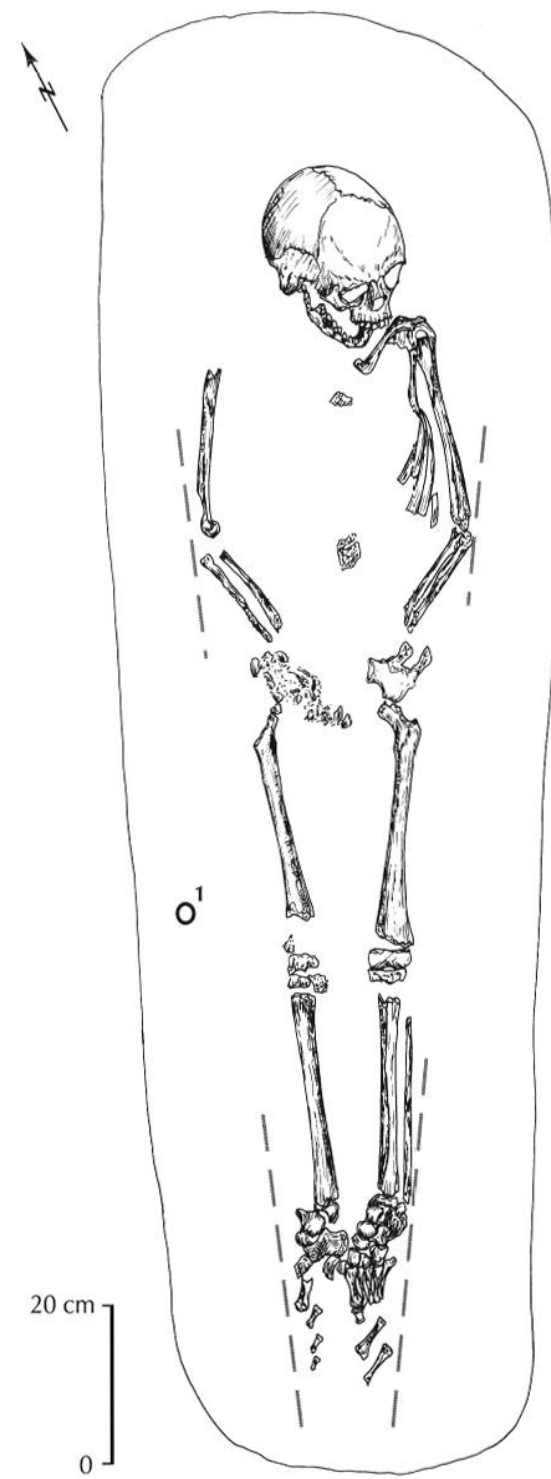

Fig. 12 - Le Trillet à Meyzieu, sépulture SP27 (cf. pl. V). En pointillé : contraintes latérales délimitant le contenant (dessin : J. Cathalaa et F. Blaizot ; DAO : G. Macabéo). contrainte relevé sur la scapula (en vue antérieure). La décomposition en espace vide est déduite de la dislocation du pied droit, dont deux métatarsiens sortent du volume initial du corps, tandis que la présence d'un contenant est argumentée par l'effet de contrainte enregistré à distance des bords de la fosse du pied gauche. Cette sépulture illustre également un exemple où le corps repose directement sur le sol. En effet, les cotes de profondeur enregistrées sur les humérus montrent qu'ils se situent $5 \mathrm{~cm}$ moins profondément que les vertèbres, ce qui est également le cas des pieds; la morphologie du fond de la fosse est donc incompatible avec l'hypothèse d'un plancher dans la mesure où le squelette ne présente aucune dislocation violente attestant un effondrement dans une " gouttière ".

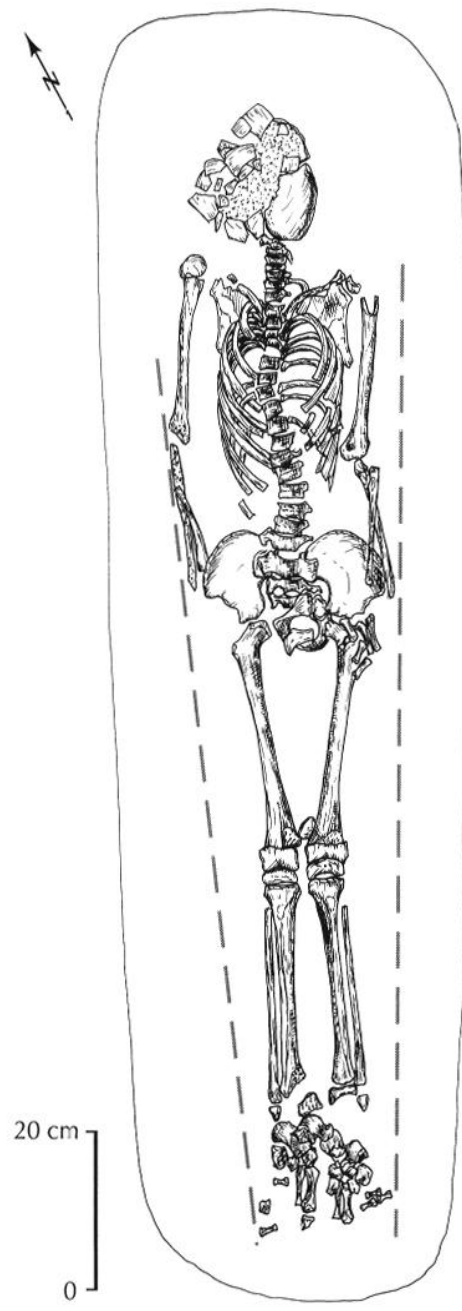

Fig. 13 - Le Trillet à Meyzieu, sépulture SP31. En pointillé : contraintes latérales, délimitation donnant la forme du contenant (dessin: J. Cathalaa et F. Blaizot ; DAO: G. Macabéo). 
L'examen du squelette de la sépulture SP31 montre que le corps a été déposé dans un contenant de forme trapézoïdale, dont la largeur au niveau des pieds est inférieure à celle qui prévaut dans la moitié supérieure du corps (fig. 13). Les effets de délimitation et de contrainte latérale, observés à distance des parois de la fosse, sont nets sur les pieds et sur les membres supérieurs (humérus droit décalé en hauteur, avant-bras gauche en équilibre instable), ce qui démontre bien l'existence d'un contenant à l'origine. Dans ce cas précis, le contenant possédait un fond, puisque l'ensemble du squelette témoigne d'un « effet de sol " par la segmenta-

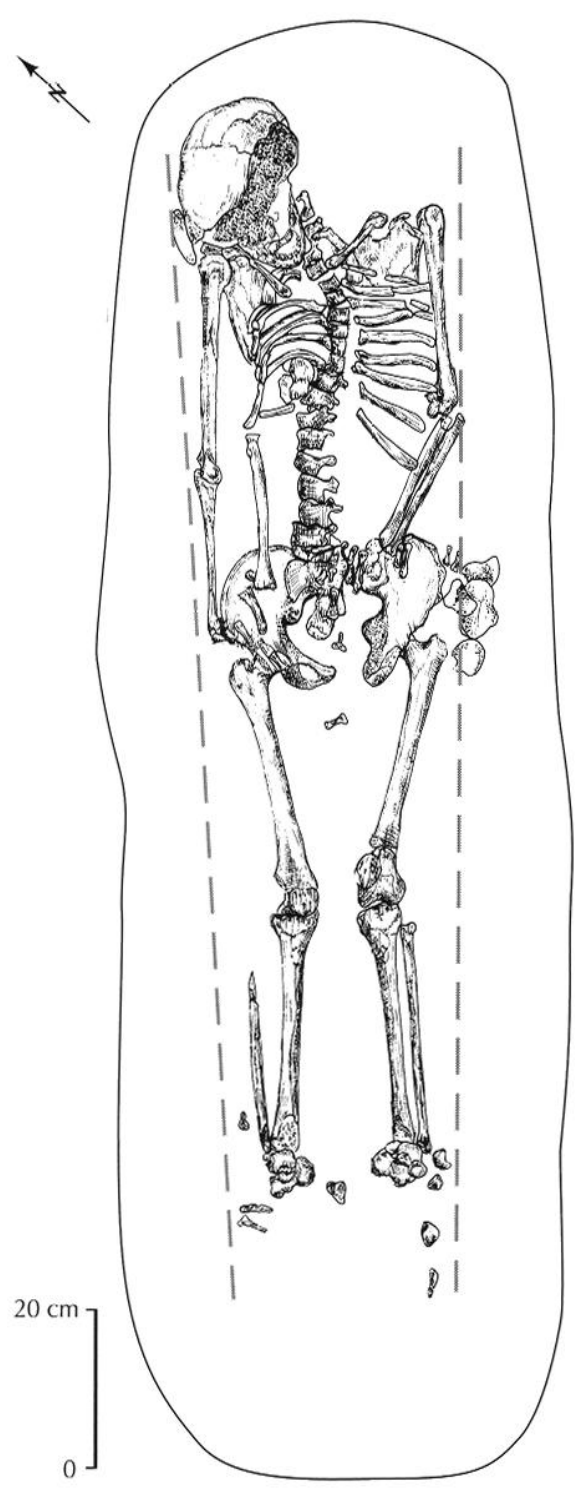

Fig. 14 - Ie Trillet à Meyzieu, sépullure SP22. En pointillé : contraintes latérales et délimitation permellant d'estimer la forme du contenant (dessin : J. Calhalaa et F. Blaizot ; DAO: G. Macabéo). tion et la sinuosité des vertèbres, et surtout par la remontée de la tête des côtes.

Le squelette de la sépulture SP22 illustre un dépôt pratiqué dans un contenant de forme rectangulaire (fig. 14). L'espace vide est démontré par le glissement du crâne vers l'avant et la droite, par le déplacement de deux phalanges de la main, l'une entre les cuisses et l'autre latéralement au coxal gauche et par la dispersion des os des pieds. Le contenant est déterminé par le décalage en hauteur de l'épaule gauche et par l'effet de délimitation qui affecte les métatarsiens droits, orientés

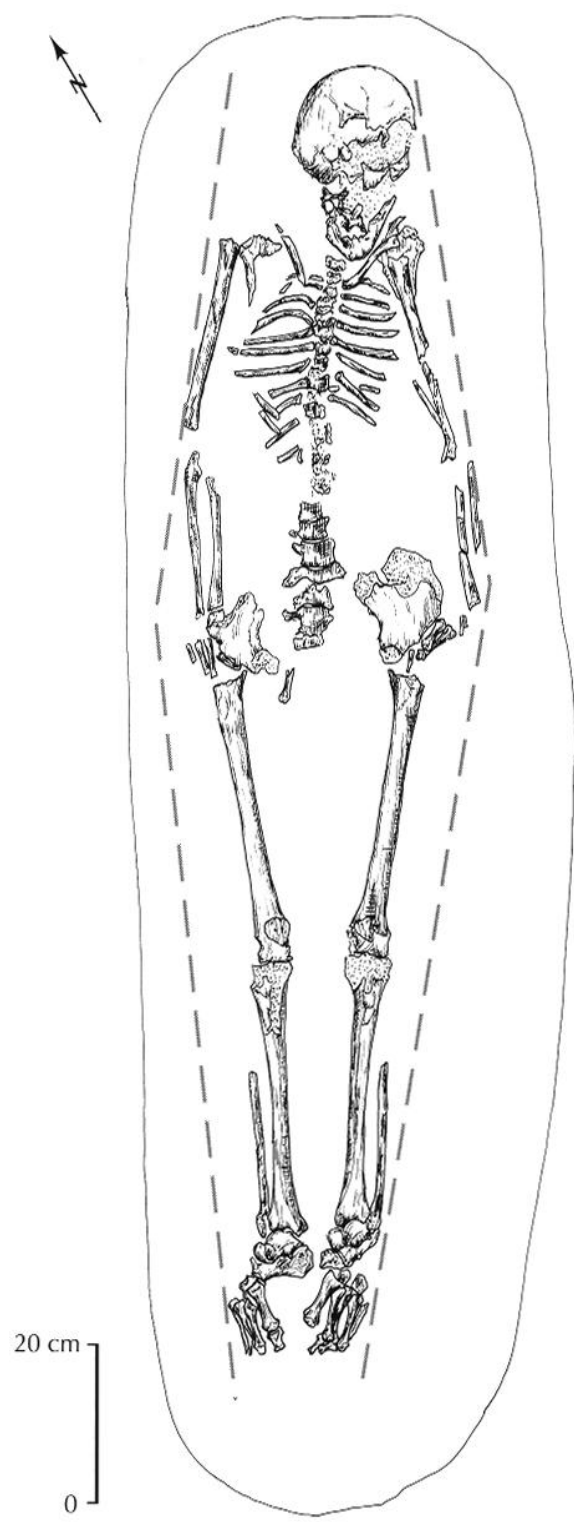

Fig. 15 - Le Trillet à Meyzieu, sépulture SP18. En pointillé : contraintes latérales permettant d'estimer la forme du conlenant (dessin: J. Cathalaa et F. Blaizot ; DAO: G. Macabéo). 
perpendiculairement à l'axe de la jambe. Ces effets de paroi sont dans l'ensemble parfaitement linéaires, l'emprise des pieds étant équivalente à celle des hanches et des épaules, ce qui prouve que le contenant était de forme rectangulaire. Celui-ci semble avoir possédé un fond, comme l'attestent l'importante mise à plat des côtes, la sinuosité et la segmentation en vrille de la colonne vertébrale.

La forme hexagonale est particulièrement bien représentée par le squelette de la sépulture SP18 (fig. 15). Le thorax présente un resserrement au niveau des épaules, accompagné d'une obliquité des clavicules. Ce resserrement s'exprime différemment de chaque côté : à droite, l'épaule est à plat et c'est le coude qui est dirigé vers l'extérieur; à gauche, la scapula est de chant (effet de contrainte) et l'extrémité proximale de l'humérus s'est déplacée en direction médiale. La mise à plat des volumes (côtes et bassin) est totale ; ce phénomène, conjugué à la disjonction temporo-mandibulaire pouvant indiquer que le bloc cranio-facial a basculé, constitue un argument en faveur d'une décomposition en espace vide. Le pied gauche semble avoir été contraint, puisque le tarse est en vue latérale. L'ensemble des effets de contrainte observés sur tout le squelette dessine un losange régulier, montrant que le contenant était de forme hexagonale. On remarque un redressement de l'extrémité médiale des côtes, témoignant d'un affaissement des parties latérales du thorax; cette particularité, déjà évoquée ci-dessus pour la sépulture SP16, permet de proposer l'hypothèse d'un fond constitué de deux planches parallèles.

\section{Le Pillon}

Les contenants non cloués du Pillon sont incontestablement de forme rectangulaire, en témoignent les effets de paroi et de délimitation linéaires sur les os, parfaitement parallèles au bord des fosses. Dans la sépulture SP515 (fig. 16) où l'hypothèse d'un contenant est argumentée par le maintien de chant des métatarsiens gauches (effet de paroi) à distance de la paroi de la fosse, les os des pieds se situent dans le prolongement de la limite la plus externe donnée par les humérus, à droite comme à gauche; le réceptacle ne se rétrécissait donc pas au niveau des pieds. Au Pillon, le mauvais état de conservation des ossements ne permet pas d'estimer précisément la largeur des contenants. Les effets de contrainte restent faibles, ils sont rarement bilatéraux et

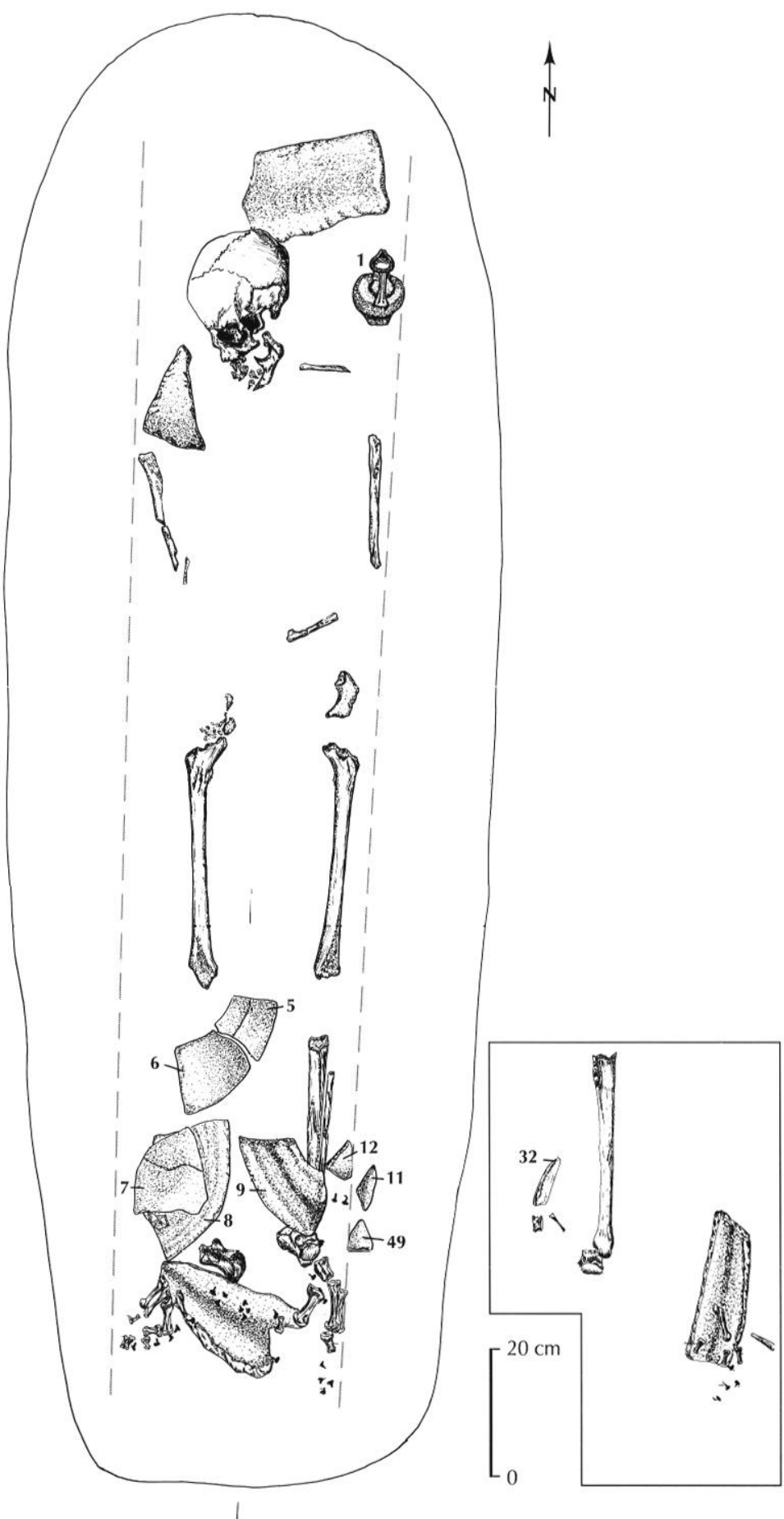

Fig. 16 - Le Pillon à Marennes, sépulture SP515. Les fragments de céramique $n^{\text {os }} 5,6,7,8,9,11,12,32$ et 49 appartiennenl au plat $n^{\circ} 2$ (cf. pl. IV). En pointillé : emprise du contenant (relevé et dessin : F. Blaizot; DAO : G. Macabéo). 


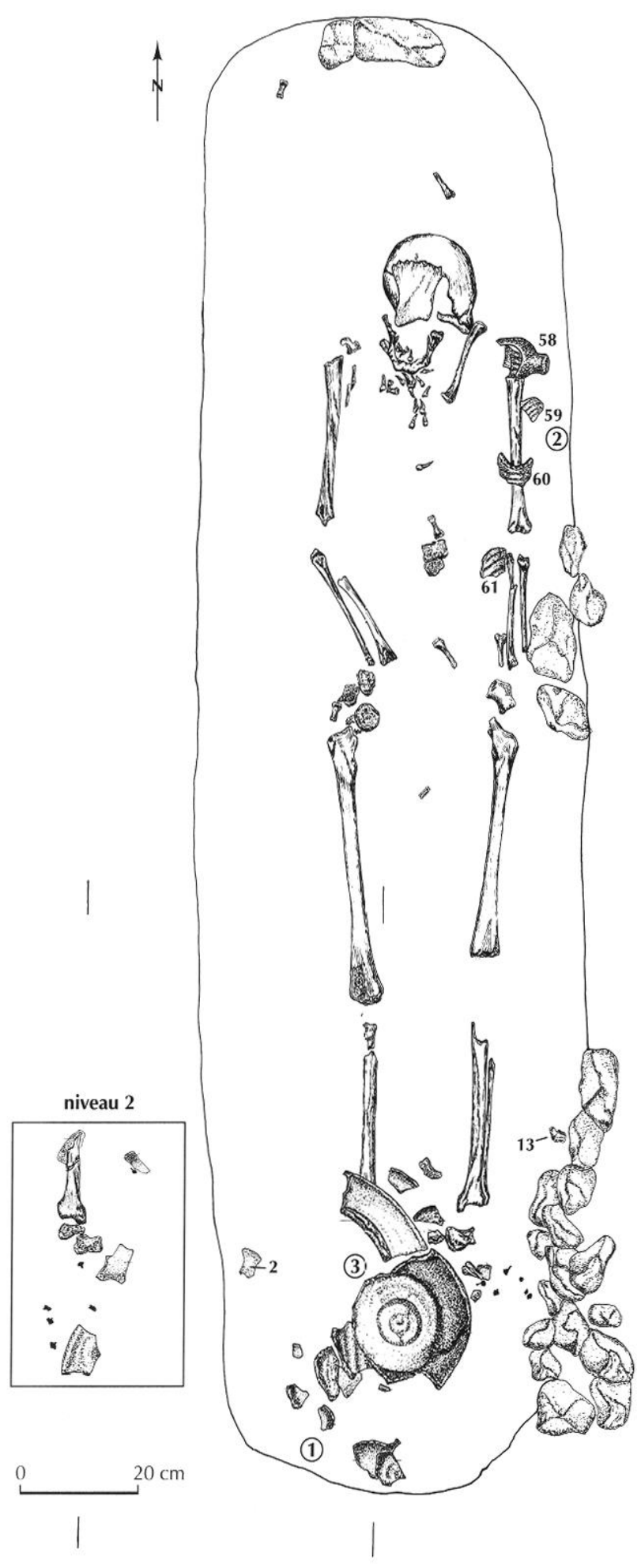

Fig. 17 - Le Pillon à Marennes, sépulture SP508. Les fragments de céramique $n^{\circ 5} 58,59,60$ el 61 appartiennent au gobelet $n^{\circ} 2$ (cf. pl. I el fig. 9); les fragments de céramique $n^{\text {s. }} 2$ et 13 appartiennent à la cruche $n^{\circ} 1$ (relevé et dessin : F. Blaizot ; DAO : G. Macabéo). l'hypothèse d'un réceptacle relativement large peut être retenue, les corps étant généralement plaqués contre l'une de ses parois. La longueur des contenants ne peut pas non plus toujours être estimée. Les os des mains qui se sont déplacés en amont du crâne du squelette SP508 (fig. 17) sont en suspension dans le sédiment et ce phénomène est probablement consécutif au passage d'un fouisseur à un moment où l'ensemble de la fosse était colmaté. En revanche, dans une autre sépulture du Pillon non présentée ici (SP513) le déplacement d'un métacarpien en amont du crâne du squelette n'a pu se

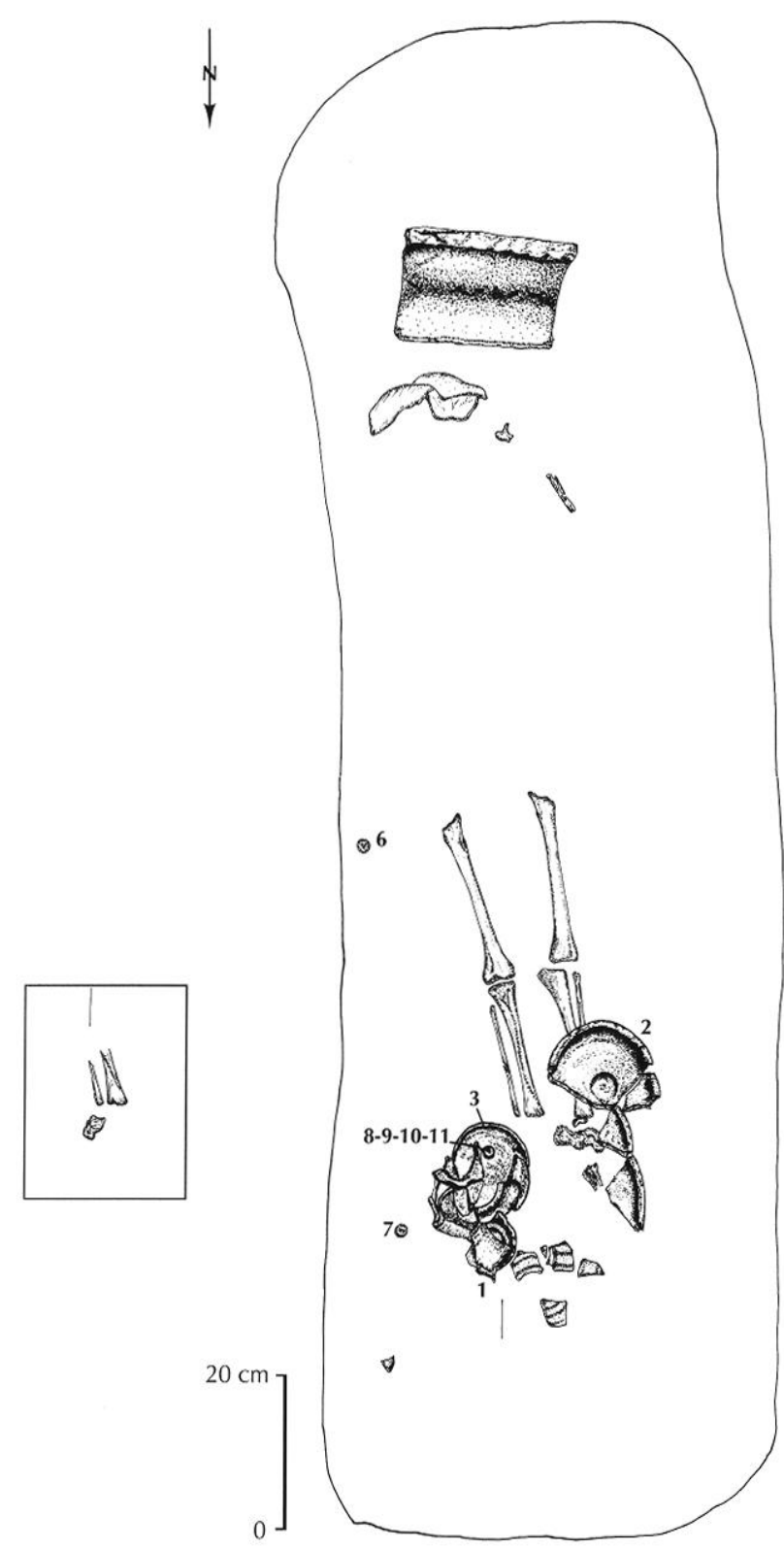

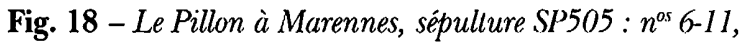
monnaies (relevé et dessin : F. Blaizot ; DAO : G. Macabéo). 
produire qu'en espace vide, puisqu'il repose sur le fond de la fosse, ce qui implique que le contenant était relati-

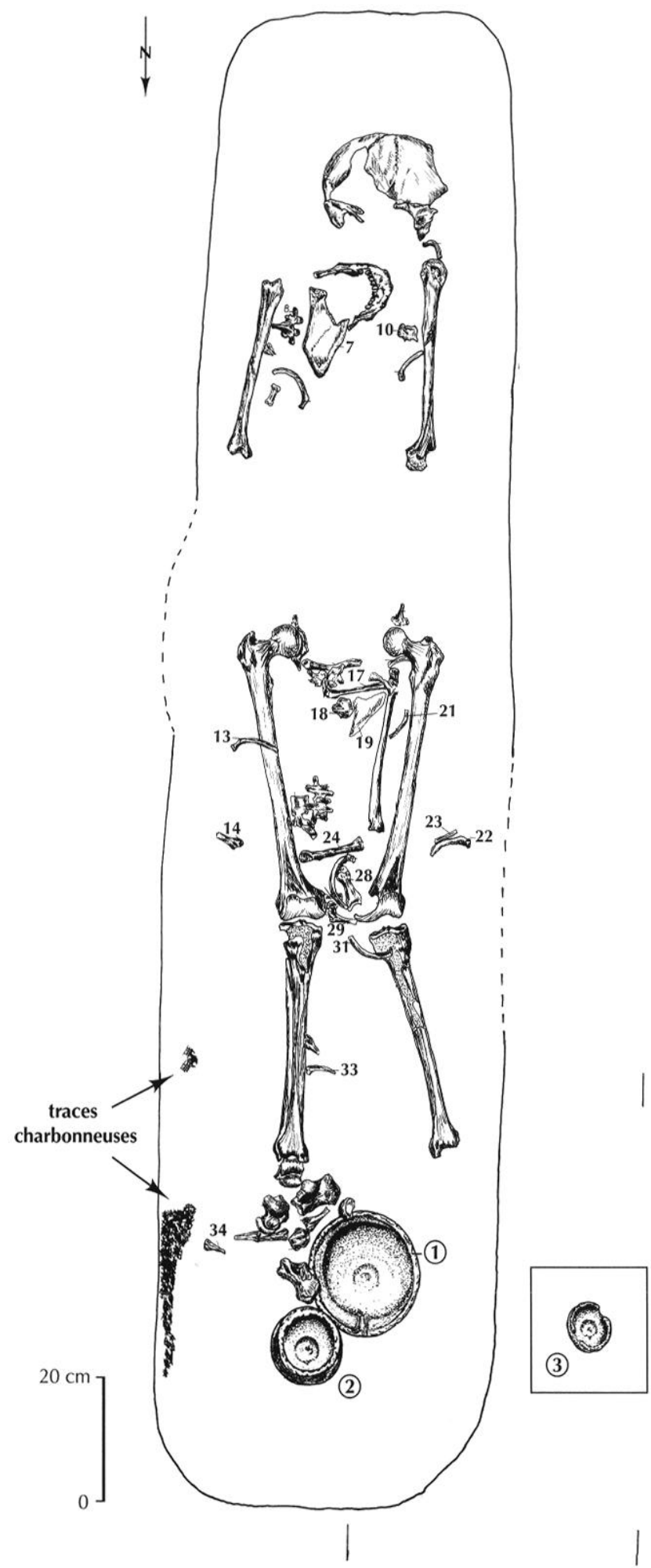

Fig. 19 - Le Pillon à Marennes, sépulture SP514 (cf. pl. IV). En pointillé : limites du contenant (relevé et dessin : F Blaizot; DAO : G. Macabéo). vement long. Pour les mêmes raisons de conservation, il est difficile de déterminer si les contenants possédaient ou non un plancher.

Dans trois cas cependant, l'hypothèse d'un plancher a pu être démontrée au Pillon, parce que les contenants reposaient sur des cales et non pas directement sur le sol. Dans deux sépultures (SP515, fig. 16 et SP505, fig. 18) ces cales sont représentées par des imbrices et des tegulae. tandis que dans la troisième (SP514, fig. 19) les cales étaient en matériau périssable.

Les effets d'alignement enregistrés sur le squelette de la sépulture SP515 permettent de restituer un contenant de bois (fig. 16). Dans cette sépulture, quatre éléments de calage ont été identifiés : un imbrex est situé en amont du crâne, un fragment de tegula se trouve au niveau de l'épaule droite et deux imbrices reposent à l'extrémité sud, sous les pieds du squelette. Un seul calage est observé dans la sépulture SP505 (fig. 18) ; il s'agit d'un imbrex, situé au sud, en amont des restes du bloc craniofacial. Au nord, le sol de la fosse remonte et sa cote de profondeur est équivalente à celle du sommet de l'imbrex placé à l'extrémité opposée. La partie postérieure du crâne et les pieds du squelette de la sépulture SP515 reposent directement sur les imbrices. La position du crâne, basculé vers l'avant et la droite, indique qu'il a glissé légèrement en aval de l'imbrex. Le même phénomène concerne les pariétaux et l'occipital du squelette SP505, puisque la distance qui sćpare ces éléments du segment moyen de la diaphyse humérale gauche est trop courte. Ces remaniements impliquent que ces morceaux de tuile se trouvaient bien sous ces régions anatomiques à l'origine et que la décomposition s'est produite en espace vide. Il apparaît donc justifié d'interpréter ces imbrices comme des cales inférieures du contenant. Celles-ci peuvent avoir été destinées à faciliter la récupération des cordes éventuellement utilisées pour descendre lc ccrcucil dans la fossc, mais la présencc d'une seule cale dans la sépulture SP505, expliquée par la morphologie du fond de la fosse, qui, parce qu'il remonte à l'extrémité nord, ne nécessitait pas l'adjonction d'une cale à ce niveau, va à l'encontre de cette proposition. Il est plus probable que ces imbrices aient été utilisées pour rétablir l'horizontale sous le cercueil.

Le squelette de la sépulture SP514 rend compte d'un grand nombre de perturbations, caractérisées par des déplacements d'ossements de grandes amplitudes, dont certaines se sont produites en dehors du volume initial 
du cadavre (fig. 19). Un contenant en matière périssable peut être déduit de l'effet de paroi qui s'est produit sur l'épaule gauche (humérus en vue postéro-latérale et décalé en hauteur) et des effets de délimitation linéaire illustrés sur le côté droit du squelette (alignement du coude, de la côte 13 et des métatarsiens 14 et 34). La présence de bois altéré contre la paroi est du tiers nord de la fosse confirme cette interprétation. Les remaniements ne sont pas alćatoires: la moitié supérieure se caractérise par des déplacements latéraux (une thoracique, 8 , se situe contre la diaphyse humérale droite, un fragment de la scapula droite est passé à gauche, 10, alors que son corps a glissé vers l'axe médian, 7) ; un grand nombre de pièces osseuses, situćes à l'origine dans l'étage supérieur du squelette, ont glissé en aval, entre les cuisses (vertèbres thoraciques, la scapula gauche, 19, les clavicules, 17 et 24 , et le radius gauchc), et les côtes comptent parmi les os qui ont subi les déplacements les plus importants (21, 13, 31, 22, 23, 33, etc.). Enfin, des os appartenant au tiers inférieur du squelette (métatarsiens $28,29,14$ et patella droite 18) ont effectué des mouvements vers l'amont par rapport à leur situation anatomique. Deux ruptures sont donc observées; l'une se définit par une zone vide en aval des coudes et par le déplacement des os de l'étage supérieur parmi les membres inférieurs. Ia seconde s'est produite sous le genou gauche, situé $5 \mathrm{~cm}$ plus profondément que la tête fémorale et que l'extrémité distale du tibia ; celle-ci est responsable du mouvement latéral du tiers distal du tibia et du déplacement en amont des os des pieds. Les déplacements latéraux n'ayant affecté que la moitié supérieure du corps, ils ne peuvent être révélateurs d'un contenant à fond concave. En revanche, les ruptures et les déplacements verticaux (axe longitudinal) pourraient correspondre à des fractures survenues sur le plancher d'un contenant, alors que la décomposition du corps était avancée et que le colmatage du squelette ne s'était pas encore produit; il est possible d'envisager une fracture médiane sous le thorax, une fracture transversale en aval des coudes et une dernière sous la jambe gauche au niveau du genou. Le fond de la fosse étant plat, ces fractures n'ont pu se produire que dans la mesure où le plancher du contenant était surélevé, probablement à l'aide de cales en matic̀re périssable, aucun imbrex n'ayant été relevé. On notera que la trace charbonneuse au nord-est repose $7 \mathrm{~cm}$ au-dessus du fond de la fosse: s'agit-il du niveau originel du plancher?
Les contenants de bois non cloués sont relativement mal connus, parce que leur identification, qui repose sur l'analyse des anomalies de position et de situation des ossements du squclette, a pendant longtemps posé des problèmes; on ne possède pas encore de corpus régional suffisant pour définir si les différences de morphologie et de construction, mises en évidence par l'anthropologie de terrain, ont une valeur chronologique. S'ils apparaissent à partir du II ${ }^{\mathrm{e}}$ s., leur utilisation se généralise surtout autour du IV ${ }^{\mathrm{e}} \mathrm{s}$. (Colardelle $e t$ al., 1996, p. 274) ; dans la basse vallée du Rhône, ils scmblent succéder au cercueil cloué à partir du Ve s. (Raynaud, 1987, p. 124), alors que plus au nord, on les rencontre dès le IV ${ }^{e}$ s. (Blaizot, Ayala, 1996) où les deux types - cloués/non cloués - coexistent (Ratel, 1977, p. 88 ; Privati, 1983, p. 56). L'emploi ponctuel d'un clou, pour parfaire la fixation d'un contenant assemblé d'une autre manière, a rarement été relevé jusqu'à présent ; nous l'avons rencontré néanmoins dans la nécropole de Saint-Irénée à Lyon (étude inédite F. Blaizot) autour des $\mathrm{V}^{\mathrm{e}}$-VIII ${ }^{\mathrm{e}}$ s., ainsi qu'en Alsace, dans des sépultures datées des Ve-VIIe s., dont des monoxyles (Blaizot, 1998a, p. 49 et 71). Certains contenants du Trillet étaient manifestement dépourvus de fond, ce qui permet véritablement de les interpréter comme des coffrages en planches réalisés in situ. Ccpendant, il peut exister des coffrages munis d'un plancher comme le prouvent les éléments de bois conservés dans les sépultures fouillées à Soyria, Clairvaux-les-lacs dans le Jura (Pétrequin et al., 1980). Sur ce site, les fonds sont constitués de planches disposées horizontalement, de la même manière que nous avons pu les restituer au Trillet à partir des anomalies taphonomiques (Pétrequin et al., 1980, p. 185). Au Pillon et au Trillet, les coffrages de planches sont associés à des contenants complets, et la présence de mobilier daté du IV e s., dans un cas comme dans l'autre, indiquerait que ces deux types de construction sont strictement contemporains. La même remarque concerne la morphologie de ces contenants - rectangulaires et trapézoïdaux - confirmant ainsi la coexistence de ces divers modes architecturaux dès l'apparition de ce type (Raynaud, 1987, p. 124; Colardelle et al., 1996, p. 274). Ainsi, contrairement à ce que l'on observe pour les sarcophages monolithes, où les formes trapézoïdale et rectangulaire sont utilisées de manière concomitante à partir du $\mathrm{V}^{\mathrm{e}} \mathrm{s}$. seulement (Colardelle et al., 1996, p. 280-281), les deux morpho- 
logies sont rencontrées plus tôt dans les architectures en matière périssable, parfois même dans les cercueils cloués (Ratel, 1977, p. 88).

Les coffrages de bois hexagonaux ne sont pas pour l'instant recensés dans la littérature. On connaît des formes ovales-hexagonales et strictement hexagonales, à des périodes plus tardives $\left(\mathrm{X}^{\mathrm{e}}-\mathrm{XI}^{\mathrm{c}} \mathrm{s}\right.$. et milieu

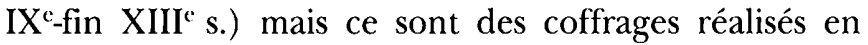
dalles courtes, en moellons ou en galets (types 12 et 13 de Colardelle et al., 1996). Nous avons néanmoins exhumé une sépulture de cette forme pour l'Antiquité tardive, située dans le Gard; elle est définie par un coffrage associant des tegulae, des dalles et des planches (site de La Ramière à Roquemaure, étude inédite F. Blaizot). Fn matériaux périssables, ils ne sont pas recensés avant l'époque moderne (Hunot, 1996, site de la place de l'Église à Écully dans le Rhône, étude inédite F. Blaizot).

Les contenants placés sur cales existent à toutes les périodes chronologiques. On en connaît des exemples dans l'Antiquité tardive, en pierre (Joly, 1991 ; Tranoy, Gisclon, 1995) ou en matériau périssable (Blaizot, 1998b).

\section{LES BÂTIÈRES DE TEGULAE}

Ce type de tombe ne concerne que deux sépultures exhumées sur le site des Girardes à Lapalud (SP5001 et SP5002). Nous l'illustrerons par l'exemple de la sépulture SP5002.

L'architecture est constituée d'un fond et d'une double rangée de tegulae disposées en bâtière sur les longs côtés, tandis que les extrémités sont fermées d'une seule tegula (fig. 20). Les tegulae placées dans le tiers est et celles du tiers ouest sont recouvertes d'un imbrex sur leur arête.

L'état de conservation du squelette est très médiocre. D'importants remaniements affectent la tête et la base du cou ; ils sont illustrés par la bascule du bloc cranio-facial en arrière, qui s'est dissocié de la mandibule et de la première cervicale. Aucune cervicale n'est en connexion anatomique, certaines sortent du volume initial du cadavre et les premières thoraciques sont disloquées. Enfin, le retournement des côtes supérieures droites témoigne d'un effondrement. Le coude droit est dissocié, puisqu'il est décalé en dehors par rapport à l'extrémité distale de l'humérus. Enfin, une dislocation affecte le genou droit : les deux os de la jambe ont glissé en direction médiale par rapport au fémur et sont venus buter contre le tibia gauche qui est en place dans le prolongement du fémur.

L'amplitude des remaniements indique que la décomposition du corps s'est effectuée en espace vide, aménagé par les parois de tegulae disposées en bâtière.

Les mouvements qui ont affecté le crâne, le cou et la partie supérieure du thorax droit témoignent d'une mise en déséquilibre brutale et d'un effondrement; ce mode de perturbation traduit un espace vide secondaire. La dissociation du genou droit pourrait relever d'un phénomène identique, ou bien avoir été causée par la situation initiale de la jambe, appuyée contre le rebord d'une coupe en céramique. Deux hypothèses sont ainsi envisagées: soit le corps reposait sur un support en matière périssable, soit le crâne et la partie supérieure du thorax seuls étaient placés sur un coussin. La première nous semble irrecevable compte tenu de la situation de la cruche et du pot qui se trouvent sur la fibula droite; elle supposerait en effet que les objets se trouvaient également sur l'éventuel support, ce qui n'est compatible ni avec leur disposition actuelle (ils ne témoignent d'aucun effondrement, reposent à plat sur le même axe) ni avec la position de la coupe qui n'a pas suivi la jambe droite dans son mouvement. Le déplacement de la jambe a donc plus probablement été causé par sa position originelle, en déséquilibre sur la coupe, tandis que seules la tête et la moitié supérieure du thorax reposaient sur un élément en matière périssable.

Les bâtières de tegulae sont utilisées dès le Haut-Empire, mais elles semblent se généraliser surtout entre les $\mathrm{III}^{\mathrm{e}}$ et $\mathrm{VII}^{\mathrm{e}} \mathrm{s}$., avec la plus haute fréquence au Ves. (Raynaud, 1987). Il s'agit d'une forme spécifiquement méridionale, de même que les coffrages rectangulaires de tegulae, qui n'est que ponctuellement rencontrée en amont de la Drôme (on en a quelques exemples dans l'Isère, dans le Rhône, en Suisse... où ils ne constituent qu'une part minime des types représentés). Sur la nécropole du Verdier, dans le Gard, les bâtières de "type soigné ", dont les joints sont couverts de tegulae et d'imbrices, caractérisent le $\mathrm{IV}^{\mathrm{e}} \mathrm{s}$., alors qu'au $\mathrm{V}^{\mathrm{e}} \mathrm{s}$. l'architecture est plus sommaire (Raynaud, 1987, p. 126) ; celle des Girardes, soigneusement construite, comporte bien du mobilier daté du IV s. (cf. infra). 


\section{LES FOSSES RUPESTRES EN ROCHE MEUBLE}

Ce terme défini par M. Colardelle (Colardelle et al., 1996) est employé pour désigner une fosse creusée dans un sédiment meuble qui reçoit directement le corps. Les méthodes de l'anthropologie de terrain ont permis de préciser leurs caractéristiques " architecturales ", pas toujours lisibles sur le terrain ; la fosse a généralement un aspect anthropomorphe, elle est resserrée aux deux extrémités et possède un profil concave, dit en gouttière.

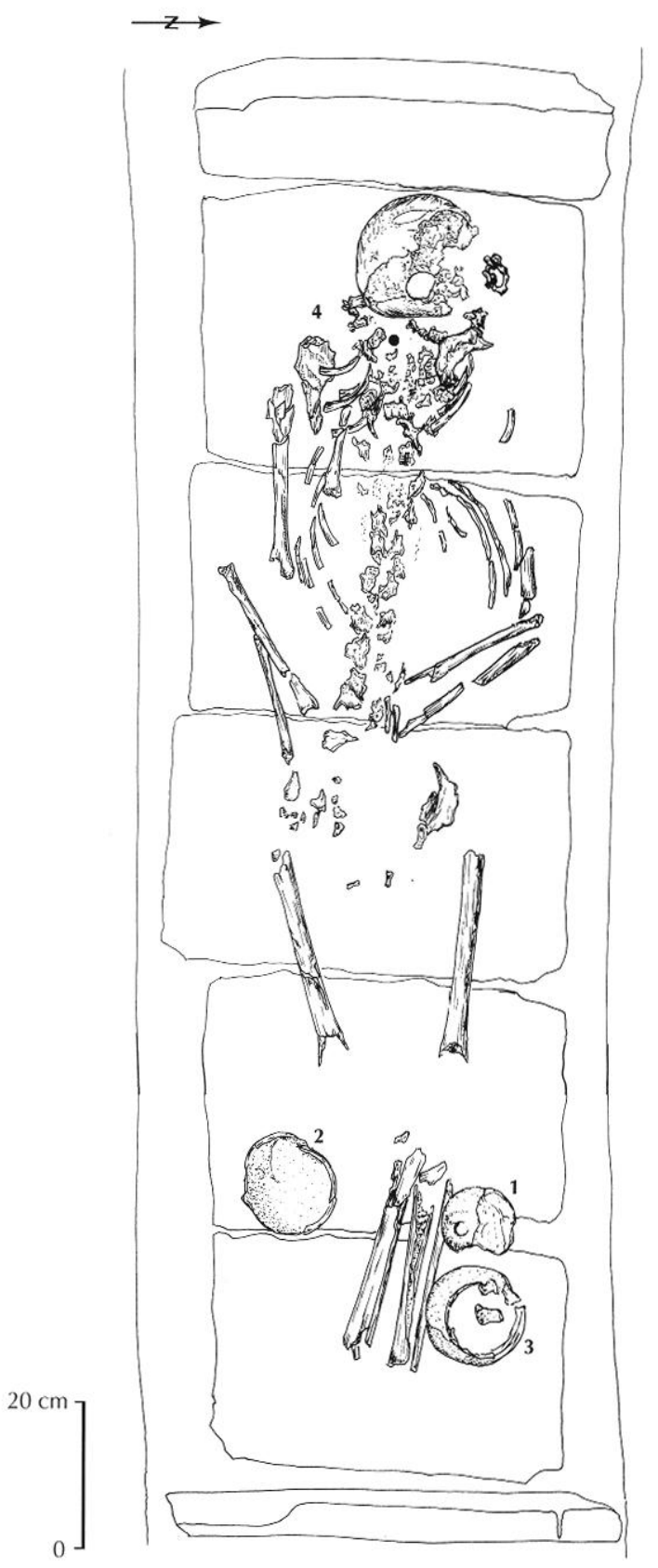

Le squelette présente les signes d'une décomposition en espace vide plus ou moins nets, dans la mesure où le creusement est en gouttière, ce qui a comme conséquence de limiter les déplacements latéraux sur les ossements placés en équilibre instable. Ces fosses possèdent un double creusement, la partie qui reçoit le corps étant plus étroite qu'en surface et le passage entre les deux s'effectuant par le biais d'une banquette. Elles sont parfois couvertes de dalles, mais dans la plupart des cas la fermeture est assurée par un élément en matière organique (planches?) ; cette couverture est parfois maintenue par des blocs de pierre.

Dans la série du Trillet, la sépulture SP9 peut être indiscutablement rattachée à ce type (fig. 21); elle comporte en effet deux fragments de dalle placés à l'extrémité est de la fosse au niveau des membres inférieurs du squelette sous-jacent, qui se situent à environ $37 \mathrm{~cm}$ au-dessus du fond de la fosse. À l'extérieur de la fosse, sur son bord sud, se trouve un gros galet de $22 \mathrm{~cm}$ de diamètre. Ces éléments paraissent désigner les restes d'un système de fermeture (couverture stricto sensu et calage) en supposant que la fosse ait été décapée jusqu'au niveau de la banquette. Certains mouvements effectués

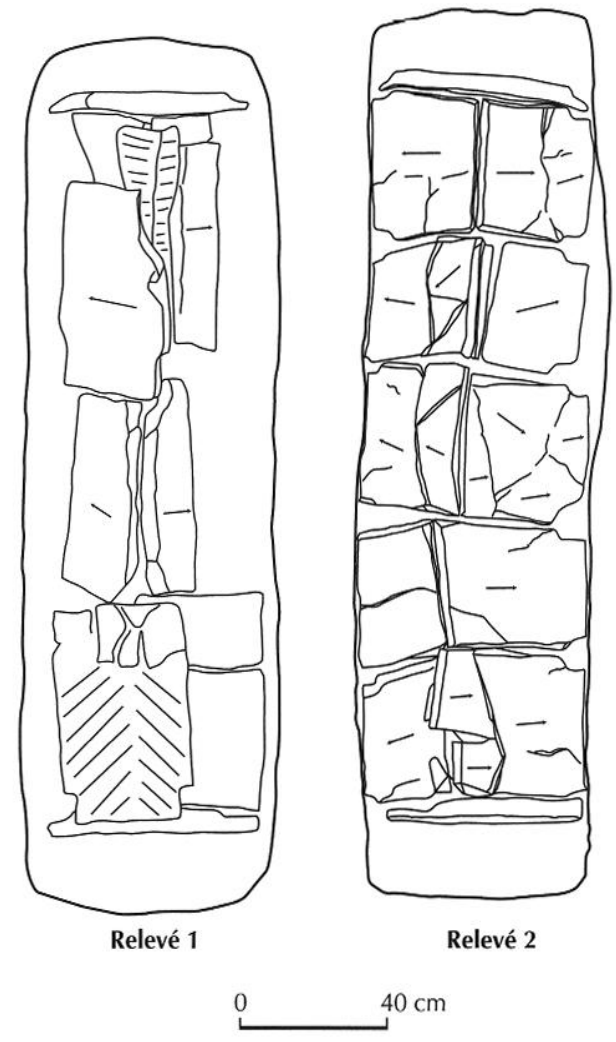

Fig. 20 - Les Girardes à Lapalud, sépullure SP5002 (cf. pl. VI) (dessin :

I. Blaizol; DAO:

G. Macabéo). 
par les os témoignent d'une décomposition en espace vide (dislocation du bloc cranio-facial, patella gauche située latéralement au tibia). La mise à plat des volumes est engagée, l'effet de gouttière reste faible ; il est toutefois induit par le fait que les humérus sont surélevés par rapport à la colonne vertébrale. La dislocation entre le bloc cranio-facial et la mandibule indique que le premier a tourné secondairement et ce mouvement, comme la rotation latérale du tarse gauche et la chute de la patella

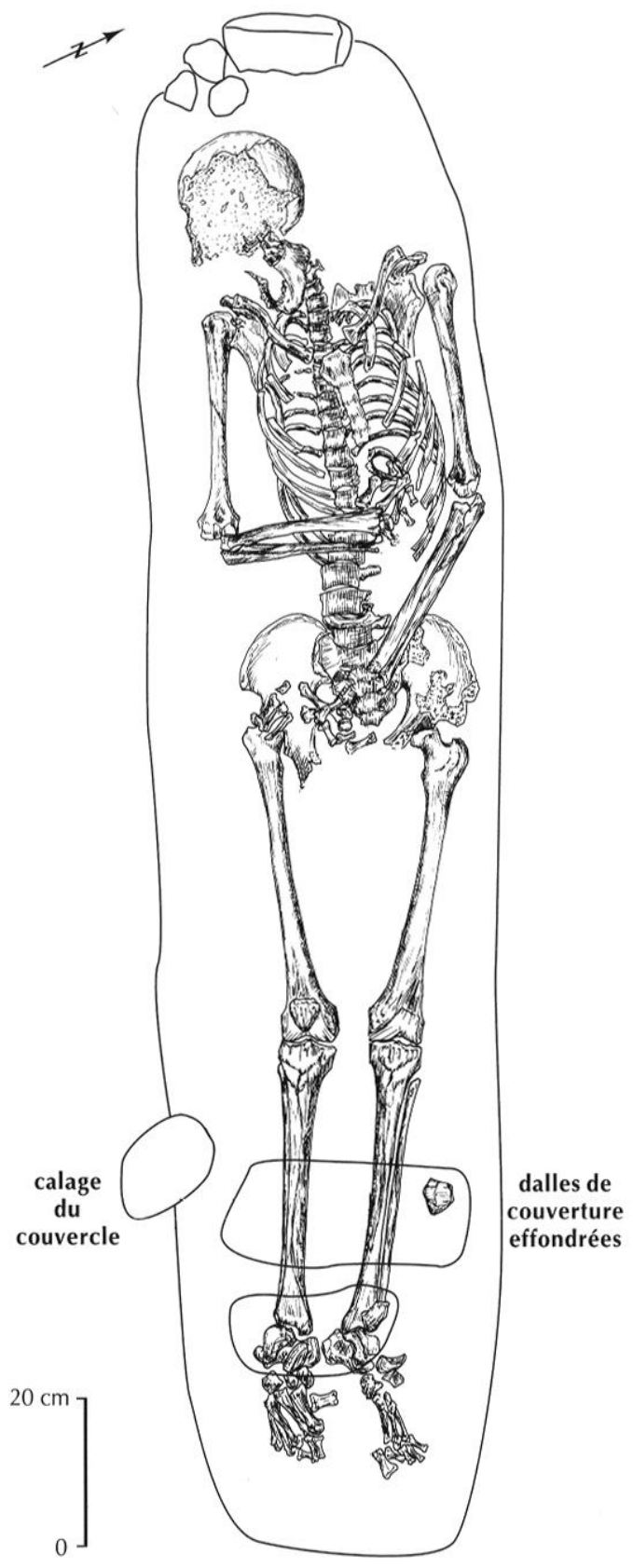

Fig. 21 - Le Trillet à Meyzieu, sépulture SP9 (dessin :J. Cathalaa et F. Blaizol; DAO: G. Macabéo). restent les seuls indices taphonomiques d'une décomposition en espace vide. On note que le crâne repose $8 \mathrm{~cm}$ moins profondément que le reste du squelette, ce qui implique que le sol de la fosse remonte à ce niveau selon la morphologie typique de ce type de sépulture.

Provenant du même site, trois autres squelettes présentent des caractéristiques taphonomiques qui évoquent un dépôt dans une fosse dont la zone médiane est surcreusée et délimite un espace restreint (SP2, SP3, SP5).

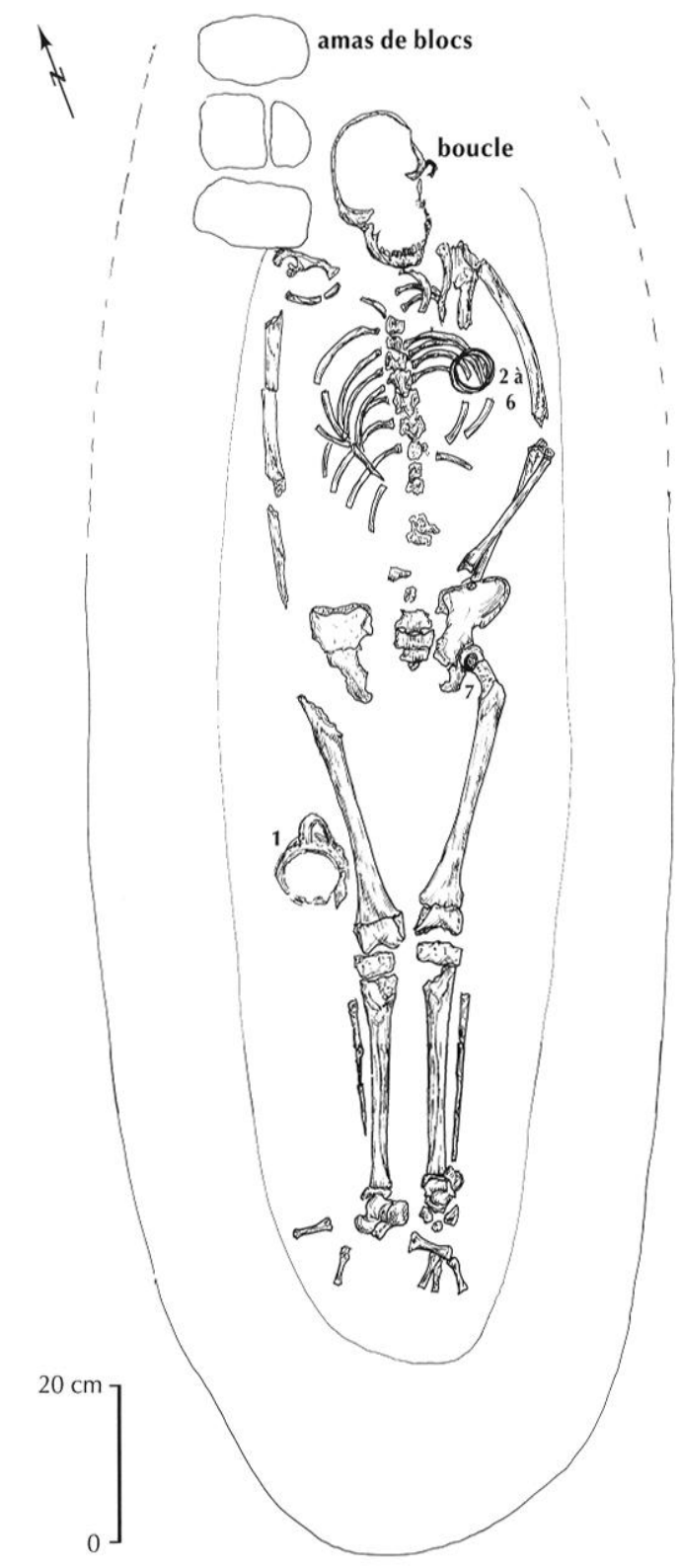

Fig. 22 - Le Trillet à Meyzieu, sépullure SP2 (dessin : J. Cathalaa et F. Blaizot ; DAO : G. Macabéo). 
Les anomalies relevées sur le squelette de la sépulture SP2 illustrent un creusement à double contour de la fosse (fig. 22). Toute la moitié gauche du squelette est légèrement décalée en hauteur par rapport à la droite. L'épaule gauche est surélevée et la scapula apparaît par sa face latérale. L'humérus, en abduction, est maintenu en vue latérale et l'attitude originelle de l'avant-bras, en pronation, est respectée. L'os coxal gauche ne s'est pas mis à plat, puisqu'il se présente en vue antérieure, ce qui traduit une contrainte latérale. À droite, l'humérus est resté en vue latérale et repose moins profondément sur le fond de la fosse que la colonne vertébrale. La scapula s'est redressée en vue antéro-supérieure. En amont de celle-ci, un amas de galets repose au même niveau que l'humérus. Les os des pieds sont disloqués en dehors du volume initial du corps, indiquant une décomposition en espace vide. Le métatarsien droit situć latéralement au pied est surélevé par rapport au fond de la fossc. L'effet particulier observé sur l'épaule gauche, la contrainte latérale sur l'os coxal gauche dont le bord externe se trouve en retrait par rapport au coude, la position de la scapula gauche et les cotes de profondeur relevées sous le bras droit et le métatarsien disloqué rendent compte d'un surcreusement du fond de la fosse avec un « effet de banquette ». L'origine des galets reste obscure ; le corps ayant évolué en espace vide, il n'est pas exclu qu'ils calaient le couvercle de la fosse et qu'ils se sont effondrés par la suite.

Dans la moyenne vallée du Rhône, les fosses rupestres en roche meuble sont pour l'instant rencontrées surtout au Moyen Âge classique (Colardelle el al., 1996, p. 293), mais une étude récente en recense de nombreux cas datés entre les VIII et $\mathrm{XI}^{\mathrm{e}} \mathrm{s}$. (Blaizot, Savino, à paraître). C'est d'ailleurs plutôt à cette période qu'elles sont connues dans les régions situćes plus au nord (Durand, 1988; Fréville, Journa, 1994), tandis que les sites de Dassargues à Lunel (Hérault) et de La Mérindole (Vaucluse) en livrent des exemples datés entre les VII et IX ${ }^{\mathrm{e}}$ s. (Garnotel, Raynaud, 1996, p. 150; Bellamy et al., 1996). À Strasbourg, nous en avons récemment exhumé une séric accompagnée de mobilier daté des dernières décennies du VII ${ }^{e}$ s. (Blaizot, 1998a). Dans les régions méditerranéennes, ces sépultures semblent cependant plus anciennes. Sur le site de Cadarache à Saint-Paul-lèsDurance (Bouches-du-Rhône) des fosses de ce type, couvertes de dalles, sont datées de la première moitié du V $V^{\mathrm{c}}$ s. au plus tôt (Pouyé et al., 1994); dans le Gard, sur le site de Font-du-Buis à Saze, ont été fouillées deux sépultures datées de la fin du $\mathrm{V}^{\mathrm{e}} \mathrm{s}$. au début du VII $\mathrm{s}$., dont les caractéristiques générales permettent de reconnaître ces fosses étroites à fond concave couvertes de dalles (Gagnière, Granier, 1972, p. 133 et fig. 4). Enfin, sur le site du Verdier à Lunel-Viel dans l'Hérault, C. Raynaud mentionne la présence d'une vingtaine de tombes dont la fosse présente un double creusement, le second épousant la forme du corps (Raynaud, 1987, p. 126-127). Les squelettes présentent les signes d'une décomposition en espace vide, ce que confirme la couverture de tegulae de ces fosses. Elles sont datées de la seconde moitié du $\mathrm{V}^{\mathrm{e}} \mathrm{s}$. Or, le mobilier associé à la sépulture SP2 du Trillet est daté de la seconde moitié du IV ${ }^{\text {e }}$ s. (tabl. I et inventaire, p. 342). On note que c'est la première fois que ce type de tombe est rencontré pour l'extrême fin de l'Antiquité tardive en dehors de la basse vallée du Rhône.

\section{LES INHUMATIONS EN PLEINE TERRE}

Ce mode d'inhumation n'est observé que pour des canidés qui proviennent de l'ensemble funéraire des Girardes. Il désigne une sépulture dans laquelle le cadavre a été directement recouvert de terre.

Les deux chiens paraissent avoir été déposés dans des fosses agraires, agrandies pour la circonstance, puisqu'elles se trouvent dans le prolongement exact des plantations antiques situées au nord (fig. 7). On précisera que cette constatation constitue le seul argument pour dater ces dépôts de l'Antiquité, puisqu'elle induit que les plants furent arrachés pour réutiliser la fosse. Il s'agit de chiens adultes, comme en témoigne le degré de maturation osseuse et dentaire, décédés après 3-4 ans (sutures inter-frontale et squamotemporale achevées). Dans les deux cas, compte tenu des difficultés licees à l'estimation du sexe chez les carnivores, seule l'absence d'os pénien indiquerait que ce sont des individus femelles. Ils semblent de tailles différentes, mais la variabilité interosscuse étant importante chez le chien, il serait nécessaire de le confirmer par une étude ostéométrique; cette hypothèse est en tout cas compatible avec ce que l'on sait des canidés à l'époque romaine, où on voit l'introduction de chiens bien différenciés morphologiquement (Cordy et al., 1995, p. 135).

L'un (SP5069, fig. 23) repose sur le côté droit et ses quatre membres sont réunis (les membres pelviens sont 


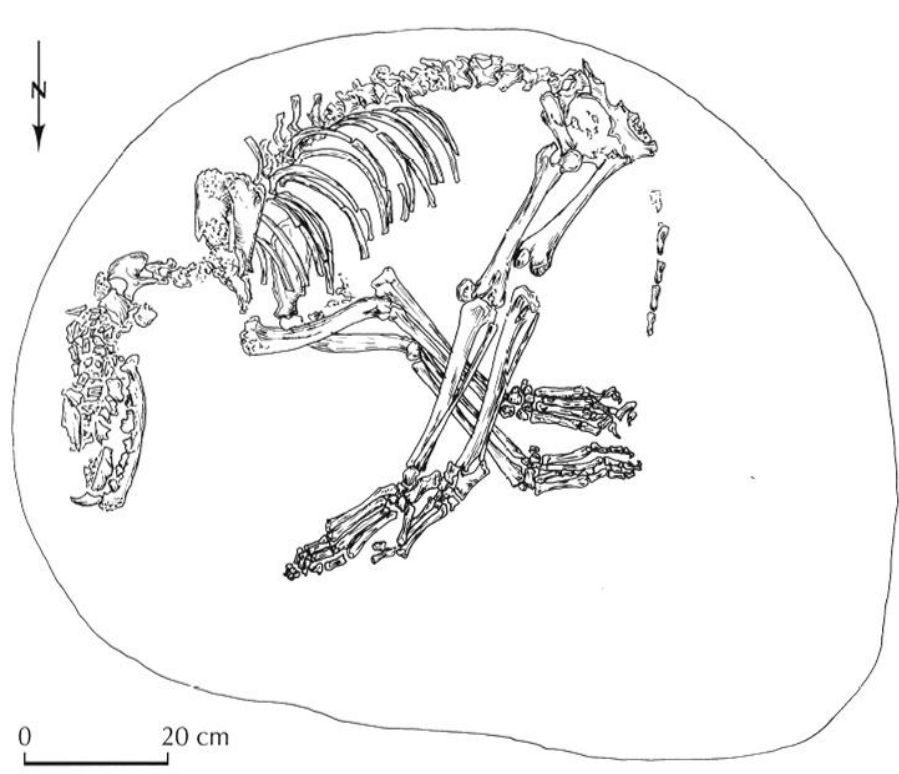

Fig. 23 - Les Girardes à Lapalud, sépulture de canidé SP5069 (dessin : F. Blaizol ; DAO: G. Macabéo).

orientés vers l'avant et les tibias et fibulas recouvrent les radius et ulnas dirigés vers l'arrière). Le second (SP5068, fig. 24) est placé sur son côté gauche, le cou en hyperflexion avec le crâne orienté vers l'arrière, la face inférieure de la gueule reposant en arrière de la région interscapulaire. Les connexions sont parfaitement maintenues, même sur les régions anatomiques placées en équilibre instable (cou, scapula droite, genou droit, pour SP5068). La décomposition s'est donc produite en espace colmaté, ce qui laisse envisager que les corps ont été directement couverts de terre. L'attitude de leur squelette ne reflète pas la posture naturelle d'un chien mort, que ce soit par la position de la tête et du cou chez l'un, ou par celle des quatre membres chez l'autre ; or, le maintien de ces positions n'est possible qu'au moyen d'un élément de contention et on peut envisager que ces animaux ont été ligotés, d'autant que l'attitude des membres de l'un d'entre eux (SP5069) rappelle celle du gibier que l'on transporte.

Les os ne portent aucune trace de blessure, mais le petit nombre de vertèbres caudales présentes dans la sépulture SP5068 indiquerait peut-être que sa queue a été coupée; toutefois, le reste des vertèbres ne se trouvant pas dans la fosse, rien ne prouve qu'elle ait été sectionnée à l'occasion de l'inhumation plutôt qu'à la naissance, comme cela se pratique aujourd'hui envers

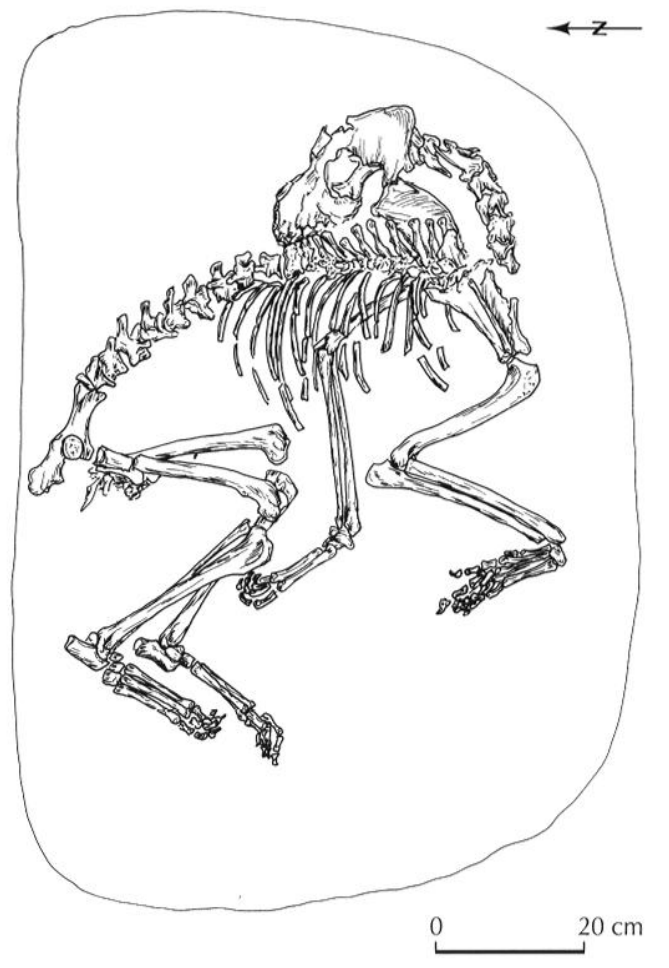

Fig. 24 - Les Girardes à Lapalud, sépulture de canidé SP5068 (dessin : F. Blaizot ; DAO : G. Macabéo).

certaines races pour des raisons esthétiques ou de sécurité (chasse au renard par exemple).

La présence de ces inhumations de chiens reste difficile à interpréter. Certes, il peut s'agir de véritables sépultures, mais également d'un moyen comme un autre de se débarrasser d'un cadavre. Leur situation au sein de l'ensemble funéraire est insuffisante pour démontrer que ces dépôts sont des sépultures, en l'absence de vestiges d'ordre cérémoniel. Les dépôts de chiens caractérisent plus particulièrement les cimetières des tribus germaniques dans les pays du Nord (Prummel, 1992). Ils sont moins fréquents dans le monde romain, où l'on distingue deux catégories (Lepetz, 1993) :

- soit la dépouille de l'animal fait l'objet d'une inhumation dans une fosse qui lui est propre, dans une nécropole ou non ;

- soit un squelette de chien, complet ou partiel, est retrouvé avec les restes humains, inhumés ou brûlés.

Dans le premier cas, même si la sépulture canine est située dans l'ensemble funéraire, rien ne prouve qu'elle participe au rituel funéraire ; il peut s'agir en effet d'un traitement donné au cadavre d'un animal familier, comme en témoignent certains exemples rencontrés 
d'inhumations de chiens accompagnés ou non de mobilier, pratiquées hors d'un contexte funéraire (Chastel et al., 1995 ; Mahé, Séguier, 1995 ; Pomarèdes, 1995). Par ailleurs, des inscriptions attestent des inhumations d'animaux dans les ensembles funéraires (Collectif, 1987, p. 38). La question est peut-être différente lorsque des inhumations de chiens sont pratiquées dans la structure funéraire qui contient le corps humain, tels les dépôts pratiqués au sommet des busta du HautEmpire (Bel, 1996) ou dans la fosse d'inhumation (site du lieu-dit Au Velours à Poligny, Jura : fouilles inédites de L. Staniaszek). Dans les cas où seuls quelques éléments du squelette du chien sont retrouvés, comme une patte (Lepetz, 1993, p. 40), l'hypothèse d'un sacrifice pratiqué à l'occasion de l'enterrement d'un humain est avancée, puisque le chien n'est pas, que l'on sache, un animal consommé par les Romains; l'ethnologie rapporte d'ailleurs de telles pratiques, comme chez les Hmong blancs en Asie du Sud-Est (Lemoine, 1979, p. 202). Sur le site des Girardes, l'interprétation de ces inhumations reste difficile; ces canidés sont-ils assimilés à du mobilier funéraire, auquel cas ils pourraient démontrer le prestige du défunt, ou représententils des guides symboliques pour "l'après-vie »? Cette seconde hypothèse a été privilégiée sur le site d'Argentomagus à Saint-Marcel (Indre) où des figurines en terre cuite, illustrant des animaux psychopompes, déposées dans les sépultures, coexistent avec de véritables sépultures de chiens (Allain et al., 1992, p. 112). L'éventualité d'un sacrifice des chiens des Girardes ne peut être proposée sur la seule base de leurs attitudes inhabituelles, celles-ci pouvant résulter du mode de transport des corps: dans un sac pour la sépulture SP5068 par exemple, porté " comme un gibier " dans le cas de la sépulture SP5069.

\section{MODE DE DÉPÔT DES CORPS HUMAINS}

Au Pillon, les fosses sont orientées nord-sud, avec une variante sud-est/nord-ouest, et aux Girardes, elles s'organisent sur l'axe est-ouest (une nord-est/sud-est). Au Trillet, la plupart des fosses sont orientées nord-nord-est et sud-sud-est, tandis que cinq, situées dans la partie sudest de l'ensemble funéraire, sont est-ouest. Tous les squelettes reposent en décubitus dorsal, attitude classique pour les périodes historiques.
Au Trillet comme aux Girardes, les squelettes placés dans les fosses orientées est-ouest ont la tête à l'ouest et les pieds à l'est. Si le crâne des squelettes qui reposent dans les fosses orientées globalement nord-sud est systématiquement situé au nord dans les sépultures du Trillet, le cas est différent au Pillon, où les deux positions (tête au nord et tête au sud) sont rencontrées. Dans cet ensemble funéraire, où les tombes s'organisent en petites unités ( $c f$. supra), l'orientation des corps est différente suivant le stade de maturation des individus et suivant les groupes spatiaux. Dans deux sous-ensembles (à l'est et au centre) les adultes sont déposés avec la tête placée au nord tandis que les immatures ont la tête au sud; dans le groupe situé à l'ouest, le phénomène est inversé, les adultes ayant la tête au sud et les immatures au nord.

Le dépôt de certains contenants sur cales, comme au Pillon, ne fait pas partie des gestes funéraires proprement dits, mais se rapporte aux modalités techniques de l'installation du cercueil. En revanche, dans la sépulture SP5002 des Girardes, la situation initiale de la tête et de la moitié supérieure du thorax du cadavre sur un élément en matière organique appartient au registre des gestes funéraires, puisque le dispositif intéresse directement le corps humain. Un coussin céphalique de ce type a également été reconnu dans la sépulture SP508 du Pillon; en effet, la migration de l'ensemble des dents vers l'avant témoigne d'une surélévation de la tête au départ et la situation du bloc cranio-facial, trop proche des épaules, indique un glissement de cet ensemble pendant la décomposition (fig. 17). Les coussins en matière périssable sont rarement mentionnés pour les sépultures de l'Antiquité tardive et ont surtout été observés dans des cas de conservation exceptionnelle des matériaux, comme à l'abbaye Saint-Victor à Marseille (Boyer, 1987) ; par ailleurs, il est fréquent que les défunts aient été déposés avec la tête placée sur un imbrex (Schuler, 1995, p. 110 ; Ferber, Ronco, 1996).

L'attitude des avant-bras a été analysée pour les séries du Trillet et du Pillon, les squelettes des Girardes ne fournissant pas suffisamment de données.

On note la quasi-exclusivité des positions basses (avant-bras en extension le long du corps ou ramenés en avant de l'abdomen) et moyennes (coude fléchi à $90^{\circ}$, avant-bras en avant sous la poitrine); seul le squelette SP513 du Pillon présente une position haute, avec la main gauche placée à la base du cou. Au Trillet, les avant- 
bras sont rarement situés en amont de l'abdomen chez les femmes et les enfants, alors que les hommes adoptent des attitudes plus diversifiées. Mais le petit effectif, conjugué à la présence majoritaire des hommes dans la série, ne nous autorise pas à émettre des conclusions certaines. On remarque toutefois que les individus placés dans des tombes adjacentes présentent la même position $(2,6,11 / 15,18,19 / 3,27,24 / 21,22)$. Ce phénomène reflète-t-il une pratique "familiale " ou une pratique momentanée, que les sujets soient regroupés selon leurs liens de parenté ou selon l'ordre dans lequel sont survenus les décès? Au Pillon, l'effectif observable est également trop faible (cinq). Les deux enfants (SP507 et SP504) ont les avant-bras ramenés en avant sur l'abdomen; ce sont les seuls à porter des bracelets et il est possible que cette attitude soit plutôt liée à la mise en évidence des bracelets plutôt qu'à l'âge des sujets.

\section{LE MOBILIER FUNÉRAIRE}

\section{LA VAISSELLE EN CÉRAMIQUE}

Dans cette étude, nous avons choisi de privilégier l'identification fonctionnelle au détriment du classement par type de production, celui-ci étant défini dans le tableau I et commenté dans le texte à l'intérieur de chaque catégorie fonctionnelle. Ce choix nous paraît mieux à même d'aborder le rituel funéraire, à travers les modalités de choix et de fréquence des vaisselles d'accompagnement et les associations de formes. En effet, pour les proches du défunt, la question est moins de déposer un vase à revêtement argileux ou une bouilloire en céramique commune rouge, que de répondre aux supposés besoins d'accompagner le défunt d'un vase à liquide.

$\mathrm{Au}$ total, quarante-cinq vases en céramique ont été déposés dans les sépultures des trois ensembles funéraires (tabl. I).

Six vases sont issus de six sépultures du Trillet (6 sépultures sur 32), vingt-sept vases de treize sépultures du Pillon (13 sépultures sur 15) et douze vases de six sépultures des Girardes (6 sépultures sur 15).

Chaque sépulture à dépôts du Pillon et des Girardes possède au moins un vase et un maximum de trois (entre un et trois vases), alors que celles du Trillet ont révélé un seul dépôt en céramique.
Sur l'ensemble des sites concernés par cette étude, $36 \%$ des sépultures avec dépôt en possèdent un seul, $44 \%$ en livrent deux et $20 \%$ en révèlent trois (inventaire).

\section{FONCTION DES VASES}

La plupart des auteurs traitant des dépôts céramiques en contexte funéraire font part de leur difficulté à classer strictement les vases à l'intérieur des catégories « vase à liquides " ou "vase à aliments solides " (Bel, 1992; Tranoy, 1995). S'il est aisé d'écrire qu'une cruche est un vase destiné à verser les liquides, un gobelet à boire et un plat à contenir des aliments solides, il s'avère plus risqué d'attribuer une fonction précise à toute une partie du vaisselier, qu'il s'agisse de céramique fine ou de céramique commune. Les pots en céramique commune grise peuvent tout aussi bien servir à cuire des aliments solides ou semi-liquides. Les petites coupes en céramique fine généralement interprétées comme des vases à boire contiennent à l'occasion des escargots ou des sauces (Bel, 1992). En l'absence de restes attestant un contenu original, nous utiliserons la classification fonctionnelle usuelle des formes déposées.

\section{ÉTUDE CÉRAMOLOGIQUE PAR FONCTION}

Les dépôts ont été classés dans les deux grandes catégories de céramique : la céramique fine destinée à la table, la céramique commune destinée à la cuisson des aliments solides ou semi-liquides ou à chauffer les liquides (tabl. I).

Les dépôts en céramique fine sont majoritaires dans chacun des ensembles funéraires, mais avec d'importantes variantes statistiques. Sur l'ensemble du Trillet, où nous raisonnons à partir de six vases seulement, la vaisselle de table est largement majoritaire avec un seul pot en céramique commune grise. En revanche, sur le site du Pillon $70 \%$ des dépôts sont en céramique fine, alors qu'aux Girardes la céramique commune occupe une part égale à celle de la vaisselle de table.

\section{La céramique fine}

Les céramiques fines (vingt-neuf dépôts), sauf un vase, sont des productions tardives à revêtement argileux (Lamboglia, 1963). L'état de la recherche ne permet pas d'attribuer systématiquement une origine et une période 
de production précises à ces produits fabriqués à partir des II'-III $s$. dans la moyenne vallée du Rhône et les Alpes du Nord (Desbat, Picon, 1986). Ils constituent l'essentiel de la vaisselle de table d'origine gauloise en usage dans les habitats et les nécropoles de l'Antiquité tardive de la Gaule du Sud.

Les céramiques à revêtement argileux du site des Girardes dans le Vaucluse ont probablement été fabriquées dans les ateliers voisins de la moyenne vallée du Rhône qui, à la fin de l'Antiquité, ont succédé aux fabriques de sigilléc claire B (Desbat, 1988). En effet, les dépôts des Girardes présentent des pâtes calcaires de couleur beige à orangée à l'aspect souvent savonneux, les revêtements sont assez. peu couvrants et varient de l'orange au brun.

Pour ce qui concerne les céramiques fines des deux autres ensembles proches de Lugdunum, il s'avère difficile de déterminer l'origine de chacun des vases. Certains ateliers alpins, dont le mieux connu est Portout à Chanaz en Savoie (Pernon, Pernon, 1990), possèdent une pâte qui se différencie des céramiques rhodaniennes tardives. Il s'agit d'une pâte rougeâtre, dure, avec un revêtement très couvrant de couleur rouge à brunc. Mais beaucoup d'autres produits ne présentent pas de caractères distinctifs aussi nets et ne peuvent être classés précisément. Aussi nous conserverons l'appellation " céramique finc à revêtement argileux " pour l'ensemble de ces productions et tenterons, dans quelques cas seulement, d'en préciser l'origine.

Dans le sud de la Gaule, le vaisselier de l'Antiquité tardive comporte également des vases fabriqués dans les provinces romaines d'Afrique, dont des plats en sigilléc comme celui déposé dans la sépulture SP515 du Pillon (Hayes, 1972 : type 50b).

\section{La céramique commune}

Sept dépôts en céramique commune sur quatorze sont à pâte grise (pâte siliceuse à postcuisson réductrice) dont un en argile blanche kaolinitique sur le site des Girardes. La céramique commune grise kaolinitique compose l'essentiel du vaisselier culinaire de la Drôme et du nord du Vaucluse (Alcamo, 1986). Il n'est donc pas surprenant de la retrouver en contexte funéraire.

Cinq autres dépôts sont à pâte rouge (pâte siliceuse à postcuisson oxydante). Cette production est également très représentative de la céramique régionale dans l'Antiquité tardive.

Deux des dépôts en céramique commune n'ont pu être attribués à une production précise, ils sont classés en céramique commune non identifiéc.

\section{Les vases à liquides}

\section{Les vases à verser des liquides}

\section{- Les cruches}

Parmi les vases en céramique fine, huit sont des cruches à revêtement argileux.

Quatre d'entre elles sont issues des modèles en sigillée claire $\mathrm{B}$ fabriqués depuis le début du $\mathrm{III}^{\mathrm{e}} \mathrm{s}$. et dont les formes perdurent au IV's. Il s'agit, en particulier, des formes Des. 84 dans la sépulture SP5002 des Girardes (pl. VI, SP5002 $\mathrm{n}^{\circ} 1$ ) et Des. 85 dans la sépulture SP15 du Trillet ${ }^{1}$ (pl. V).

Le type Des. 84 est présent dans les tombes SP147 (III ${ }^{\mathrm{e}}$ s.) et SP89 (IV s.) de la nécropole du quai Arloing à Lyon (Tranoy, Gisclon, 1995, p. 232-233) et dans la tombe SP12 (IV s.) de la nécropole du Patis I à Montboucher-sur-Jabron dans la Drôme (Blaizot, Ayala, 1996, fig. $\left.38, n^{\circ} 4\right)$.

Les types Des. 84 et 85 sont associés dans la nécropole de Saze dans le Gard datée de la fin du IV ${ }^{\mathrm{e}} \mathrm{s}$. (Gagnière, 1965, p. 68), dans la nécropole de La Brèche à Laudun également dans le Gard pour des sépultures datées du milieu du IV $\mathrm{e}$. (Feugère et al., 1987, p. 85), ainsi que dans la tombe SP89 du quai Arloing (Tranoy, Gisclon, 1995, p. 233).

La cruche de petit module de la sépulture SP11 du Trillet n'a pas d'équivalent exact (pl. V). Sa panse est piriforme, sa lèvre en bobine est connue parmi les productions de l'atelier de Portout en Savoie (Pernon, Pernon, 1990, pl. XVII, lèvre E), mais pas selon ce module.

I a sépulture SP515 du Pillon a livré une petite cruche à bec pincé en céramique commune rouge, l'anse à trois bourrelets est munic sur sa partie supérieure d'un bouton de préhension (pl. IV, SP515 n ${ }^{\circ}$ ). Ce type de vase est utilisé comme bouilloire, mais ce petit module n'est pas répertorié (Batigne, Desbat, 1996). Un modèle

1. La cruche déposée dans la sépulture SP1 du Trillet n'a pu être retrouvćc. Il s'agit d'une céramique à revêtement argileux, mais le dessin n'a pas permis de l'associer à un type précis. 
à bec pincé en céramique commune a été découvert dans la nécropole tardive de La Côte à Ambronay dans l'Ain (Monnoyeur, 1994, pl. 15).

\section{- Le tonnelet en céramique}

Un tonnelet en céramique à pâte fine micacée, de couleur rose, sans engobe, a été déposé dans la tombe SP5001 des Girardes (pl. VI). Les vases en céramique ou en verre s'inspirant de la forme du tonneau sont connus dès la fin du I ${ }^{\mathrm{er}}$ s. après J.-C. (Desbat, 1991, p. 330). Ils sont, entre autres, produits en sigillée à Lezoux dans le Puyde-Dôme (Bet et al., 1989, fig. 6, n 103) et en sigillée claire B (Desbat, 1994, fig. 3, nº 6). Il s'agit généralement de gobelets hauts ou de cruches; le tonnelet des Girardes se distingue de ces représentations puisqu'il est présenté sur le flanc dans la position usuelle du tonneau. D'une longueur de $100 \mathrm{~mm}$, d'une hauteur de $90 \mathrm{~mm}$ et d'un diamètre maximum de $90 \mathrm{~mm}$ (minimum $80 \mathrm{~mm}$ ), ce tonnelet repose sur quatre pieds. Le corps du tonneau est décoré de sillons réguliers alternant avec deux bandes d'environ $15 \mathrm{~mm}$ de large, décorées de chevrons. Sa partie supérieure est mutilée, mais elle comporte deux départs d'anses en ruban et un départ de col de cruche. La fonction de ce tonneau était donc de verser des liquides.

\section{Les vases à boire}

\section{- Les gobelets}

Les gobelets en céramique à revêtement argileux sont très bien représentés avec onze individus, dont deux dans deux sépultures du Trillet et les autres déposés dans pratiquement chaque sépulture du Pillon.

Dans la plupart des cas, ces gobelets sont à rapprocher du type Lam. 14/26. L'atelier de Portout a commercialisé un répertoire varié de gobelets avec ou sans anse ; afin d'actualiser le travail de N. Lamboglia nous nous référerons à la typologie proposée dans la publication de l'atelier de Portout.

Trois grands types ont été individualisés : le gobelet caréné, le gobelet tulipiforme, le gobelet à panse globulaire et col tronconique.

La tombe SP16 du Trillet comprend un gobelet caréné, sans anse, proche du type Lam. 14/26, à pâte orange et dure et revêtement orange à brun (pl. V, SP16 $n^{\circ}$ 2). Ce gobelet caréné est apparenté au type Portout 62, il est ici décoré de bandes guillochées horizontales. Le deuxième gobelet issu de la tombe SP10 du Trillet est de même type mais à lèvre en bourrelet, il peut être associé à la variante 130 du type 62 (pl. V, SP10 $\mathrm{n}^{\circ} 1$ ).

Les tombes SP504, SP507, SP509 et SP511 du Pillon ont livré, entre autres, un gobelet avec anse à panse tulipiforme de type Portout 66 (pl. II, SP504 $\mathrm{n}^{\circ} 2$ dont l'anse est absente, pl. III, SP507 $\mathrm{n}^{\circ} 1$; pl. III, SP509 $\mathrm{n}^{\circ} 1$; pl. IV, SP511 $n^{\circ} 1$ ). Le modèle découvert dans la tombe SP508 est proche du type Portout 65: un gobelet également tulipiforme mais sans anse.

Deux gobelets sont un peu plus hauts que les précédents et présentent une panse globulaire et un col tronconique plus ou moins important. Celui provenant de la tombe SP505 est sans anse et possède un col haut proche du type Portout 76 (pl. I, SP505 $\mathrm{n}^{\circ} 1$ ), tandis que celui de la tombe SP513 a un col plus court, une anse et s'apparente au type 75 de Portout (pl. IV, SP513 n ${ }^{\circ}$ 1). Le gobelet de SP5058 des Girardes est trop fragmenté pour être identifié.

Le gobelet à col haut de type Portout 76 est présent quai Arloing dans la tombe SP89 (IV s.), dans la tombe SP20 de la nécropole de La Boisse dans l'Ain ( $\mathrm{IV}^{\mathrm{e}} \mathrm{s}$.) (Vicherd, Baudrand, 1982, p. 136) et à Laudun, daté du milieu du IV $\mathrm{s}$.

Le type Portout 66 est également souvent déposé dans les tombes des nécropoles de Saze dans le Gard (Gagnière, 1965, fig. 13), de La Boisse et de La Côte dans l'Ain.

\section{Les vases à présenter les nourritures solides}

\section{- Les assiettes ou plats}

Cinq assiettes ou plats en céramique à revêtement argileux proviennent des trois ensembles funéraires.

Trois assiettes à fond plat et panse oblique sont à rapprocher du type Darton 40 ou Portout 1. Cette forme est attestée à La Côte et dans les nécropoles de la basse vallée du Rhône, à Saze (Gagnière, 1965, fig. 14, n 14), à Laudun (Feugère et al., 1987, p. 85, $\mathrm{n}^{\circ} 23$ ) où elle apparaît autour de 340 après J.-C.

Le plat à revêtement argileux provenant de la tombe SP5023 des Girardes est à fond légèrement bombé et lèvre à marli déversée ( $\mathrm{pl}$. VI, SP5023 n ${ }^{\circ}$ 2). Ce modèle est connu en sigillée claire B (Lam. 32) et attesté à Saze (Gagnière, 1965, p. $69 \mathrm{n}^{\circ}$ 13), au Patis (ibid., $\mathrm{n}^{\circ}$ 3) et dans une nécropole datée de la seconde moitié du IV ${ }^{\mathrm{e}} \mathrm{s}$., Fontager à Ponsas dans la Drôme (Richaud, Blanc, 1963, p. 85 , fig. 4 et p. 86 , fig. 8 ). 
Une assiette ou coupe à fond étroit annelé et lèvre tombante à marli guilloché, de type Lam. 4/36, a été déposée dans la sépulture SP509 du Pillon (pl. III, SP509 $\mathrm{n}^{\circ}$ 2). On retrouve ce modèle quai Arloing dans la tombe SP145 datée de la fin du $\mathrm{III}^{\mathrm{e}} \mathrm{s}$./début du $\mathrm{IV}^{\mathrm{e}}$ s. et à Laudun (Feugère et al., 1987, p. 87, $\mathrm{n}^{\circ} 1$ ).

Le vase déposé dans la sépulture SP512 du Pillon est également une assiette ou coupe à bord à marli (pl. IV, SP512 n 1). Celui-ci est mouluré et de facture assez grossière.

Nous noterons la présence dans la tombe SP515 d'un grand plat en sigillée africaine $\mathrm{C}$ de type Hayes 50B (pl. IV, SP515 $\mathrm{n}^{\circ}$ 2). Cette forme est une évolution tardive du plat Hayes 50, elle est datée de la seconde moitié du IV $\mathrm{IV}^{\mathrm{c}}$. Il s'agit de la seule importation recueillie sur les trois ensembles funéraires étudiés. Les céramiques africaines, vaisselle culinaire ou de table, sont fréquemment attestées dans les nécropoles du sud de la Gaule, comme celle de Laudun dans le Gard (sigillée africaine D), de Quarante et de Vias dans l'Hérault (africaine culinaire).

Cinq plats en céramique commune ont été déposés dans les sépultures. Leur fonction domestique est la cuisson des aliments (poêlons) ; en contexte funéraire, ils sont vraisemblablement destinés à présenter les nourritures solides.

Trois sont à pâte grise (pl. I, SP508 $\mathrm{n}^{\circ} 3$; pl. IV, SP514 $\mathrm{n}^{\circ} 1$ ), un est à pâte rouge (pl. IV, SP516 $\mathrm{n}^{\circ} 1$ ) et le dépôt de la sépulture SP511 est trop mal conservé pour être identifié.

Les plats en céramique commune sont associés aux céramiques fines dans la plupart des nécropoles aussi bien au quai Arloing (Tranoy, Gisclon, 1995, p. 235) qu'à Laudun (Feugère et al., 1987, p. 85).

\section{- Les coupes ou jattes}

Une coupe en céramique à revêtement argileux a été recueillie dans la sépulture SP506 du Pillon (pl. I, SP506 $\mathrm{n}^{\circ} 1$ ) ; ce vase caréné est à rapprocher du type Darton 19 ou Portout 24.

Les trois autres formes ouvertes sont des jattes en céramique commune à pâte rouge. Ces formes carénées sont bien connues dans les contextes d'habitat à partir du milieu du IV s., aussi bien en Rhône-Alpes qu'en Languedoc (Dicocer, 1993, COM-E-M B2). Certains auteurs en font des marmites, d'autres des plats de service.
Le dépôt de la tombe SP505 du Pillon a un profil proche de celui de la tombe 89 du quai Arloing (pl. I, SP505 $\mathrm{n}^{\circ}$ 2). Cette forme est également attestée dans la nécropole de Fontager dans la Drôme (Richaud, Blanc, 1963, p. 87).

Les deux vases des Girardes (pl. VII, SP5008 $\mathrm{n}^{\circ} 2$; pl. VI, SP5002 $\mathrm{n}^{\circ}$ 2) sont fréquents dans le vaisselier domestique de la Drôme (Bonnet, à paraître, fig. 15, $\mathrm{n}^{\circ}$ 10) et du Languedoc (Raynaud, 1990, p. 233, $\left.\mathrm{n}^{\text {os }} 13-14\right)$ à partir du milieu du IV $\mathrm{s}$.

\section{Les vases à présenter les nourritures solides,} liquides ou semi-liquides

\section{- Les coupelles}

Deux coupelles en céramique à revêtement argileux ont été déposées dans deux tombes du Pillon. Le dépôt de la tombe SP514 est à associer au type Portout 33 (pl. IV, SP514 $\mathrm{n}^{\circ} 3$ ), c'est la réplique exacte en petit module de la coupe carénée à lèvre déversée de la tombe SP505. Le dépôt de la tombe SP513 est également une petite coupe carénée à bandeau droit en céramique à revêtement argileux de type Lam. $3 / 8$ ou Portout 30 (pl. IV, SP513 n²).

\section{- Les pots}

Les quatre pots ovoïdes en céramique commune grise, dont un fabriqué à base d'argile kaolinitique, ont été déposés dans les tombes des trois ensembles funéraires.

L'exemplaire trouvé dans la sépulture SP2 du Trillet possède un col cintré, une lèvre déversée et une anse (pl. V, SP2 $\mathrm{n}^{\circ} 1$ ). La nécropole de Laudun a livré un pot en céramique à pisolithes dont le profil général est proche ; la sépulture contenant ce dépôt est datée de la seconde moitié du IV ${ }^{e} s$. ou du $V^{e} s$.

Le vase de la tombe SP514 du Pillon est à lèvre déversée simple, sans col marqué (pl. IV, SP514 n²) ; celui de la tombe SP505 possède une lèvre haute en amande marquée d'un sillon ( $\mathrm{pl}$. I, SP505 n 3). Ce modèle est attesté dans les contextes d'habitat de la seconde moitié du IV $\mathrm{IV}^{\mathrm{e}} \mathrm{s}$. : quartier Saint-Jean à Lyon (Ayala, 2000, fig. 27, $\mathrm{n}^{\circ}$ 95).

Le pot ovoïde à lèvre en bourrelet creusée d'une gorge, provenant de la tombe SP5002 des Girardes, est un vase fabriqué à base d'argile kaolinitique (pl. VI, SP5002 $\mathrm{n}^{\circ} 3$ ). D'une résistance exceptionnelle, les 
produits kaolinitiques sont très prisés pour l'usage culinaire pendant toute l'Antiquité et au Moyen Âge depuis le sud de Valence jusqu'au nord du Vaucluse. Cette forme est attestée dans la nécropole de GrangeNeuve à Allan, dans le sud de la Drôme, datée du IV $\mathbf{s}$. (Ferber, Ronco, 1996, SP1030) et quai Arloing dans la tombe SP147. L'exemplaire du quai Arloing est dit à pâte grise ; l'auteur ne précise pas s'il s'agit d'une argile kaolinitique. Dans le mobilier des fouilles d'habitat de la Drôme, cette forme n'apparaît que dans la seconde moitié du IV ${ }^{e} \mathrm{~s}$. (Bonnet, à paraître, fig. $12, \mathrm{n}^{\circ} 5$ ).

\section{DATATION DU MOBILIER CÉRAMIQUE}

L'analyse typochronologique des céramiques confirme une datation des $\mathrm{IV}^{\mathrm{e}}-\mathrm{V}^{\mathrm{e}} \mathrm{s}$. pour les trois ensembles funéraires. Il sera toutefois difficile, dans la majorité des cas, de préciser la datation à l'intérieur de cette fourchette. L'état de la recherche sur les céramiques tardives de la moyenne vallée du Rhône et les comparaisons morphologiques avec les régions méditerranéennes ne permettent généralement pas d'affiner les datations.

Cependant, pour ce qui concerne Le Pillon, la présence dans la sépulture SP515 d'une céramique d'importation datée de la seconde moitié du IV ${ }^{\mathrm{e}} \mathrm{s}$. et dans la sépulture SP505 d'une coupe carénée en céramique commune permet d'affirmer que ces tombes appartiennent plutôt à la seconde moitié de ce siècle.

$\mathrm{Au}$ Trillet, la belle facture du gobelet en céramique luisante de la sépulture SP16 pourrait indiquer une origine savoyarde de l'atelier de Portout qui est productif de la fin du IV $\mathrm{e}$. jusqu'au début du $\mathrm{VI}^{\mathrm{e}} \mathrm{s}$. Le pot de la sépulture SP2 serait également caractéristique des dernières décennies du $\mathrm{IV}^{\mathrm{e}} \mathrm{s}$. ou du $\mathrm{V}^{\mathrm{e}} \mathrm{s}$.

Des formes telles que les jattes carénées et le pot des tombes SP5002 et SP5008 des Girardes n'ont pas été diffusées en quantité avant le milieu du $\mathrm{IV}^{\mathrm{e}} \mathrm{s}$.

En conclusion, il est vraisemblable que sur les trois ensembles le mobilier céramique de plusieurs sépultures date de la fin du IV ${ }^{\mathrm{e}}$ s., voire du Ve $\mathbf{s}$. pour Le Trillet.

\section{LE MOBILIER MÉTALLIQUE ET LA PARURE}

Le mobilier non céramique (pl. I à VI) provient essentiellement des nécropoles du Pillon et du Trillet.
Aux Girardes il a été trouvé dans une seule sépulture (SP5023). Pour le même nombre de sépultures (six), le mobilier est plus diversifié au Pillon qu'au Trillet. Les matériaux retrouvés ne présentent pas de caractère précieux: il s'agit d'éléments en fer et en alliages cuivreux, ainsi que de verre. L'état de surface est moyen, la patine étant très altérée par endroits pour les alliages cuivreux ; la gangue de corrosion est moyenne à faible sur le fer. Aucune de ces pièces n'a subi de restauration ou de nettoyage approfondi.

Ces ensembles ne livrent pas d'arme comme c'est souvent le cas dans ceux de notre région, ainsi par exemple dans la nécropole de Saint-Martin-du-Frêne (Collectif, 1998) et celle d'Ambronay (M. Rolland, étude en cours).

La faible quantité d'objets par individu rattache ces ensembles aux habitudes funéraires du sud de la France plutôt qu'aux pratiques reconnues pour l'Antiquité tardive dans l'arc alpin, en Allemagne, dans le nord de la France ou en Angleterre.

\section{LES CLOUS DE CHAUSSURES}

Des clous de chaussures ont été trouvés au Trillet dans une sépulture d'enfant et une de femme; au Pillon dans une sépulture d'enfant, une de femme et une de sexe indéterminé. Contrairement à d'autres sites, aucune sépulture d'hommes n'en a livré (Crummy et al., 1993; Tranoy, 1995). Le faible échantillon ne permet pas de tirer des conclusions sur les modes vestimentaires locales.

\section{LES ÉLÉMENTS DE PARURE}

Les bracelets constituent les principaux éléments de parure de la série. Ils sont associés à la fois aux enfants et aux adultes, que ce soit au Pillon ou au Trillet où deux squelettes immatures portent des bracelets et une boucle d'oreille (?) pour l'un, et une boucle en fer pour l'autre (pl. V, SP2 $\mathrm{n}^{\circ} 2$; pl. V, SP27 $\mathrm{n}^{\circ} 1$ ). Les trois bracelets exhumés sur le site des Girardes à Lapalud pourraient appartenir à une femme (pl. VI, SP5023 nos 16-19). Ce type de bracelet est toujours associé à des femmes. Vingt exemplaires quasi complets et un fragment isolé ont été trouvés.

Les bracelets sont placés dans les sépultures par lots de trois au Pillon (pl. III, SP507 $\mathrm{n}^{\text {os }} 3$-5) et aux Girardes (pl. VI, SP5023 nos 16-19), de quatre au Pillon 
(pl. II, SP504 nos 5 a à 5c) ou de cinq au Trillet (pl. V, SP2 $\left.n^{\circ} 2\right)$.

Le type de bracelet fermé est présent uniquement au Trillet où il a été découvert en cinq exemplaires dans la sépulture SP2 (pl. V, n²).

Les bracelets trouvés dans le cimetière du Pillon sont tous ouverts et beaucoup plus variés que ceux du Trillet ou des Girardes.

Quatre types principaux peuvent être distingués :

$1^{\text {er }}$ type : ruban large, très plat, avec bossettes carrées ou circulaires et fermeture à crochet et perforation (pl. I, SP502/503 $\mathrm{n}^{\circ} 1$ et $\mathrm{pl}$. II, HS $\mathrm{n}^{\circ} 4$ ). Les décors, très proches dans les deux cas, sont constitués d'une alternance de fines incisions obliques régulières qui encadrent un bandeau central. Celui-ci offre un motif en vague sinueux ponctué d'ocelles.

$2^{e}$ type : ruban étroit, plus épais, fermeture à crochet et perforation (pl. I, SP502/503 $\mathrm{n}^{\circ} 2$; pl. II, HS nos 2-3 ; pl. II, SP504 $\mathrm{n}^{\circ} 5 \mathrm{a}$; pl. II, SP504 $\mathrm{n}^{\circ} 5 \mathrm{~b}$; pl. II, SP504 n ${ }^{\circ} 5 \mathrm{c}$; pl. III, SP507 $\mathrm{n}^{\circ}$ 5). Trois sous-types peuvent être distingués par l'analogie de leurs décors. Sur le premier, le décor est composé d'incisions verticales très rapprochées (pl. II, SP504 $\mathrm{n}^{\circ} 5 \mathrm{a}$ et $\mathrm{HS} \mathrm{n}^{\circ} 2$ ), sur le deuxième la surface est partagée en trois zones, les deux extrémités portant de simples incisions verticales et la zone centrale une alternance de cannelures obliques et d'ocelles (pl. II, SP504 $\mathrm{n}^{\circ} 5 \mathrm{c}$ et pl. III, SP $507 \mathrm{n}^{\circ} 5$ ), le troisième sous-type présente une alternance d'incisions obliques inversées et de courtes cannelures obliques pour SP502/503 $\mathrm{n}^{\circ} 2$ et $\mathrm{HS}^{\circ} 1$. Enfin, un quatrième sous-type (SP504 $\mathrm{n}^{\circ}$ 5b) présente deux simples rangées de cupules lc long des bords et se termine comme les précédentes par une perforation.

$3^{e}$ type : jonc non décoré, ouvert ou avec fermeture à crochet et perforation (pl. III, SP507 n ${ }^{\text {os }} 3$ et 4).

$4^{e}$ type: torsade à fermeture en crochet et boucle (pl. II, SP504 $n^{\circ}$ 3). Par son mode de fabrication ce bracelet se rapproche de la bague ou boucle d'oreille mise au jour dans la même sépulture (pl. II, SP504 n 13).

Des bracelets identiques sont régulièrement exhumés dans des nécropoles géographiquement proches. Dans l'Ain, ceux des cimetières d'Ambronay (ćtude M. Rolland en cours) et de Saint-Martin-du-Frêne (Collectif, 1998), dans le sud languedocien ceux de la nécropole de La Brèche à Laudun (Feugère et al., 1987) ou bien encore certains exemples suisses (nécropole de Kaiseraugst, cf. Martin, 1991) constituent des éléments typologiquement très proches pour les $I V^{c}-V^{c} s$.
Les bracelets, bien qu'exclusivement associés à des sujets immatures, paraissent de grande taille pour des poignets d'enfants. En effet, les diamètres des bracelets fermés varient de $47 \mathrm{~mm}$ à $53 \mathrm{~mm}$, voire $60 \mathrm{~mm}$, et les longueurs des bracelets ouverts sont de $168 \mathrm{~mm}$ à $183 \mathrm{~mm}$.

Cette remarque permet de s'interroger sur leur utilisation réelle: ont-ils été portés par ces enfants de leur vivant ou s'agit-il simplement de parure pour des enfants défunts? Dans le premier cas, ils pourraient être portés non pas au poignet mais sur le haut de l'avant-bras, voire autour du biceps pour les plus jeunes ou les plus graciles. Dans notre série, les observations réalisées in situ confirmeraient le port à l'avant-bras.

Les bracelets ne constituent pas vraiment un indice de datation très précis. S'ils sont très fréquents dans des sépultures du $\mathrm{IV}^{\mathrm{e}}-\mathrm{V}^{\mathrm{e}} \mathrm{s}$. dans la moitié est et le nord de la France, dans tout l'arc alpin, le long du Danube, en Rhénanie et jusqu'en Angleterre, ils se trouvent également dans des ensembles funéraires du $\mathrm{VI}^{e}$, voire du $\mathrm{VII}^{\mathrm{e}}$ s., dans ces mêmes régions. Certains types présents au Pillon ou au Girardes ont été recensés dans l'étude menée par Ellen Swift sur plus de 400 types de bracelets du IV $\mathrm{V}^{\mathrm{e}}$ s. après J.-C. dans les régions septentrionales de l'Empire romain. Cet auteur situe leur production au IV ${ }^{e}$ s. après J.-C. sans pouvoir préciser cette datation ; tout au plus évoque-t-elle une particulière popularité dc certains types dans la seconde moitié du siècle. Les joncs ouverts et non décorés (pl. III, SP507 $\mathrm{n}^{\circ} 3$ ) sont rares en Gaule lyonnaise et se rencontrent plutôt en Germanie, en Rétie et en Pannonie, sans datation précise au $\mathrm{IV}^{\mathrm{c}} \mathrm{s}$. après J.-C. (Swift, 2000, p. 127). Ils sont fréquents dans les tombes de l'Antiquité tardive et souvent associés à du mobilier du IV $\mathrm{e}$ s. après J.-C. ou des environs de 400 après J.-C. (Steiner, Menna, 2000). Selon ces auteurs, les joncs à fermeture en crochet et bouclc proviennent également des contextes du IV ${ }^{\mathrm{c}} \mathrm{s}$. (pl. III, SP507 n 4). Enfin, en Suisse, dans le sud de la Bavière, en Gaule et en Bretagne, les bracelets en bandeaux de tôle de bronze sont associés à du mobilier daté des deux derniers tiers ou de la seconde moitié du IV's.

Un autre élément de comparaison vient corroborer cette datation du IV's. Le bracelet $\mathrm{n}^{\circ} 3$ de la sépulture SP504 du Pillon à Marennes (pl. II, $n^{\circ}$ 3) est identique à trois bracelets provenant de la nécropole de Pont-deVidal à Combas (Gard). Or, dans ces deux nécropoles, ces mêmes bracelets sont associés à une lampe à crochet 
en fer. La fourchette chronologique proposée pour le mobilier céramique de la sépulture du Gard est la première moitié du $\mathrm{IV}^{\mathrm{e}} \mathrm{s}$. (Parodi et al., 1987). Pour E. Swift, ces bracelets torsadés à deux brins, aux extrémités en boucle et crochet ou à deux crochets, sont présents dans toute l'aire géographique de son étude et semblent apparaître plutôt dans la seconde moitié du IV $\mathrm{e}$. après J.-C. (Swift, 2000, p. 124).

Par ailleurs, M. Feugère propose une fourchette IV $\mathrm{V}^{\mathrm{e}} \mathrm{V}^{\mathrm{e}} \mathrm{s}$. pour le type rubanné des Girardes à Lapalud (pl. VI, SP5023 n $\left.{ }^{\text {os }} 16-19\right)$. Notons que le décor alternant groupes d'incisions verticales et métopes lisses (Swift, type A14) est extrêmement fréquent dans tout le sud de l'Angeleterre (plusieurs exemplaires sur le site de South Shields), mais également à Bâle (Suisse) au IV ${ }^{\mathrm{e}}$ s. après J.-C. (Swift, 2000, p. 129).

La forme générale et le décor alternant incisions obliques et ocelles de deux bracelets du Pillon (pl. II, SP504 $n^{\circ} 5 \mathrm{c}$ et pl. III, SP507 $\mathrm{n}^{\circ} 5$ ) sont très proches d'un exemplaire trouvé en Westphalie, sur le site de KrefeldGellep (Swift, 2000, fig. 191), daté aussi du IVe s.

Notre échantillon ne renferme aucun bracelet rubané ouvert, plat et étroit, à fermeture en crochet et perforation circulaire, ou de bracelets à extrémités en tête de serpent. Ces modèles sont très fréquents dans les inhumations germaniques, suisses, bourguignonnes ou anglaises, et leur absence traduit peut-être une variante "régionale».

\section{LES LAMPES EN FER}

Deux lampes en fer ont été mises au jour dans l'ensemble funéraire du Pillon à Marennes (SP504 et SP507), dont l'une est tellement lacunaire qu'elle ne peut être identifiée que par analogie avec l'autre. Toutes deux sont associées à des enfants (pl. II, SP504 n ${ }^{\circ}$ 8).

Fabriquées en une seule pièce, elles ont la forme d'une vasque hémisphérique munie d'un élément de préhension ou de suspension plat et tronqué (pl. II, SP504 $\mathrm{n}^{\circ}$ 8). La radiographie effectuée sur l'objet le plus complet permet de restituer un " manche " très court (20 mm de haut) surmonté de deux tiges latérales tronquées. Cette forme est sans doute très proche des trois lampes en fer à deux crochets recourbés trouvées à Tarquimpol (Moselle) ou de l'exemplaire conservé au musée de Troyes dans l'Aube (Hofmann, 1959).
Dans l'Antiquité, les lampes constituent un dépôt habituel en contexte funéraire. Toutefois, ce type de lampe en fer, fréquemment identifié comme louche ou simpulum, reste peu fréquent. Parmi les contextes funéraires examinés par $\mathrm{M}$. Feugère, une dizaine seulement est recensée en France, dont celles de Tarquimpol (Feugère, 1993). On les retrouve souvent en plusieurs exemplaires dans certaines nécropoles, comme celle du Pillon. Deux exemples de ce type ont récemment été exhumés à Saint-Priest (Rhône) dans un épandage d'habitat du IV $\mathrm{V}^{\mathrm{e}} \mathrm{s}$. (Ayala et al., 2000, p. 25). Ces deux lampes du Pillon viennent compléter une série peu nombreuse dans notre région.

Plus de la moitié des lampes du type de celles du Pillon sont issues de contextes funéraires datés du IV $\mathrm{s}$. après J.-C. : par exemple, première moitié du IVe $s$. pour Pont-de-Vidal (Combas), seconde moitié du IV ${ }^{e}$ s. pour Les Gravenasses (Combas), fin du IV s. pour la nécropole de Sierentz (Haut-Rhin). Les lampes trouvées à Saint-Priest (Rhône) peuvent être attribuées à la seconde moitié $d u \mathrm{IV}^{\mathrm{e}} \mathrm{s}$. après J.-C., grâce à la présence de céramiques de l'Antiquité tardive et de monnaies émises par la dynastie constantinienne, entre 341 et 361 après J.-C. (Cécillon, 2001). En revanche, deux exemplaires issus des musées de Troyes et de Tarquimpol restent dans une fourchette chronologique imprécise en raison du manque d'informations sur leurs contextes. Par référence aux éléments datés par ailleurs, il semblerait que les lampes du Pillon offrent une indication chronologique relativement fiable.

De manière générale, une part importante du mobilier métallique a été datée à l'aide de la céramique retrouvée dans les mêmes contextes. Depuis quelques années, les datations des céramiques ont été affinées, mais ces progrès n'ont pas forcément entraîné une révision de l'attribution chronologique du mobilier métallique qui leur était associé. Ceci doit nous inciter à la prudence pour établir une typochronologie précise de ces bracelets. Étude qui reste à mener pour les provinces du sud de la Gaule, en parallèle à celle d'Ellen Swift.

M. R.

\section{LES MONNAIES}

Le recours à la numismatique pour dater les couches d'occupation antique est souvent considéré comme 
peu pertinent. Cétte réticence s'accentue encore lorsque l'on s'appuie sur le monnayage tardif qui est caractérisé par sa pérennité dans les échanges de la fin de l'Antiquité voire du haut Moyen Âge. S'il est incontestable que des monnaies frappées dans la première moitié du IV $\mathrm{e}$ s. après J.-C. circulent encore dans la seconde moitié de ce même siècle et parfois jusqu'au $\mathrm{V}^{\mathrm{e}} \mathrm{s}$. (Depeyrot, 1982; Brénot, 1986), il n'en demeure pas moins que le circuit monétaire a toujours été régulièrement alimenté par de nouvelles émissions durant cette période. Ainsi, pour dater un niveau d'occupation ou un ensemble clos, il ne s'agit pas seulement de constater, par exemple, qu'une monnaie de Constantin I (307-337) côtoie un bronze de Maxime (383-388), mais il convient aussi de s'interroger sur l'absence de types monétaires postérieurs à la monnaie la plus récente du lot; on ne perd ou on ne dépose dans une tombe que les monnaies que l'on possède à un moment donné. De plus, le degré d'usure des unités inventoriées doit également être apprécié car il peut révéler une circulation brève ou longue.

Par son homogénéité chronologique, le numéraire recueilli en contexte funéraire tardif semble être un indice de datation à ne pas négliger.

L'analyse du mobilier monétaire issu de dix-sept nécropoles tardives languedociennes, réalisée dans le cadre d'un travail universitaire (Pellecuer, 1986 ; Feugère et al., 1987, p. 84-85), a mis en évidence que l'écart maximal des émissions des monnaies placées dans une même sépulture n'excédait pas trente années et que plus du tiers d'entre elles n'était séparé que par vingt années seulement. Il apparaît donc que les dépôts monétaires ont été effectués relativement peu de temps après l'émission des monnaies les plus anciennes du numéraire recueilli dans les sépultures.

Le même constat, accentué, peut être établi après l'examen des lots monétaires livrés par ccrtaines sćpultures des nécropoles du Pillon et des Girardes pour lesquelles on note une grande homogénéité des dépôts monétaires multiples.

Hormis les deux monnaies illisibles (imitations radiées de l'extrême fin du III $^{c} s$. après J.-C. ?), et non exploitables, de la nécropole du Trillet (SP2 $n^{\circ} 22$ et SP30 $n^{\circ} 23$ ), les monnaies inventoriées au Pillon et aux Girardes permettent d'affiner la datation des sépultures qui les contenaient et aussi de préciser la fourchette chronologique de l'occupation d'une de ces nécropoles : celle du Pillon. En outre, le caractère des dépôts nous renseigne sur certaines pratiques funéraires liées à l'usage et à la symbolique de la monnaie.

On relève, tout d'abord, sur ces trois sites, que peu de sépultures contenaient des monnaies. Ainsi, dans la nécropole du Pillon, quatre sépultures sur quinze fouillées (soit $26,6 \%$ des tombes) renfermaient au moins une monnaie. Deux sépultures sur dix-sept (soit $12 \%$ des sépultures) de la nécropole des Girardes II avaient fait l'objet de dépôts monétaires ; il en va de même dans celle $\mathrm{du}$ Trillet, mais pour un total de trente-deux sépultures (soit un taux très faible de $6,25 \%$ des sépultures).

Dans la nécropole du Pillon, la sépulture SP505 contient six monnaies : deux unités sont placées latéralement au membre inférieur droit (SP505 $\left.\mathrm{n}^{\mathrm{os}} 6,7\right)$ et quatre monnaies sont empilées dans un pot en céramique commune grise (SP505 ${ }^{\text {os }} 8-11$ ). Ce lot monétaire se caractérise par la grande homogénéité chronologique de ses émissions, puisque les six monnaies d'époque constantinienne ont été frappées entre 312 et 315 après J.-C. On recense, en effet, cinq folles et demifolles à l'effigie de Constantin I (307-337) au revers SOLI INVICTO COMITI (SP505 nos 6-10) et un follis à l'effigie de Licinius I (308-324) dont le revers porte la légende GENIO POP ROM (SP505 $\left.\mathrm{n}^{\circ} 11\right)$. Ces six monnaies issues de l'atelier monétaire de Lyon, très proche du site, n'ont visiblement pas circulé : les moindres détails de leurs gravures sont, en effet, perceptibles (finesse apparente des mailles de la cuirasse de Constantin et des chevelures des deux empereurs). Le mobilier céramique mis au jour dans la sépulture SP505 indiquerait plutôt, mais de manière large, que l'inhumation serait intervenue au cours de la seconde moitié du $\mathrm{IV}^{\mathrm{e}} \mathrm{s}$. Or, le dépôt monétaire semble contredire cette datation: le numéraire semble avoir été déposé peu de temps après sa frappe. Il est, en effet, peu probable qu'autant de monnaies émises au début du $\mathrm{IV}^{\mathrm{e}} \mathrm{s}$. et provenant du même atelier (les pièces de Constantin ont été frappées dans la même officine) aient été encore en circulation après 350 après J.-C. sans être associées à des unités frappées sous la dynastie constantinienne entre 330 et 361-363 après J.-C. (revers GLORIA EXERCITVS et surtout FEL TEMP REPARATIO, cf. RIC, 1981) ou à des monnaies de la dynastie valentinienne entre 364 et 380 (revers GLORIA ROMANORVM ou SECVRITAS REIPV$B L I C A$, cf. RIC, vol. IX) dont les productions ont été massives et que l'on trouve en quantités importantes sur 
les sites tardifs régionaux (Brénot, 1986). À titre d'exemple, la nécropole tardive de Sainte-Colombe (Rhône), occupée durant toute la seconde moitié du $I^{e} \mathbf{s}$. jusqu'au début du $V^{e} s$., a livré onze monnaies dont les émissions s'étirent entre 330 et 392 après J.-C. (Audra, 1997).

En revanche, la présence d'une maiorina fleur de coin de Magnence (350-353) datée de 353 après J.-C. dans la sépulture SP502/503 (SP503 $\mathrm{n}^{\circ}$ 12) et d'un aes 2 de Maxime (383-388) dans la sépulture SP512 ( $\left.n^{\circ} 2\right)$ confirment que l'implantation des sépultures s'est échelonnée durant la seconde moitié du IVe s. Les monnaies mises au jour dans les niveaux d'occupation de la nécropole (US 200, US 617) attestent aussi la fréquentation des lieux durant la seconde moitié du IVe s. ( $c f$. inventaire, p. $350, \mathrm{n}^{\text {os }} 1,3$ et 4 ).

Ainsi, si l'on tient compte du dépôt monétaire de la sépulture SP505, on peut penser que la nécropole du Pillon a vraisemblablement été occupée dès les premières décennies du $\mathrm{IV}^{\mathrm{e}} \mathrm{s}$. et au moins jusque vers les années 380-400 après J.-C.

Le numéraire de la sépulture SP5008 de la nécropole des Girardes présente des similitudes avec celui de la sépulture SP505 du Pillon, hormis la période d'émission des monnaies. Elle contient, en effet, un lot de huit monnaies de bronze caractérisé par sa grande homogénéité. Trois monnaies sont empilées près du centre de la fosse (SP5008 $\mathrm{n}^{\text {os } 14-16)}$ et cinq pièces forment une pile à côté de la cruche $\mathrm{n}^{\circ} 1$ (SP5008 $\left.\mathrm{n}^{\text {os }} 17-21\right)$ : les huit monnaies reposent sur le fond de la fosse. Il s'agit de maiorinae ou de doubles maiorinae frappées aux effigies de Magnence (350-353), Décence (351-353) et Constance II (337-361). Les monnaies de Magnence et de Décence sont fleur de coin; elles ont toutes été frappées en 353 après J.C. lors de la dernière émission monétaire des deux usurpateurs gaulois. Le revers de ces monnaies à la légende SALVS DD NN AVG ET CAESAR figure systématiquement la représentation d'un grand chrisme accosté de l'alpha et de l'oméga (A et $\Omega$ ) (SP5008 $\left.\mathrm{n}^{\text {os }} 14,15,17-21\right)$. En apposant sur leur monnayage ce symbole chrétien, les deux adversaires de Constance II pensaient rallier à leur cause (déjà perdue) des partisans chrétiens au détriment de leur rival (Wigg, 1999). Les monnaies de la sépulture SP5008, aux noms de Magnence et Décence, proviennent principalement d'Arles et de Lyon; elles sont donc issues d'ateliers proches du lieu de leur enfouissement, ce qui raccourcit la durée de diffusion de ce monnayage. L'unique monnaie de Constance II recensée dans la tombe SP5008, au type FEL TEMP REPARATIO (l'empereur en habit militaire perçant d'une haste un cavalier sassanide désarçonné) ne rompt pas l'unité chronologique de ce dépôt monétaire funéraire (SP5008 ${ }^{\circ} 16$ ). Ce type monétaire, dont les émissions se sont succédé au fil des réformes pondérales sous le règne dominant de Constance II, a été frappé dans seize ateliers occidentaux et orientaux entre 346 et 361 après J.-C. (RIC, 1981). Notre exemplaire provient semble-t-il de Trèves. La frappe de cette pièce ne peut être postérieure à la réforme pondérale de 353 après J.-C., en raison de son poids (taille au 1/132 de la livre) et de son large module (Depeyrot, 1987, p. 105). Cette monnaie ne présente pas, a priori, de trace de circulation. De nouveau, l'homogénéité chronologique, malgré le nombre important de pièces, nous incite à penser que le numéraire présent dans la tombe SP5008 a été déposé peu ou très peu de temps après son émission, au milieu $\mathrm{du} I V^{\mathrm{e}} \mathrm{s}$.

En revanche, l'unique nummus de Constant (337-350) frappé entre 340 et 341 et recueilli dans la tombe SP5002 $\left(\mathrm{n}^{\circ} 13\right)$, s'il précise au moins le terminus ante quem du dépôt funéraire, ne permet pas de cerner précisément la période durant laquelle le défunt a été inhumé. En l'absence d'autres unités, on ignore, en effet, à quel moment cette petite pièce de bronze de la dynastie constantinienne a été soustraite du circuit monétaire. Cependant, on peut noter qu'elle n'est pas usée et qu'elle provient, elle aussi, de l'atelier d'Arles. À l'aide de ces indices on pourrait supposer que le dépôt de cet exemplaire est intervenu aux alentours du milieu du $\mathrm{IV}^{\mathrm{e}} \mathrm{s}$. après J.-C.

À la lecture de l'inventaire du mobilier contenu dans les sépultures du Trillet, du Pillon et des Girardes, il apparaît que le dépôt monétaire dans lc ritc funćrairc cst loin d'être systématique dans les nécropoles régionales du IV ${ }^{e}$ s. et du début du $V^{e} s$.; d'ailleurs, il ne l'est pas non plus dans les nécropoles de la région Rhône-Alpes datées du Haut-Empire (Collectif, 1987). En revanche, si dans les nécropoles du Haut-Empire on ne recueille qu'une ou deux monnaies par dépôt de crémation ou par tombe, on constate que le nombre de monnaies déposées dans une même sépulture dans les nécropoles de l'Antiquité tardive est parfois important (Feugère $e t$ al., 1987, p. 85). On recense huit monnaies dans la seule 
sépulture SP5008 des Girardes et six unités dans la tombe SP505 de la nécropole du Pillon : ces dernières sont la plupart du temps disposées en pile et parfois dans des récipients.

Par ailleurs, les deux dépôts multiples du Pillon et des Girardes, par leur homogénéité chronologique et par les qualités intrinsèques des monnaies déposées, semblent démontrer que, la plupart du temps, on réserve aux défunts des monnaies fraîchement frappées qui sont issues d'ateliers gaulois: Lyon, Arles ou Trèves. Leur diffusion rapide jusqu'aux alentours de leur lieu d'enfouissement a limité leur temps de circulation. Dans les nécropoles du Haut-Empire, on trouve aussi bien des monnaies fleur de coin que des exemplaires quasiment lisses qui ont très longuement circulé avant d'être dédiés aux morts (Cécillon, 1999).

Par ailleurs, contrairement aux monnaies présentes dans le circuit monétaire du IV ${ }^{c}$ s., on note que les monnaies déposées rituellement dans le cas des nécropoles du Pillon et des Girardes sont en grande partie des espèces de poids lourd. En effet, les niveaux d'occupation de l'Antiquité tardive livrent rarement de monnaies au poids élevé, ces dernières étant thésaurisées par la population (Brénot, 1986).

Si les monnaies ont été choisies ou triées pour leur qualité ou leur poids, l'ont-elles été aussi pour les motifs véhiculés par leurs revers? Le nombre élevé de sept unités sur huit de Magnence et de Décence marquées du chrisme (tombe SP5008 des Girardes) conduit à s'interroger sur le symbolisme de ce dépôt. N'a-t-on pas souhaité formaliser l'adhésion profonde du défunt et/ou de sa famille à la religion chrétienne ? Par le nombre et le symbolisme de ces monnaies, il semble qu'il faille également s'interroger sur la modalité de ce rite du dépôt monétaire - que penser de l'idée de l'obole à Charon qui ne fait pas l'unanimité ? Ne peut-on pas plutôt concevoir dans le geste du dépôt monétaire, qui plus est multiple, une substitution aux dépôts d'objets (bijoux, parures, etc.) en guise d'offrande ou de message symbolique?

Le manque crucial de publication concernant les pratiques des rites funéraires, notamment des caractéristiques des dépôts monétaires dans les nécropoles du Haut-Empire et celles de l'Antiquité tardive, indique clairement que des recherches sur ces sujets doivent être poursuivies, ne serait-ce que sur les plans régionaux.

C. C.

\section{DATATION DES ENSEMBLES FUNÉRAIRES}

La chronologie de chaque ensemble funéraire repose essentiellement sur la typologie des sépultures et sur la datation du mobilier; aucun de ces ensembles ne livre de contexte stratigraphique et seuls quelques squelettes du Trillet à Meyrieu ont fait l'objet d'une datation par le radiocarbone.

Toutes les sépultures du Pillon à Marennes sont pourvues d'un dépôt funéraire. En revanche, les ensembles du Trillet à Meyzieu et des Girardes à Lapalud comportent respectivement vingt et une et neuf sépultures dépourvues de mobilier céramique (soit $66-67 \%$ ). Dans chaque site, celles qui en possèdent sont majoritairement situées dans un secteur (moitić ouest au Trillet, moitić nord aux Girardes). Dans la mesure où la moitié est de l'ensemble du Trillet renferme quelques sépultures orientées différemment des autres (fig. 4), les fouilleurs ont émis l'hypothèse d'une utilisation de longue durée et, dans le but de la démontrer, ont effectué des datations par le radiocarbone sur cinq sépultures (laboratoire de Lyon I) (fig. 25). Les résultats donnent une fourchette extrêmement large, du milieu du III' au milieu du VIII' $s$. ; la seule période de recouvrement est le V $\mathrm{V}^{\mathrm{e}} \mathrm{s}$., sauf pour la sépulture SP9 dont la limite de variation la plus haute est la fin du VIe s. Ces dates ne sont pas en contradiction avec la typologie des sépultures, puisque si les coffres et les coffrages sont représentatifs des IVe-Ve $s$., on les rencontre largement jusqu'au VIII ${ }^{\mathrm{e}}$ s.; par ailleurs, les fosses rupestres en roche meuble sont fréquentes dès la fin du $\mathrm{VII}^{\mathrm{e}} \mathrm{s}$. en contexte rural, et la sépulture SP9, datée par le radiocarbone entre la fin du VI ${ }^{\mathrm{e}}$ et le milieu du VIII ${ }^{\mathrm{e}}$ s., est de ce type. Il est cependant impossible d'attribuer les tombes SP3 et SP5 du Trillet au haut Moyen Âge sur le seul critère de leur typologie, équivalente à celle de la sépulture SP9. En effet, la sépulture SP2, également représentée par une fosse rupestre en roche meuble anthropomorphe, est rattachée par son mobilier à l'Antiquité tardive ( $c f$. inventaire, p. 342), rappelant en cela un groupe de sépultures fouillées à Lunel-Viel. Les tombes SP3 et SP5 étant dépourvues d'attribut mobilier, on ne dispose donc d'aucun critère pour les dater. On note que seules les valeurs obtenues sur le squelette SP8 (cal. 437-625 après J.-C.) sont cohérentes avec la datation des céramiques. Les limites les plus récentes ne correspondent en revanche pas aux datations fournies par le 
mobilier céramique quel qu'il soit, ce qui n'est pas étonnant puisque le dépôt funéraire disparaît dans notre région à la fin du $\mathrm{IV}^{\mathrm{e}} \mathrm{s}$. et que les sépultures " habillées " y sont exceptionnelles. Seule la boucle en fer de la sépulture SP27 peut être attribuée à des contextes allant $\mathrm{du} \mathrm{V}^{\mathrm{e}} \mathrm{au} \mathrm{VII}^{\mathrm{e}} \mathrm{s}$. Mis à part la sépulture SP9, nous n'avons aucun argument pour envisager que les sépultures orientées d'est en ouest sont plus tardives, bien que deux d'entre elles se surimposent aux tombes orientées nordsud. L'intervalle de confiance $d u{ }^{14} \mathrm{C}$ de la sépulture SP29 par exemple est proche de ceux obtenus sur les sépultures SP8 et SP24 orientées nord-sud (fig. 25).

De manière générale, les datations fournies par les monnaies, la poterie et le mobilier métallique sont cohérentes. Cependant, dans la sépulture SP505, le matériel céramique semble un peu plus tardif (dernières décennies du $\mathrm{IV}^{\mathrm{c}} \mathrm{s}$./début du $\mathrm{V}^{\mathrm{c}} \mathrm{s}$.) que la date d'émission des monnaies (première moitié du $\mathrm{IV}^{\mathrm{e}} \mathrm{s}$.). Compte tenu de l'état actuel de nos connaissances des céramiques tardives, ce phénomène n'est pas totalement surprenant. Mais la pratique de la thésaurisation pourrait fournir une explication (Brénot, 1986) ; en effet, si les monnaies sont thésaurisées en groupe, elles peuvent tout à fait être réintroduites de la même manière lors d'un décès...

Ainsi, si les ensembles funéraires du Pillon et des Girardes semblent chronologiquement cohérents (seconde moitié du IV $\mathrm{C}^{\mathrm{s}} \mathrm{s}$ au plus tôt) sur la base de la typologie des sépultures et du mobilier dans le premier cas et par défaut dans le second, celui du Trillet paraît s'étendre sur une plus longue durée. L'examen des dates ${ }^{14} \mathrm{C}$ montre qu'au moins une sépulture (SP9) s'écarte d'un groupe pouvant être daté des $\mathrm{IV}^{\mathrm{e}}$ et $\mathrm{V}^{\mathrm{e}} \mathrm{s}$. par l'ensemble des paramètres (tombes, mobilier, radiocarbone). La variation observée sur les autres dates n'exclut pas, en effet, une datation du $\mathrm{V}^{\mathrm{e}} \mathrm{s}$., l'intervalle de confiance $\mathrm{du}{ }^{14} \mathrm{C}$ ne pouvant pas être confondu avec la durée réelle de l'ensemble funéraire qui peut être plus courte. Par ailleurs, la longue durée d'utilisation des contenants non cloués fait qu'il est impossible d'attribuer les sépultures de ce type à l'Antiquité tardive ou au haut Moyen Âge, lorsqu'elles sont dépourvues de mobilier. La présence de sépultures plus tardives que le noyau principal doit ainsi être évoquée, mais il n'est pas possible de les localiser toutes ni d'en estimer le nombre exact. D'autre part, l'hypothèse d'une datation légèrement postérieure des sépultures tardo-antiques du Trillet

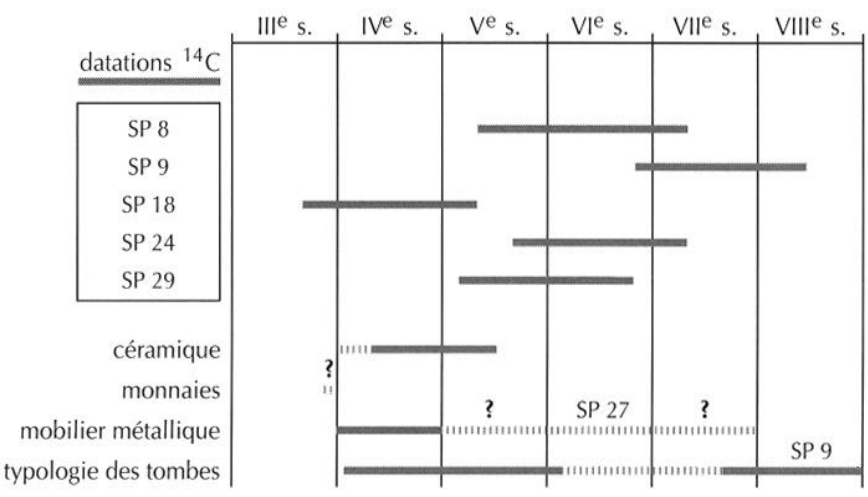

Fig. 25 - Datations des sépultures du Trillet à Meyzieu selon les divers critères utilisés (graphique: D. Castex; DAO: G. Macabéo).

par rapport à celles des deux autres sites peut être envisagée. En effet, on y constate l'absence totale de cercueils cloués, supposés être remplacés totalement par les coffrages dans le courant du $V^{e} s$., tandis qu'une céramique pourrait être attribuée à la fin du IV $\mathrm{s}$./début $\mathrm{V}^{\mathrm{e}}$ s. (SP2, cf. supra).

\section{MODALITÉS DES DÉPÔTS FUNÉRAIRES}

\section{CHOIX ET FRÉQUeNCE dE LA VAISSELLE D'ACCOMPAGNEMENT}

L'étude des dépôts de céramique des trois ensembles funéraires témoigne à la fois de l'homogénéité des choix de formes au sein de l'éventail existant et de la diversité des associations potentielles.

Les cruches de types Des. 84 ou 85 sont un excellent exemple de la grande homogénéité dans les choix des dépôts funéraires. On les retrouve non seulement dans chacun des trois ensembles étudiés, mais également dans les nécropoles contemporaines du sud de la Gaule et dans la plupart des habitats contemporains. Il s'agit en effet de vaisselle à usage domestique fréquemment répertoriée dans les fouilles d'habitat. Cependant, il est clair que l'on a fait un choix au sein du vaisselier et que des associations sélectives ont été pratiquées. On retient prioritairement pour le défunt de la vaisselle fine destinćc habituellement à la table. Elle est majoritaire sur les trois ensembles concernés, avec toutefois des fréquences très variables, une majorité écrasante au Trillet (cinq vases sur six) et une nette majorité au Pillon (dix-neuf vases sur vingtsept), mais la céramique commune retrouve une place notable aux Girardes (cinq communes pour quatre fines). 


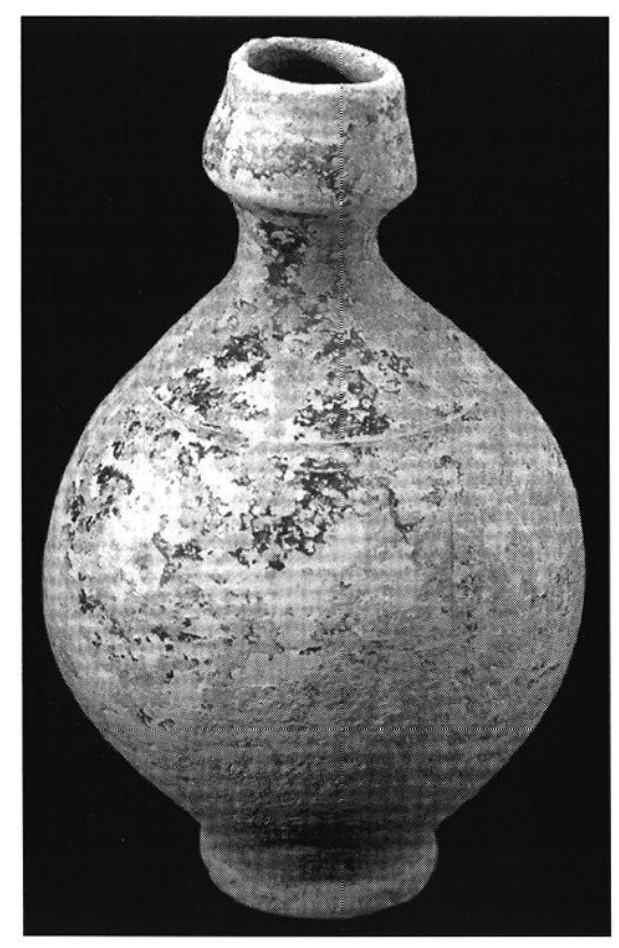

Fig. 26 - Les dépôts céramiques : vase de "second choix "? (photo:J.-M. Degueule, musée de la Civilisation gallo-romaine, Lyon).

La cruche de la tombe SP11 du Trillet, de type Des. 84, constitue un exemplaire particulier dans nos séries. En effet, bien qu'elle se rapproche d'un type connu dans le vaisselier domestique (Des. 85), elle semble appartenir à un lot de " second choix », en témoignent son aspect mal tourné, son revêtement médiocre et surtout l'absence originelle d'anse (fig. 26).

Deux vases sont d'une dimension inférieure à celle connue dans le vaisselier courant: une cruche en céramique à revêtement argileux du Trillet (SP11) et une bouilloire en céramique commune du Pillon (SP515). Des auteurs ont mis en évidence la présence de petits vases qu'ils mettent en relation avec des tombes d'enfants (Bel, 1992, p. 149), mais, dans les deux cas qui nous concernent, ils sont destinés à des adultes.

Au sein de la vaisselle de table, la fonction qui prime est la présentation des liquides dans des gobelets et des cruches. Le Trillet n'a livré que des dépôts uniques constitués de vases à liquides. Au Pillon, les vases à liquides constituent $44 \%$ des dépôts avec une majorité de gobelets. Aux Girardes, le choix s'équilibre entre vases à liquides et vases à solides.
Mais seul l'ensemble funéraire du Pillon permet d'analyser les associations de dépôts sur un nombre significatif de sépultures.

Les sépultures à un dépôt en céramique (tabl. I)

Le choix des vases du Trillet est très spécifique avec un seul dépôt par tombe et uniquement des vases à liquides en céramique fine, avec un doute pour le pot en céramique commune grise de la tombe SP2. On ne peut fixer avec certitude l'usage domestique de ce petit pot avec une anse. Destiné à passer au feu, il pouvait tout aussi bien servir à cuire des aliments solides, semiliquides ou à chauffer des liquides. S'il est destiné à présenter des liquides, nous avons donc systématiquement et uniquement des vases à liquides au Trillet.

Aux Girardes, la sépulture SP5058 comporte un seul gobelet.

Trois sépultures du Pillon ne livrent qu'un dépôt en céramique. Pour deux d'entre elles, l'information n'est pas fiable car la tombe était dans un très mauvais état de conservation. La sépulture SP512 ne fournit qu'une coupe à présenter des aliments solides.

\section{Les sépultures à deux dépôts en céramique (tabl. I)}

Les six sépultures du Pillon à deux dépôts comprennent dans cinq cas un gobelet à liquides et un plat ou une coupe à solides. Dans le sixième cas, il s'agit d'un gobelet associé à une coupelle. On a vu précédemment que la fonction des coupelles n'était pas fixée : vases à liquides ou à solides. Si on classe cette coupelle dans les vases à présenter les offrandes de nourriture solide, on obtient le schéma majoritaire : vase à solides accompagnant un vase à liquides. Dans ces duos de dépôts, le vase à liquides est toujours un gobelet, sauf dans la sépulture SP515 où il s'agit d'une bouilloire en céramique commune. Le vase à aliments solides peut être une assiette en céramique fine ou une coupe, ou un plat en céramique commune.

On observe les mêmes associations aux Girardes: coupe en céramique commune et cruche en céramique fine dans les tombe SP5008 et SP5038, cruche ou gobelet en céramique fine et plat en céramique commune dans la tombe SP5023. De manière originale, les dépôts de la sépulture SP5001, un plat en céramique commune et un tonnelet-cruche à pâte calcaire, entérinent cette association, vase à liquides et vase à solides. 
Les sépultures à trois dépôts en céramique (tabl. I)

Le Pillon a livré quatre sépultures contenant trois vases.

Les sépultures SP504 et SP508 offrent un schéma commun : une cruche, un gobelet et un plat, soit deux vases à liquides et un vase à aliments solides.

Les sépultures SP505 et SP514 ont en commun un pot en céramique commune grise, mais la SP514 comprend un plat et une coupelle, alors que la SP505 a livré une coupe et un gobelet.

Aux Girardes, seule la tombe SP5002 a fourni trois dépôts : une cruche en céramique fine, une coupe et un pot en céramique commune.

En conclusion, on remarque que lorsqu'un seul vase a été déposé il s'agit généralement d'un vase à liquides et le plus souvent d'un gobelet. Lorsque deux vases ont été déposés, on a pris soin d'associer un vase à liquides et un vase à solides, le gobelet reste alors le représentant majoritaire du vase à liquides. C'est dans les sépultures à deux dépôts que le schéma est le plus strict : elles contiennent systématiquement un représentant des vases à liquides et un des vases à solides, comme s'il s'agissait d'un minimum pour l'éternité.

Dans les sépultures à trois dépôts, bien que le schéma de base solides/liquides semble généralement respecté, le troisième dépôt peut, soit doubler les liquides comme dans la tombe SP508 du Pillon où on a une cruche et un gobelet associés à un plat, soit doubler les solides comme dans la tombe SP505 où un pot et une coupe sont associés à un gobelet. Aux Girardes, dans la tombe SP5002, une coupe et un pot en céramique commune accompagnent une cruche en céramique fine.

\section{Comparaisons régionales et extra-régionales}

Les comparaisons quantitatives sont délicates. À Lansargues, il y a deux à cinq dépôts par tombe ; à Laudun, leur nombre varie de un à sept avec une nette dominante des dépôts à trois et cinq vases ; quai Arloing, il varie de un à six. L'ensemble funéraire du Trillet se détache nettement de ce cadre ; en effet, nous n'avons trouvé aucun exemple de nécropole où toutes les tombes ne comportent qu'un seul dépôt comme dans ce site.

L'omniprésence et la suprématie des vases à liquides, cruches ou gobelets en céramique à revêtement argileux, sont manifestes dans de nombreuses nécropoles contemporaines dans la basse vallée du Rhône (Gagnière, 1965, p. 65), à Laudun (Feugère et al., 1987, p. 89), La Côte ou quai Arloing (Tranoy, 1995, p. 810). Les formes présentes sont généralement très proches ; le gobelet Lam. 14/26 et les cruches Des. 84 et 85 sont attestés dans la plupart des nécropoles du sud de la Gaule (Girard, Raynaud, 1982, p. 166).

Les principaux schémas d'associations de formes sont également en vigueur dans d'autres nécropoles tardives, dont celle de Lansargues dans l'Hérault (Girard, Raynaud, 1982) et dans les nécropoles de la basse vallée du Rhône (Gagnière, 1965, p. 67) ${ }^{2}$. Ici, chaque tombe avec dépôt contient au moins un vase à liquides et un vase à solides. Lorsque le nombre de dépôts est supérieur à deux, sont associés soit d'autres vases à liquides, soit d'autres vases à solides sans règles particulières.

\section{REPRÉSENTATIVITÉ DES VASES}

Seuls dix vases sur quarante-cinq sont complets, dont cinq intacts (tabl. I et II). Les fragments manquants n'ayant jamais été déposés dans les tombes, la plupart des dépôts sont donc mutilés. Les mutilations concernent aussi bien la vaisselle de table que la céramique culinaire, les vases à liquides que les vases à solides. Pour les cruches, gobelets, coupes, coupelles et pots, ces mutilations se situent uniquement sur la partie supérieure des vases. En ce qui concerne les plats, des fragments de fonds peuvent également manquer.

Les taux de représentation des vases mutilés oscillent entre 30 et $98 \%$ (tabl. I). Dans le cas du taux de représentativité le plus faible, n'est conservé que le fond du vase tronqué à mi-panse; dans le cas de la plus faible mutilation, il manque seulement un fragment de lèvre en forme de " $V$ ". Cette petite cassure très particulière est présentc sur ncuf vascs dont scpt nc sont affectés d'aucune autre mutilation (fig. 27). On la retrouve sur les pots en céramique commune grise du Trillet (SP2), des Girardes (SP5002) et du Pillon (SP514), sur une cruche à revêtement argileux des Girardes (SP5002) (fig. 28), sur les gobelets du Pillon (SP505, SP511), sur une coupelle du Pillon (SP514) et sur un plat du Pillon (SP510). L'aspect de cette cassure nous incite à penser qu'un outil

2. L'absence de catalogues dans de nombreuses publications limite malheureusement les comparaisons de types fonctionnels. 
Tabl. I - Tableau récapitulatif des dépôts céramiques (réalisation : C. Bonnel).

\begin{tabular}{|c|c|c|c|c|c|c|c|c|c|c|}
\hline Site & Sép. & no objet & Type de prod. & Identification & Fonction & $\%$ conservé & Partie absente & Diam. inf. & Diam. sup. & $\mathrm{H}$ \\
\hline \multirow[t]{6}{*}{ Le Trillet } & 1 & 1 & revêt. argileux & cruche - proche Des. 84 & à liquides & sauf anse & anse & 50 & 22 & 147 \\
\hline & 2 & 1 & commune grise & pot ansé & à solides & $98 \%$ & fragment de bord & 44 & 80 & 117 \\
\hline & 10 & 1 & revêt. argileux & gobelet - P. 62 var 130/Lam. 14/26 & à liquides & $80 \%$ & $3 / 4$ du col & 42 & 65 & 125 \\
\hline & 11 & 1 & revêt. argileux & petite cruche - Portout lèvre $E$ & à liquides & sauf anse & anse & 34 & 33 & 90 \\
\hline & 15 & 1 & revêt. argileux & cruche - Des. $85 /$ P. 95 & à liquides & complet & & 49 & 36 & 156 \\
\hline & 16 & 2 & revêt. argileux & gobelet - P. 62/Lam. 14/26 & à liquides & complet & & 40 & 56 & 110 \\
\hline \multirow[t]{27}{*}{ Le Pillon } & 504 & 1 & revêt. argileux & plat - P. 1 & à solides & $50 \%$ & fragments panse, bord, fond & 188 & 250 & 41 \\
\hline & & 2 & revêt. argileux & gobelet - P. 66 & à liquides & $60 \%$ & fragments panse, bord & 34 & 51 & 14 \\
\hline & & & revêt. argileux & cruche non identifiée & à liquides & $30 \%$ & fragments panse, bord, col & 37 & 19 & n. i. \\
\hline & 505 & 2 & commune rotge & coupe carénée & à solides & complet & & 49 & 145 & 55 \\
\hline & & 3 & commune grise & pot à lèvre en amande & à solides & $90 \%$ & fragments de panse & 55 & 85 & 112 \\
\hline & & 1 & revêt. argileux & gobelet - P. 76 & à liquides & $98 \%$ & fragment de bord & 28 & 53 & 117 \\
\hline & 506 & 1 & revêt. argileux & coupe - P. var. 24 & à solides & $80 \%$ & fragments de panse & 72 & 167 & 59 \\
\hline & & 2 & revêt. argileux & gobelet ou cruche & à liquides & $30 \%$ & col et $1 / 2$ panse & 40 & n. i. & n. i. \\
\hline & 507 & 1 & revêt. argileux & gobelet - P. 66 & à liquides & complet & & 28 & 48 & 98 \\
\hline & & 2 & revêt. argileux & plat - P. 1 & à solides & complet & & 127 & 219 & 45 \\
\hline & 508 & 1 & revêt. argileux & cruche non identifiée & à liquides & $50 \%$ & col et $1 / 2$ panse & 52 & n. i. & n. i. \\
\hline & & 2 & revêt. argileux & gobelet - P. 65 & à liquides & $90 \%$ & fragment de panse & 28 & 50 & 108 \\
\hline & & 3 & commune grise & plat & à solides & $80 \%$ & frgt bord et panse + un trou & 196 & 240 & 65 \\
\hline & 509 & 1 & revêt. argileux & gobelet - P. 66 & à liquides & $80 / 90 \%$ & anse et $3 / 4 \mathrm{col}$ & 27 & 46 & 97 \\
\hline & & 2 & revêt. argileux & plat - Lam. 4/36-P. 7 & à solides & $99 \%$ & fragment de lèvre & 67 & 194 & 38 \\
\hline & 510 & 1 & revêt. argileux & gobelet - Lam. 14/26 & à liquides & $40 \%$ & $1 / 2$ panse et $3 / 4$ lèvre & 34 & n. i. & n. i. \\
\hline & 511 & 1 & revêt. argileux & gobelet - P. 66 & à liquides & $95 \%$ & fragment de lèvre & 32 & 56 & 87 \\
\hline & & 2 & commune non id. & plat à lèvre éversée & à solides & $60 \%$ & frgts panses, bord, fond & 135 & 165 & 33 \\
\hline & 512 & 1 & revêt. argileux & plat - P. 20 & à solides & $98 \%$ & fragment de lèvre & 66 & 170 & 40 \\
\hline & 513 & 1 & revêt. argileux & gobelet - P. 75 & à liquides & $70 \%$ & fragments panse, bord, anse & 37 & 54 & 110 \\
\hline & & 2 & revêt. argileux & petite coupe - P. 30 & à solides & complet & & 48 & 102 & 50 \\
\hline & 514 & 3 & revêt. argileux & petite coupe - P. 33 & à solides & $98 \%$ & fragment de bord & 35 & 83 & 36 \\
\hline & & 1 & commune grise & plat à panse oblique & à solides & complet & & 123 & 200 & 60 \\
\hline & & 2 & commune grise & pot à lèvre en bourrelet & à solides & $98 \%$ & fragment de lèvre & 39 & 80 & 103 \\
\hline & 515 & 2 & sigillée africaine $\mathrm{C}$ & plat - Hayes $50 \mathrm{~b}$ & à solides & complet & & 152 & 298 & 49 \\
\hline & & 1 & commune rouge & cruche bec pincé & à liquides & complet & & 49 & 46 & 135 \\
\hline & 516 & 1 & commune rouge & plat à lèvre en bourrelet & à solides & $20 \%$ & fond et $2 / 3$ panse & 135 & 155 & 44 \\
\hline \multirow[t]{12}{*}{ Les Girardes } & \begin{tabular}{|l|}
5001 \\
\end{tabular} & 1 & pâte calcaire & tonnelet & à liquides & $80 \%$ & $\mathrm{col}$ & diam. 90 & n. i. & n. i. \\
\hline & & & commune grise & plat & à solides & $70 \%$ & fragments fond, bord & n. i. & n. i. & n. i. \\
\hline & 5002 & 1 & revêt. argileux & cruche - Des. 84 & à liquides & $70 \%$ & $1 / 3$ panse et 1 frgt lèvre & 45 & 32 & 145 \\
\hline & & 2 & commune rouge & jatte/marmite carénée & à solides & $80 \%$ & $1 / 4$ panse et col & 55 & 121 & 43 \\
\hline & & 3 & commune grise kao. & pot - Bonnet fig. $12 \mathrm{n}^{\circ} 5$ & à solides & comolet & & 58 & 104 & 120 \\
\hline & 5008 & 2 & commune rouge & jatte/mar. - Bonnet fig. $15 n^{\circ} 10$ & à solides & $70 \%$ & fragments bord, panse & 75 & 160 & 72 \\
\hline & & 1 & revêt. arg. ou clair & cruche non identifiée & à liquides & $60 \%$ & col, anse, frgts de panse & 39 & n. i. & n. i. \\
\hline & 5023 & 2 & revêt. argileux & plat - Clair-b 102 & à solides & $90 \%$ & $1 / 2$ bord & 155 & 230 & 45 \\
\hline & & 1 & revêt. argileux & cruche, gobelet? & \begin{tabular}{|l|} 
à liquides \\
\end{tabular} & $80 \%$ & $\mathrm{col}$ & 45 & n. i. & n. i. \\
\hline & 5038 & 1 & revêt. argileux & cruche - Clair-b 89 & à liquides & $70 \%$ & fragments panse et col & 49 & n. i. & n. i. \\
\hline & & 2 & commune? & jatte non identifiée & à solides & $90 \%$ & bord & 42 & n. i. & n. i. \\
\hline & 5058 & 1 & revêt. argileux & gobelet non identifié & à liquides & trop fragmenté & bord & $?$ & $?$ & $?$ \\
\hline
\end{tabular}

de type pince a pu être utilisć et qu'elle a donc été volontairement pratiquéc. Il parait en effet peu probable qu'un bris dû à l'usage dans le cadre domestique ait pu provoquer une cassure aussi anguleuse, nette et caractéristique, sur des catégories de céramiques dont les épaisseurs de parois, la solidité et les fonctions sont si diverses.

Dans cinq cas, toute la partie supérieure du vase manque comme si elle avait été décolletée. Il s'agit de cruches du Pillon (SP504, SP508) et des Girardes (SP5008, SP5023), d'une forme ouverte en céramique commune des Girardes (SP5038) et d'un gobelet des Girardes (SP5058).
L'anse est souvent absente, aussi bien lorsque le col ou une partie du col manquent, que sur une cruche de petit module par ailleurs complète au Trillet (SP11).

Les fonds sont toujours complets. Néanmoins, les lacunes sur la panse sont aussi fréquentes (dix cas dont deux perforations). Deux vases du Pillon présentent des perforations faites après cuisson par un outil fin et pointu (fig. 29). La cruche en céramique commune de la sépulture SP515 est perforée sur la partie supéricure de la panse légèrement au-dessus de la carc̀ne. Cette petite perforation rectangulaire de $3 \mathrm{~mm}$ sur $5 \mathrm{~mm}$ perce de biais la panse du vase, sa finesse pourrait indiquer qu'elle a été faite à l'aide d'un clou ou d'un outil de type 
Tabl. II - Tableau récapilulatif des types de bris et de cassures des céramiques et situation par sépultures (réalisation : F: Blaizot et C. Bonnel).

\begin{tabular}{|c|c|c|c|c|c|c|c|c|c|c|}
\hline Site & Sép. & nobjet & complet intact & complet fragmenté & mutilé non fragmenté & mutilé fragmenté & fgts hors contenant & tête & pieds & membre inf. \\
\hline \multirow[t]{6}{*}{ Le Trillet } & 1 & 1 & & & cruche & & & G & & \\
\hline & 2 & 1 & & & pot & & & & & genou D \\
\hline & 10 & 1 & & & gobelet & & & $\mathrm{D}$ & & \\
\hline & 11 & 1 & & & cruche & & & & $\mathrm{D}$ & \\
\hline & 15 & 1 & cruche & & & & & & & jambe D \\
\hline & 16 & 2 & gobelet & & & & & D & & \\
\hline \multirow[t]{27}{*}{\begin{tabular}{|l|} 
Le Pillon \\
\end{tabular}} & 504 & 1 & & & & cruche & & & & lat. G \\
\hline & & 2 & & & & gobelet & & & & lat. G \\
\hline & & & & & & plat & & & & lat. G \\
\hline & \begin{tabular}{|l|}
505 \\
\end{tabular} & 2 & & & & gobelet & $x$ & & $x$ & \\
\hline & & 3 & & coupe & & & & & & $x$ \\
\hline & & 1 & & & pot & & & & D & \\
\hline & \begin{tabular}{|l|}
506 \\
\end{tabular} & 1 & & & cruche/gobelet & & & $\mathrm{D}$ & & \\
\hline & & 2 & & & coupe & & & & $x$ & \\
\hline & \begin{tabular}{|l|}
507 \\
\end{tabular} & 1 & gobelet & & & & & & $x$ & \\
\hline & & 2 & & plat & & & & & $x$ & \\
\hline & \begin{tabular}{|l|}
508 \\
\end{tabular} & 1 & & & & cruche & $x$ & & $x$ & \\
\hline & & 2 & & & gobelet & & & $x$ & & \\
\hline & & 3 & & & & plat & $x$ & & $x$ & \\
\hline & \begin{tabular}{|l|}
509 \\
\end{tabular} & 1 & & & gobelet & & & $\mathrm{D}$ & & \\
\hline & & 2 & & & & plat & $x$ & $?$ & $?$ & $?$ \\
\hline & \begin{tabular}{|l|}
510 \\
\end{tabular} & 1 & & & gobelet & & & $\mathrm{G}$ & & \\
\hline & \begin{tabular}{|l|}
511 \\
\end{tabular} & 1 & & & & gobelet & & & $x$ & \\
\hline & & 2 & & & plat & & & & $x$ & \\
\hline & \begin{tabular}{|l|}
512 \\
\end{tabular} & 1 & & & plat & & & & $x$ & \\
\hline & \begin{tabular}{|l|}
513 \\
\end{tabular} & 1 & & & gobelet & & & & $x$ & \\
\hline & & 2 & coupelle & & & & & & $x$ & \\
\hline & \begin{tabular}{|l|}
514 \\
\end{tabular} & 3 & & & coupelle & & & & $x$ & \\
\hline & & 1 & & & plat & & & & $x$ & \\
\hline & & 2 & & & pot & & & & $x$ & \\
\hline & \begin{tabular}{|l|}
515 \\
\end{tabular} & 2 & & & cruche & & & G & & \\
\hline & & 1 & & plat & & & & & $x$ & \\
\hline & 516 & 1 & $?$ & $?$ & $?$ & $?$ & & & $x$ & \\
\hline \multirow{12}{*}{ Les Girardes } & 5001 & 1 & & & tonnelet & & & & $x$ & \\
\hline & & & & & plat & & & & $x$ & \\
\hline & 5002 & 1 & & & cruche & & & & & lat. G \\
\hline & & 2 & coupe & & & & & & & lat. D \\
\hline & & 3 & & & pot & & & & & lat. G \\
\hline & \begin{tabular}{|l|}
5008 \\
\end{tabular} & 2 & & & cruche & & & & $?$ & \\
\hline & & 1 & & & coupe & & & & $?$ & \\
\hline & 5023 & 2 & & & cruche & & & & & lat. genou D \\
\hline & & 1 & & & plat & & & & & lat. pied D \\
\hline & \begin{tabular}{|l|}
5038 \\
\end{tabular} & 1 & & & cruche & & & $?$ & $?$ & $?$ \\
\hline & & 2 & & & coupe & & & $?$ & $?$ & $?$ \\
\hline & \begin{tabular}{|l|}
5058 \\
\end{tabular} & 1 & & & gobelet & & & & & \\
\hline
\end{tabular}

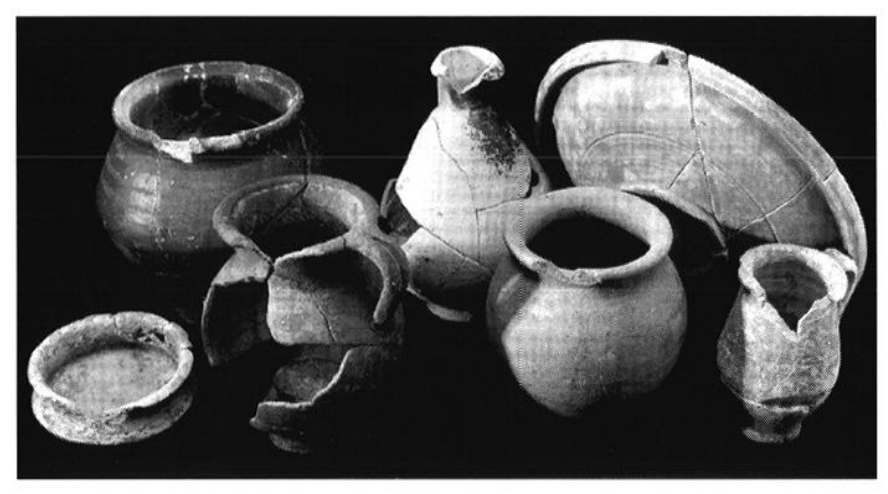

Fig. 27 - Les dépôts céramiques : cassures en " $V$ » et bris (photo :J.-M. Degueule, musée de la Civilisation gallo romaine, Lyon).

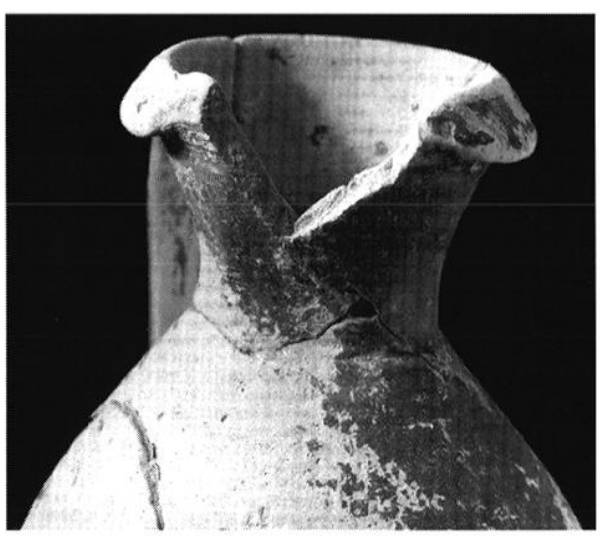

Fig. 28 - Les dépôts céramiques : délail de la cruche de la sépulture SP5002 des Girardes à Lapalud (cf. pl. VI, $n^{\circ} 1$ ) (photo :

J.-M. Degueule, musée de la Civilisation gallo-romaine, Lyon). 


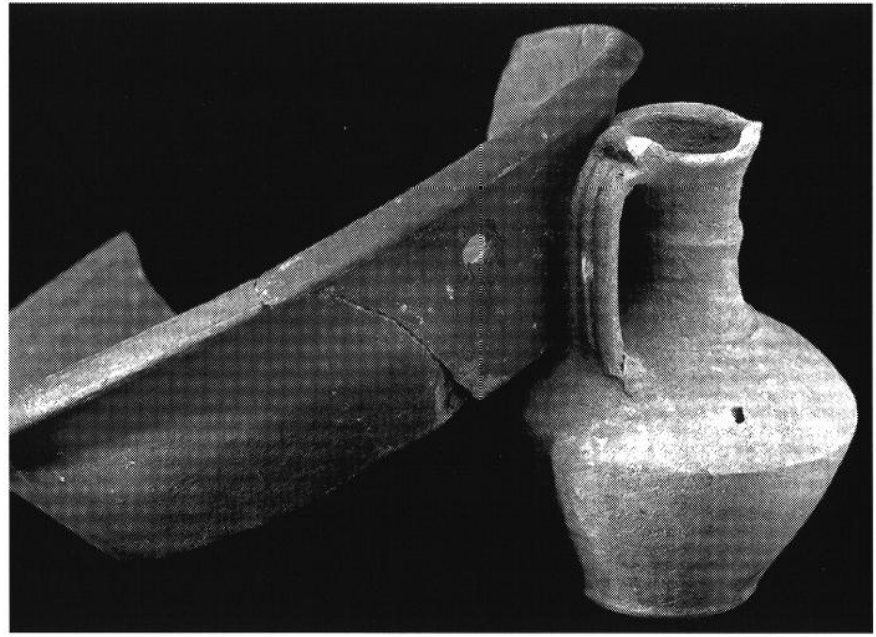

Fig. 29 - Les dépôts céramiques : vases à perforation après cuisson (photo: J.-M. Degueule, musée de la Civilisation gallo-romaine, Lyon).

foret ${ }^{3}$. Le deuxième exemple de perforation a été observé sur un plat en céramique commune (SP508). Un trou circulaire de $8 \mathrm{~mm}$ de diamètre a été fait légèrement au-dessus du milieu de la panse à $10 \mathrm{~mm}$ sous la lèvre. On voit très nettement que cette tâche a été effectuée à l'aide d'un outil et de l'extérieur du vase, comme en témoignent les éclats autour de l'orifice. Dans la mesure où les perforations affectent généralement la panse, elles ne peuvent pas avoir été pratiquées pour suspendre le vase, puisqu'un orifice à ce niveau les aurait rendues inutilisables au quotidien. L'hypothèse d'une réparation est également douteuse, puisque le vase ne présente aucune cassure à cet endroit précis et qu'on ne relève qu'un seul trou. On note qu'il existe en contexte funéraire de nombreuses références à des vases caractérisés par un trou pratiqué après la cuisson (site de Infirmary Field à Chester, $c f$. Crinsell, 1961, p. 488) et, dans certains cas, l'outil utilisé, un clou, a été retrouvé en place (Bérard, 1963, p. 302-306 ; Desaye, 1996 ; Jospin, 1998-1999, p. 24-28).

Les autres mutilations consistent en diverses cassures sans formes particulières, sur les bords, les cols et les panses, qu'il n'est pas possible de systématiser.

3. Des clous à section quadrangulaire perforant des vases ont été découverts dans des tombes à Neuvic en Dordogne et dans l'Yonne (Jospin, 1998-1999, p. 25) et à Argentomagus dans l'Indre (Allain et al., 1992, p. 129).

\section{LA SITUATION DU MOBILIER DANS LA TOMBE}

\section{Le mobilier céramique}

Sur le site du Trillet, les vases sont déposés à l'intérieur du cercueil ou du coffrage de bois qui contient les restes humains. C'est également le cas aux Girardes, tandis qu'au Pillon, les modalités de dépôt du mobilier paraissent plus complexes.

Dans quelques cas, il est possible d'affirmer que les récipients ont été placés dans le contenant du corps, généralement aux pieds et à la tête, en témoignent leur situation, debout sur le fond de la fosse et leur organisation par rapport au squelette (tabl. II) ; ces vases offrent des effets de délimitation linéaire avec les os du squelette, illustrant les limites du contenant (par ex. sur le côté gauche du squelette SP515, fig. 16).

Certains récipients des tombes SP505, SP507, SP508, SP509, SP511 et SP515 sont fragmentés sur place (tabl. II). Dans trois d'entre elles, les morceaux sont répartis à l'intérieur du contenant supposé (SP507, SP511, SP515), mais dans les sépultures SP505, SP508 et SP509 des éléments appartenant aux vases sortent des limites restituées du contenant (fig. 9, 16, 18, 17, 8). Dans la fosse SP508 par exemple, les fragments nos 2 et 13 de la cruche sont dispersés à distance en dehors des parois du contenant, déterminées par l'alignement des os et des autres fragments de céramique ; ce n'est en revanche pas le cas des morceaux de l'assiette (fig. 17). Dans la sépulture SP509, les fragments de l'assiette sont dispersés sur une amplitude de $70 \mathrm{~cm}$ (fig. 8) ; le tesson $\mathrm{n}^{\circ} 1$ situé en amont du crâne se trouve au-delà du clou est, qui marque la limite du contenant, et il repose, ainsi que les fragments $\mathrm{n}^{\text {os }} 4$ et 5 , environ $10 \mathrm{~cm}$ moins profondément que les ossements.

Les céramiques fragmentées, dont les morceaux se situent à l'intérieur des limites du coffre, peuvent avoir reposé à l'origine dans ce dernier et s'être fracturées lors de l'effondrement du couvercle. Mais l'hypothèse qu'elles aient été déposées ainsi brisées doit également être considérée ; par exemple, le gobelet de la fosse SP511 est cassé à mi-panse et la cassure est nette, ce qui paraît curieux pour une fracture occasionnée accidentellement. Il n'est pas exclu non plus que ces céramiques aient été à l'origine déposées sur le couvercle du contenant et qu'elles soient tombées à l'intérieur lors de son pourrissement. L'exemple de l'assiette de la sépulture SP507, qui 
recouvre un gobelet couché, illustrerait ce cas de figure, puisque l'un de ses morceaux est placé de chant contre l'embouchure du gobelet ; cette position semble indiquer qu'il s'est effondré depuis un niveau plus élevé à l'origine. Les fragments disloqués des céramiques des autres tombes reposent sur le fond de la fosse et ne présentent que des pendages faibles, de 3 à $5 \mathrm{~cm}$, n'offrant ainsi pas d'arguments supplémentaires en ce sens.

L'hypothèse d'un dépôt pratiqué à l'origine sur le couvercle pourrait être également valable pour les céramiques des sépultures SP505, SP508 et SP509, dont quelques fragments, qui se trouvent sur le sol de la fosse, sont situés à l'extérieur des limites du contenant. Mais il faudrait, dans ce cas, envisager que l'architecture aménageait un double espace vide, l'un pour le corps (le coffrage ou le cercueil) et l'autre pour la fosse, ce qu'aucun élément ne permet de démontrer dans nos exemples. Il apparaît donc plus plausible que les céramiques de ces trois tombes aient été cassées avant de remblayer la fosse et qu'elles furent rejetées soit dans le contenant encore ouvert, soit sur son couvercle, tandis que quelques fragments se sont dispersés de part et d'autre. De telles pratiques ont ponctuellement été relevées en contexte archéologique, comme sur le site de Saint-Jacques à Paris, où des vases ont été ainsi brisés contre les cercueils (Bourgeois, 1986, p. 293).

La proposition que certaines céramiques aient été brisées au moment des funérailles peut-elle être mise en parallèle avec les lacunes constatées sur quelques récipients (cf. supra) ? Dans ce cas, il s'agirait là de deux représentations différentes d'un bris symbolique, à la suite duquel tous les fragments ne seraient pas systématiquement placés dans la tombe. Mais il est aussi vraisemblable que ces deux formes de bris appartiennent en réalité à deux registres bien distincts, d'autant que les fragments absents semblent avoir été prélevés à l'aide d'un « outil "; dans cette optique, l'un se rapporterait à l'économie funéraire propre au défunt (destruction symbolique des biens du mort par exemple) et l'autre participerait au rituel des vivants (destruction de la vaisselle ayant servi lors des funérailles).

On remarque que les objets fragmentés en place sont systématiquement situés aux pieds ou au niveau des membres inférieurs des squelettes ${ }^{4}$. Les récipients

4. Le cas du gobelet de la sépulture SP508 (fig. 17), dont les fragments s'échelonnent le long du membre supérieur gauche $\left(\mathrm{n}^{\mathrm{os}} 58\right.$ à 61$)$, est complets, comme les vases lacunaires mais non fragmentés, sont indifféremment placés aux pieds (dix vases pour sept tombes) ou à la tête des défunts (cinq tombes). Mais, en réalité, le modèle est différent si l'on examine leur distribution dans l'espace de l'ensemble funéraire. On rencontre en effet les récipients fragmentés en place dans toutes les sépultures situées dans la partie est (SP505, SP507, SP508, SP509 et SP515) et dans une seule de celles qui sont réunies à l'ouest (SP511). Dans le groupe est, les vases qui accompagnent les immatures sont toujours placés aux pieds, alors que pour les adultes le récipient retrouvé non brisé dans le contenant est situé à la tête et les céramiques fragmentées se trouvent aux pieds. Dans le groupe ouest (SP506, SP510, SP511, SP512, SP513, SP514, SP515), les vases sont aux pieds des adultes et placés indifféremment aux pieds ou à la tête chez les immatures. La petite taille de l'échantillon ne permet toutefois pas d'affirmer que ces différences de traitement entre les individus adultes et immatures et entre les groupes sont significatives.

Un cas particulier est représenté dans la sépulture SP504; le mobilier situé latéralement, côté gauche du squelette, se compose de trois céramiques $\left(\mathrm{n}^{\mathrm{os}} 1,2,50\right)$, d'une lampe $\left(\mathrm{n}^{\circ} 8\right)$, d'éléments de parure et d'un dépôt alimentaire ( $\left.n^{\circ} 90\right)$ (fig. 30). L'organisation de ces objets offre deux effets de délimitation, l'un à l'ouest qui passe par le conglomérat d'anneaux circulaires $\left(n^{\circ} 56\right)$ et les fragments de la coupe $\left(n^{\circ} 1\right)$, et l'autre à l'est, défini par l'alignement de la boucle $\left(n^{\circ} 13\right)$, des os les plus à l'ouest du volatile ( $\mathrm{n}^{\text {os }} 33$ et 35 ), d'un tesson appartenant au gobelet $\left(n^{\circ} 102\right)$ et de la cruche $\left(n^{\circ} 2\right)$. Ils semblent ainsi avoir été déposés dans un coffre de forme rectangulaire, placé contre le contenant du corps.

Enfin, dispersés sur le fond de la fosse de la sépulture SP512 du Pillon, se trouvent les restes de deux amphores. Malgré leur situation en fond de fosse, nous les avons interprétés comme du matériel résiduel, chaque vase n'étant représenté que par quelques fragments de panse.

Nous tenterons maintenant de mettre en relation le choix d'un type de vase et sa localisation auprès du défunt. Seuls les dépôts autour du crâne sont très carac-

différent. On suppose qu'il était placé initialement sur l'élément qui surélevait la tête du cadavre, dont l'effondrement aurait produit cette fragmentation. La situation originelle de l'assiette de la sépulture SP509, dont les fragments $\left(\mathrm{n}^{\mathrm{os}} 1,4,5,20,21,22\right)$ sont beaucoup trop dispersés, est inconnue (fig. 8). 
térisés ; il s'agit uniquement de vases à liquides (huit cas) et généralement de gobelets (six cas sur huit). Ces vases à boire sont déposés dans le contenant à côté de la tête du défunt indifféremment à droite ou à gauche, qu'il s'agisse d'un homme, d'une femme ou d'un immature. $\dot{A}$ Lansargues également, tous les vases déposés autour du crâne sont des vases à liquides: cruches ou gobelets (Girard, Raynaud, 1982).

Cela ne signifie pas pour autant que les vases à boire soient exclusivement installés autour de la tête, on les

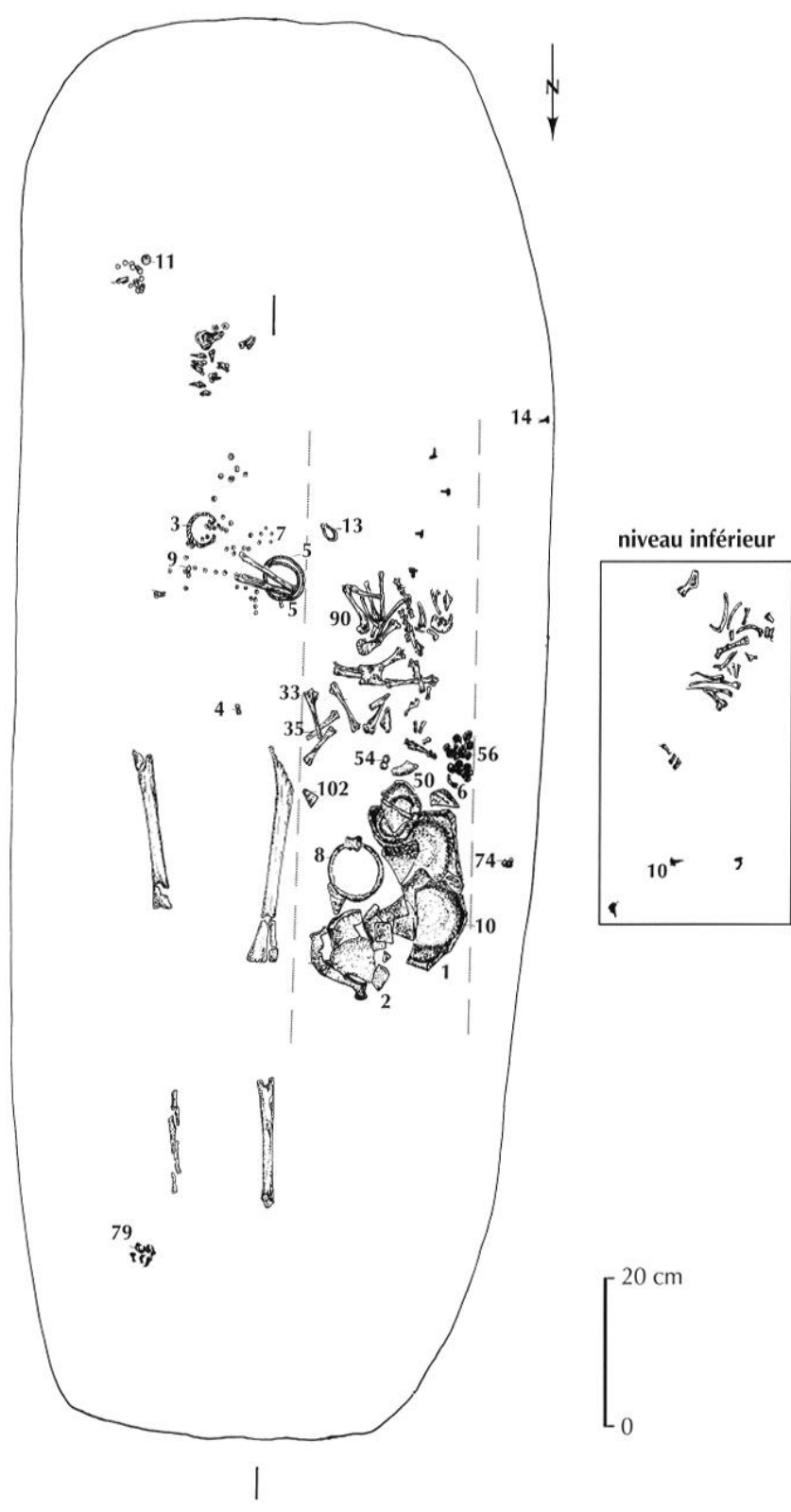

Fig. 30 - Le Pillon à Marennes, sépullure SP504 (cf. pl. II). Ln pointillé: effet de délimitation permettant de restituer le coffret (relevé et dessin : F. Blaizot; DAO: (.. Macabéo). retrouve régulièrement aux pieds des défunts: une cruche aux pieds des squelettes SP11 du Trillet et SP508 du Pillon, des gobelets aux pieds des squelettes SP507 et SP511 du Pillon, et SP5058 des Girardes. En fait, tous les types de vases peuvent être installés aux pieds ou latéralement près des membres inférieurs: cruche, gobelet, coupe, coupelle, plat, pot.

Les vases cassés sur le contenant ne sont pas caractérisés ; il peut s'agir d'assiettes, de cruches ou de gobelets.

\section{Le mobilier métallique}

Les objets métalliques se divisent en plusieurs catégories : les monnaies, les éléments de parure ou les accessoires vestimentaires et les récipients.

La position des monnaies dans les tombes n'est pas systématisée. Toutes semblent avoir été placées dans le contenant, mais pas forcément au contact du corps: deux s'alignent latéralement et à distance des membres inférieurs droits du squelette dans la sépulture SP505 et celle qui accompagne le squelette SP512 se trouve placée latéralement à la cheville gauche. Cependant, dans trois exemples, les monnaies sont au contact des os, comme si elles avaient été placées sur lc corps, ou dans la poche d'un vêtement (au Trillet: SP2, sous la tête fémorale gauche; SP30, dans le tiers supérieur de l'hémi-thorax gauche; au Pillon : SP513, en amont du fémur gauche). Au Pillon, les monnaies de la sépulture SP505 sont empilées dans un pot; elles sont également empilées dans la sépulture SP5008 des Girardes, mais sur le fond de la fosse ; quatre sont situées à côté des céramiques et trois dans ce qui nous semble être le centre de la fosse, sans qu'il soit possible de préciser à quel niveau par rapport au squelette, compte tenu de la destruction des ossements. Une monnaie a été retrouvée parmi les os disloqués de la base du cou du squelette SP5002 des Girardes ; la tête ayant reposé à l'origine sur un coussin céphalique, il est possible que la monnaie ait été déposée dans la bouche du défunt, comme on le rencontre parfois. La présence de monnaies dans les sépultures antiques est fréquente; elle est invariablement mise en relation avec le mythe de l'obole à Charon alors qu'il s'agit d'une interprétation tardive du phénomène (Borza, 1955, p. 142). En réalité, la monnaie, en tant qu'objet d'échange, vient plus probablement se substituer ou compléter le cortège mobilier habituel, en témoigne le fait que l'on n'en trouve pas dans toutes les 
sépultures d'un même ensemble funéraire, comme c'est le cas dans nos séries. On notera que les monnaies n'accompagnent que les individus immatures et les adultes féminins (sachant tout de même que trois adultes qui en possédent sont de sexe indéterminé).

Il semble que la plupart des objets de parure, notamment les bracelets, ont été retrouvés dans des situations cohérentes avec leur emplacement habituel sur le corps : il s'agit alors d'éléments réellement portés par le défunt, ou placés dans leur position d'utilisation; les dépôts effectués dans d'autres endroits de la sépulture restent peu fréquents.

La parure n'est pas toujours portée; au Pillon, les bracelets sont en place autour des avant-bras, mais dans la sépulture SP2 du Trillet, les armilles se trouvent au niveau de la poitrine gauche. Dans la sépulture SP5023 des Girardes, les armilles sont en avant de la diaphyse fémorale gauche, mais les membres supérieurs n'étant pas conservés, on ne peut dire si elles étaient portées. Ces bracelets sont présents dans les sépultures d'enfants et dans deux sépultures d'adultes de sexe indéterminé. Néanmoins, ces objets sont généralement associés aux individus féminins dans les sépultures de la seconde moitié du IVes. et au Ve $\mathrm{s}$. Mis à part les bracelets, les éléments de parure qui accompagnent l'enfant de la sépulture SP504 du Pillon ont été placés dans le coffret avec les céramiques, la lampe et le dépôt alimentaire. Il est difficile d'interpréter la situation des quelques perles situées en amont et à gauche du crâne; elles peuvent avoir été placées à côté du corps, ou avoir orné la chevelure. Quelques rares accessoires vestimentaires pourraient aussi avoir été déposés à côté du corps, en témoigne la boucle de ceinture de la sépulture SP27 du Trillet, située latéralement et à l'écart de l'extrémité proximale du fémur droit. Néanmoins, celle-ci peut avoir glissé dans la mesure où le corps s'est décomposé en espace vide.

Les coffrets ont rarement été identifiés dans les sépultures du Bas-Empire et on mentionnera ceux de Colchester en Angleterre, datés des IV $\mathrm{V}^{\mathrm{c}} \mathrm{V}^{\mathrm{c}} \mathrm{s}$. (Crummy et al., 1993, p. 148).

Les lampes ne sont présentes que dans deux sépultures d'enfants du Pillon.

Des petits clous à pointe recourbée et à tête arrondie attestent la présence de chaussures sur les sites du Pillon et du Trillet. Dans les sépultures antiques, il est fréquent que les chaussures ne soient pas portées, mais placées de part et d'autre des pieds (Crummy et al., 1993, p. 42 ; Tranoy, Gisclon, 1995 ; nécropole de J. Brel à Saintes : B. Farago com. pers.), mais des cas existent où les défunts étaient chaussés (Crummy et al., 1993, p. 42). Il n'est pas possible de le vérifier dans le cas des sépultures SP504 et SP512 du Pillon, les os des pieds n'étant pas conservés, mais dans les sépultures SP515 et SP508 et dans celles du Trillet, les clous retrouvés sont en bon rapport de situation avec les os des pieds et les chaussures pourraient donc avoir été portées.

F. B., C. B., M. R.

\section{Les dépôts alimentaires}

Les dépôts alimentaires, constitués des restes de volatiles, restent rares, avec un exemple au Pillon (SP504) et un autre au Trillet (SP16). Dans les deux cas, ils ne se trouvent pas dans un récipient en céramique. Les os du volatile de la sépulture SP504 du Pillon ont été déposés dans le coffret situé contre le contenant du corps; le mode de répartition de ces os dessine des effets de délimitation affectant une forme globalement carrée, comme si le dépôt avait été effectué dans un plat en matière périssable. Les os du volatile de la sépulture SP16 sont placés sur le fond de la fosse, en amont d'une cruche en céramique ; là encore, il n'est pas exclu qu'ils se soient trouvés à l'origine dans un plat en matière organique. Ces dépôts concernent un enfant (SP504) et une femme (SP16).

\section{SYNTHÈsE}

Les bris observés sur la vaisselle forment deux catégories, la première se rapporte à des récipients non pas fracturés mais mutilés, dont les fragments manquants sont absents dans la fosse, la seconde se compose de vases complets ou ébréchés qui sont fragmentés sur place.

La mutilation des céramiques est un phénomène fréquemment attesté dans les sépultures antiques, du Haut et du Bas-Empire. Aux Plantées à Briord dans l'Ain, des amputations de col de cruches sont notées (Perraud, 1974) ; dans le Var, la nécropole de La Calade à Cabasse a livré une sépulture en coffre du Bas-Empire renfermant des vases ébréchés dont une cruche transpercée d'un clou (Bérard, 1963); de même à Lyon, sur le site du quai Arloing on ne trouve que neuf vases complets sur quarante-deux (Tranoy, Gisclon, 1995, p. 211). 
Plusieurs exemples de cruches décapitées ou perforées sont relevés au Haut-Empire, dans le Bas-Dauphiné à Aoste (Jospin, 1998-1999, p. 22-28), dans l'Indre à Argentomagus (Allain et al., 1992, p. 126), dans la Drôme au Valladas (Bel, 1992, p. 149) et à Lyon, La Favorite (Tranoy, 1995, p. 810). De tels phénomènes sont également observés sur le mobilier en verre, par exemple une œnochoé dont il manque l'anse au Valladas (Bel, 1992, p. 149) ou quelques balsamaires dans les dépôts de crémation du Haut-Empire du site des Girardes. Des dépôts de vaisselle brisée ont été reconnus sur la nécropole du Haut-Empire des Cordiers à Mâcon en Saône-et-Loire (Barthélémy, Depierre, 1990, p. 68). Une sépulture récemment fouillée dans le Puy-de-Dôme et datée de la transition des II ${ }^{\mathrm{e}}-\mathrm{I}^{\mathrm{er}} \mathrm{s}$. avant J.-C. a livré un pot à solides et une cruche dont le bec présente une fracture en « $V$ » et dont l'anse est absente (Le Brézet à Clermont-Ferrand : fouilles inédites G. Vernet, étude F. Blaizot). Enfin, de nombreux parallèles peuvent être trouvés dans la littérature ethnologique, correspondant à autant d'explications ou presque de cette pratique (Grinsell, 1961 ; Maertens, 1979, p. 110-118) jusque chez les Pygmées qui ébrèchent des vases avant de les déposer dans les tombes (Leroy, in: Alihanga, 1979, p. 277) et chez les Inuits qui les percent de manière à éviter qu'ils soient récupérés par les vivants pour leur usage personnel (Saladin-d'Anglure, 1999).

On trouve, chez les différents auteurs qui ont observé des récipients brisés ou perforés, une nette tendance à les interpréter comme ayant trait au rituel funéraire; néanmoins, d'autres semblent privilégier l'hypothèse d'une utilisation, pour les morts, d'une céramique cassée dans le cadre domestique (Bel, 1992, p. 149; Crummy et al., 1993 ; Tranoy, Gisclon, 1995, p. 223).

Il est certain qu'une grande partie de la vaisselle déposée dans les sépultures décrites ici provient du vaisselier domestique; non seulement il n'existe aucune forme spécifique, mais de plus certains objets présentent des marques d'utilisation, telles que des réoxydations et des traces de carbone sur le fond des plats en céramique, dues à de multiples passages au feu (sépultures SP508 et SP514 du Pillon). Cependant, nous estimons être en mesure de réunir suffisamment d'éléments pour éliminer l'idée que les mutilations sont systématiquement le résultat d'un usage domestique et envisager que les céramiques ont fait l'objet d'un traitement particulier à l'occasion des funérailles. L'hypothèse de bris rituels, fréquemment émise en contexte funéraire, paraît ici probable en raison du caractère spécifique et systématique des cassures quels que soient les types et les catégories de céramiques ${ }^{5}$. En raison de leur localisation sur la partie haute des vases, ces derniers sont inutilisables dans la plupart des cas. Il convient de préciser que sept céramiques sur les dix dont une partie de la panse manque proviennent d'exemplaires retrouvés brisés dans la tombe; or, dans la mesure où ces deux phénomènes (vases mutilés non fragmentés/vases fragmentés sur place) pourraient relever de deux pratiques distinctes (cf. supra), la fréquence des lacunes de panse ne se rapporte peut-être pas à une mutilation du vase strictosensu, mais à une "perte " ou en tout cas au dépôt partiel de l'objet brisé à l'extérieur. Au Pillon, dans le cas des céramiques brisées sur place, le fait qu'elles ne se retrouvent que dans un groupe de sépultures et qu'elles soient toujours placées aux pieds du squelette constitue des caractères récurrents, qui minimisent l'hypothèse d'un bris accidentel causé par l'effondrement du couvercle des contenants.

On se gardera néanmoins de tomber dans l'excès du rituel " à tout crin " et de suivre certains auteurs pour lesquels chaque tesson issu dc la fosse est significatif d'un acte volontaire et codifié. La poignée de fragments appartenant à deux amphores, qui jonchent le fond de la fosse de la sépulture SP512 du Pillon, ne saurait être interprétée en ce sens. De manière générale, l'idée que de simples tessons trouvés dans la tombe puissent refléter un rituel spécifique (Fingerlin, 1971; Perraud, 1974; Ament, 1976, p. 289; Schweitzer, 1984, p. 94) nous semble, sinon pouvoir totalement être rejetée, en tout cas difficile à argumenter; on notera de plus qu'elle est le plus souvent énoncée a priori et ne repose pas sur une étude raisonnée de la représentation de ce mobilier. Quoi qu'il en soit on émettra de fortes réserves lorsque ce sont des tessons provenant du comblement des fosses, qui sont sans aucune autre forme de procès interprétés comme " un nouveau rite funéraire bien connu " (Châtelet, 1998, p. 27), d'autant que les auteurs s'appuient majoritairement sur des sépultures issues d'un

5. Il en est de même pour les balsamaires en verre de l'ensemble funéraire du Haut-Empire des Girardes, dont certains, par ailleurs non brisés, présentent ce type de cassure en « $V$ » sur la lèvre. On mentionnera également trois assiettes en sigillée provenant de l'ensemble funéraire des Girardes, daté du I $^{\mathrm{er}} \mathrm{s}$., qui comportent une cassure de la lèvre. 
contexte d'habitat ou proches de l'habitat (Châtelet, op. cit.; Schweitzer, 1984). Dans ces circonstances, qui en l'occurrence sont celles des ensembles funéraires examinés ici, où se trouve le matériel résiduel, lorsque les couches d'occupation ont disparu, sinon piégé dans le remplissage des structures en creux? C'est probablement là l'explication la plus raisonnable que l'on puisse fournir et c'est celle que nous retiendrons pour la sépulture SP512 du Pillon.

Ces bris et mutilations d'objets ne peuvent trouver d'explication en l'absence de témoignages directs de ceux qui les ont pratiqués. $\mathrm{Si}$, pour certains, ils symbolisent « la brièveté de la vie, la bonne chance, voire la mort elle-même - c'est-à-dire le non-sens - » (Crummy et al., 1993), ils illustrent, dans de nombreuses sociétés, la transmission des biens du défunt aux survivants et, en détournant certains de cette passation, ils permettent la circulation normale de la plupart d'entre eux (Maertens, 1979, p. 110-118). Néanmoins, nous avons distingué, dans l'étude du site du Pillon, les céramiques mutilées des céramiques brisées sur place et proposé l'hypothèse que chaque cas de figure se rapporte à deux rites différents, le premier intéressant l'offrande faite au mort et, le second, la relation du défunt avec les vivants par le biais d'un repas ou de son substitut. Il s'agit là bien entendu d'une proposition fondée sur quelques pratiques mentionnées ailleurs (Darembert, Saglio, 1969, p. ${ }^{1378-1379) . ~ L o r s q u e ~ l e ~ m o r t ~ e ́ t a i t ~ p l a c e ́ ~ d a n s ~ l a ~ t o m b e, ~}$ la coutume voulait qu'on lui offre des libations, puis les vases ayant servi à cet usage étaient fréquemment brisés et les débris rejetés avec le corps, tandis que les récipients utilisés lors du «banquet » étaient placés sur le contenant, souvent brisés intentionnellement. On ne peut que noter la coincidence qui existe entre les faits archéologiques relevés au Pillon et le rituel mentionné par ces auteurs.

Des vases de "second choix ", comme celui de la sépulture SP11 du Trillet, sont parfois mentionnés dans les tombes. Ces vases pourraient être des ratés d'ateliers dans la mesure où ils présentent des déformations, des traces de doigts et des empâtements. Par exemple, l'ensemble funéraire du Haut-Empire de Pourliat à Beaumont (Puy-de-Dôme) renferme plusieurs récipients en sigillée du sud de la Gaule, qui se caractérisent par ces aspects (Wittmann, 2000, p. 96). L'état de ces céramiques les rend probablement impropres à la consommation usuelle et l'hypothèse de vases achetés " au rabais » en vue de les déposer dans les tombes peut être envisagée, comme le propose J.-P. Jospin pour certains exemplaires d'Aoste (Jospin, 1998-1999, p. 21).

Seules les sépultures des femmes et des enfants possèdent du mobilier métallique et seuls les enfants sont accompagnés d'objets métalliques ne se rapportant pas à des accessoires vestimentaires ou à de la parure (lampes). Dans les ensembles funéraires antiques, il est fréquent que le mobilier dévolu aux femmes et aux enfants soit plus diversifié, sinon plus beau (Allain et al., 1992, p. 124-126). En revanche, aucune distinction par les céramiques n'est opérée entre les sexes et les stades de maturation. L'homogénéité du matériel céramique par ensemble est un phénomène attesté dans les séries archéologiques; ce mobilier paraissant fonctionner indépendamment des marqueurs socio-économiques (Crummy et al., 1993, p. 44), il semblerait constituer un palier inévitable et relativement codifié du strict rituel funéraire.

L'organisation de l'ensemble funéraire du Pillon est très particulière, avec une répartition spatiale en petites unités. L'analyse de la chorologie de différents paramètres permet de scinder l'ensemble funéraire en deux parties correspondant globalement à des sous-ensembles, l'une assimilable au groupe ouest et l'autre rassemblant les tombes situées à l'est et au centre. Cette répartition n'est en aucun cas fondée sur le sexe et l'âge, les femmes, les hommes et les enfants se distribuant indifféremment dans chacun de ces groupes. En revanche, une différence systématique dans le traitement des individus immatures et adultes est observée entre ces deux groupes, caractérisée par l'orientation des corps. On remarque également que les tombes situées à l'est sont "plus riches " que les autres, dans le sens où elles renferment un mobilier de parure abondant et que les modes d'inhumation sont plus diversifiés (l'ouest ne comprend que des cercueils cloués). Enfin, on a vu que la présence de céramiques brisées intentionnellement concerne les tombes placées à l'est. De même, les sépultures des Girardes s'organisent en deux groupes, l'un au nord et l'autre au sud, et seul le premier contient les sépultures pourvues de mobilier (six sur neuf) avec des types d'architecture différents. Une telle répartition est absente au Trillet. On peut noter, à titre comparatif, au moins un exemple d'une nécropole dans laquelle l'orientation des corps paraît marquer une différenciation entre les individus, dans la mesure où elle est corrélée avec un autre paramètre. Il s'agit du site des 
Quarante Mille Morts à La Boisse (Ain) daté des III'-IVes., où les squelettes dont la tête est placée à l'est sont accompagnés d'un mobilier important tandis que ceux qui ont la tête à l'ouest en sont dépourvus (Morel, Gruyer, 1961).

\section{« RECRUTEMENT » DES ENSEMBLES FUNÉRAIRES À PARTIR DES DONNÉES DÉMOGRAPHIQUES}

Conjointement à l'apport des données de l'archéologic, la compréhension d'un gisement funéraire impose parfois un recours à l'anthropologie biologique. Nous abordons donc maintenant ce domaine avec pour objectif d'étudier ce qu'il est communément convenu d'appeler le « recrutement » d'un ensemble funéraire. En d'autres termes, il s'agit d'approcher au mieux les caractéristiques biologiques propres à chaque individu et, par extension, au groupe d'individus constituant chaque population inhumée et, à partir de là, vérifier si les populations archéologiques ćtudiées sont représentatives d'une population vivante théorique ou bien, au contraire, si elles relèvent d'une éventuelle spécialisation. Plusieurs critères biologiques fondamentaux peuvent être exploités : âge et sexe, lésions spécifiques liées à la cause de la mort, état sanitaire de fond de la population ou encore certains particularismes osseux, tels les caractères discrets.

Dans le cadre de cette étude, nous avons décidé de nous consacrer à l'étude des caractéristiques démographiques. En effet, sur la base d'observations précédemment acquises pour les sites du Trillet à Meyzieu (BenNcer, 1992) et du Pillon à Marennes (Blaizot, in : Martin et al., 1994), le bilan obtenu sur les caractères discrets nous est apparu très maigre. Leur répartition sur le site du Trillet fait apparaître un regroupement de tombes sur la base de deux caractères (suture métopique et osselets de la lambdoïde) mais, dans l'état actuel de nos connaissances, leur signification demeure incertaine (il pourrait très bien résulter d'un regroupement aléatoire) et nous avons préféré abandonner cette recherche. Par ailleurs, l'étude de la pathologie dentaire et osseuse menée uniquement sur le site du Trillet a livré des observations relativement classiques avec son quota habituel de lésions dentaires, dégénératives, traumatiques et autres (BenNcer, 1992). Cependant le dépistage récent d'éventuelles lésions pathologiques rares (il pourrait s'agir de traces syphilitiques), présentes sur trois individus, demande a être vérifié, ce qui nous oblige à repousser pour l'instant cette recherche qui, si elle s'avère justifiée, fera l'objet d'une publication ultérieure.

\section{LES PARAMÈTRES DÉMOGRAPHIQUES}

Les trente-deux individus du Trillet sont relativement bien conservés et, fait remarquable, le gisement a pu être exploré dans sa totalité. Malheureusement, l'état de conservation et de représentation des ossements du Pillon et des Girardes est très mauvais, tout particulièrement aux Girardes. Associé à un très faible effectif, respectivement quinze et quatorze sépultures, cet état ne nous permet pas d'étudier de manière satisfaisante les individus issus de ces deux sites.

Le tout premier travail consiste à fournir les données brutes concernant le stade de maturité, l'estimation du sexe des adultes et l'estimation de l'âge au décès des individus adultes et immatures. Selon la catégoric étudiée (enfants, adolescents, adultes) les méthodes sont nombreuses mais de valeurs diverses, et une certaine prudence doit être de rigueur. Les méthodes sont choisies en fonction de la conservation du matéricl et identiques sur les trois gisements étudiés.

\section{ESTIMATION DU STADE DE MATURITÉ}

Nous avons tout d'abord tenté de séparer les squelettes adultes des immatures. Lorsque la conservation des os est suffisante, la reconnaissance du caractère adulte d'un squelette ne pose généralement pas de problème, mais il faut admettre que, dans certains cas, l'absence d'ćléments déterminants ne nous permet pas toujours de. décider, tout particulièrement lors de la période charnière grands adolescents-adultes.

$\mathrm{Au}$ Trillet, les individus se répartissent en dix-sept adultes, douze immatures et trois indéterminés. Parmi ces derniers, l'un a été estimé comme adulte possible (SP4) (BenNcer, 1992), les deux autres sépultures (SP14 et SP25) ont été détruites par les labours (Frascone, Staniaszek, 1992) et les quelques fragments d'os restants ne nous permettent pas de trancher entre le stade adulte ou adolescent. 
Parmi les quinze individus du Pillon nous avons déterminé la présence de sept adultes et cinq immatures ; là encore subsistent trois indéterminés, l'un est représenté seulement par quelques fragments d'os (SP503), nous ne pouvons donc trancher sur le caractère tout à fait mature de cet individu, pour les deux autres (SP506 et SP511) l'état des esquilles osseuses interdit toute tentative d'estimation. Seul le petit format de la fosse de SP506 permettrait d'attribuer un caractère immature à cet individu.

Le site des Girardes a livré un échantillon de quatorze sépultures à inhumation (deux autres concernent des inhumations de chiens), dont cinq ne contenaient aucun ossement. Elles ont été identifiées en tant que sépultures en raison de la taille et de la situation des fosses dans la nécropole. Deux d'entre elles, SP5021 et SP5067, de petites dimensions, peuvent être attribuées à des sujets immatures. Parmi les neuf sépultures restantes, une seule (SP5002) possédait un squelette suffisamment bien représenté pour être attribuée à un individu mature.

\section{ESTIMATION DU SEXE DES ADULTES}

La plus fiable des méthodes ostéoscopiques fait intervenir, bien entendu, les observations portant sur les bassins adultes. La méthode visuelle de J. Bruzek (1991), fondée sur de nombreuses observations morphologiques portant sur l'os coxal, nous a permis d'estimer le sexe d'un certain nombre d'individus avec une relative sécurité. Lorsque les os coxaux étaient fragmentaires, nous avons utilisé une nouvelle approche de diagnose sexuelle portant sur trois caractères morphologiques de la face sacro-pelvienne de l'os coxal (Bruzek et al., 1996). Ainsi au Trillet, les estimations sexuelles les plus fiables concernent onze sujets adultes sur dix-sept, soit huit hommes et trois femmes. Pour Le Pillon un seul individu sur sept a été attribué au sexe masculin. De même aux Girardes où un seul individu sur quatorze s'est révélé féminin.

Beaucoup de bassins étant absents ou trop abîmés les résultats sont bien limités, tout particulièrement pour Le Pillon et Les Girardes. Donc, indépendamment des os coxaux et lorsque la conservation des restes humains le permettait, nous avons relevé les caractères sexuels secondaires du crâne, de la mandibule et du fémur (Ferembach et al., 1979) afin d'établir des rapports de gracilité et de robustesse. Ces caractères « extra-coxaux » sont généralement considérés comme moins performants car essentiellement liés à la robustesse et au format, nous devons donc les considérer à un degré de fiabilité nettement moindre. Les caractères retenus n'ont pas tous la même importance, ceux affectés d'un "poids" maximum sont plus fréquents sur le crâne. Nous considérerons comme masculin ou féminin possible ( $\mathrm{M}$ ?, $\mathrm{F}$ ?) les individus dont le crâne est bien représenté. Les observations morphologiques réalisées sur la mandibule et/ou le fémur ne seront prises en compte qu'à un troisième niveau de fiabilité, bien entendu le plus incertain ( $M$ ?, $F$ ?).

$\mathrm{Au}$ Trillet, les résultats obtenus confirment l'écart observé à partir de l'étude des os coxaux, l'effectif potentiel des hommes est porté à onze individus, celui des femmes à cinq ; il subsiste un indéterminé. Au Pillon, parmi les six adultes de sexe indéterminé, trois individus pourraient appartenir au sexe féminin, deux autres au sexe masculin. Sur la base des deux méthodes d'estimation, nous aurions donc trois femmes pour trois hommes; là encore subsiste un indéterminé. Aux Girardes, aucune observation ostéologique supplémentaire ne nous permet de pousser plus loin notre tentative d'estimation. Seul un individu (SP5003) serait susceptible d'appartenir à la classe féminine en raison d'un matériel funéraire typique ( $c f$. supra). Les résultats sont consignés dans les tableaux III, IV et V.

\section{ESTIMATION DE L'ÂGE AU DÉCÈs DES ADULTES}

Ces dernières années, plusieurs études portant sur l'estimation de l'âge au décès des adultes ont révélé que les indicateurs d'âge fondés sur des critères de vieillissement progressif du squelette étaient très difficiles à apprécier ( $c f$. revue exhaustive d'Iscan ed., 1989; Saunders et al., 1992). Les méthodes classiques faisant intervenir le degré de synostose des sutures crâniennes tant endocrâniennes qu'exocrâniennes, les modifications du tissu spongieux des extrémités proximales fémorales et humérales, les changements morphologiques affectant la surface auriculaire de l'ilium et la surface symphysaire du pubis ont révélé de bien piètres résultats avec une marge d'erreur très importante dès lors qu'on les appliquait à des populations d'âge et de sexe connus (Rougé, 1993 ; Key et al., 1994; Broqua, 1998). Un autre problème concerne l'état de conservation du matériel anthropologique; généralement très 
Tabl. III - Le Trillet à Meyzieu. Estimation du sexe et de l'âge au décès (réalisation : D. Castex).

\begin{tabular}{|c|c|c|c|c|c|}
\hline Sép. & Maturité & Critères d'estimation de l'âge & Classe d'âge & Critères d'estimation du sexe & Sexe \\
\hline 1 & Adulte & - & 1 & - & 1 \\
\hline \multirow[t]{2}{*}{2} & Immature & Âge dentaire (maxillaire et mandibule) & $10-14$ ans & - & - \\
\hline & & Âge diaphysaire (radius gauche) & & & \\
\hline \multirow[t]{2}{*}{3} & Immature & Âge dentaire (maxillaire et mandibule) & $10-14$ ans & - & - \\
\hline & & Âge diaphysaire (tous les os longs côté droit) & & & \\
\hline 4 & Adulte? & - & - & - & - \\
\hline \multirow{2}{*}{5} & Adulte & Point sternal de la clavicule soudé & + de 30 ans & Caractères extra-coxaux & $\mathrm{F}$ ? \\
\hline & & & & (frontal et tête fémorale) & \\
\hline \multirow[t]{2}{*}{6} & Immature & Âge dentaire (maxillaire et mandibule) & $10-14$ ans & - & - \\
\hline & & Âge diaphysaire (humérus, fémur et tibia droits) & & & \\
\hline \multirow[t]{2}{*}{7} & Immature & Âge dentaire (maxillaire et mandibule) & $10-14 / 15-19$ ans & - & - \\
\hline & & Âge diaphysaire (radius et fémur gauches ; tibia droit) & & & \\
\hline 8 & Adulte & Point sternal de la clavicule soudé & + de 30 ans & Caractères coxaux & M \\
\hline 9 & Adulte & - & 1 & Caractères coxaux & M \\
\hline 10 & Adulte & - & 1 & Caractères coxaux & M \\
\hline \multirow[t]{2}{*}{11} & Adulte & $\overline{-}$ & 1 & Caractères extra-coxaux & $M ?$ \\
\hline & & & & (crâne, mandibule et fémur) & \\
\hline 12 & Adulte & Point sternal de la clavicule soudé & + de 30 ans & Caractères coxaux & M \\
\hline \multirow[t]{3}{*}{13} & Adulte & Plusieurs signes de sénescence & + de 30 ans & Caractères coxaux & $M$ \\
\hline & & État dentaire très médiocre (nombreuses pertes ante-mortem) & & & \\
\hline & & Lésions dégénératives prononcées sur l'ensemble du rachis & & & \\
\hline 14 & Adulte? & - & - & - & - \\
\hline \multirow[t]{2}{*}{15} & Immature & Âge dentaire (maxillaire et mandibule) & $0-1 / 1-4$ ans & - & - \\
\hline & & Âge diaphysaire (fémur droit) & & & \\
\hline 16 & Adulte & - & 1 & Caractères coxaux & $\mathrm{F}$ \\
\hline \multirow[t]{3}{*}{17} & Adulte & Quelques infections dentaires & + de 30 ans & Caractères coxaux & M \\
\hline & & État du spongieux des têtes humérales et fémorales & & & \\
\hline & & État de la symphyse pubienne & & & \\
\hline \multirow[t]{2}{*}{18} & Adulte & 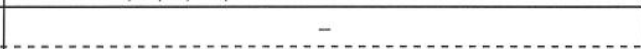 & + de 30 ans & Caractères coxaux et extra-coxaux & $\mathrm{F} ?$ \\
\hline & & & & (arc composé de l'ilion, crâne et mandibule) & \\
\hline \multirow[t]{2}{*}{19} & Adulte & État dentaire médiocre (caries et pertes ante-mortem) & + de 30 ans & Caractères extra-coxaux & $M ?$ \\
\hline & & Arthroses et enthésopathies en plusieurs points du squelette & & (crâne, mandibule et fémur) & \\
\hline \multirow[t]{2}{*}{20} & Adulte & Arthroses prononcées sur vertèbres lombaires & + de 30 ans & Caractères extra-coxaux & $M ?$ \\
\hline & & État de la symphyse pubienne et de la surface auriculaire & & (mandibule) & \\
\hline 21 & Adulte & - & + de 30 ans & Caractères coxaux & $\mathrm{M}$ \\
\hline \multirow[t]{2}{*}{22} & Adulte & Point sternal de la clavicule et crête iliaque non soudés & - de 30 ans & Caractères coxaux & $F$ \\
\hline & & Lignes épiphysaires des têtes fémorales encore perceptibles & & & \\
\hline 23 & Immature & Âge dentaire (maxillaire et mandibule) & $0-1 / 1-4$ ans & - & - \\
\hline 24 & Adulte & - & 1 & Caractères coxaux et extra-coxaux & $M$ \\
\hline 25 & Adulte? & - & - & - & - \\
\hline 26 & Immalure & Âge denlaire (maxillaire el mandibule) & $5-9 / 10-14$ ans & - & - \\
\hline \multirow[t]{2}{*}{27} & Immature & Âge dentaire (maxillaire et mandibule) & $10-14 / 15-19$ ans & - & - \\
\hline & & Âge diaphysaire (radius et tibia droits) & & & \\
\hline 28 & Immature & Âge dentaire (maxillaire et mandibule) & $0-1 / 1-4$ ans & - & - \\
\hline \multirow[t]{3}{*}{29} & Adulte & État dentaire médiocre (quelques pertes ante-mortem) & + de 30 ans & Caractères coxaux & $\mathrm{F}$ \\
\hline & & Lésions dégénératives sur l'ensemble du rachis & & & \\
\hline & & État de la symphyse pubienne et de la surface auriculaire & & & \\
\hline \multirow[t]{2}{*}{30} & Immature & Âge dentaire (maxillaire et mandibule) & $0-1 / 1-4$ ans & - & $\overline{-}$ \\
\hline & & Âge diaphysaire (fémur droit) & & & \\
\hline \multirow[t]{2}{*}{31} & Immature & Âge dentaire (maxillaire et mandibule) & $10-14$ ans & - & $=$ \\
\hline & & Âge diaphysaire (fémur gauche et tibia droit) & & & \\
\hline 32 & Immature & Âge dentaire (maxillaire et mandibule) & $0-1 / 1-4$ ans & - & - \\
\hline
\end{tabular}


Tabl. IV - Le Pillon à Marennes. Estimation du sexe et de l'âge au décès : Ad., adulte; Im., immature (réalisation: D. Castex).

\begin{tabular}{|c|c|c|c|c|c|}
\hline Sép. & Maturité & Critères d'estimation de l'âge & Classe d'âge & Critères d'estimation du sexe & Sexe \\
\hline \multirow[t]{3}{*}{502} & Ad. & Point sternal clavicule soudé & + de 30 ans & Caractères coxaux et extra-coxaux & $M$ ? \\
\hline & & & & (arc composé et sillon préauriculaire) & \\
\hline & & & & (bloc cranio-facial et mandibule) & \\
\hline \multirow[t]{2}{*}{503} & Ad. ? & Un fragment de diaphyse d'humérus & 1 & (1) & $-\ldots$ \\
\hline & & Ossements très altérés (métatarsiens, partie distale d'un radius) & & & \\
\hline 504 & Im. & Âge dentaire (maxillaire et mandibule) & $10-14$ ans & - & - \\
\hline 505 & Im. & Âge diaphysaire (tibia droit) & 5-9/10-14 ans & - & - \\
\hline 506 & Im. ? & Fosse de petit format & 1 & - & - \\
\hline 507 & Im. & Âge dentaire (maxillaire et mandibule) & $5-9 / 10-14$ ans & - & - \\
\hline \multirow[t]{2}{*}{508} & Ad. & Points sternaux clavicules non soudés & - de 30 ans & Caractères extra-coxaux & $F ?$ \\
\hline & & & & (bloc cranio-facial et mandibule) & \\
\hline \multirow[t]{2}{*}{509} & Ad. & Point sternal clavicule soudé & + de 30 ans & Caractères coxaux et extra-coxaux & $M$ \\
\hline & & & & (bloc cranio-facial et mandibule) & \\
\hline 510 & Im. & Âge dentaire (maxillaire et mandibule) & $5-9$ ans & - & - \\
\hline 511 & $?$ & - & 1 & - & - \\
\hline 512 & Ad. & - & 1 & - & 1 \\
\hline \multirow[t]{2}{*}{513} & Ad. & Point sternal clavicule soudé & \pm de 30 ans & Caractères coxaux et extra-coxaux & $F ?$ \\
\hline & & & & (bloc cranio-facial et mandibule) & \\
\hline \multirow[t]{2}{*}{514} & Ad. & Point sternal clavicule soudé & + de 30 ans & Caractères coxaux et extra-coxaux & $M ?$ \\
\hline & & & & (bloc cranio-facial et mandibule) & \\
\hline \multirow[t]{2}{*}{515} & Ad. & Point sternal clavicule soudé & + de 30 ans & Caractères coxaux et extra-coxaux & $F ?$ \\
\hline & & & & (bloc cranio-facial et mandibule) & \\
\hline 516 & Im. & - & 1 & - & - \\
\hline
\end{tabular}

Tabl. V - Les Girardes à Lapalud. Estimation du sexe et de l'âge au décès (réalisation: D. Castex).

\begin{tabular}{|c|c|c|}
\hline Sép. & 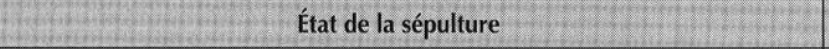 & Estimations maturité/âge/sexe \\
\hline 5001 & Squelette exhumé lors de la prospection. Aucun ossement n'a pu être étudié & Adulte, âge et sexe indéterminés \\
\hline 5002 & Squelette bien représenté & Adulte âge indét. - Sexe F (caractères coxaux) \\
\hline 5007 & Absence d'os, identification sépulcrale : taille et situation & Maturité, âge et sexe indéterminés \\
\hline 5008 & Absence d'os, identification sépulcrale : taille et situation & Maturité, âge et sexe indéterminés \\
\hline 5021 & Absence d'os, taille de la fosse suggère un enfant & Maturité, âge et sexe indéterminés \\
\hline 5022 & Seulement 1 ou 2 diaphyses & Maturité, âge et sexe indéterminés \\
\hline 5023 & Seulement 1 ou 2 diaphyses & Maturité et âge indét. - Sexe F ? (sexe archéologique) \\
\hline 5038 & Diaphyses d'os longs et éléments de la voûte crânienne & Maturité, âge et sexe indéterminés \\
\hline 5039 & Absence d'os, identification sépulcrale : taille et situation & Maturité, âge et sexe indéterminés \\
\hline 5041 & Diaphyses d'os longs et éléments de la voûte crânienne & Maturité, âge et sexe indéterminés \\
\hline 5042 & Diaphyses d'os longs et éléments de la voûte crânienne & Maturité, âge et sexe indéterminés \\
\hline 5043 & Seulement l'écaille occipitale & Maturité, âge et sexe indéterminés \\
\hline 5058 & Seulement l'écaille occipitale & Maturité, âge et sexe indéterminés \\
\hline 5062 & Absence d'os, identification sépulcrale : taille et situation & Maturité, âge et sexe indéterminés \\
\hline 5067 & Absence d'os, taille de la fosse suggère un enfant & Maturité, âge et sexe indéterminés \\
\hline
\end{tabular}


variable, il est souvent impossible d'observer ces indicateurs d'âge sur tous les sujets. Pour toutes ces raisons, nous avons choisi de distribuer tous les individus adultes de nos séries selon deux grandes classes d'âges : d'une part, les individus de moins de 30 ans, classe généralement bien caractériséc puisqu'elle correspond à la phase de disparition des dernières traces d'immaturité comme le point sternal de la clavicule et la crête iliaque (Owings-Webb, Suchey, 1985) facilement repérables sur le squelette et, d'autre part, les individus de plus de 30 ans.

Sur ces bases le site du Trillet nous a livré un seul individu de moins de 30 ans (SP22) et trois adultes de plus de 30 ans (SP5, SP8 et SP12). Pour les treize sujets restants d'âge indéterminé, nous avons recherché les individus pour lesquels nous pouvions supposer un âge supérieur à 30 ans. Nous nous sommes fondés sur un faisceau d'arguments concordants concernant l'état général de l'apparcil dentaire (caries, abcès, perte ante mortem), la présence de lésions dégénératives de type arthrose et enthésopathic, ainsi que des observations de plusieurs sites osseux évoluant avec l'âge (symphyse pubienne et surface auriculaire des os coxaux). Nous sommes conscients qu'il s'agit là davantage d'une intuition que d'une véritable méthode, mais pour certains des sujets d'âge indéterminé, les observations, tant dentaires qu'osseuses, laissent peu de doutes quant à leur statut d'adultes clairement matures, voire âgés. L'effectif des plus de 30 ans s'est trouvé ainsi augmenté de sept sujets; seuls six individus n'ont pas pu être attribués à une des deux classes d'âge adulte.

Pour Le Pillon, la conservation exceptionnelle d'un grand nombre de clavicules nous a permis de pouvoir déterminer les individus de moins de 30 ans. Sur les six adultes possédant leurs clavicules, un seul se situe en deçà des 30 ans (SP508). Aux Girardes, aucun âge n’a pu être estimé. Les résultats obtenus sont consignés dans les tableaux III, IV et V.

\section{ESTIMATION DE L'ÂGE AU DÉCÈS DES IMMATURES}

L'estimation de l'âge au décès des sujets immatures est généralement plus précise que celle des adultes car elle repose sur l'observation de processus de croissance assez peu variables d'un individu à l'autre. Nous avons privilégié dans un premicr temps les méthodes fondées sur le stade de maturation dentaire. Nous avons conservé les données brutes obtenues par A. BenNcer (1992) par la méthode de Ubelaker (1978), mais nous les avons complétées systématiquement pour les individus de moins de cinq ans et, lorsque cela était possible (présence des canines et des molaires mandibulaires déciduales), par des observations à partir de la méthode de Moorrees, Fanning et Hunt (1963). À ces estimations ont pu être ajoutées des mesures effectuées sur plusieurs éléments du squelette en se référant aux tableaux de Sundick (1978) et à des bases de données établies pour des populations médiévales (Castex, 1994).

Nous avons ensuite ventilé tous les individus immatures selon les classes d'âge $0,1-4,5-9,10-14$ et 15-19 ans (âge en années révolues), conformément aux tables de mortalité de Ledermann (1969), ceci afin d'analyser la structure démographique globale des décès des sujets immatures. Les estimations de l'âge étant toujours données sous forme d'intervalle (que ce soit d'après le stade de maturation dentaire ou à partir des longueurs diaphysaires des os longs), les individus ont été distribués dans une classe bien précise ou dans deux classes successives.

$\mathrm{Au}$ Trillet, tous les sujets immatures (soit douze individus) ont pu bénéficier d'une estimation d'âge à partir du stade d'éruption et de calcification dentaire, huit seulement à partir de la mensuration des os longs. Ainsi quatre ont pu être répartis sans ambiguité dans une classe d'âge, les huit autres se situant dans deux intervalles successifs. Pour Le Pillon, où l'effectif des sujets immatures est très faible, nous avons pu estimer l'âge de quatre individus sur cinq, trois en combinant la maturation dentaire et osseuse, un à partir de la maturation osseuse ; tous se concentrent dans les classes 5-9 et 10-14 ans. Compte tenu des difficultés déjà exposées pour Les Girardes, aucune étude n'a pu être effectuée. Les résultats obtenus sont consignés dans les tableaux III, IV et $\mathrm{V}$.

\section{DE L'INDIVIDU À LA POPULATION}

Les études paléodémographiques, fondées sur l'analyse de la composition par âge et par sexe des populations exhumées, font fréquemment l'objet d'une attention particulière mais il est de plus en plus clairement admis que l'on ne peut désormais ignorer certains problèmes inévitables. Le tout premier concerne l'exis- 
tence inéluctable et, dans la plupart des cas, incontrôlable de nombreux filtres (naturels, culturels...) entre la population archéologique que l'on cherche à étudier et la population d'origine dont elle est issue (Masset, 1987 ; Sellier, 1987, 1989, 1996; Masset, Sellier, 1990). Par ailleurs, même si les efforts s'intensifient et si l'on note des avancées certaines dans les techniques d'estimation de l'âge et du sexe, les indicateurs d'ordre biologique fournis par le squelette humain restent encore très imparfaits. À l'instar d'autres chercheurs et au regard des analyses paléodémographiques menées ces dernières années (Sellier, 1996; Castex, Friess, à paraître), nous sommes plutôt partisans d'en recentrer les objectifs en essayant de détecter et d'interpréter d'éventuelles anomalies démographiques et de les conjuguer aux données archéologiques.

Nous avons donc cherché à vérifier si les distributions en fonction de l'âge et du sexe obtenues pour nos échantillons archéologiques sont proches de celles que l'on peut attendre dans le cas d'une démographie naturelle ou si au contraire elles révèlent une spécialisation. À cet égard, nous nous référerons aux tables de mortalité théoriques de Ledermann (1969) actuellement les plus utilisées, surtout par les chercheurs francophones. Nous avons choisi comme indications d'entrée à ces tables l'espérance de vie à la naissance $\left(\mathrm{e}^{\circ}[0]\right)$ pour des valeurs comprises entre 20 et 40 ans, limites minimum et maximum communément admises pour les populations préjennériennes (Masset, 1975 ; Sellier, 1996).

\section{REPRÉSENTATION DES SUJETS IMMATURES PAR RAPPORT À LA POPULATION TOTALE}

La séparation des squelettes immatures et adultes nous a permis dans un premier temps de définir la représentation des individus non-adultes (des nouveau-nés jusqu'au grands adolescents) par rapport à la population totale inhumée. Dans le cadre d'un schéma de mortalité archaique, ce rapport varie entre 74 et $36 \%$ pour des espérances de vie à la naissance comprises respectivement entre 20 et 40 ans. Cette proportion se trouve en partie vérifiée au Trillet où le rapport atteint la valeur de $41,4 \%$ (douze sujets immatures pour vingt-neuf inhumés). Au Pillon, où l'effectif est très faible (cinq immatures pour douze inhumés), nous obtenons une valeur quasiment identique, soit $41,6 \%$.

\section{DISTRIBUTION SELON LE SEXE DES ADULTES}

Le nombre respectif d'hommes et de femmes obtenu au sein de chacun de nos échantillons nous permet de définir un taux de masculinité (rapport de l'effectif des hommes sur l'effectif des hommes et des femmes) et de le comparer à celui d'une démographie naturelle (taux de masculinité théorique de $50 \%$ ).

Au Trillet, la distribution sexuelle des adultes est très nettement déséquilibrée. Une première estimation fondée sur l'observation des caractères sexuels présents sur les os coxaux de onze individus sur dix-sept montre un taux de masculinité de 72,7\%. En tenant compte des caractères sexuels secondaires des os "extra-coxaux ", l'effectif potentiel des sujets masculins reste très nettement supérieur à celui des sujets féminins, le taux de masculinité reste proche de ce qu'il était précédemment, soit $68,5 \%$. Quel que soit le degré de fiabilité choisi ( $f$. supra), les valeurs obtenues sont très éloignées du taux de masculinité théorique de $50 \%$. La prudence nous incite à ne considérer que le premier niveau de fiabilité (à partir des os coxaux) et, dans ce cas, la faiblesse de l'échantillon ne permet pas de prouver une différence statistiquement significative. Malgré tout, il faudrait pour rétablir l'équilibre que l'ensemble des indéterminés soit des femmes, ce qui parait impensable en termes de probabilité, sauf si l'on admet des traitements funéraires particuliers induisant une conservation différentielle entre les sexes, ce qui ne semble pas prouvé par ailleurs (cf. supra). Ainsi donc, nous pouvons supposer que le contraste très net observé entre l'effectif des hommes et celui des femmes témoigne d'une sélection.

$\mathrm{Au}$ Pillon, les piètres résultats obtenus ne permettent pas d'aller au-delà du simple constat d'une répartition des sexes apparemment équitable. Aux Girardes, l'état des ossements nous prive de tout commentaire quant au nombre respectif d'hommes et de femmes inhumés.

\section{DISTRIBUTION DE LA POPULATION ADULTE SELON L'ÂGE AU DÉCÈS}

De la même manière que pour le rapport des sujets immatures sur l'ensemble de la population nous avons cherché à établir la représentativité des adultes de moins de 30 ans sur l'ensemble de la population adulte, l'imperfection des méthodes d'estimation de l'âge ne nous permettant pas de dépasser ce seuil de discussion. 
Toujours dans le cadre d'un schéma de mortalité archaïque, ce rapport évolue de 18 à $10 \%$ pour des espérances de vie à la naissance comprises respectivement entre 20 et 40 ans (Ledermann, 1969).

Sur la base de nos estimations ( $c f$. supra), le rapport obtenu pour Le Trillet est d'environ $9 \%$. Cette valeur apparaît faible comparée à ce qu'elle devrait être dans le cadre d'une mortalité archaiqque, même pour une espérance de vie à la naissance très élevée. Ceci révèle une mortalité des adultes non naturelle en raison d'une trop grande fréquence d'adultes âgés.

Au Pillon, malgré l'effectif restreint des individus dont l'âge a été estimé, nous pouvons envisager un rapport proche de celui d'une mortalité naturelle avec une valeur de $16,6 \%$.

Étant donné la faiblesse de nos échantillons, l'analyse de la répartition de l'âge au décès de la population adulte en fonction du sexe nous permet seulement de constater que les seuls individus adultes dont l'âge a pu être estimé à moins de 30 ans sont une femme au Trillet et un sujet supposé féminin au Pillon.

\section{DisTRIBUTION DE LA POPULATION IMMATURE SELON L'ÂGE AU DÉCÈS}

Compte tenu de la répartition des sujets immatures en classes d'âge démographiques mais également dans des interclasses, nous avons été conduits à examiner non pas une seule répartition (ce qui est classiquement effectué lorsque l'on travaille à partir de l'âge moyen d'un individu) mais plusieurs répartitions susceptibles de se produire. Pour la discussion nous avons opté pour le principe de conformité ou principe de minimalisation des anomalies (Sellier, 1996). Puisqu'a priori nous ne pouvons pas choisir une répartition plutôt qu'une autre, il paraît plus prudent de rechercher celle qui se rapproche le plus d'une distribution naturelle. Les individus se situant dans les interclasses entraînent des variations d'effectifs dans un sens ou dans l'autre (plus jeune ou plus âgé). Ces variations peuvent modifier les rapports entre les classes d'âge et donc les interprétations qui en découlent. Les différences observées peuvent être d'autant plus importantes que l'effectif des non-adultes est faible, ce qui est effectivement le cas pour nos échantillons archéologiques. En appliquant le principe de conformité, nous nous préservons d'éventuelles interprétations erronées sur la base d'anomalies supposées et nous pouvons nous consacrer aux véritables anomalies, si tant est qu'il puisse y en avoir, le seul danger étant que certaines anomalies peuvent ainsi passer inaperçues.

Nous nous sommes ensuite référés à plusieurs critères choisis dans les tables de Ledermann (1969). Premièrement, la valeur et la relation des divers quotients de mortalité (ou probabilité de mourir) pour chaque tranche d'âge (ces quotients sont généralement désignés sous la forme $\mathrm{aQx}$, où $\mathrm{x}$ désigne l'âge d'entrée dans la classe d'âge et a la durée en années de cette classe). Deuxièmement, les valeurs les plus acceptables du point de vue démographique pour le rapport de l'effectif des enfants décédés entre 5 et 9 ans sur celui des enfants décédés entre 10 et 14 ans, et le rapport de l'effectif des enfants décédés entre 5 et 14 ans sur celui des adultes décédés après l'âge de 20 ans (encore appelé indice de juvénilité). Ces critères ont été choisis pour des espérances de vie à la naissance toujours comprises entre 20 et 40 ans.

$\mathrm{Au}$ Trillet, sur douze sujets immatures, quatre ont pu être répartis sans ambiguité dans une classe d'âge, les huit autres pouvaient appartenir à deux intervalles successifs. La répartition de ces huit individus se situant entre deux classes nous amène à considérer non plus une seule distribution mais trente-six distributions différentes sur deux cent cinquante-six combinaisons possibles, certaines distributions étant redondantes (tabl. VI). Nous avons ensuite cherché à cerner la distribution la plus «normale " possible en fonction des critères choisis et de leurs valeurs respectives comparées à celles d'une mortalité naturelle donnée par les valeurs théoriques de Ledermann (1969) pour des espérances de vie à la naissance entre 20 et 40 ans. À cet effet, nous avons utilisé un traitement informatique des données permettant d'obtenir rapidement une distribution des sujets immatures selon plusieurs combinaisons (Sellier et al., 1995). La vraisemblance de ces combinaisons est alors discutée en fonction des critères précités.

Le tout premier problème concerne les cinq sujets immatures de moins de cinq ans. Leur état de conservation très moyen et les critères d'estimation de l'âge insuffisamment précis ne nous permettent pas de les attribuer avec certitude à une classe bien définie (0 ou 1-4 ans ?). Nous voyons que le choix de les distribuer dans l'une ou l'autre de ces classes peut avoir des implications importantes sur le « recrutement » (biais des 0 ?, biais des 1-4 ?). 
Tabl. VI - Les différentes possibilités de classement des sujets immatures du Trillet à Meyzieu. Conséquences sur les valeurs des quotients de mortalité et les rapports des effectifs des décès. Comparaison avec les tables types de Ledermann, 1969 (réalisation: D. Castex).

\begin{tabular}{|c|c|c|c|c|c|c|c|c|c|c|c|c|c|c|c|c|}
\hline \multicolumn{6}{|c|}{ Effectifs des classes d'âge } & \multirow[t]{2}{*}{ Comb. } & \multirow[t]{2}{*}{ Proba. } & \multicolumn{7}{|c|}{ Quotients de mortalité en \%o } & \multicolumn{2}{|c|}{ Rapports des décès } \\
\hline $0-1$ & $1-4$ & $5-9$ & $10-14$ & 15-19 & 20 et + & & & $1 \mathrm{q0}$ & $4 q 1$ & $5 q 5$ & $5 q 10$ & $5 q 15$ & $15 q 0$ & $20 \mathrm{q} 0$ & D5-9/D10-14 & D5-14/D20 et + \\
\hline 5 & 0 & 1 & 6 & 0 & 17 & 1 & 0,00391 & 172,4 & 0,0 & 41,7 & 260,9 & 0,0 & 413,8 & 413,8 & 0,17 & 0,41 \\
\hline 4 & 1 & 1 & 6 & 0 & 17 & 5 & 0,01953 & 137,9 & 40,0 & 41,7 & 260,9 & 0,0 & 413,8 & 413,8 & 0,17 & 0,41 \\
\hline 3 & 2 & 1 & 6 & 0 & 17 & 10 & 0,03906 & 103,4 & 76,9 & 41,7 & 260,9 & 0,0 & 413,8 & 413,8 & 0,17 & 0,41 \\
\hline 2 & 3 & 1 & 6 & 0 & 17 & 10 & 0,03906 & 69,0 & 111,1 & 41,7 & 260,9 & 0,0 & 413,8 & 413,8 & 0,17 & 0,41 \\
\hline 1 & 4 & 1 & 6 & 0 & 17 & 5 & 0,01953 & 34,5 & 142,9 & 41,7 & 260,9 & 0,0 & 413,8 & 413,8 & 0,17 & 0,41 \\
\hline 0 & 5 & 1 & 6 & 0 & 17 & 1 & 0,00391 & 0,0 & 172,4 & 41,7 & 260,9 & 0,0 & 413,8 & 413,8 & 0,17 & 0,41 \\
\hline 5 & 0 & 0 & 7 & 0 & 17 & 1 & 0,00391 & 172,4 & 0,0 & 0,0 & 291,7 & 0,0 & 413,8 & 413,8 & 0,00 & 0,41 \\
\hline 4 & 1 & 0 & 7 & 0 & 17 & 5 & 0,01953 & 137,9 & 40,0 & 0,0 & 291,7 & 0,0 & 413,8 & 413,8 & 0,00 & 0,41 \\
\hline 3 & 2 & 0 & 7 & 0 & 17 & 10 & 0,03906 & 103,4 & 76,9 & 0,0 & 291,7 & 0,0 & 413,8 & 413,8 & 0,00 & 0,41 \\
\hline 2 & 3 & 0 & 7 & 0 & 17 & 10 & 0,03906 & 69,0 & 111,1 & 0,0 & 291,7 & 0,0 & 413,8 & 413,8 & 0,00 & 0,41 \\
\hline 1 & 4 & 0 & 7 & 0 & 17 & 5 & 0,01953 & 34,5 & 142,9 & 0,0 & 291,7 & 0,0 & 413,8 & 413,8 & 0,00 & 0,41 \\
\hline 0 & 5 & 0 & 7 & 0 & 17 & 1 & 0,00391 & 0,0 & 172,4 & 0,0 & 291,7 & 0,0 & 413,8 & 413,8 & 0,00 & 0,41 \\
\hline 5 & 0 & 1 & 5 & 1 & 17 & 2 & 0,00781 & 172,4 & 0,0 & 41,7 & 217,4 & 55,6 & 379,3 & 413,8 & 0,20 & 0,35 \\
\hline 4 & 1 & 1 & 5 & 1 & 17 & 10 & 0,03906 & 137,9 & 40,0 & 41,7 & 217,4 & 55,6 & 379,3 & 413,8 & 0,20 & 0,35 \\
\hline 3 & 2 & 1 & 5 & 1 & 17 & 20 & 0,07813 & 103,4 & 76,9 & 41,7 & 217,4 & 55,6 & 379,3 & 413,8 & 0,20 & 0,35 \\
\hline 2 & 3 & 1 & 5 & 1 & 17 & 20 & 0,07813 & 69,0 & 111,1 & 41,7 & 217,4 & 55,6 & 379,3 & 413,8 & 0,20 & 0,35 \\
\hline 1 & 4 & 1 & 5 & 1 & 17 & 10 & 0,03906 & 34,5 & 142,9 & 41,7 & 217,4 & 55,6 & 379,3 & 413,8 & 0,20 & 0,35 \\
\hline 0 & 5 & 1 & 5 & 1 & 17 & 2 & 0,00781 & 0,0 & 172,4 & 41,7 & 217,4 & 55,6 & 379,3 & 413,8 & 0,20 & 0,35 \\
\hline 5 & 0 & 0 & 6 & 1 & 17 & 2 & 0,00781 & 172,4 & 0,0 & 0,0 & 250,0 & 55,6 & 379,3 & 413,8 & 0,00 & 0,35 \\
\hline 4 & 1 & 0 & 6 & 1 & 17 & 10 & 0,03906 & 137,9 & 40,0 & 0,0 & 250,0 & 55,6 & 379,3 & 413,8 & 0,00 & 0,35 \\
\hline 3 & 2 & 0 & 6 & 1 & 17 & 20 & 0,07813 & 103,4 & 76,9 & 0,0 & 250,0 & 55,6 & 379,3 & 413,8 & 0,00 & 0,35 \\
\hline 2 & 3 & 0 & 6 & 1 & 17 & 20 & 0,07813 & 69,0 & 111,1 & 0,0 & 250,0 & 55,6 & 379,3 & 413,8 & 0,00 & 0,35 \\
\hline 1 & 4 & 0 & 6 & 1 & 17 & 10 & 0,03906 & 34,5 & 142,9 & 0,0 & 250,0 & 55,6 & 379,3 & 413,8 & 0,00 & 0,35 \\
\hline 0 & 5 & 0 & 6 & 1 & 17 & 2 & 0,00781 & 0,0 & 172,4 & 0,0 & 250,0 & 55,6 & 379,3 & 413,8 & 0,00 & 0,35 \\
\hline 5 & 0 & 1 & 4 & 2 & 17 & 1 & 0,00391 & 172,4 & 0,0 & 41,7 & 173,9 & 105,3 & 344,8 & 413,8 & 0,25 & 0,29 \\
\hline 4 & 1 & 1 & 4 & 2 & 17 & 5 & 0,01953 & 137,9 & 40,0 & 41,7 & 173,9 & 105,3 & 344,8 & 413,8 & 0,25 & 0,29 \\
\hline 3 & 2 & 1 & 4 & 2 & 17 & 10 & 0,03906 & 103,4 & 76,9 & 41,7 & 173,9 & 105,3 & 344,8 & 413,8 & 0,25 & 0,29 \\
\hline 2 & 3 & 1 & 4 & 2 & 17 & 10 & 0,03906 & 69,0 & 111,1 & 41,7 & 173,9 & 105,3 & 344,8 & 413,8 & 0,25 & 0,29 \\
\hline 1 & 4 & 1 & 4 & 2 & 17 & 5 & 0,01953 & 34,5 & 142,9 & 41,7 & 173,9 & 105,3 & 344,8 & 413,8 & 0,25 & 0,29 \\
\hline 0 & 5 & 1 & 4 & 2 & 17 & 1 & 0,00391 & 0,0 & 172,4 & 41,7 & 173,9 & 105,3 & 344,8 & 413,8 & 0,25 & 0,29 \\
\hline 5 & 0 & 0 & 5 & 2 & 17 & 1 & 0,00391 & 172,4 & 0,0 & 0,0 & 208,3 & 105,3 & 344,8 & 413,8 & 0,00 & 0,29 \\
\hline 4 & 1 & 0 & 5 & 2 & 17 & 5 & 0,01953 & 137,9 & 40,0 & 0,0 & 208,3 & 105,3 & 344,8 & 413,8 & 0,00 & 0,29 \\
\hline 3 & 2 & 0 & 5 & 2 & 17 & 10 & 0,03906 & 103,4 & 76,9 & 0,0 & 208,3 & 105,3 & 344,8 & 413,8 & 0,00 & 0,29 \\
\hline 2 & 3 & 0 & 5 & 2 & 17 & 10 & 0,03906 & 69,0 & 111,1 & 0,0 & 208,3 & 105,3 & 344,8 & 413,8 & 0,00 & 0,29 \\
\hline 1 & 4 & 0 & 5 & 2 & 17 & 5 & 0,01953 & 34,5 & 142,9 & 0,0 & 208,3 & 105,3 & 344,8 & 413,8 & 0,00 & 0,29 \\
\hline 0 & 5 & 0 & 5 & 2 & 17 & 1 & 0,00391 & 0,0 & 172,4 & 0,0 & 208,3 & 105,3 & 344,8 & 413,8 & 0,00 & 0,29 \\
\hline
\end{tabular}

\begin{tabular}{|c|c|c|c|c|c|c|c|c|c|}
\hline & $1 q 0$ & $4 q 1$ & $5 q 5$ & $5 q 10$ & $5 q 15$ & $15 q 0$ & $20 \mathrm{q} 0$ & D5-9/D10-14 & D5-14/D20 et + \\
\hline $\mathrm{e}^{\circ}(0)=20$ ans & 376,29 & 478,96 & 95,44 & 51,19 & 68,73 & \multirow{4}{*}{$\begin{array}{c}721,09 \\
\downarrow\end{array}$} & \multirow{4}{*}{$\begin{array}{c}740,25 \\
\downarrow\end{array}$} & \multirow{3}{*}{2,06} & \multirow[t]{3}{*}{0,17} \\
\hline $\mathrm{e}^{\circ}(0)=25$ ans & 320,4 & 362,76 & 76,89 & 42,62 & 58,44 & & & & \\
\hline $\mathrm{e}^{\circ}(0)=30$ ans & 269,8 & 269,52 & 61,03 & 35,04 & 49,15 & & & & \\
\hline $\mathrm{e}^{\circ}(0)=35$ ans & 224,32 & 195,88 & 47,62 & 28,39 & 40,8 & & & $Y$ & $y$ \\
\hline $\mathrm{e}^{\circ}(0)=40$ ans & 183,76 & 138,77 & 36,42 & 22,62 & 33,37 & 337,9 & 360,04 & 1,67 & 0,06 \\
\hline Meyzieu & 103,4 & 76,9 & 41,7 & 217,4 & 55,6 & 379,3 & 413,8 & 0,20 & 0,35 \\
\hline
\end{tabular}




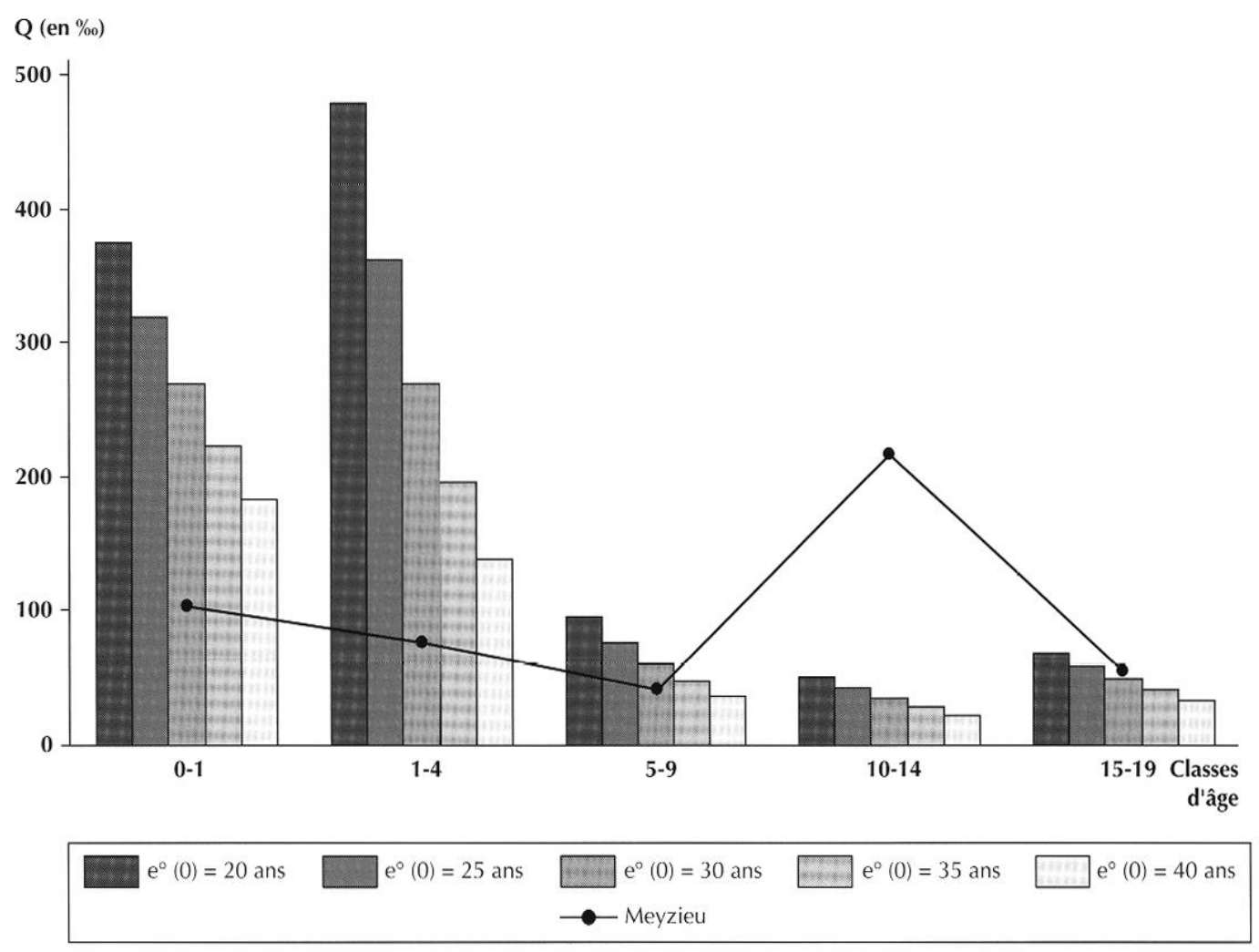

Fig. 31 - Courbe des quolients de mortalité en \%o des immatures du Trillet à Meyzieu. Confrontation avec les données des lables types de Ledermann, 1969 (graphique : D. Castex).

Tabl. VII - Les différentes possibilités de classement des sujets immatures du Pillon à Marennes. Conséquences sur les valeurs des quotients de mortalité et les rapports des effectifs des décès. Comparaison avec les tables types de Ledermann, 1969 (réalisation: D. Castex).

\begin{tabular}{|c|c|c|c|c|c|c|c|c|c|c|c|c|c|c|c|}
\hline \multicolumn{5}{|c|}{ Effectifs des classes d'âge } & \multirow{2}{*}{ Comb. } & \multirow{2}{*}{ Proba. } & \multicolumn{7}{|c|}{ Quotients de mortalité en \% } & \multicolumn{2}{|c|}{ Rapports des décès } \\
\hline $1-4$ & $5-9$ & $10-14$ & $4-$ & 20 et + & & & $1 q 0$ & $4 q 1$ & $5 q 5$ & $5 q 10$ & $5 q 15$ & $15 q 0$ & $20 \mathrm{q} 0$ & D5-9/D10-14 & D5-14/D20 et \\
\hline 0 & 3 & 1 & & 7 & 1 & 0,25000 & 0,0 & 0,0 & & & & 6 & & 300 & 0,57 \\
\hline 0 & 2 & 2 & 0 & 7 & 2 & & 0,0 & 0,0 & 31,8 & & & 363,6 & 30 & & \\
\hline 0 & 1 & 3 & 0 & 7 & 1 & 0,25000 & 0,0 & 0,0 & 90,9 & 300,0 & 0,0 & 363,6 & 363,6 & 0,33 & 0,57 \\
\hline
\end{tabular}

\begin{tabular}{|c|c|c|c|c|c|c|c|c|c|}
\hline & $1 \mathrm{q} 0$ & $4 q 1$ & $5 q 5$ & $5 q 10$ & $5 q 15$ & $15 q 0$ & $20 \mathrm{q} 0$ & D5-9/D10-14 & D5-14/D20 et + \\
\hline $\mathrm{e}^{\circ}(0)=20$ ans & 376,29 & 478,96 & 95,44 & 51,19 & 68,73 & 721,09 & 740,25 & 2,06 & 0,17 \\
\hline $\mathrm{e}^{\circ}(0)=25$ ans & 320,4 & 362,76 & 76,89 & 42,62 & 58,44 & & & & 1 \\
\hline $\mathrm{e}^{\circ}(0)=30$ ans & 269,8 & 269,52 & 61,03 & 35,04 & 49,15 & & & & \\
\hline $\mathrm{e}^{\circ}(0)=35$ ans & 224,32 & 195,88 & 47,62 & 28,39 & 40,8 & $Y$ & $y$ & $y$ & $Y$ \\
\hline $\mathrm{e}^{\circ}(0)=40$ ans & 183,76 & 138,77 & 36,42 & 22,62 & 33,37 & 337,9 & 360,04 & 1,67 & 0,06 \\
\hline
\end{tabular}

Dans le doute, nous nc pouvons que chercher à les distribuer au prorata de ce qu'ils devraient être (tabl. VI : le choix des valeurs est noté en italique; la précision est valable pour l'ensemble de cettc étude). Nous pouvons donc mettre en évidence une sous-représentation des enfants de moins de 5 ans, sans que l'on puisse trancher sur un biais exclusif des 0 an ou des $1-4$ ans. Nous pouvons toutefois préciser l'existence d'une autre anomalie, l'absence totale des périnataux, c'est-à-dire des enfants mort-nés ou décédés peu après la naissance (tous les représentants potentiels de la mortalité infantile, c'està-dire des 0 an, se situant vers le haut de cette classe). 
Fig. 32 - Courbe des quotients de morlalité en \%o des immatures du Pillon à Marennes. Confrontation. avec les données des tables types de Ledermann, 1969 (graphique: D. Castex).

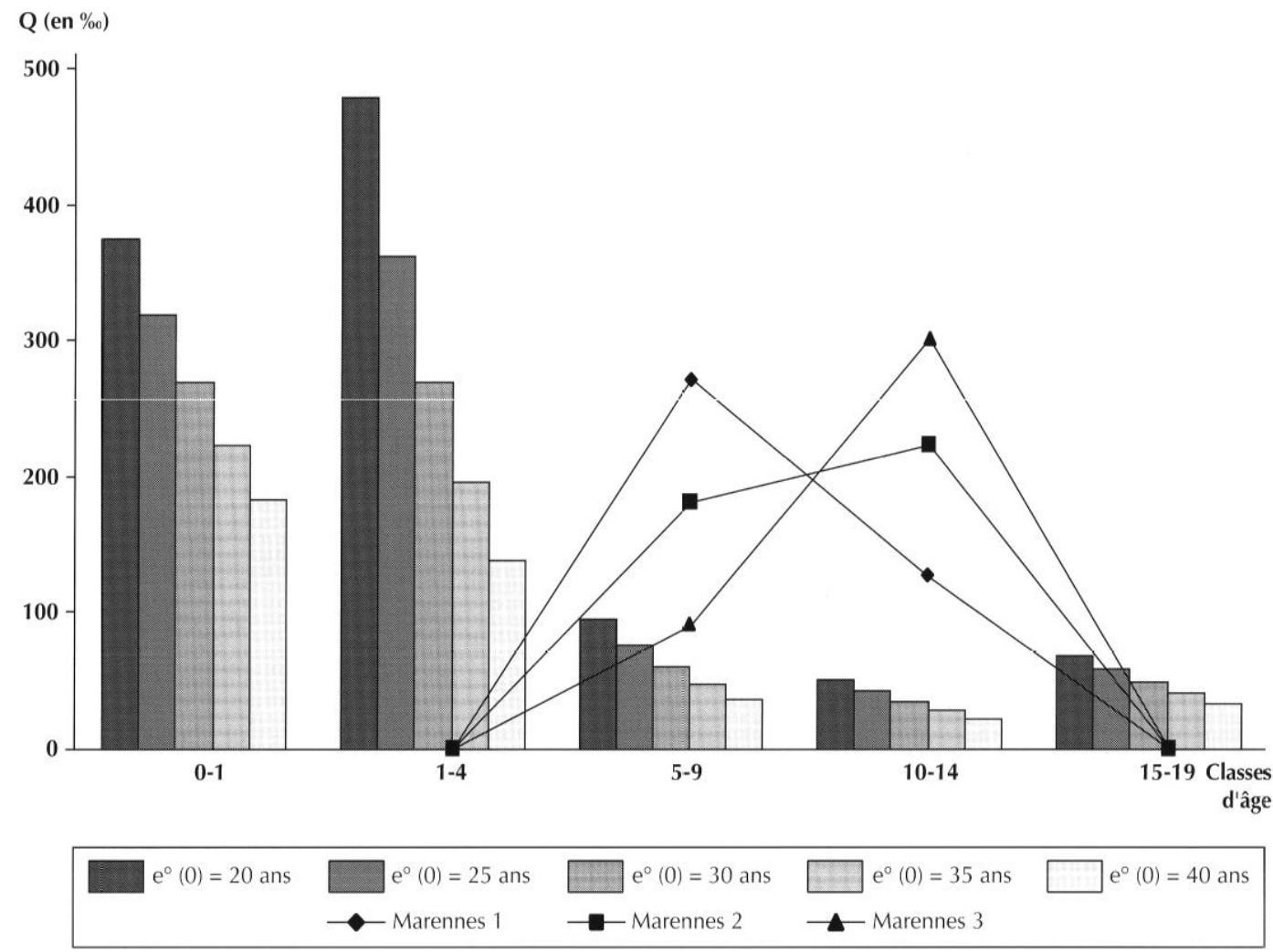

Si l'on s'intéresse maintenant aux valeurs des quotients de mortalité suivants $(5 \mathrm{Q} 5,5 \mathrm{Q} 10$ et $5 \mathrm{Q} 15)$, on remarque dans un premier temps que, quelle que soit la distribution choisie, on ne retrouve jamais la relation attendue entre ces trois classes d'âge avec un minimum situé dans la classe des 10-14 ans (Ledermann, 1969). Pour certaines distributions, les valeurs des quotients 5Q5 et 5Q15 paraissent correctes (tabl. VI), celles du quotient 5 Q10 sont toujours biaisées car beaucoup trop élevées par rapport à une mortalité archaïque. Seuls les quotients de mortalité avant 15 ans $(15 \mathrm{Q} 0)$ et avant 20 ans $\left(20 \mathrm{Q}^{0}\right)$ paraissent satisfaisants quelle que soit la distribution considćrćc (tabl. VI) ; ils prouvent unc proportion de non-adultes par rapport aux adultes tout à fait vraisemblable mais pour des espérances de vie à la naissance relativement élevées, ce qui confirme nos observations précédentes. D'autres anomalies irréductibles apparaissent: en raison du taux anormalement élevé des 10-14 ans, le rapport des $\mathrm{D}(5-9) / \mathrm{D}(10-14)$ se révèle extrêmement faible, il ne dépasse pas la valeur de 0,25 (il est proche de 2 dans le cas de populations préjennériennes) ; pour la même raison, l'indice de juvénilité $\mathrm{D}(5-14) / \mathrm{D}(20$ et +$)$ est, à l'inverse, anormalement élevé. Toutes ces données nous conduisent à privilégier une seule distribution (tabl. VI, valeurs notées en caractères gras), celle qui présente le moins d'anomalies tout en ayant une des plus fortes probabilités de faire apparaître certains critères corrects.

La courbe des quotients de mortalité obtenue pour Le Trillet et comparée aux histogrammes issus des tables types de Ledermann (1969) permet de mieux visualiser les différences (fig. 31). Nous pouvons observer que la distribution des âges au décès des sujets immatures fournit des résultats qui, malgré nos efforts pour les minimiser, présentent quelques anomalics irréductibles. La premic̀rc conccrnc la sous-représentation sélective des enfants de moins de 5 ans ; cette sous-représentation pourrait concerner les deux classes d'âge 0 an et $1-4$ ans (hypothèse privilégiée dans l'optique de minimiser les anomalies) ou bien ne concerner que la mortalité infantile, l'absence totale des périnataux étant un fait certain. Le biais exclusif des 1-4 ans paraît peu probable, étant donné que toutes les autres tranches d'âge sont représentées. Une autre anomalie est la nette disproportion des classes d'âge 5-9, 10-14 et 15-19 ans entre elles ; c'est ce qu'expri- 
ment le rapport des $\mathrm{D}(5-9) / \mathrm{D}(10-14)$ relativement bas et l'indice de juvénilité $\mathrm{D}(5-14) / \mathrm{D}(20$ et + ) désignant un effectif d'adultes anormalement faible par rapport à l'effectif des 5-14 ans. Ainsi pour Le Trillet, nous pouvons conclure à une population "non naturelle " pour les immatures.

Pour Le Pillon, le problème s'avère nettement plus simple (tabl. VII et fig. 32). Les quatre individus dont l'âge a pu être estimé ont entre 5 et 14 ans (un individu dans la classe 5-9 ans, un dans la classe 10-14 ans et les deux autres dans l'interclasse 5-9/10-14 ans). Nous obtenons donc seulement trois distributions possibles pour quatre combinaisons. Toutes font évidemment apparaître des quotients de mortalité avant 20 ans totalement biaisés. Seuls les quotients de mortalité avant 15 ans $\left(15 Q^{0}\right)$ et avant 20 ans $\left(20 Q^{0}\right)$ paraissent corrects, quelle que soit la distribution considérée (tabl. VII); ils prouvent une proportion de non-adultes par rapport aux adultes tout à fait vraisemblable mais pour des espérances de vie à la naissance relativement élevées.

\section{INTERPRÉTATION DES ENSEMBLES FUNÉRAIRES}

Les trois ensembles funéraires étudiés ici partagent certains caractères, tels que leur petit nombre de sépultures, la durée relativement courte de leur occupation et leur situation vraisemblable ou supposée dans le finage de l'habitat. Néanmoins, leur examen approfondi semble indiquer que les caractères communs généraux énoncés ci-dessus sont insuffisants pour envisager que ces ensembles présentent une organisation identique.

L'inhumation est généralisée, ce qui est sans surprise pour la seconde moitié du IV ${ }^{\mathrm{e}}$ s., les crémations les plus tardives ne dépassant pas le courant du III ${ }^{\mathrm{e}} \mathbf{s}$. en milieu rural (Bel et al., 1991, p. 16), sauf dans de rares cas (Collis, 1977). Les tombes reconnues entrent dans la typologie régionale de leurs secteurs géographiques, avec par exemple les coffres de tegulae plus courants dans la France du Sud que dans la partie nord de Rhône-Alpes. Cependant, Le Trillet ne livre aucun contenant cloué, forme pourtant typique de l'Antiquité tardive. Ce caractère pourrait trouver une justification dans l'hypothèse d'une datation un peu plus tardive de ce site par rapport aux autres. On y rencontre en tout cas une morphologie pour l'instant inconnue dans la littérature pour les périodes représentées ici, caractérisée par des contenants en matériaux périssables de plan hexagonal. Enfin, on reconnaît pour la première fois dans la moyenne vallée du Rhône des sépultures anthropomorphes antérieures à l'époque carolingienne, qui n'avaient pour l'instant été rencontrées qu'en Languedoc. L'analyse taphonomique témoigne d'une extraordinaire variabilité des types de tombes. En regard des séries que nous avons eu l'occasion d'étudier par ailleurs, cette variabilité paraît caractériser l'Antiquité de manière générale et n'apparaît pas spécifique de ces petits ensembles.

Les gestes funéraires ne sont pas exactement les mêmes sur les trois sites. Si les mutilations des céramiques sont attestées sur tous, d'après les vestiges archéologiques qui nous sont parvenus, seules les sépultures du Pillon révèlent un rituel complexe au cours duquel des récipients ont été brisés sur les contenants. Le mobilier est également plus diversifié au Pillon. Aux Girardes, les pratiques paraissent moins ostentatoires, d'autant qu'il reste difficile de savoir si les inhumations de chiens sont liées au rituel ou s'il s'agit d'animaux morts naturellement et inhumés dans l'ensemble funéraire. Les vestiges du rituel sont encore plus discrets au Trillet qui livre un cortège céramique réduit. Mais, là encore, la question d'un léger décalage chronologique peut être posée: arrivons-nous au terme d'un processus qui est amorcé depuis le $\mathrm{II}^{\mathrm{e}} \mathrm{s}$. ? Toutefois, la diminution du nombre de vases est marquée principalement par la quasi-disparition des vases à solides, probablement remplacés par de la vaisselle en bois comme l'attestent les offrandes alimentaires retrouvées directement sur le fond de la fosse.

Les trois sites retenus dans cette étude sont manifestement de nature différente.

Celui du Pillon paraît représenter l'un des modes d'occupation courant de l'Antiquité tardive, défini par l'évolution d'un " établissement » de type indigène en fonction au I ${ }^{\text {cr }} \mathrm{s} .{ }^{6}$ marquée par des reconstructions et l'édification d'un bâtiment en bois (Mahé, Séguier, 1995). Ce dernier est de plan classique avec sa nef unique, d'un type fréquent en Protohistoire (Arcelin, Buchenschutz, 1985, p. 21). Attesté au Haut-Empire, ce mode de construction ne représente en rien une innova-

6. La nature de l'occupation du Haut-Empire ne peut être définie en raison du peu de vestiges qui lui sont attribuables. On a l'impression d'une occupation de faible ampleur. 
tion (Van Ossel, 1997, p. 85). Les témoins matériels de cette occupation sont trop peu nombreux pour préciser la nature des activités qui se déroulaient dans les bâtiments. Seule la présence, à proximité du bâtiment de la parcelle $\mathrm{A}$, d'une fosse remplie de déchets de plomb fondu témoigne d'une activité métallurgique sur le site. La fonction des parcelles est inconnue sauf pour la $\mathrm{A}$ (résidentielle), mais l'hypothèse d'une partition de l'espace fondée sur des activités différentes est probable, du fait de la diversité des structures selon les parcelles et de l'absence de vestiges dans l'une d'elles. La séparation entre les fonctions économique et domestique est effective à un moment de l'histoire du site comme l'atteste la subdivision de l'espace par des fossés ( $c f$. supra, p. 276). Une réorganisation de l'espace aux II IIII $^{\mathrm{e}} \mathrm{s}$. est cependant envisageable, si l'on admet que certains alignements de trous de poteaux peuvent correspondre à des palissades. Les faits sont moins clairs à la fin de l'Antiquité où le fossé 6 par exemple est condamné. On peut se demander encore si le bâtiment de bois, qui renferme une grande fosse dans sa partie sud-ouest dont la fonction primaire est inconnue, a également été habité. Il est possible qu'on ait, d'une certaine manière, l'illustration du resserrement de l'habitat mis en évidence partout dans l'Antiquité tardive (Van Ossel, 1997), mais les données dont nous disposons sont trop indigentes pour raisonner précisément sur l'évolution topochronologique du site. Il n'en demeure pas moins que si l'ensemble funéraire est lié à cette occupation, les structures résidentielles paraissent insuffisantes. Doit-on supposer que d'autres formes de bâtiments existaient et qu'elles n'ont pas laissé de traces, le site étant relativement arasé ? On connaît en effet dans l'architecture romaine des élévations sur soles à plat et des solins très partiellement enterrés (Halbout, Le Maho, 1984, p. 18 et fig. 8). L'hypothèse évoquée d'une expansion de l'établissement à partir des II $^{c}$ III $^{\mathrm{c}} \mathrm{s}$. paraît plausible, bien que les fossés situés dans la partie est n'aient pas pu être datés puisqu'il n'existe aucun mobilier du ${ }^{\text {er }}$ s. sur le site en dehors de celui recueilli dans les fossés de l'enclos A. Le site du Pillon rappelle une série de petits établissements exhumés en région parisienne, comme celui du Chemin de Sens à Marolles-sur-Seine (Seine-et-Marne), fouillé intégralement (Mahé, Séguier, 1995). Il s'agit du même modèle d'établissement de type indigène, inscrit dans le système parcellaire antique et caractérisé par un enclos quadrangulaire de forme trapézoïdale dont l'espace interne est subdivisé à l'aide de fossés. Le Pillon s'en rapproche également par l'organisation générale de l'espace, par son architecture domestique, par une expansion dans l'Antiquité tardive et par un mobilier traduisant un niveau de vie assez aisé (habitat et ensemble funéraire). La durée de l'ensemble funéraire paraît correspondre à celle de l'occupation tardo-antique et il est créé ex nihilo, dans la mesure où aucune tombe du HautEmpire n'a été repérée aux alentours.

Aux Girardes, l'occupation paraît réduite par rapport à celle du Haut-Empire. Cependant, cette remarque doit être pondérée par le fait que tous les bâtiments mis au jour sont liés à l'exploitation agricole, aucun ne présentant des indices d'habitation. L'abandon de la vigne au début du III $\mathrm{s}$. montre que l'on passe à une monoculture (céréales), tandis que les fonctions s'élargissent à l'élevage (étable). Ainsi, l'Antiquité tardive semble caractérisée par une occupation d'un type un peu différent. Des édifices seuls quelques réaménagements $d u$ bâtiment initial nous parviennent, mais cela ne signifie pas forcément que l'installation est ponctuelle. En effet, on peut envisager, de la même manière qu'au Pillon, que la nature de l'habitat du IV $\mathrm{s}$. n'est pas compatible avec sa survie archéologique (édifices en bois sur solins par exemple). Le lieu funéraire s'est déplacé par rapport à ceux du Haut-Empire, ce qui correspond à un processus courant (Van Ossel, 1993, p. 186), et on remarque qu'il n'est pas moins étendu que ces derniers. D'après les éléments de datation dont on dispose, sa durée de vie est inférieure à celle de l'habitat.

Le cas est un peu différent au Trillet. Il existe une permanence du lieu d'habitat depuis le Haut-Empire, mais les limites de l'occupation I ${ }^{\text {er-II }}{ }^{e} s$. définies par les fossés sont clairement transgressées. L'habitat se caractérise par des structures légères dont certaines sont semi-excavées. Ces structures excavées ne peuvent en aucun cas être assimilées à des fonds de cabanes. Des constructions de ce type, dépourvues de trous de poteaux et datées du VI ${ }^{\mathrm{e}} \mathbf{s}$., ont été relevées au Recourbe à Château-Gaillard dans l'Ain, mais elles sont de taille plus réduite (Vicherd et al., 1985). Celles du Trillet sont inédites dans notre région. Elles se rapprochent plutôt, par leur taille $\left(12 \mathrm{~m}^{2}\right.$ à $84 \mathrm{~m}^{2}$ ), par l'absence, sauf dans un cas, de trous de poteau, par les vestiges de fondations légères sur le pourtour de la fosse et par le sol empierré de la structure 22, de celles de Lixhe en Belgique et de Harff I en Allemagne, datées de la seconde moitié du IV ${ }^{e}$ s., quali- 
fiées de constructions mixtes (Van Ossel, 1992, p. 127). En France, on signalera encore le site du Chemin de Sens à Marolles-sur-Seine, où deux structures de ce type sont relevées (Mahé, Séguier, 1995, p. 73-74). La fonction précise de ces structures est toujours difficile à interpréter et ce n'est qu'à l'aide du mobilier recueilli sur le site (outils, céramiques fines) que l'hypothèse d'une occupation à la fois domestique et agricole peut être proposée. Mais comme au Pillon, la distinction parmi les différentes structures ne peut être opérée. L'absence de foyer à l'intéricur de ces structures ne permet pas de distinguer, malgré leur grande taille, celles qui correspondent aux bâtiments d'habitation de celles dont la fonction pourrait être artisanale. Il semblerait que la durée de l'occupation de l'ensemble funćraire soit supérieure à celle de l'occupation domestique repérée sur la surface décapée.

Le travail réalisé par P. Van Ossel pour le nord de la Gaule révèle une profonde mutation de l'habitat rural à partir du III' $s$. Il propose l'hypothèse de la mise en place d'un système " pré-villageois » caractérisé par le regroupement d'unités socio-familiales, préfigurant les formes de l'habitat mérovingien (Van Ossel, 1992, p. 183-184). La zonc d'habitat du Trillet, malgré la vision partielle que l'on en a, scmblerait se rapporter à ce modèle. C'est d'ailleurs ce genre d'ćtablissement tout en structures légères et semi-excavées, créćes parfois ex nihilo, qui perdure jusqu'au VII' s. (Van Ossel, 1997, p. 87). Un décapage extensif autour de la zone fouillée, plus sûr que ne l'ont été les sondages effectués, aboutirait-il à la mise en évidence d'un habitat lâche et structuré où des structures plus tardives se juxtaposeraient aux vestiges tardo-antiques? Les travaux menés dans la région de Lunel-Viel par C. Raynaud et F. Favory ont révélé des processus de réorganisations successives de l'occupation, dans lesquelles, du Haut-Empire au haut Moyen Âge, l'habitat se déplace sous la forme de plusieurs noyaux qui se succèdent (Favory et al., 1994, p. 220). Le site de Bourbousson à Chabrillan dans la Drôme montre un déplacement des unités d'occupation sur quelques centaines de mètres au cours des mêmes périodes, dont celle du haut Moyen Âge livre des sépultures isolées en marge de l'habitat (Blaizot, Savino, à paraître). Pour cc qui concerne Le Pillon et Les Girardes, il reste difficile de faire la part entre ce qui relèverait du réaménagement d'un système antérieur et une toute nouvelle forme d'organisation de l'habitat. Il est cependant probable qu'aux Girardes les reprises de certains fossés, le développement de la culture céréalière et, somme toute, le fait que l'activité se maintienne dans l'emprise de l'exploitation antéricure illustrent la persistance du système. Le maintien d'un grand nombre d'établissements du Haut-Empire est d'ailleurs aujourd'hui bien attesté, parfois jusqu'aux $\mathrm{V}^{\mathrm{e}}-\mathrm{VI}^{\mathrm{e}} \mathrm{s}$. (Favory et al., 1994, p. 218). De même au Pillon, l'identification de matériel tardif dans tous les secteurs et l'apparente diversité des structures relevées laissent envisager une certaine prospérité à la fin de l'Antiquité. À la même période le développement de la villa de Saint-Fréjus située à proximité montre un processus identique, mais nous nous garderons d'amalgamer ces divers phénomènes, la persistance de la vie rurale dans les villae n'impliquant en aucun cas une continuité du réseau socioéconomique auquel elles appartenaient. La localisation des sites autour d'un pôle urbain tel que Vienne constitue peut-être l'un des éléments déterminants de cette évolution.

Les nombreux exemples attestés dans d'autres régions, comme celle de la petite Seine où ces petits établissements paraissent quasi exclusifs, la coexistence de ce mode d'occupation avec la villa et d'autres formes d'habitat permettent de relativiser l'hypothèse d'une " hiérarchie économique liée à la propriété foncière " (Mahé, Séguier, 1995, p. 83). En d'autres termes, il paraît de moins en moins probable qu'ils constituent des annexes d'ćtablissements plus importants et les trois sites retenus ici ne font qu'illustrer la variabilité des modes de l'habitat rural antique. Malheureusement, dans la plaine de Iyon et la moyenne vallée du Rhône, nous ne disposons pas pour l'instant d'un nombre représentatif de sites permettant de comprendre les relations entre ces différents types d'établissements.

Les données recueillies au Pillon ou aux Girardes et, dans une autre mesure, au Trillet rendent compte d'une évolution sans rupture des petits établissements, de la même manière que les villae. À la fin de l'Antiquité, où le mode d'exploitation de la villa est en crise, l'adaptation se traduit par une réorganisation de l'espace au sein du même finage et ce phénomène, attesté sur ces trois sites, est conforme au modèle reconnu dans d'autres régions.

Malgré leurs différences structurelles, les trois sites illustrent un fait identique et bien connu, caractérisé par la création d'un ensemble funéraire dans la seconde moitié du IV ${ }^{\mathrm{e}} \mathrm{s}$. à proximité d'un habitat dont l'occupa- 
tion est ininterrompue depuis le $\mathrm{I}^{\mathrm{er}} \mathrm{s}$. Les tombes plus anciennes se situent ailleurs.

Cependant, malgré la proximité indéniable de toutes ces tombes et des structures domestiques, il est légitime de se demander si ces ensembles funéraires correspondent systématiquement aux habitats qu'ils jouxtent. Si cela parait probable au Pillon et aux Girardes, peut-être parce que l'on dispose de l'ensemble de l'occupation, la vision tronquée que l'on a de l'habitat du Trillet, avec ses quatre structures dont deux paraissent plutôt devoir être attribuées aux III"-IV" s., conjuguée à la présence ponctuelle de sépultures plus tardives dans l'ensemble funéraire, ne permet pas de l'affirmer. Tout au plus, l'identification de mobilier des $I^{\mathrm{c}}-\mathrm{V}^{\mathrm{c}} \mathrm{s}$. témoigne d'une synchronie de l'habitat et de quelques sépultures au moins pour cette période. À cela s'ajoute la difficulté de véritablement caractériser les types d'occupation : continuité d'un établissement antérieur avec une réelle exploitation, réoccupation ponctuelle sommaire, présence sporadique, etc. Peut-être cédons-nous généralement trop vite au modèle " exploitation = nécropole = unité familiale ", et il convient alors de réfléchir plus précisément à l'organisation de ces ensembles funéraires.

À cet égard, l'examen du "recrutement " de ces ensembles est riche d'informations. Au Pillon, la répartition par sexes et par âges de la population inhumée est compatible avec l'hypothèse de familles nucléaires, à condition bien sûr d'interpréter les biais observés sur les deux premières classes d'âge comme représentatifs des ensembles funéraires gallo-romains en général et non spécifiques des nôtres. Or, ce constat parait tout à fait acceptable, la sous-représentation des jeunes enfants étant largement mise en évidence ailleurs pour presque chaque étude de nécropole tardo-antique, qu'il s'agisse d'un petit ou d'un grand ensemble funéraire. Par ailleurs, les multiples découvertes de sépultures du Haut et du Bas-Empire de très jeunes enfants en contexte d'habitat (Struck, 1993; Duday et al., 1995), voire d'ensembles funéraires constitués uniquement d'individus décédés au début de leur vie (site de Pourliat à Beaumont dans le Puy-de-Dôme, cf. Alix, Blaizot, 2000 ; site de La Ramière à Roquemaure dans le Gard, cf. Blaizot, 1997), paraissent confirmer que ces petits enfants ne sont pas inhumés avec les adultes. L'hypothèse de familles nucléaires est alors envisageable ici. En effet, malgré un faible effectif, la répartition par sexe est conforme à celle d'une population homogène et la proportion des immatures sur l'ensemble de l'effectif inhumé est correcte.

En revanche, au Trillet, les caractères anormaux du " recrutement " concernent en tout premier lieu la distribution par sexe des individus adultes avec une très forte majorité d'hommes. Une autre anomalie est illustrée par le déficit des jeunes adultes (moins de 30 ans). Enfin une dernière discordance, et non des moindres, est celle de la disproportion des classes d'âge des sujets immatures (biais important des moins de 5 ans et sureffectif des 10-14 ans). Ainsi, l'ensemble funéraire du Trillet accueille des individus sélectionnés et non pas la totalité de la population décédée, et cette sélection va au-delà du système en vigueur dans le monde antique qui consiste à écarter les jeunes enfants, puisqu'il manque une partie des adultes: les femmes et les jeunes. Le recours aux données paléopathologiques et à celles des caractères discrets, insatisfaisantes en raison de leur faible fréquence, de la conservation des ossements mais aussi de problèmes méthodologiques, ne nous autorise pas, pour l'instant, à argumenter sur les raisons d'une telle sélection au Trillet. De plus, l'impossibilité d'attribuer chaque tombe à une phase chronologique précise peut nous conduire à interpréter de manière erronée ce qui pourrait n'être qu'un changement au cours du temps du mode de "recrutement ". Il n'est pas possible de définir si les modalités particulières du " recrutement " valent pour toute la durée de l'ensemble funéraire ou si elles n'ont été que momentanées. Nous ne pouvons pas prouver en effet que l'incohérence démographique résulte de la seule présence de sépultures du haut Moyen Âge. D'ailleurs, en contexte rural, l'usage d'inhumer de manière intermittente sur les terres existe encore au BasEmpire, en témoignent les découvertes de groupes de 5 à 10 tombes (Monnier, 2001), de tombes isolées (Van Ossel, 1993; Gébara, Pasqualini, 1993) et les tombes de La Ramière à Roquemaure (Blaizot, 1997). Il est clair que dans sa durée cet ensemble funéraire n'est pas à « recrutement » familial stricto sensu, ce qui n'exclut pas que les sujets puissent être apparentés. En ce sens, les ensembles du Pillon et du Trillet présentent une différence fondamentale. Il est dommage que l'état de la série des Girardes ne permette pas de discuter les caractéristiques de son " recrutement".

On peut s'interroger sur les raisons de la bipartition des ensembles funéraires mise en évidence au Pillon et 
aux Girardes, dans l'hypothèse admise au départ d'un ensemble funéraire à « recrutement " de type familial. Doit-on envisager deux groupes familiaux ? On a en tout cas deux unités qui fonctionnent selon le même système, dont certains éléments sont utilisés pour les distinguer l'une de l'autre. Il n'est pas exclu que l'on ait affaire dans chaque cas à deux groupes dont le niveau socio-économique est différent, l'un possédant un mobilier nombreux et diversifié et ayant des pratiques quasi ostentatoires (bris de céramiques). Mais cette explication qui paraît peut-être trop facilement tomber sous le sens reste difficile à démontrer. Par ailleurs, il n'est pas possible non plus d'exclure ni de prouver l'hypothèse d'un léger décalage chronologique à l'origine de la raréfaction voire de l'absence de mobilier dans les deux autres groupes du Pillon et des Girardes, mais en l'état de la recherche, les datations fournies par les céramiques tardives à revêtement argileux ne permettent pas d'atteindre ce degré de précision.

La taille des ensembles funéraires des Girardes et du Pillon ainsi que la chronologie fournie par la céramique indiquent qu'ils ont fonctionné sur une courte durée. Or, ces deux sites ont été décapés sur une surface très importante et aucun autre groupe de tombes tardoantiques n'a été relevé. Ce caractère est-il lié aux avatars de l'histoire de ces établissements? Cela peut être proposé pour Le Pillon, mais dans le cas des Girardes, où l'on a les traces même ténues d'une fréquentation aux $\mathrm{VI}^{\mathrm{c}}-\mathrm{VII}^{\mathrm{c}}$ s., il est clair que le lieu d'inhumation s'est déplacé, voire qu'il a changé de nature (par exemple intégration dans un grand ensemble communautaire ?). Encore une fois, le site du Trillet se distingue de ces derniers puisque sous son uniformité apparente il ne s'agit pas d'un ensemble homogène. Non seulement il livre des sépultures du haut Moyen Âge, mais son « recrutement " ne saurait correspondre à celui d'une ou de plusieurs familles; la relation entre les espaces domestiques et les espaces funéraires n'est peut-être alors pas toujours aussi bi-univoque qu'elle n'y paraît. D'autres zones situées dans le finage de l'habitat peuvent avoir été utilisées conjointement à un ensemble funéraire communautaire, et il existe d'ailleurs dans le finage de certains habitats du haut Moyen Âge des noyaux composés d'un très petit nombre de sépultures dont le " recrutement » semble aléatoire ; ils sont implantés en marge des espaces domestiques (Blaizot, Savino, à paraître). Au Trillet, il paraît bien que cet espace ait été ponctuellement fréquenté sur une longue durée pour inhumer certains individus sélectionnés dans la population décédée, soit dès le Bas-Empire, soit plus tardivement. Ainsi, si Le Pillon et Les Girardes peuvent traduire le modèle d'une permanence de l'organisation familiale antique, Le Trillet rompt avec cette tradition, en tout cas si on le considère sur l'ensemble de sa durée.

Ces petits ensembles funéraires étant ainsi caractérisés, on remarque que les systèmes mis en évidence, tant au Trillet qu'aux Girardes, ne représentent pas quelque chose de véritablement nouveau par rapport à ce qui se passe au Haut-Empire, où les espaces funéraires ruraux apparaissent très diversifiés (ensembles familiaux, tombes ou groupes de tombes dispersés). Plus précisément, les petits ensembles présentés ici ne témoignent ni d'une réorganisation des espaces funéraires ni d'une modification des relations spatiales entre l'habitat et les lieux dévolus aux morts au sein du finage. Concernant l'organisation des ensembles du Pillon et des Girardes, on ne paraît pas s'écarter du modèle des petits groupes communautaires du Haut-Empire dont on ne peut que supposer la nature familiale, tandis que les pratiques funéraires observées se placent dans la continuité des traditions de la période précédente. Les modalités du " recrutement » du Trillet montrent, quant à elles, que le système de la multiplicité des espaces funéraires propre au Haut-Empire est encore effectif, puisque cet ensemble ne saurait renfermer une communauté complète. Il convient également de relativiser la notion de discontinuité qui paraît caractériser les ensembles du Haut et du Bas-Empire, fréquemment attestée bien que non systématique. Peut-on en effet interpréter l'émergence de nouveaux groupes de tombes au IV $\mathbf{s}$. comme l'illustration d'un déplacement des espaces funéraires marquant une rupture consentie, dans la mesure où s'il peut exister un lieu bien défini pour les morts au Haut-Fmpire il est rare qu'il soit utilisé sur une. longue durée?

Ces remarques nous invitent à ne pas assimiler de manière péremptoire les diverses formes d'habitat aux différents modes funéraires. En d'autres termes, il paraît de plus en plus certain que toute modification du système socio-économique est loin d'entraîner instantanément une réorganisation des espaces funéraires. Le monde des morts est d'une certaine manière un groupe à part, avec ses propres modes d'organisation et le poids des traditions peut s'avérer important. On se trouve là 
encore probablement dans le même mode d'occupation qu'au Haut-Empire, défini par des petits espaces sépulcraux utilisés sur une courte durée et implantés dans les zones non exploitées des établissements. Cependant, si l'ensemble du Trillet entre dans le modèle de la multiplicité des espaces funéraires du Haut-Empire puisqu'il n'a pu exister seul, il s'en écarte par le fait qu'il perdure. Le lieu sépulcral, bien que fréquenté ponctuellement, semble fixé en tant que tel. Ce site illustre un système particulier qui résulte sans doute de l'histoire de son occupation.

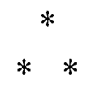

Ces trois petits ensembles montrent combien il est difficile de caractériser l'occupation funéraire en contexte rural et d'en dégager des traits qui lui soient propres. Il en ressort que parallèlement à quelques caractères communs, une certaine variabilité existe dans l'organisation et le rituel funéraires.

Le problème majeur de l'interprétation des phénomènes mis en évidence ici est général à l'archéologie ; il réside principalement dans notre difficulté à aborder la synchronie des différents ensembles comparés, comme celle des sépultures et de l'habitat. Bien entendu, cette question de la synchronie nous renvoie à celle de la dimension diachronique des traits dégagés. Les pratiques funéraires observées d'un site à l'autre sont-elles significatives d'une différence entre les groupes ou sontelles liées aux paramètres chronologiques et donc aux changements?

On peut émettre le postulat que les trois sites renferment un nombre suffisamment représentatif de sépultures qui sont synchrones. Dans ce cas, par rapport aux tombes du Trillet pouvant être attribuées au Bas-Empire, la diversité, l'abondance et le traitement du mobilier de celles du Pillon pourraient traduire une différence de statut entre ces sites. Si l'on en croit les modestes vestiges que livrent Le Pillon et Les Girardes, ce caractère ne paraît cependant pas marqué à travers ce qui nous est parvenu de l'habitat, mais la question de la survie archéologique de ce dernier reste posée. Quelques groupes témoigneraient en tout cas d'une organisation sociale plus complexe qu'il n'y paraît, comme semble le montrer la présence de deux ensembles spatiaux au Pillon et aux Girardes, dont les attributs sont différents. Ces différences pourraient également correspondre à une plus importante fidélité aux traditions gallo- romaines selon les groupes, qui conduirait certains à exprimer leur statut à travers les pratiques funéraires. Les gestes reconnus, tels que les mutilations de vases et les bris de vaisselle, directement hérités du Haut-Empire, sont incontestablement compatibles avec l'hypothèse d'un certain traditionalisme. Cependant, ce caractère traditionnel n'est pas particulier à ces petits ensembles. En effet, les mutilations de vases sont rencontrées dans tous les contextes et, parmi les grands ensembles, certains attestent même de rituels peu fréquents tels des foyers élevés au sommet du comblement des fosses (Blaizot, à paraitre).

La variabilité des pratiques funéraires évoquée cidessus ne semble pas relever d'une spécificité rurale des sépultures. Une quasi égale diversité est en effet observée dans les villes, malgré un fonds commun propre aux contextes urbains (plus grande rareté du mobilier, utilisation plus fréquente de la pierre dans l'architecture funéraire). Celle-ci peut être illustrée par trois sites pris au hasard à Lyon : à Saint-Just sur la colline (contexte basilical, cf. Reynaud, 1998), au quai Arloing à Vaise, et à la clinique Saint-Charles à La Croix-Rousse (contextes périurbains, cf. Tranoy, Gisclon, 1995 et fouille inédite de D. Frascone et F. Blaizot). Aucun de ces trois sites n'ayant fait l'objet de décapages suffisamment extensifs pour qu'il soit possible d'analyser l'organisation de chaque ensemble, la variabilité est observée uniquement dans les modes de dépôts. Chacun livre des architectures funéraires très diverses. Bien que Saint-Just illustre un contexte basilical, les dépôts de mobilier ne sont pas absents et on a également des traces de repas funéraires (Reynaud, 1998, p. 205); à la clinique Saint-Charles, le mobilier est réduit à sa plus simple expression de dépôts de vases dans quelques tombes, tandis qu'au quai Arloing, ces dépôts dont des récipients mutilés, nombreux à la transition III $-\mathrm{IV}^{\mathrm{e}} \mathrm{s}$., disparaissent totalement au début du $\mathrm{IV}^{\mathrm{e}} \mathrm{s}$. (Tranoy, Gisclon, 1995, p. 221).

La diversité des modes funéraires doit également être envisagée du point de vue de l'organisation des ensembles sépulcraux puisque l'étude du "recrutement'» des trois sites montre qu'ils sont de nature différente. L'un se caractérise par l'accueil ponctuel de sépultures sur une longue durée (Le Trillet) et les deux autres abritent tous les membres d'une petite communauté à l'exclusion des très jeunes enfants. Ils correspondent à deux systèmes que l'on rencontre jusqu'à la fin du haut Moyen Âge 
dans le monde rural ${ }^{7}$, parallèlement à ces grands ensembles communautaires dont le « recrutement" dépasse incontestablement le cadre du groupe familial.

En cette fin de l'Antiquité, que les récents travaux menés dans le sud et le nord de la France permettent aujourd'hui de qualifier de période de transition et non pas de rupture (Raynaud, 1996 ; Van Ossel, 1997), les vestiges archéologiques rendent compte d'une grande variabilité qui s'inscrit dans un cadre cohérent. Variabilité des formes d'habitat et des modes funéraires, continuité et discontinuité qui coexistent dans unc époque de mutations sociale, économique et culturelle. Dans le finage des petits ćtablissements on n'observe aucune réorganisation fondamentale des espaces sépulcraux. Les pratiques funéraires et les lieux d'inhumation sont loin d'être figés et l'analyse des petits ensembles ruraux des périodes qui suivent montre qu'ils ne le seront pas avant longtemps (Blaizot, Savino, à paraître).

7. Les tout petits cnsembles communautaires peuvent être illustrés pour le VI"s. par le site de Dassargues près de Montpellicr (Garnotel, Raynaud, 1996) et pour les VII'-XI' s. par les sites de Saint-Martin à Chabrillan et de Saint-Romain à Espeluche dans la Drôme (Blaizot, Savino, à paraître). Des exemples de très petits groupes de sépultures dispersés aux confins de l'habitat sont mentionnés dans les communications présentées au colloque des XIV" Journées internationales d'archéologie mérovingienne de Guiry-en-Vexin en 1993 (Lorren, Périn, 1995), et une étude de cas a ćté récemment réalisée pour des sites de la moyenne valléc du Rhône (Blaizot, Savino, à parâtre).

\section{Remerciements}

Nous remercions Jean-Pierre Daugas, Conservateur régional de l'archéologie, de nous avoir accordé un financement sur les crédits "Sauvetages urgents régionaux » et les responsables d'opération de nous avoir autorisés à publier les sites. De même, nous sommes reconnaissants envers l'AFAN-Rhône-Alpes-Auvergne d'avoir financé la numérisation de l'ensemble des figures.

Nos remerciements s'adressent également à Benoît Helly (SRA, Lyon), Claude Raynaud (UMR 154 du CNRS) et tout particulièrement Paul Van Ossel (UMR $7041 \mathrm{du}$ CNRS) qui nous ont conseillés pour la rédaction définitive de ce manuscrit.

Armand Desbat, Directeur du laboratoire de céramologie de Lyon, a eu l'amabilité d'aider à l'identification des dépôts en céramique.

Merci à Marina Biron, AFAN-Bordeaux (nettoyage des monnaies) ; à Jacqueline Cathalaa, laboratoire d'anthropologie de Bordeaux I (relevés des sépultures du Trillet) ; à Maryelle Bessou, laboratoire d'anthropologie de Bordeaux I (radiographie des objets); à Jean-Marc Degueule, musée de la Civilisation gallo-romaine de Lyon (photos des céramiques); à Catherine Plantevin, AFAN-Lyon (dessin du tonnelet en céramique des Girardes). 


\title{
INVENTAIRE DESCRIPTIF DES SÉPULTURES
}

\author{
Rappel des caractéristiques principales des sépultures : mode d'inhumation, estimation sexe et âge au décès, \\ association et situation du mobilier \\ Mode d'inhumation : F. Blaizot, H. Duday. \\ Estimation sexe et âge au décès : F. Blaizot, D. Castex. \\ Identification des céramiques : $\mathrm{C}$. Bonnet. \\ Identification des monnaies : C. Cécillon, D. Frascone. \\ Identification du mobilier métallique : M. Rolland, M. Feugère (Les Girardes).
}

\section{LE TRILLET À MEYZIEU}

\section{SÉPULTURE SP1}

Mode d'inhumation : indéterminé.

Adulte de sexe indéterminé. Tête au nord-est. Avant-bras ramenés en avant de l'abdomen, mains sur le pelvis.

Mobilier céramique (pl. $\left.\mathrm{V}, \mathrm{n}^{\circ} 1\right): 1$ cruche proche de Des. 84, revêtement argileux; située à gauche du crâne.

\section{SÉPULTURE SP2}

Mode d'inhumation: dépôt du corps à même la fosse dont le profil transversal est en gouttière ; couverture en matériau périssable aménageant un espace vide.

Immature : classe 10-14 ans. Tête au nord-nord-est. Avant-bras en extension, le droit parallèle à l'axe longitudinal du corps, le gauche sur le pelvis.

Mobilier céramique (pl. $\left.\mathrm{V}, \mathrm{n}^{0} \mathrm{l}\right): 1$ pot ansé en céramique commune grise, placé latéralement au genou droit.

Mobilier métallique (pl. V, $\mathrm{n}^{\text {os }} 2-6$ ) :

- 5 bracelets fermés en bronze identiques : décor d'incisions verticales régulières sur l'ensemble. Diamètre intérieur $60 \mathrm{~mm}$, hauteur $2 \mathrm{~mm}$, épaisseur $1 \mathrm{~mm}$. En avant de l'hémi-thorax gauche.

- 1 perle polyédrique en verre bleu (hauteur $8 \mathrm{~mm}$, largeur $7 \mathrm{~mm}$ ), fragment d'anneau en bronze, crochet fermé en bronze : boucle d'oreille (?) située contre le temporal gauche. Matériel perdu, non dessiné.

Monnaie: une monnaie de bronze de petit module (aes 4), placée en avant de la tête fémorale gauche (fig. 22, $\mathrm{n}^{\circ} 7$ ).

Il peut s'agir d'une imitation radiée qui fut fabriquée en masse dans des ateliers locaux de contrefaçon à la fin du
III' $\mathrm{s}$. après J.-C. Ces petites monnaies ont circulé au IV ${ }^{\mathrm{c}} \mathrm{s}$.

D) Illisible.

Tête radiée à droite.

R) Illisible.

\section{SÉPULTURE SP3}

Mode d'inhumation : dépôt du corps à même la fosse ? Décomposition du corps en espace vide.

Immature : classe 10-14 ans. Tête au nord-est. Avant-bras en extension, mains en avant des os coxaux.

\section{SÉPULTURE SP4}

Squelette non conservé. Quelques éléments du bloc cranio-facial au sud.

\section{SÉPULTURE SP5}

Mode d'inhumation : dépôt du corps à même la fosse dont le profil transversal est en gouttière ; décomposition en espace vide.

Adulte féminin. Tête au sud-est. Avant-bras en extension, mains en avant du tiers proximal des fémurs.

\section{SÉPULTURE SP6}

Mode d'inhumation : contenant rectangulaire complet, en bois non cloué, relativement étroit.

Immature : classe 10-14 ans. Tête au nord-est. Avant-bras en extension, le gauche parallèle à l'axe longitudinal du corps, la main droite en arrière de l'os coxal homolatéral.

Mobilier métallique : 18 clous, 18 fragments en fer (hauteur $14 \mathrm{~mm}$, largeur $9 \mathrm{~mm}$ ) à tête quadrangu- 
laire, pointe courte tordue. Clous de chaussures. Non dessinés.

\section{SÉPULTURE SP7}

Mode d'inhumation : contenant rectangulaire complet, en bois non cloué.

Immature : classes 10-14/15-19 ans. Tête au nord-est. Avant-bras gauche en extension avec la main en avant du tiers proximal de la cuisse, le droit ramené en avant de la moitié gauche de l'abdomen.

\section{SÉPULTURE SP8}

Mode d'inhumation : contenant de forme indéterminée, en bois non cloué.

Adulte masculin. Tête au sud-est. Avant-bras en extension avec les mains en avant du tiers proximal de la cuisse.

\section{SÉPULTURE SP9}

Mode d'inhumation : dépôt du corps à même la fosse dont le profil transversal est en gouttière ; décomposition en espace vide aménagé par une couverture maintenue sur des banquettes latérales par des pierres.

Adulte masculin. Tête au nord-ouest. Avant-bras gauche ramené sur le pelvis, le droit ramené en avant du thorax. Mobilier métallique : 2 pointes de clous en fer (hauteur $30 \mathrm{~mm}$ ) ; les têtes ont disparu, l'usage est donc inconnu mais il ne s'agit pas de clous de chaussures. La hampe de section quadrangulaire mesure $8 \mathrm{~mm} \times 5 \mathrm{~mm}$ de largeur. Situées près de la paroi sud de la fosse. Non dessinées.

\section{SÉPULTURE SP10}

Mode d'inhumation: contenant de forme hexagonale, en bois non cloué.

Adulte masculin. Tête au nord-est. Avant-bras ramenés en avant de l'abdomen, mains sur le pelvis.

Mobilier céramique (pl. V, ${ }^{\circ} 1$ ) : 1 gobelet P. 62 var. 130/ Lam. 14/26, revêtement argileux. Situé à droite du crâne.

\section{SÉPULTURE SP11}

Mode d'inhumation : contenant de forme indéterminée, en bois non cloué.

Adulte masculin ? Tête au nord-est. Avant-bras gauche en extension avec la main en avant du tiers proximal de la cuisse, le droit ramené sur le pelvis.

Mobilier céramique (pl. V, $\left.\mathrm{n}^{\circ} 1\right): 1$ petite cruche, revêtement argileux ; située latéralement au pied droit.

\section{SÉPULTURE SP12}

Mode d'inhumation : coffrage rectangulaire, en bois non cloué; couvercle seul cloué sur la cuve; absence de plancher.

Adulte masculin. Tête au nord-est. Avant-bras droit en extension avec la main en avant du tiers proximal de la cuisse, le gauche ramené en avant de l'abdomen, main sur le pelvis.

Mobilier métallique : 1 pointe de clou en fer : la tête très lacunaire semble plate, la hampe est de section quadrangulaire (largeur $5 \mathrm{~mm}$, hauteur $42 \mathrm{~mm}$ ). L'usage reste inconnu, mais il ne s'agit pas de clou de chaussure. Située latéralement à l'extrémité distale de la fibula gauche. Non dessinée.

\section{SÉPULTURE SP13}

Mode d'inhumation : coffrage rectangulaire, en bois non cloué ; absence de plancher.

Adulte masculin. Tête au nord-ouest. Avant-bras gauche en extension parallèle à l'axe longitudinal du corps, le droit ramené en avant de l'abdomen, main sur le pelvis.

\section{SÉPULTURE SP14}

Mode d'inhumation : indéterminé.

Adulte (?) de sexe indéterminé. Tête au nord-est.

\section{SÉPULTURE SP15}

Mode d'inhumation: réceptacle du corps étroit de nature indéterminée ; absence de fond construit.

Immature : classe 1-4 ans. Tête au nord-est.

Mobilier céramique (pl. V, $\mathrm{n}^{\circ} 1$ ) : 1 cruche Des. 85/P. 95, revêtement argileux. Située latéralement à la jambe droite.

\section{SÉPULTURE SP16}

Mode d'inhumation : cercueil rectangulaire, en bois non cloué ; plancher constitué de deux planches. 
Adulte féminin. Tête au nord-est. Avant-bras ramenés sur le pelvis.

Mobilier céramique (pl. $\left.\mathrm{V}, \mathrm{n}^{\circ} \quad 2\right): 1$ gobelet P. 62/Lam. 14/26. Situé à droite du crâne.

Mobilier métallique : 13 clous de chaussures en fer en six fragments. Hampe courte, pointue et tordue, tête quadrangulaire (hauteur $11 \mathrm{~mm}$, largeur $7 \mathrm{~mm}$ ). Situés au pied gauche. 5 clous en fer du même type au pied droit. Il est important de noter le très petit gabarit de ces clous. Non dessinés.

Dépôt alimentaire (fig. 10, $n^{\circ} 1$ ) : ossements de volatile latéralement à droite du crâne sur le fond de la fosse.

\section{SÉPULTURE SP17}

Mode d'inhumation : contenant de forme trapézoïdale, en bois non cloué.

Adulte masculin. Tête au nord-est. Avant-bras ramenés en avant de l'abdomen.

\section{SÉPULTURE SP18}

Mode d'inhumation : cercueil de forme hexagonale, en bois non cloué ; plancher constitué de deux planches.

Adulte féminin? Tête au nord-est. Avant-bras en extension, parallèles à l'axe longitudinal du corps.

\section{SÉPULTURE SP19}

Mode d'inhumation : contenant de forme hexagonale probable, en bois non cloué.

Adulte masculin? Tête au nord-est. Avant-bras en extension, parallèles à l'axe longitudinal du corps.

\section{SÉPULTURE SP20}

Mode d'inhumation : contenant de forme hexagonale, en bois non cloué.

Adulte masculin. Tête au nord-ouest. Avant-bras droit en extension parallèle à l'axe longitudinal du corps, le gauche ramené en avant de l'abdomen.

\section{SÉPULTURE SP21}

Mode d'inhumation : contenant de forme indéterminée, en bois non cloué.

Adulte masculin. Tête au nord-est. Avant-bras droit en extension parallèle à l'axe longitudinal du corps, le gauche ramené en avant de l'abdomen.

\section{SÉPULTURE SP22}

Mode d'inhumation : contenant rectangulaire, en bois non cloué.

Adulte féminin jeune. Tête au nord-est. Avant-bras ramené en avant de l'abdomen, mains sur le pelvis.

\section{SÉPULTURE SP23}

Mode d'inhumation : squelette non conservé. Quelques fragments de crâne au nord-ouest.

\section{SÉPULTURE SP24}

Mode d'inhumation : contenant de forme indéterminée, en bois non cloué ; absence de plancher.

Adulte de sexe indéterminé. Tête au nord-est. Avant-bras ramenés en avant de l'abdomen, mains sur le pelvis.

\section{SÉPULTURE SP25}

Mode d'inhumation: sépulture détruite. Quelques éléments de crâne au nord-ouest.

\section{SÉPULTURE SP26}

Mode d'inhumation : contenant de forme indéterminée, en bois non cloué ; absence de plancher.

Immature : classes 5-9/10-14 ans. Tête au nord-est. Attitude des avant-bras indéterminée.

\section{SÉPULTURE SP27}

Mode d'inhumation : contenant de forme trapézoïdale, en bois non cloué ; absence de plancher.

Immature : classe 10-14 ans. Tête au nord-est. Avant-bras ramenés en avant de l'abdomen, mains sur le pelvis.

Mobilier métallique ( $\left.\mathrm{pl} . \mathrm{V}, \mathrm{n}^{\circ} 1\right): 1$ boucle en fer de section circulaire, située latéralement à l'extrémité proximale du fémur droit. Le porte-ardillon est droit, le cintre en arc outrepassé. L'ardillon de section rectangulaire s'amincit vers la pointe légèrement recourbée. Il est fixé autour du porte-ardillon par un simple enroulement. 


\section{SÉPULTURE SP28}

Mode d'inhumation : contenant de forme indéterminée, en bois non cloué.

Immature : classes 0/1-4 ans. Tête au nord-ouest. Mains sur le pelvis.

\section{SÉPULTURE SP29}

Mode d'inhumation : contenant de forme trapézoïdale, en bois non cloué ; absence de plancher.

Adulte féminin. Tête au nord-ouest. Avant-bras ramenés en avant de l'abdomen, le gauche en situation proximale par rapport au droit.

\section{SÉPULTURE SP30}

Mode d'inhumation : espace vide de nature indéterminée.

Immature : classes 0/1-4 ans. Tête au nord-est. Attitude des avant-bras indéterminée.

Monnaie: une monnaie de bronze de petit module (aes 4).

$\mathrm{n}^{\circ} 23$ située dans l'hémi-thorax droit.

Imitation radiée de la fin du III's. $s$ début du IV $\mathrm{e}$ s. après J.-C. ?

D) Illisible.

Tête radiée à droite.

R) Illisible.

\section{SÉPULTURE SP31}

Mode d'inhumation : contenant de forme trapézoïdale, cn bois non clouć ; présence d'un plancher.

Immature : classe 10-14 ans. Tête au nord-est. Avant-bras en extension, parallèles à l'axe longitudinal du corps.

\section{SÉPULTURE SP32}

Mode d'inhumation : espace vide de nature indéterminée.

Immature : classes 0/1-4 ans. Tête au nord-est. Attitude des avant-bras indéterminée.

\section{LE PILLON À MARENNES}

\section{SÉPULTURES SP502/503}

Ces sépultures ont été détruites au décapage, on n'a aucune indication sur le mode d'inhumation ni sur la provenance exacte et la localisation des objets.

$\mathbf{2}$ adultes de sexe indéterminé. Orientation inconnue.

Mobilier métallique (pl. I, $\mathrm{n}^{\text {os }} 1$ et 2 ) :

- 1 bracelet en bronze $\left(\mathrm{n}^{\circ} 1\right)$ complet, en deux fragments. Ruban large avec une extrémité large, arrondie et perforée, l'autre amincie recourbée en crochet (longueur $183 \mathrm{~mm}$, largeur 2-10 mm, épaisseur $1 \mathrm{~mm}$ ). Décor : deux bandeaux d'incisions obliques régulières encadrent une frise comportant une incision en vague et des cupules. L'état de surface ne permet pas d'observer si cette frise est régulière. Un tiers de la surface du bracelet est caché par les restes ou l'empreinte d'un textile tissé: restes du vêtement? Une bossette en saillie marque la fin du registre décoratif avant le crochet et la perforation du système d'attache.

- 1 bracelet en bronze $\left(n^{\circ} 2\right)$ complet, déformé par écrasement. Ruban épais avec une extrémité légèrement plus large perforée et l'autre étroite avec un crochet légèrement aplati pour une meilleure sécurité (longueur $183 \mathrm{~mm}$, largeur 4-6 mm, épaisseur 1-2 mm). Décor régulier d'incisions en chevrons.

- 4 clous en fer. Hampe de section quadrangulaire $(6 \mathrm{~mm} \times 6 \mathrm{~mm})$, tête plate circulaire (diamètre $22 \mathrm{~mm}$ ), longueur approximative de la pièce $90 \mathrm{~mm}$. Non dessinés.

- 1 clou en fer. Hampe de section quadrangulaire $(7 \mathrm{~mm}$ x $7 \mathrm{~mm}$ ), tête aplatie et trapézoïdale (largeur $22 \mathrm{~mm}$, épaisseur $4 \mathrm{~mm}$ ), longueur approximative de la pièce : $115 \mathrm{~mm}$. Non dessiné.

Monnaie : $\mathrm{n}^{\circ} 12$, maiorina de Magnence (350-353).

D) DN MAGN[ENT]IVS PF AVG

Buste nu-tête, drapé et cuirassé, à droite de Magnence.

R) SALVS DD N[N AVG] ET [C]AES

Ex) Lettres non visibles/Dans le champ : $\mathrm{S}$ à gauche et * à droite.

Grand chrisme accosté de l'alpha et de l'oméga (A et $\Omega$ ). Poids 5,90 g (flan court mais épais); diamètre $20 \mathrm{~mm}$; axe $12 \mathrm{~h}$; bronze.

Atelier : Lyon?

Émission : 353 après J.C.

Réf. : non attribuable sans la marque d'atelier. 


\section{SÉPULTURE SP504}

Mode d'inhumation: contenant rectangulaire, en bois non cloué.

Immature : classe 10-14 ans. Tête au sud. Avant-bras ramenés en avant du thorax, le droit en situation proximale par rapport au gauche.

Mobilier céramique (pl. II, $\mathrm{n}^{\text {os }} 1$ et 2) $: 1$ cruche non identifiée, revêtement argileux, très fragmentaire, non dessinée (fig. $30, \mathrm{n}^{\circ}$ 50) ; 1 gobelet $\mathrm{P}$. 66, revêtement argileux $\left(n^{\circ} 2\right) ; 1$ plat $P$. 1 , revêtement argileux $\left(n^{\circ} 1\right)$. Ces deux récipients sont placés latéralement au fémur gauche.

Mobilier métallique (pl. II) :

- 1 maillon en forme de « 8 » en bronze accroché à un petit anneau en fer (diamètre extérieur $9 \mathrm{~mm}$ ) d'un côté et à une chaîne de trois petits anneaux en fer de l'autre (non dessiné) ; 2 anneaux (fig. 30, n 54) en fer, cassés et présentant une concrétion ferreuse à leur jonction (non dessinés) ; 6 maillons de chaînette $\left(n^{\circ} 12\right)$ en bronze en forme de « 8 ", de section semi-circulaire (chaque maillon $20 \mathrm{~mm} \times 9 \mathrm{~mm} \times 3 \mathrm{~mm}$, longueur totale conservée : $112 \mathrm{~mm}$ ) ; deux maillons en forme de « 8 » en bronze avec corrosion ferreuse (non dessinés); 12 fragments : conglomérats d'anneaux circulaires formant une chainette avec deux maillons en forme de « 8 » en bronze (non dessinés, fig. $30, \mathrm{n}^{\circ} 56$ ) ; 1 plaque en forme de lancette $\left(n^{\circ} 6\right)$, en bronze avec une extrémité en crochet et l'autre en anneau dont la fin de la pièce est en ruban enroulé. Une corrosion ferreuse contenant un anneau en fer y est accrochée : cette pièce fonctionnait peut-être avec les autres maillons en bronze et les anneaux en fer.

Toutes ces pièces semblent former un ensemble : peutêtre une ceinture. Elles se trouvaient dans un coffret en matière périssable.

- 1 maillon en forme de " 8 " $\left(\mathrm{n}^{\circ} 4\right)$ en bronze $17 \mathrm{~mm}$ x 6-7 mm x $1 \mathrm{~mm} ; 1$ maillon en forme de « 8 » $\left(\mathrm{n}^{\circ} 9\right)$ en bronze. Ces deux pièces sont identiques. Leurs dimensions sont très différentes de celles de l'ensemble précédent. Situées au niveau de la poitrine.

- 14 clous de chaussures en fer avec tête quadrangulaire épaisse et hampe courte (hauteur $10 \mathrm{~mm}$ ), placés au pied droit (fig. 30, $\mathrm{n}^{\circ} 79$, non dessinés).

- 4 clous en fer $\left(\mathrm{n}^{\circ} 10\right)$ avec hampe de section quadrangulaire très pointue et à tête plate quadrangulaire (hauteur $21 \mathrm{~mm}$, largeur et longueur $8 \mathrm{~mm}$ ). Usage indé- terminé. Placés dans le coffret en matière périssable.

- 1 lampe en fer $\left(\mathrm{n}^{\circ} 8\right)$. Vasque: hauteur $20 \mathrm{~mm}$, diamètre intérieur 70-75 mm très légèrement ovalaire, épaisseur $2 \mathrm{~mm}$. Manche : vertical, très lacunaire, avec un rétrécissement à la jointure (largeur $16 \mathrm{~mm}$ ), conservé sur une hauteur de $24 \mathrm{~mm}$, il part en s'évasant (largeur maximale $29 \mathrm{~mm}$, épaisseur $2 \mathrm{~mm}$ ). La vasque et le manche ne forment qu'une seule pièce. Placée dans le coffret en matière périssable.

- 1 lot de perles en verre $\left(n^{\circ} 7\right): 1$ «cylindrique » verte, de section hexagonale (hauteur $5 \mathrm{~mm}$, diamètre $4 \mathrm{~mm}$ ) ; 1 cylindrique verte (hauteur $5 \mathrm{~mm}$, diamètre $3 \mathrm{~mm}$ ); 3 ellipses beiges (hauteur $3 \mathrm{~mm}$, diamètre $5 \mathrm{~mm}$ ); 1 ellipse verte (hauteur $3 \mathrm{~mm}$, diamètre $5 \mathrm{~mm}$ ); 86 ellipses bleu sombre (hauteur $1-4 \mathrm{~mm}$, diamètre 4-7 mm). L'ensemble placé en avant de la poitrine et de l'abdomen pourrait former un collier d'une longueur totale minimale de $344 \mathrm{~mm}$.

- 1 perle en verre $\left(n^{\circ} 11\right)$ située en amont et à gauche du crâne. Quasi sphérique, légèrement aplatie en haut et en bas (hauteur $9 \mathrm{~mm}$, diamètre $11 \mathrm{~mm}$ ). Décor: trois incisions latérales circulaires se chevauchent, créant six panneaux ornés chacun d'une incrustation circulaire jaune.

- 1 anneau cassé ( $\left.\mathrm{n}^{\circ} 13\right)$ constitué d'un ruban ou de deux rubans torsadés (longueur conservée $76 \mathrm{~mm}$, épaisseur 3-4 mm). La situation de cet objet, en amont et à gauche du crâne, nous conduit à l'identifier comme une boucle d'oreille plutôt que comme une bague.

- 1 bracelet torsadé $\left(n^{\circ} 3\right)$, en bronze, complet (trois fragments), porté à l'avant-bras droit. Formé d'un fil de cuivre régulièrement torsadé, une extrémité en anneau cassée, l'autre en crochet, renflement à l'opposé de l'attache (diamètre intéricur 47-53 mm, épaisseur 4-7 mm). - 1 bracelet en bronze $\left(\mathrm{n}^{\circ} 5 \mathrm{a}\right)$, complet en trois fragments, porté à l'avant-bras gauche. Ruban avec une extrémité quadrangulaire légèrement élargie et une perforation circulaire, l'autre relevée en crochet avec bouton quadrangulaire pour retenir et accrocher (longueur $166 \mathrm{~mm}$, largeur 6-7 mm, épaisseur $1 \mathrm{~mm}$ ). Décor : deux rangées de cupules longent les bords tandis que le bandeau central présente des incisions verticales très proches, en vague. Le bord de l'extrémité perforée présente deux incisions décoratives en queue d'aronde.

- 1 bracelet en bronze $\left(\mathrm{n}^{\circ} 5 \mathrm{~b}\right)$ porté à l'avant-bras gauche. Ruban fin avec une extrémité en lancette, perfo- 
ration rectangulaire, l'autre extrémité, disparue, comportait sans doute un crochet. Les quatre fragments permettent de reconstituer une longueur de $168 \mathrm{~mm}$, largeur $45 \mathrm{~mm}$, épaisseur $1 \mathrm{~mm}$. Décor: l'état de la surface ne laisse voir correctement que deux rangées de cupules longeant les bords du ruban. Le bandeau central semble porter des incisions, mais elles sont impossibles à déchiffrer.

- 1 bracelet en bronze ( $\left.\mathrm{n}^{\circ} 5 \mathrm{c}\right)$ porté à l'avant-bras gauche. Ruban de section semi-sphérique, une extrémité perforée cassée, l'autre en crochet, renflé pour une meilleure accroche (longueur conservée $188 \mathrm{~mm}$ ). Décor: sur la moitié face au système d'attache, cinq groupes formés d'incisions larges obliques " affrontées " encadrant deux rangées de quatre ocelles autour de quatre incisions simples rayonnantes. Le reste de la surface comporte de simples incisions verticales régulièrement espacées. Avant le crochet et la perforation, une saillie quadrangulaire évoque la bossette du modèle de la sépulture SP502/503.

Dépôt alimentaire : 1 volatile complet situé en amont des céramiques (fig. $30, \mathrm{n}^{\circ} 90$ ).

\section{SÉPULTURE SP505}

Mode d'inhumation: contenant rectangulaire, en bois non cloué. Il reposait au sud sur un imbrex, tandis qu'au nord le fond de la fosse remonte de manière à atteindre le niveau de la face supérieure de l'imbrex placé à l'autre extrémité.

Immature : classes 5-9/10-14 ans. Tête au sud. Attitude des avant-bras indéterminée.

Mobilier céramique (pl. I, $\mathrm{n}^{\text {os }} 1-3$ ) : 1 gobelet $\mathrm{P}$. 76 à revêtement argileux aux pieds $\left(\mathrm{n}^{\circ} 1\right) ; 1$ coupe carénée en céramique commune rouge, placée en avant de la jambe gauche $\left(\mathrm{n}^{\circ} 2\right) ; 1$ pot à lèvre en amande en céramique commune grise, situé latéralement au pied droit $\left(n^{\circ} 3\right)$.

Monnaies :

Deux monnaies, situées latéralement au membre inférieur droit.

- $\mathrm{n}^{\circ}$ 6, follis (1/96 de livre) de Constantin I (307-337).

D) IMP CONSTANTINVS AVG

Buste lauré et cuirassé, à droite de Constantin.

R) SOLI INVICTO COMITI

Ex) PLG/Dans le champ : TF à gauche et * à droite.

Le soleil debout à gauche, tenant un globe et levant la main droite.
Poids $2,90 \mathrm{~g}$; diamètre $20 \mathrm{~mm}$; axe $12 \mathrm{~h}$; bronze.

Atelier: Lyon ( $1^{\text {re }}$ officine).

Émission : 315 après J.-C.

Réf. : RIC 34, Bastien $596 f$.

- $\mathrm{n}^{\circ}$ 7, follis (1/96 de livre) de Constantin I (307-337).

D) IMP CONSTANTINVS AVG

Buste lauré et cuirassé, à droite de Constantin.

R) SOLI INVICTO COMITI

Ex) PLG/Dans le champ : TF à gauche et * à droite.

Le soleil debout à gauche, tenant un globe et levant la main droite.

Poids $2,80 \mathrm{~g}$; diamètre $20,2 \mathrm{~mm}$; axe $11 \mathrm{~h}$; bronze.

Atelier : Lyon ( $1^{\text {re }}$ officine)

Émission : 315 après J.-C.

Réf. : RIC 34, Bastien 596e.

Quatre monnaies empilées dans le pot en céramique commune grise :

- $\mathrm{n}^{\circ} 8$, follis (1/72 de livre) de Constantin I (307-337).

D) CONSTANTINVS PF AVG

Buste lauré et cuirassé, à droite de Constantin.

R) SOLI INVICTO COM[ITI]

Ex) PLG/Dans le champ : $\mathrm{S}$ à gauche et $\mathrm{F}$ à droite.

Le soleil à demi-nu, debout à gauche, tenant un globe et levant la main droite.

Poids $4,90 \mathrm{~g}$; diamètre $22,2 \mathrm{~mm}$; axe $6 \mathrm{~h}$; bronze

Atelier : Lyon ( $1^{\text {re }}$ officine).

Émission : 312 après J.-C.

Réf. : RIC 7, Bastien 544c.

- $\mathrm{n}^{\circ}$ 9, follis (1/96 de livre) de Constantin I (307-337).

D) IMP CONSTANTINVS AVG

Buste lauré et cuirassé, à droite de Constantin.

R) SOLI INVICTO COMITI

Ex) PLG/Dans le champ : $\mathrm{T}$ à gauche et $\mathrm{F}$ à droite.

Le soleil radié à demi-nu, debout à gauche, tenant un globe et levant la main droite.

Poids $3,4 \mathrm{~g}$; diamètre $20,3 \mathrm{~mm}$; axe $6 \mathrm{~h}$; bronze.

Atelier : Lyon ( $1^{\text {re }}$ officine).

Émission : 315 après J.-C.

Réf. : RIC 20, Bastien 566.

- $\mathrm{n}^{\circ} 10$, follis (1/96 de livre) de Constantin I (307-337).

D) IMP CONSTANTINVS AVG

Buste lauré et cuirassé, à droite de Constantin.

R) SO[LI] IN [VIC]TO COMITI

Ex) PLG/Dans le champ : TF à gauche et * à droite.

Le soleil radié à demi-nu, debout à gauche, tenant un globe et levant la main droite.

Poids $2,8 \mathrm{~g}$; diamètre $19,6 \mathrm{~mm}$; axe $6 \mathrm{~h}$; bronze. 
Atelier : Lyon ( $1^{\text {re }}$ officine).

Émission : 315 après J.-C.

Réf. : RIC 34, Bastien 596.

- $\mathrm{n}^{\circ} 11$, follis (1/96 de livre) de Licinivs I (308-324).

D) IMP L[ICI]NIVS PF [AVG]

Buste lauré et cuirassé (?), à droite de Licinius.

R) [G]ENIO POP ROM

Ex) Illisible/Dans le champ : $\mathrm{T}$ à gauche et $\mathrm{F}$ à droite.

Le Génie du peuple romain à demi-nu, debout à gauche, coiffé du modius, tenant une patère et une corne d'abondance.

Poids $3,5 \mathrm{~g}$; diamètre $21,8 \mathrm{~mm}$; axe $12 \mathrm{~h}$; bronze.

Atelier : Lyon?

Émission : 315 après J.-C.

Réf. : Bastien 572 ou 573 ?

\section{SÉPULTURE SP506}

Mode d'inhumation : indéterminé.

Immature : âge indéterminé. Tête au nord. Attitude des avant-bras indéterminée.

Mobilier céramique (pl. I, nos 1 et 2 ) : 1 coupe $P$. var. 24 à revêtement argileux, placée aux pieds $\left(n^{\circ} 1\right) ; 1$ cruche ou 1 gobelet à revêtement argileux, située à droite de la tête $\left(n^{\circ} 2\right)$.

\section{SÉPULTURE SP507}

Mode d'inhumation: cercueil rectangulaire, en bois cloué.

Immature : classe 5-14 ans. Tête au sud. Avant-bras ramenés en avant de la poitrine, le gauche en situation proximale par rapport au droit.

Mobilier céramique (pl. III, $\mathrm{n}^{\text {os }} 1$ et 2) : 1 gobelet $\mathrm{P} .66$ à revêtement argileux $\left(\mathrm{n}^{\circ} 1\right) ; 1$ plat $\mathrm{P} .1$ à revêtement argileux placé aux pieds $\left(\mathrm{n}^{\circ} 2\right) ; 4$ fragments d'un vase à revêtement argileux appartenant à la sépulture SP508.

Mobilier métallique (pl. III, $\mathrm{n}^{\text {os }}$ 3-5) :

- 1 lampe en fer ( $n^{\circ} 8$, non dessinée). L'objet, éclaté en une multitude de fragments, n'est pas archéologiquement complet, son identification est donc douteuse. Deux fragments plus importants nous restituent une ébauche de sa forme originale. Il se trouve en avant de la diaphyse fémorale gauche.

- 8 clous et 4 fragments en fer. Hampe quadrangulaire longue et pointue, tête verticale, plate et trapézoïdale (longueur $100 \mathrm{~mm}$, largeur $23 \mathrm{~mm}$, épaisseur $6 \mathrm{~mm}$ ).
Situés autour du squelette, les clous appartiennent au cercueil (non dessinés).

- 1 bracelet $\left(n^{\circ} 4\right)$ en bronze. Section semi-circulaire s'amincissant aux extrémités, l'une relevée en crochet simple, l'autre cassée au milieu d'une perforation circulaire (longueur $180 \mathrm{~mm}$, largeur 1-3 mm, épaisseur 1-3 mm). L'état de surface ne permet pas de lire un décor. Porté à l'avant-bras gauche.

- 1 bracelet $\left(n^{\circ} 5\right)$ en bronze. Section semi-circulaire, une extrémité en crochet simple, l'autre en palette plate et large avec petite perforation circulaire (longueur $166 \mathrm{~mm}$, largeur 3-7 mm, épaisseur 1-3 mm). Décor : la moitié opposée à l'attache présente une alternance d'incisions obliques et d'ocelles, le reste est occupé par des incisions larges régulièrement espacées ; deux saillies quadrangulaires avec un ocelle les séparent du crochet et de la palette perforée. Porté à l'avant-bras droit.

- 1 bracelet $\left(n^{\circ} 3\right)$ en bronze. Section en arc outrepassé s'amincissant fortement, aux extrémités en pointe. Pas de fixation (longueur $169 \mathrm{~mm}$, largeur 2-5 mm, épaisseur 1-4 mm). L'état de surface ne laisse apparaître aucun décor. Porté à l'avant-bras gauche.

\section{SÉPULTURE SP508}

Mode d'inhumation : contenant rectangulaire, en bois non cloué ; présence d'un support en matériau périssable sous le crâne.

Adulte féminin ? Tête au nord. Avant-bras en extension, le gauche parallèle à l'axe longitudinal du corps, la main droite sur le pelvis.

Mobilier céramique (pl. I, $\mathrm{n}^{\text {os }} 1-3$ ) $: 1$ cruche non identifiée à revêtement argileux, placée aux pieds $\left(n^{\circ} 1\right)$; 1 gobelet $\mathrm{P} .65$ à revêtement argileux en avant de l'épaule gauche $\left(\mathrm{n}^{\circ} 2\right) ; 1$ plat en céramique commune grise, placé aux pieds $\left(n^{\circ} 3\right)$.

Mobilier métallique : 9 clous de chaussures, 5 têtes de clous en fer, situés aux pieds (non dessinés). Hampe courte et droite (type sépulture 504), tête quadrangulaire.

\section{SÉPULTURE SP509}

Mode d'inhumation: cercueil rectangulaire, en bois cloué.

Adulte masculin. Tête au nord-nord-ouest. Avant-bras 
droit ramené sur le pelvis, le gauche en extension parallèle à l'axe longitudinal du corps.

Mobilier céramique (pl. III, $\mathrm{n}^{\circ \mathrm{s}} 1$ et 2) : 1 gobelet P. 66 à revêtement argilcux, placé à droite du crâne $\left(n^{\circ} 1\right)$; 1 plat Lam. 4/36-P. 7 à revêtement argileux, à l'origine brisé sur le contenant et dont les fragments sont dispersés $\left(n^{\circ} 2\right)$.

Mobilier métallique (pl. III, $\mathrm{n}^{\circ} 3$ ) : 11 clous appartenant au cercueil $\left(n^{\circ} 3\right)$ et 4 fragments en fer. Tête plate quadrangulaire, hampe longue de section quadrangulaire (longueur $17 \mathrm{~mm}$, largeur $8 \mathrm{~mm}$, épaisseur 2-6 mm).

\section{SÉPULTURE SP510}

Mode d'inhumation : indéterminé.

Immature : classe 5-9 ans. Tête au nord. Attitude des avant-bras indéterminée.

Mobilier céramique (pl. III, $\mathrm{n}^{\circ} 1$ ) : 1 gobclet Lam. 14/26 à revêtement argileux, situé à gauche du crâne.

Mobilier métallique (pl. III, $\mathrm{n}^{\circ}$ 2) : 1 anneau en bronze, placé au niveau de la jambe droite. Section circulaire (diamètre intéricur $19 \mathrm{~mm}$, diamètre extérieur $27 \mathrm{~mm}$ ).

\section{SÉPULTURE SP511}

Mode d'inhumation : indéterminć.

Stade de maturité indéterminé. Tête au sud. Attitude des avant-bras indéterminée.

Mobilier céramique (pl. IV, $\mathrm{n}^{\text {os }} 1$ et 2$): 1$ gobelet P. 66 à revêtement argileux $\left(\mathrm{n}^{\circ} \mathrm{l}\right)$; l plat à lc̀vre éversé en céramique commune, non identifić, placé aux pieds $\left(n^{\circ} 2\right)$.

\section{SÉPULTURE SP512}

Mode d'inhumation : indéterminé.

Adulte de sexe indéterminé. Tête au sud. Mains sur le pelvis.

Mobilier céramique (pl. $\left.\mathrm{N}, \mathrm{n}^{\circ} 1\right): 1$ plat $\mathrm{P} .20$ à revêtement argileux, placé aux pieds.

Mobilier métallique : 1 clou de chaussure (type SP504) en fer, situé au niveau des pieds (non dessiné).

Monnaie : $\mathrm{n}^{\circ}$ 2, demi-aes 2 de Maxime (383-388).

Au niveau de la cheville gauche.

D) [DN MAX]-IMVS [PF AVG] (césure inhabituelle de MAX-IMVS, plus généralement MAXI-MVS

Buste diadémé, drapé et cuirassé (?), à droite de Maxime.

\section{R) [REPARATIO RE]IPVB}

Ex) Lettres d'atelier illisibles.

L'cmpereur debout à gauche, en habit militairc, tenant une victoriola et relevant une femme agenouilléc.

Poids $1,9 \mathrm{~g}$ (monnaie qui semble avoir été volontaircment coupée en deux dans le but de créer une divisionnairc) ; diamètre $23 \mathrm{~mm}$; axe $7 \mathrm{~h}$; bronze.

Atelier : indéterminé.

Émission : entre 383 et 388 après J.-C.

Réf. : non attribuable sans la marque d'atelier.

\section{SÉPULTURE SP513}

Mode d'inhumation : contenant rectangulaire, en bois non cloué.

Adulte féminin ? Tête au sud. Avant-bras gauche ramené en amont sur la poitrinc, lc droit indéterminé.

Mobilier céramique (pl. IV, $n^{\text {os }} 1$ et 2$): 1$ gobelet P. 75 à revêtement argileux aux pieds $\left(\mathrm{n}^{\circ} 1\right) ; 1$ coupelle $P .30$ à revêtement argileux $\left(\mathrm{n}^{\circ} 2\right)$.

Mobilier métallique : 1 fragment de bronze se présentant sous la forme d'une plaque assez fine mais ne scmblant pas correspondre à une monnaie (non dessiné). Situé en aval du genou droit.

Monnaie : $n^{\circ} 5$, débris d'une monnaie d'un module aes 2 (maiorina?) du milieu ou de la seconde moitié du IVes. après J.-C.

Au niveau présumé de l'os coxal gauche détruit.

\section{SÉPULTURE SP514}

Mode d'inhumation: contenant rectangulaire, en bois non cloué ; posé à l'origine sur des cales en matière périssable.

Adulte masculin. Tête au sud. Attitude des avant-bras indéterminée.

Mobilier céramique (pl. IV, $\left.\mathrm{n}^{\circ s} 1-3\right): 1$ plat à panse oblique en céramique commune grise $\left(\mathrm{n}^{\circ} 1\right) ; 1$ pot à lèvre en bourrelet en céramique commune grise, placé aux pieds $\left(\mathrm{n}^{\circ} 2\right) ; 1$ coupelle P. 33 à revêtement argilcux $\left(n^{\circ} 3\right)$.

\section{SÉPULTURE SP515}

Mode d'inhumation : contenant rectangulaire, en bois non cloué ; posé à l'origine sur quatre cales constituées d'imbrices et de tegulae. 
Adulte féminin. Tête au nord. Attitude des avant-bras indéterminée.

Mobilier céramique (pl. IV, $\mathrm{n}^{\text {(s }} 1$ et 2$): 1$ cruche à bec pincé, en céramique commune rouge, placé latéralement à gauche du crâne $\left(n^{\circ} 1\right) ; 1$ plat Hayes $50 \mathrm{~b}$ en sigillée africaine $\mathrm{C}$, situé aux pieds $\left(\mathrm{n}^{\circ} 2\right)$.

Mobilier métallique: 18 clous de chaussures et 6 fragments en fer trouvés aux pieds. Tête quadrangulaire épaisse, pointe quadrangulaire plus longue que dans la sépulture SP504 et systématiquement courbée (longueur actuclle $17 \mathrm{~mm}$, largeur $9 \mathrm{~mm}$ ). Non dessinés.

\section{SÉPULTURE SP516}

Mode d'inhumation : indéterminé.

Immature : âge indéterminé. Tête au nord. Attitude des avant-bras indéterminée.

Mobilier céramique (pl. IV, $\left.n^{\circ} 1\right)$ : quelques fragments d'un plat à lèvre en bourrelet, en céramique commune rouge, placés aux pieds.

\section{Monnaies :}

Trois monnaies recueillies en dehors des sépultures (niveaux d'occupation).

US 200

- $\mathrm{n}^{0} 1$, maiorina de Magnence (350-353) ou de Décence (351-353) ?

D) Légende illisible.

Buste tête nue, drapé et cuirassé (?), à droite de Magnence ou de Décence.

R) [SALVS DD NN AVG ET CAES]

Ex) Illisible.

Grand chrisme accosté de l'alpha et de l'oméga (A et $\Omega$ ).

Poids $5,4 \mathrm{~g}$; diamètre $26 \mathrm{~mm}$; axe ? ; bronze.

Atelier indéterminé.

Émission : 353 après J.-C.

Réf.: non attribuable sans la titulature et les lettres d'atelier.

US 617

- $\mathrm{n}^{\circ} 3$, minimus: imitation (?) d'un prototype d'une monnaie du IV $\mathrm{V}^{\mathrm{c}} \mathrm{s}$. ? dont la production a été massive dans les années 340-348 (Depeyrot, 1987, p. 46).

D) Illisible.

R) Illisible.

Poids $0,1 \mathrm{~g}$; diamètre $7,5 \mathrm{~mm}$; axe ?; bronze.

- $\mathrm{n}^{\circ} 4$, nummus de la dynastie valentinienne (364-378).

D) Illisible (très profonde corrosion).
Tête à droite diadémée ?

\section{R) [GLORIA ROMANORVM ?]}

Ex) Illisible.

L'empereur debout à gauche, tenant un labarum dans la main gauche et empoignant de la main droite les cheveux d'un captif agenouillé.

Poids ? (flan corrodé et cassé) ; diamètre ? (flan cassé) ; axe $1 \mathrm{~h}$; bronze.

Atelier indéterminé.

Réf.: non attribuable sans la titulature et la marque d'atelier.

\section{LES GIRARDES À LAPALUD}

\section{SÉPULTURE SP5001}

Mode d'inhumation : bâtière de tegulae. Squelette non conservé.

Mobilier céramique (pl. VI, $\mathrm{n}^{\circ} 1$ ) : 1 tonnelet en pâte calcaire $\left(\mathrm{n}^{\circ} 1\right)$; 1 plat en céramique commune grise, perdu avant le dessin. Situés à l'est dans la fosse.

\section{SÉPULTURE SP5002}

Mode d'inhumation: bâtière de tegulae. Présence à l'origine d'un support en matière périssable sous la tête et sous la partie supérieure du thorax.

Adulte féminin. Tête à l'ouest. Avant-bras ramené en avant de l'abdomen. Mains sur le pelvis.

Mobilier céramique (pl. VI, $n^{\text {*s } 1-3) ~: ~} 1$ cruche Des. 84 à revêtement argileux $\left(\mathrm{n}^{\circ} 1\right) ; 1$ pot en céramique commune grise kaolinitique $\left(n^{\circ} 3\right)$; placés latéralement au membre inférieur gauche; 1 coupe-marmite carénée en céramique commune rouge $\left(\mathrm{n}^{\circ} 2\right)$, placée latéralement au membre inférieur droit.

Monnaie : une monnaie retrouvée parmi les vertèbres cervicales disloquées (fig. $20, n^{\circ} 4$ ),

$n^{\circ} 13$, nummus de Constant (337-350).

D) CO[NSTANT] PF AVG

Buste diadémé, drapé et cuirassé, à droite de Constant.

R) [GLO]RI-A EXERCITVS

Ex) Lettres d'atelier non visibles (flan court).

Deux soldats debout se regardant, tenant chacun une haste et s'appuyant sur un bouclier; entre eux, un vexillum sur lequel est inscrit $G$.

Poids $1,2 \mathrm{~g}$; diamètre $14,5 \mathrm{~mm}$; axe $5 \mathrm{~h}$; bronze. 
Atelier : Arles.

Émission : entre 340 et 341 après J.-C.

Réf. $R I C$ non attribuable sans les lettres d'atelier, Ferrando $n^{\circ} 1038$ ou 1039.

Trois monnaies empilées près du centre de la fosse :

- $\mathrm{n}^{\circ} 14$, double maiorina de Magnence (350-353).

D) [DN MAGNEN]-TIVS PF AVG

Buste nu-tête, drapé et cuirassé, à droite de Magnence.

R) [SALVS DD NN AVG ET CAES]

Ex) Lettres d'atelier hors du champ de l'exergue (frappe décentrée vers le bas).

Grand chrisme accosté de l'alpha et de l'oméga (A et $\Omega$ ). Poids $5,6 \mathrm{~g}$; diamètre $26,6 \mathrm{~mm}$; axe $1 \mathrm{~h}$; bronze.

Atelier indéterminable sans les lettres d'atelier non venues à la frappe (Arles ou Lyon ?).

Émission : 353 après J.-C.

Réf. : non attribuable sans les lettres d'atelier.

- $\mathrm{n}^{\circ} 15$, maiorina de Décence (351-353).

D) DN DECENT[IVS CAESAR]

Buste nu-tête et cuirassé, à droite de Décence.

R) $[S A] L V[S$ DD N]N AV[G ET CAES]

Ex) $[\ldots] \mathrm{A}$ ?[...]

Poids $5,5 \mathrm{~g}$; diamètre $23 \mathrm{~mm}$; axe $12 \mathrm{~h}$; bronze.

Atelier : Arles ?

Émission : 353 après J.-C.

Réf. : non attribuable sans les lettres d'atelier.

- $\mathrm{n}^{\circ} 16$, maiorina de Constance II (337-361).

D) [DN CONSTANTIVS PF AVG]

Buste diadémé, drapé et cuirassé, à droite de Constance II.

R) FEL TEMP RE[PARATI]O

Ex) $\bullet T[R S$ ?] B dans le champ ?

L'empereur debout à gauche, en habit militaire, un bouclier sur le bras gauche, perçant de sa haste un barbare sassanide désarçonné ; à terre, un bouclier.

Poids 2,6 g (flan ébréché) ; diamètre $23,1 \mathrm{~mm}$; axe $7 \mathrm{~h}$; bronze.

Atelier : Trèves ?

Émission: 346 à 361 (mais plutôt avant réforme pondérale 353 après J.-C.).

Réf. : non attribuable sans le libellé complet des lettres d'atelier.

\section{SÉPULTURE SP5007}

Mode d'inhumation : indéterminé

Squelette non conservé.

\section{SÉPULTURE SP5008}

Mode d'inhumation : indéterminé.

Squelette non conservé.

Mobilier céramique (pl. VII, $\mathrm{n}^{\mathrm{ns}} 1$ et 2 ) :

1 cruche $\left(n^{2} 1\right)$ non identifiée à revêtement argileux ou en céramique commune claire; 1 coupe-marmite en céramique commune rouge au niveau présumé des pieds dans le quart est de la fosse $\left(n^{\circ} 2\right)$.

\section{Monnaies :}

Cinq monnaies empilées à côté de la cruche $\mathrm{n}^{\circ} 1$. Elles reposent sur le fond de la fosse.

- $\mathrm{n}^{\circ} 17$, double (?) maiorina de Magnence (350-353) ou de Décence (351-353).

D) $[\mathrm{DN} . ..] \mathrm{N}[\mathrm{TI}] \mathrm{V}[\mathrm{S} . .$.

(DN MAGNENTIVS ou DN DECENTIVS ?)

Buste nu-tête, cuirassé, à droite de Magnence ou de Décence.

R) [SALVS DD NN AVG ET CAES]

Ex) $M$ ? PL[G ?]

Grand chrisme accosté de l'alpha et de l'oméga (A et $\Omega$ ).

Poids $6,40 \mathrm{~g}$; diamètre $23,2 \mathrm{~mm}$; axe $5 \mathrm{~h}$; bronze.

Atelier : Lyon?

Émission : 353 après J.-C.

Réf. : non attribuable sans le libellé complet de la titulature et des lettres d'atelier.

- $\mathrm{n}^{\circ} 18$, double maiorina de Décence (351-353).

D) [DN DE] CENTI-[V]S CAES[AR]

Buste nu-tête, drapé (?) et cuirassé, à droite de Décence.R) [SALVS DD NN AVG ET CAES]

Ex) Lettres d'atelier hors du champ de la monnaie en raison d'une frappe décentrée.Grand chrisme accosté de l'alpha et de l'oméga (A et $\Omega$ ).

Poids $5,70 \mathrm{~g}$; diamètre $24,3 \mathrm{~mm}$; axe $12 \mathrm{~h}$; bronze.

Atelier non déterminé.

Émission : 353 après J.-C.

Réf. : non attribuable sans les lettres d'atelier.

- $\mathrm{n}^{\circ} 19$, double (?) maiorina de Magnence (350-353) ou de Décence (351-353).

D) Légende illisible (flan court).

Buste nu-tête, drapé (?) et cuirassé, à droite de Magnence ou de Décence.

R) [S]ALVS DD NN [AVG ET CAES]

Ex) Lettres d'atelier hors du champ de l'exergue.

Poids $6,80 \mathrm{~g}$; diamètre $22,3 \mathrm{~mm}$; axe $11 \mathrm{~h}$; bronze.

Atelier indéterminé (Arles ou Lyon?).

Émission : 353 après J.-C. 
Réf. : non attribuable sans la titulature complète et les lettres d'atelier.

- $\mathrm{n}^{\circ}$ 20, maiorina de Magnence (350-353).

D) [DN] MAGNEN-TIV[S] PF AV[G]

Buste nu-tête, drapé (?) et cuirassé, à droite de Magnence.

R) SALVS DD N[N AVG ET] CAE[S]

Ex) PARGrand chrisme accosté de l'alpha et de l'oméga (A et $\Omega$ ).

Poids $2,9 \mathrm{~g}$; diamètre $21,2 \mathrm{~mm}$; axe $5 \mathrm{~h}$; bronze.

Atelier : Arles (1 ${ }^{\text {re }}$ officine).

Émission : 353 après J.-C.

Ferrando $\mathrm{n}^{\circ} 1125$.

- $\mathrm{n}^{\circ} 21$, maiorina de Magnence (350-353) ou de Décence (351-353).

D) [DN...]ENT[IVS] [ PF AVG ? ou CAESAR ?]

Buste nu-tête, drapé (?) et cuirassé, à droite de Magnence ou de Décence.

R) SALVS [DD NN AVG ET CAE]S

Ex) PARGrand chrisme accosté de l'alpha et de l'oméga (A et $\Omega$ ).

Poids $2,5 \mathrm{~g}$; diamètre $20,5 \mathrm{~mm}$; axe $5 \mathrm{~h}$; bronze.

Atelier: Arles ( $1^{\text {re }}$ officine).

Réf. : non attribuable sans la titulature complète au droit.

\section{SÉPULTURE SP5021}

Mode d'inhumation : indéterminé.

Squelette non conservé. Petite fosse (longueur $0,96 \mathrm{~m}$ ) : immature?

\section{SÉPULTURE SP5022}

Mode d'inhumation : indéterminé.

Squelette non conservé, sauf les diaphyses fémorales dont l'extrémité distale est orientée à l'est.

\section{SÉPULTURE SP5023}

Mode d'inhumation: indéterminé. Maturité indéterminée, sexe archéologique: féminin. Tête à l'ouest. Attitude des avant-bras indéterminée.

Mobilier céramique (pl. VI, $\mathrm{n}^{\text {os }} 1$ et 2) : 1 cruche ou gobelet à revêtement argileux $\left(\mathrm{n}^{\circ} 1\right)$, placé latéralement au genou droit ; 1 plat en sigillée claire B $102\left(n^{\circ} 2\right)$, situé latéralement au pied droit.

Mobilier métallique (pl. VI, $\mathrm{n}^{\text {os }} 16-19$ ) (étude de
Michel Feugère) : 3 fragments d'un bracelet rubané en bronze $\left(\mathrm{n}^{\circ} 16\right)$, longueur inconnue, largeur $4,5 \mathrm{~mm}$. Décor composé au centre de deux filets longitudinaux parallèles et sur les côtés d'encoches en " $V$ » régulièrement disposées. Ce bracelet se rattache aux séries rubanées fréquemment attestées aux $\mathrm{II}^{\mathrm{e}}$ et $\mathrm{V}^{\mathrm{e}} \mathrm{s}$. de notre ère. 1 bracelet rubané en bronze $\left(n^{\circ} 17\right)$, diamètre $70 \mathrm{~mm}$, ruban mince de largeur pratiquement constante (4 à $5 \mathrm{~mm}$ ); ce bracelet était fermé par un rivet ; il porte sur toute sa longueur un décor de chevrons estampés, imbriqués les uns dans les autres. Les bracelets rubanés, généralement pourvus d'une fermeture à crochet, se rencontrent sous diverses variantes dans les ensembles du $\mathrm{IV}^{\mathrm{e}}$ et $\mathrm{du} \mathrm{V}^{\mathrm{e}} \mathrm{s}$. Le modèle attesté ici à fermeture fixe (rivetée) n'est pas le plus courant. 1 bracelet en bronze. $\left(\mathrm{n}^{\circ} 18\right)$, diamètre $64 \mathrm{~mm}$, largeur $3,2 \mathrm{~mm}$; une extrémité est brisée, l'autre semble avoir été aménagée en biseau pour une fermeture peut-être soudée à la brasure. Le décor consiste en une succession régulière de plages lisses et bombées et de parties planes ornées d'incisions transversales. 1 bracelet rubané en bronze $\left(n^{\circ} 19\right)$, diamètre inconnu, largeur 4 à $5 \mathrm{~mm}$, bracelet identique au $n^{\circ} 17.1$ bracelet filiforme en deux fragments $\left(n^{\circ} 20\right.$, non dessiné), ornés par endroit de fines incisions transversales. Ces bracelets étaient regroupés en avant du tiers proximal de la diaphyse fémorale droite.

\section{SÉPULTURE SP5038}

Mode d'inhumation : contenant de forme indéterminée, en bois non cloué.

Squelette insuffisamment conservé. Tête à l'ouest. Attitude des avant-bras indéterminée.

Mobilier céramique (pl. VII, $\mathrm{n}^{\text {os }} 1$ et 2 ) : 1 cruche claire B $89\left(n^{\circ} 1\right) ; 1$ coupe non identifiée en céramique commune $\left(n^{\circ} 2\right)$.

\section{SÉPULTURE SP5039}

Mode d'inhumation : indéterminé.

Squelette insuffisamment conservé. Tête à l'est. Attitude des avant-bras indéterminée.

\section{SÉPULTURE SP5041}

Mode d'inhumation : cercueil de forme indéterminée, en bois cloué. 
Squelette insuffisamment conservé. Tête au sud-ouest. Avant-bras ramenés en avant de l'abdomen.

\section{SÉPULTURE SP5042}

Mode d'inhumation : indéterminć.

Squelette insuffisamment conservé. Tête à l'ouest. Avantbras ramenés en avant de l'abdomen.

\section{SÉPULTURE SP5043}

Mode d'inhumation : indéterminé.

Squelette non conservé sauf l'écaille occipitale à l'ouest. Attitude des avant-bras indéterminée.

\section{SÉPULTURE SP5058}

Mode d'inhumation : indéterminé.

Squelette non conservé, sauf fragments du bloc craniofacial à l'ouest.

Mobilier céramique : 1 gobelet non identifié à revêtement argileux (non dessiné).

\section{SÉPULTURE SP5062}

Mode d'inhumation : indéterminé.

Squelette non conservé.

\section{SÉPULTURE SP5067}

Mode d'inhumation : indéterminé.

Squelette non conservé. Petitc fosse (longueur $0,84 \mathrm{~m}$ ) : immature?

\section{SÉPULTURE SP5068}

Mode d'inhumation : dépôt en pleine terre.

Canidé adulte. Placé sur le côté gauche, membres fléchis sauf l'antérieur gauche, tête ramenée sur l'échine, tête au sud.

\section{SÉPULTURE SP5069}

Mode d'inhumation : dépôt en pleine terre.

Canidé adulte. Placé sur le côté droit, membres postérieurs croisés en avant des membres antérieurs, tête au nord. 

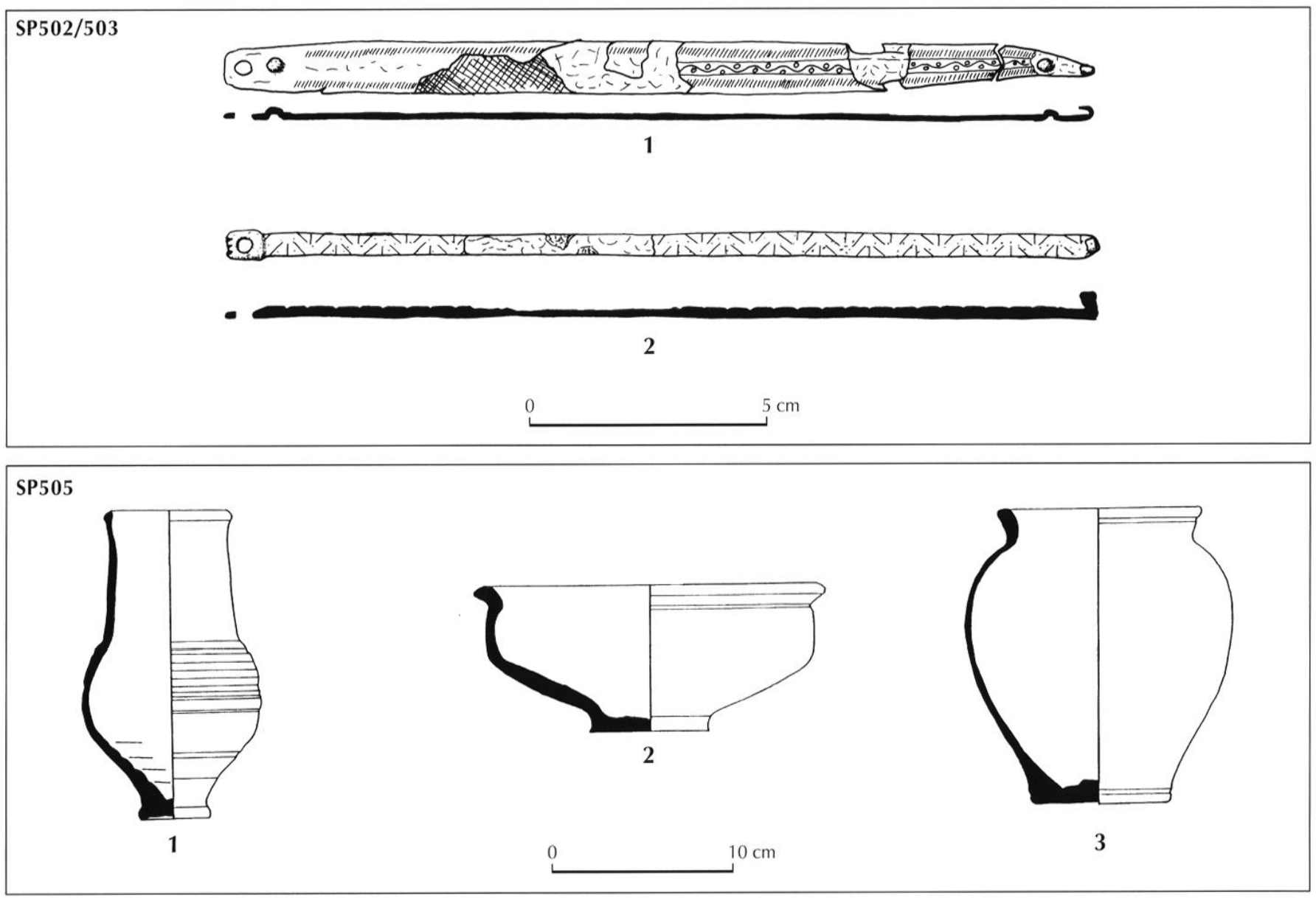

SP506
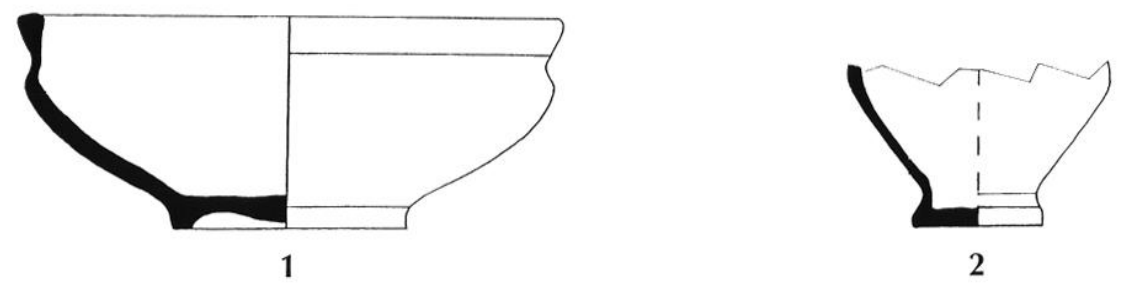

0

$10 \mathrm{~cm}$

SP508

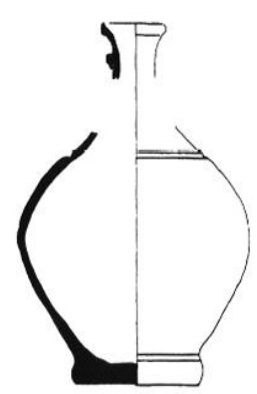

1

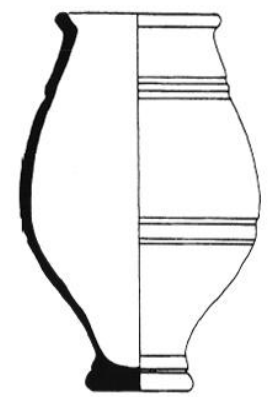

2

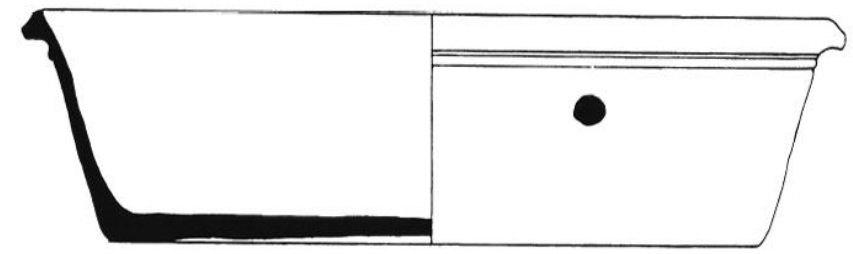

3

$10 \mathrm{~cm}$

Pl. I - Mobilier céramique et métallique trouvé dans les sépultures du Pillon à Marennes.

Sépulture SP502/503:1, 2, bracelets en alliage cuivreux.

Sépulture SP505:1, revêtement argileux ; 2, céramique commune rouge ; 3, céramique commune grise.

Sépulture SP506:1, 2, revêtement argileux. Sépulture SP508:1, 2, revêtement argileux ; 3, céramique commune grise.

(Dessin mobilier céramique : C. Bonnet; mobilier métallique : M. Rolland ; DAO : (.. Macabéo). 


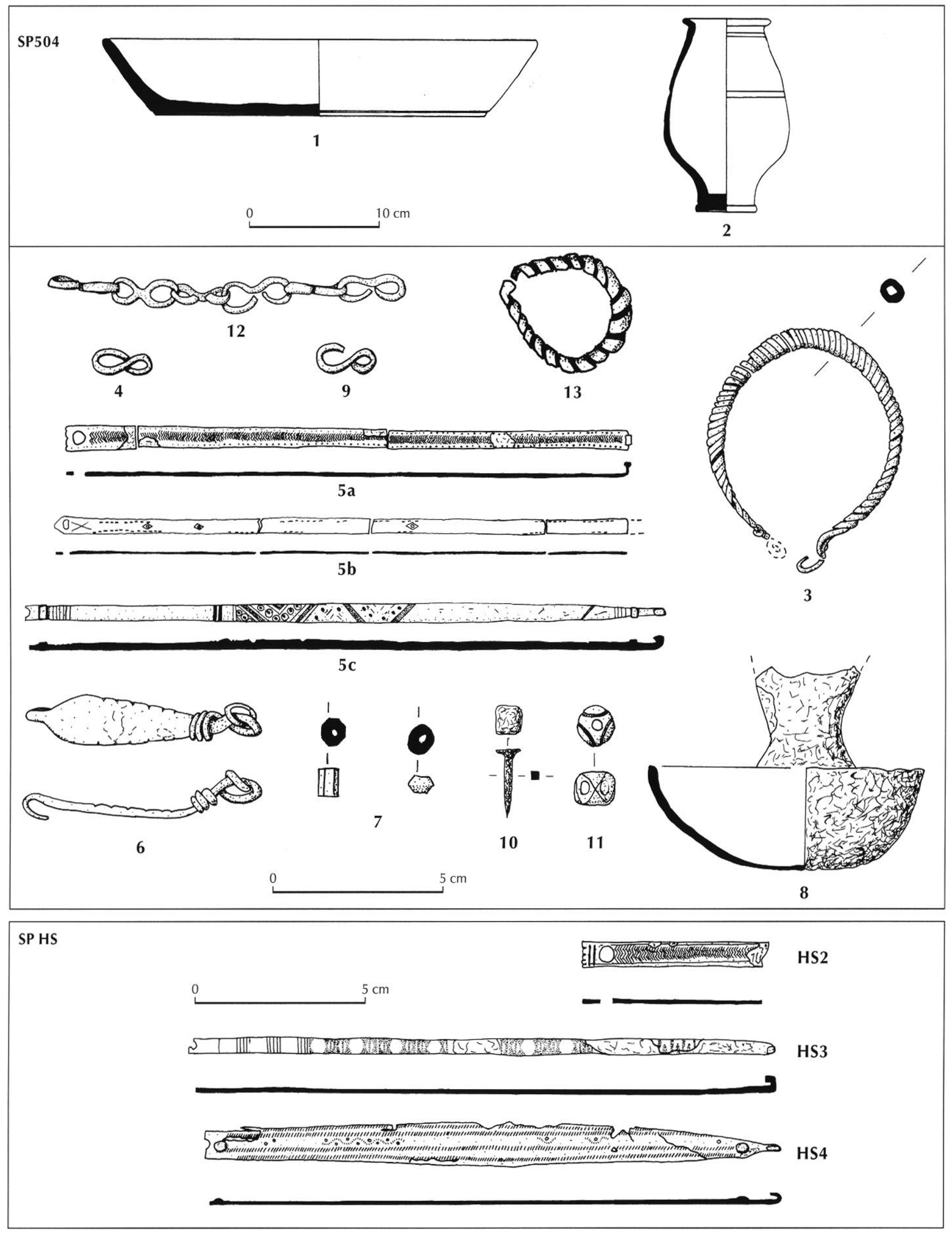

PI. II - Mobilier céramique, métallique et en verre trouvé dans les sépultures du Pillon à Marennes.

Sépulture SP501: 1, 2, revêtement argileux ; 3, 5a-c, bracelets en alliagé cuivreux ; 4, 9, maillons en alliage cuivreux; 6, plaque de ceinture (?) ; 7,11 , perles de verre; 8 , lampe en fer; 10, clou de coffret en fer; 12, chainette en alliage cuivreux; 13, boucle d'oreille en alliage cuivreux. Sépulture HS: HS2, HS3, HS4, bracelets en alliage cuivreux.

(Dessin mobilier céramique: C. Bonnet ; mobilier métallique : M. Rolland; DAO : G. Macabéo). 

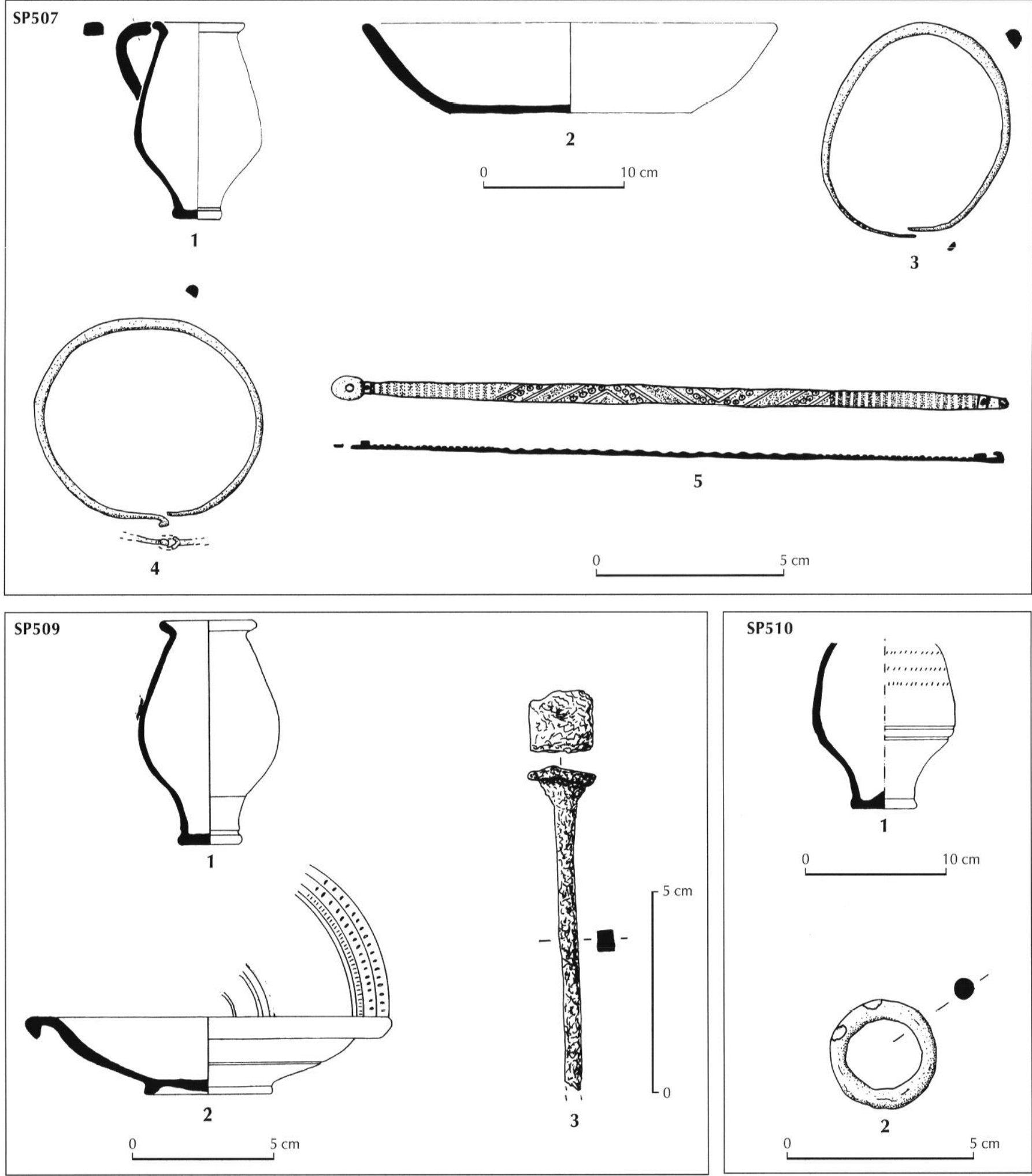

P1. III - Mobilier céramique et métallique trouvé dans les sépultures du Pillon à Marennes.

Sépulture SP507:1, 2, revêtement argileux ; 3-5, bracelets en alliage cuivreux.

Sépulture SP509: 1, 2, revêtement argileux ; 3, clou de cercueil.

Sépulture SP510:1, revêtement argileux; 2 , anneau en alliage cuivreux.

(Dessin mobilier céramique : C. Bonnet; mobilier métallique : M. Rolland; DAO: G. Macabéo). 


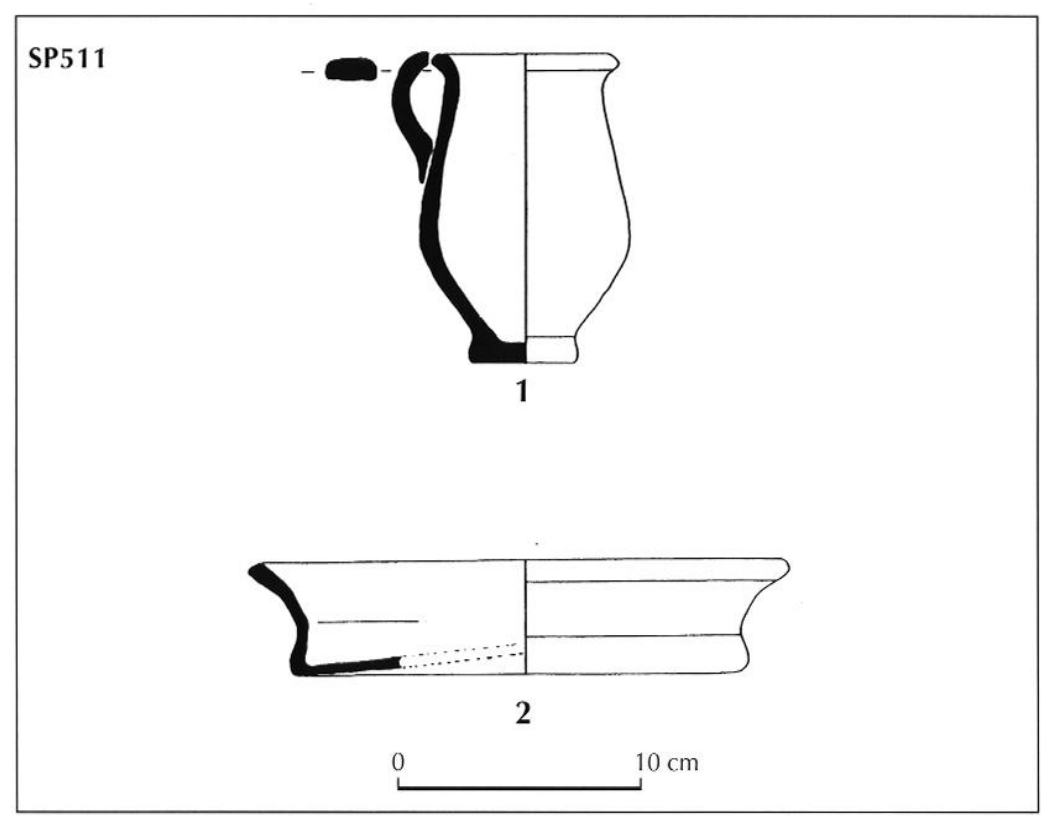

SP513

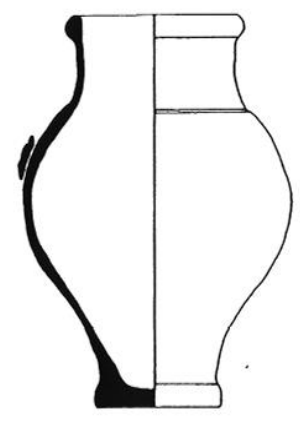

1

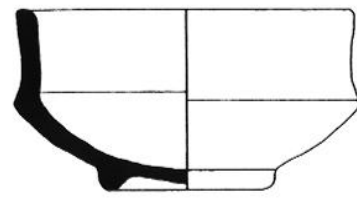

0 $10 \mathrm{~cm}$
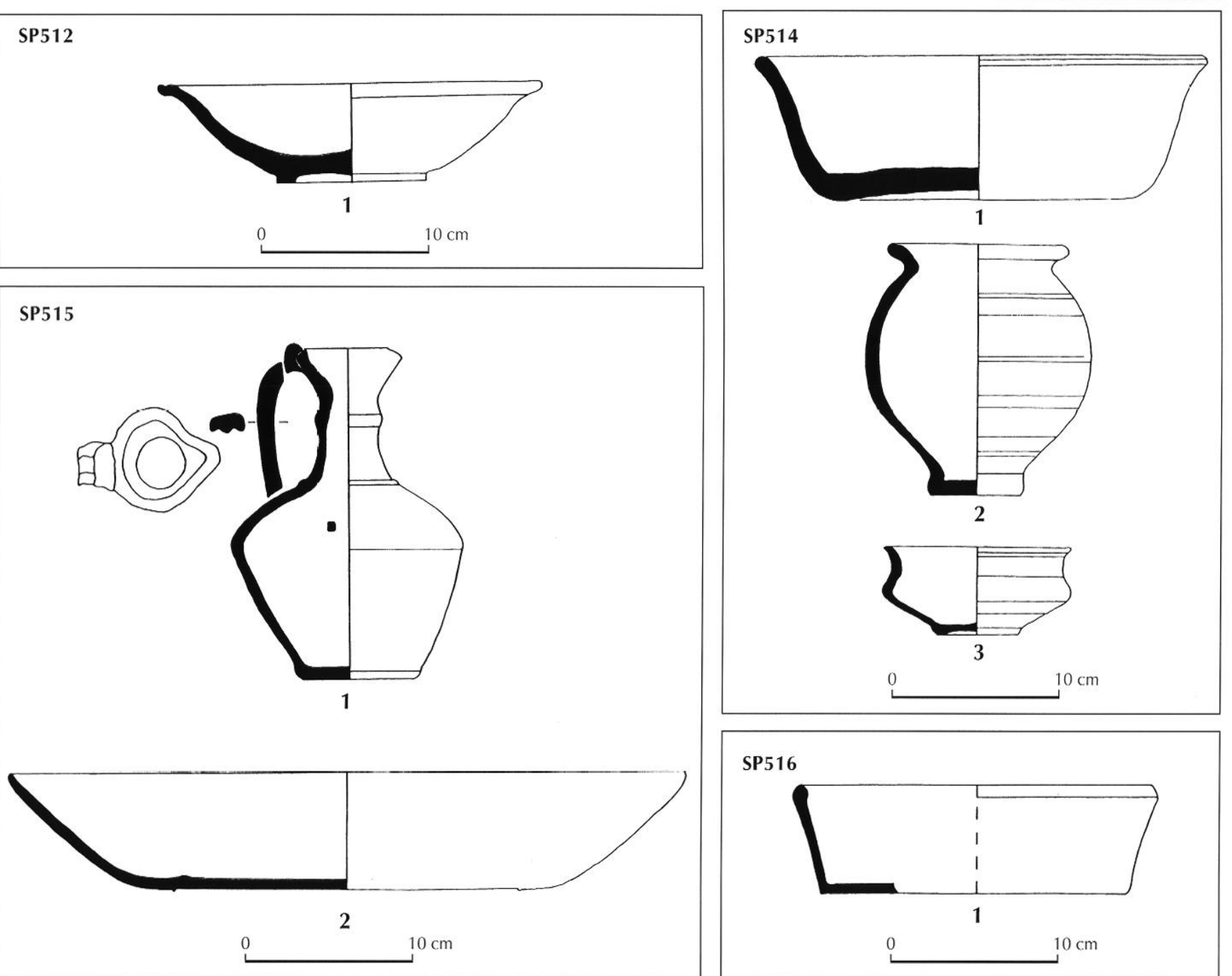

SP516
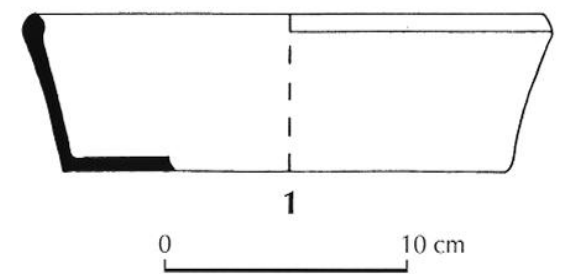

PI. IV - Mobilier céramique trouvé dans les sépultures du Pillon à Marennes. Sépullure SP511: 1, revêtement argileux; 2, céramique commune non identifiée. Sépullure SP512:1, revêtement argileux.

Sépullure SP513:1, 2, revêtement argileux.

Sépulture SP514:1, 2, céramique commune grise; 3, revêtement argileux.

Sépullure $S P 515: 1$, céramique commune rouge ; 2 , sigillée africaine $C$.

Sépulture SP516:1, céramique commune rouge.

(Dessin : C. Bonnel; DAO : G. Macabéo). 

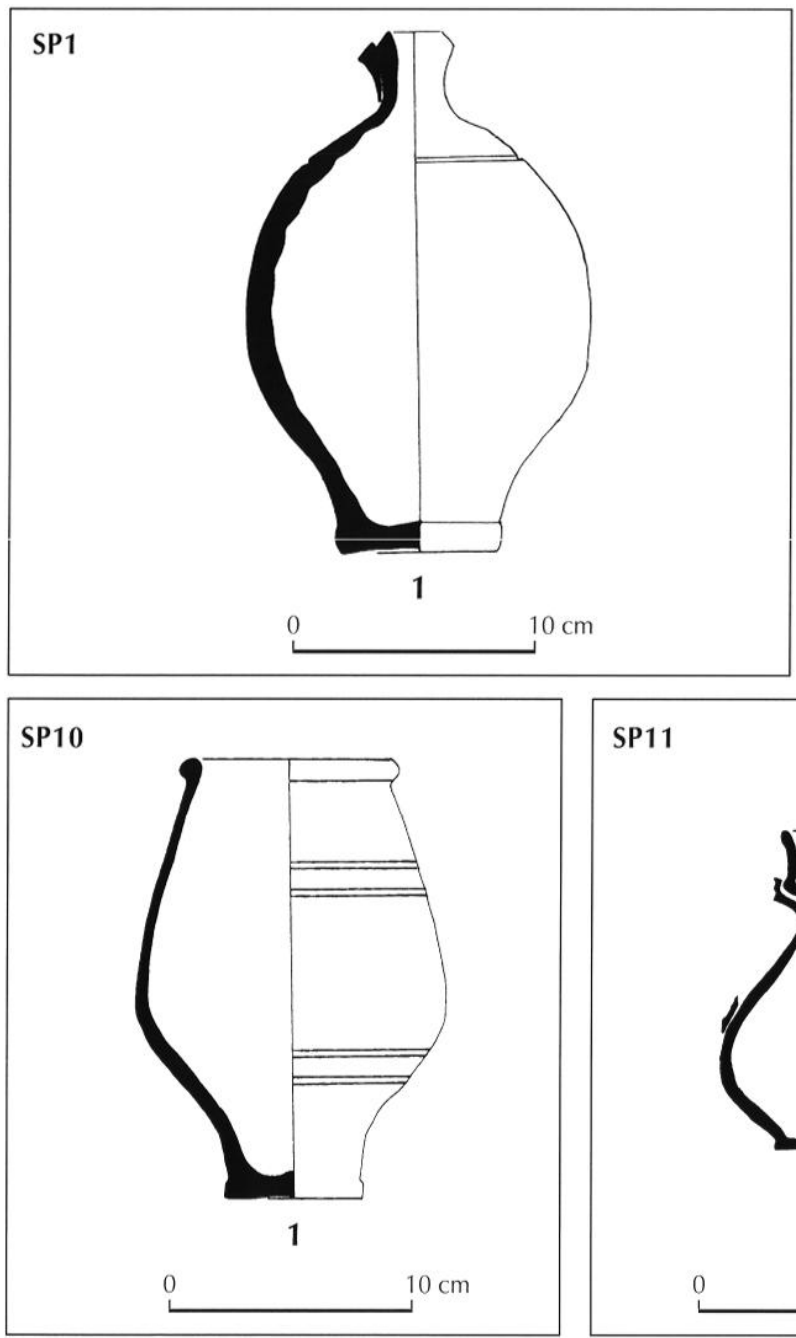

SP11
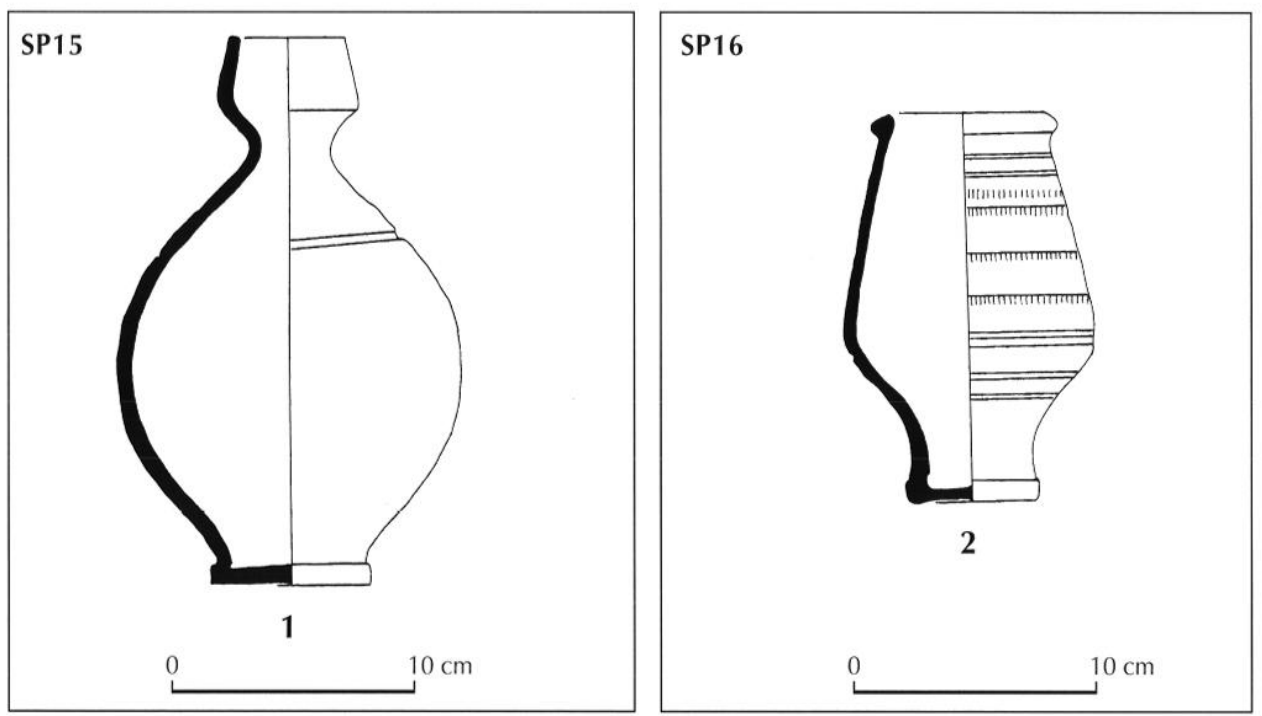

SP2

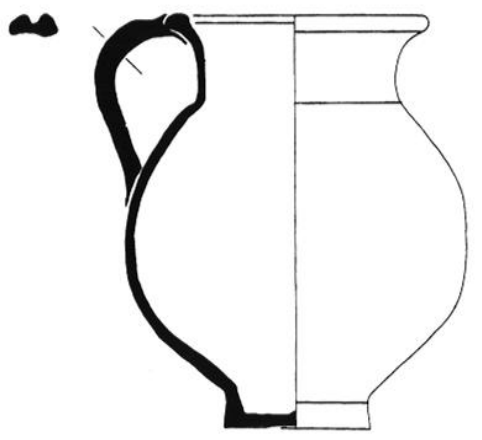

1

0

$10 \mathrm{~cm}$

5 ex.

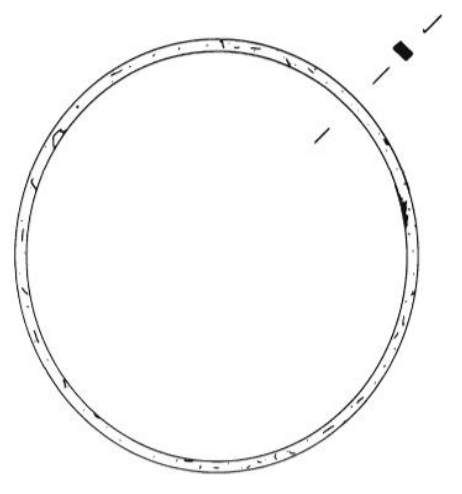

nos 2 à 6

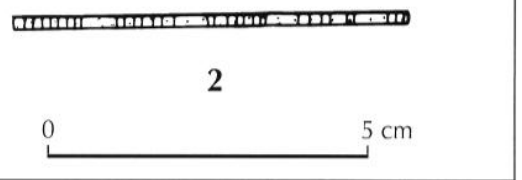

SP27

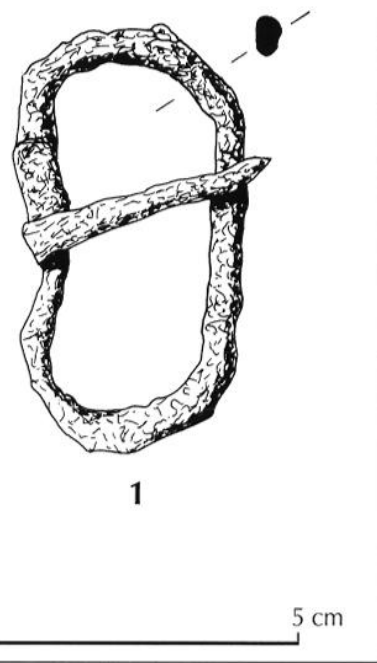

Pl. V - Mobilier céramique et mélallique trouvé dans les sépullures du Trillet à Meyzieu.

Sépulture SPI : 1, revêtement argileux.

Sépulture SP2: 1, céramique commune grise ; 2 (5 exemplaires $n^{\text {as }} 2-6$ ), bracelets en alliage cuivreux.

Sépullure SP10: 1 , revêtlement argileux.

Sépulture $S P 11: 1$, revêtement argileux.

Sépulture SP15: 1 , sigillée claire B.

Sépulture SP16: 2, revêtement argileux.

Sépulture SP27: 1, boucle en fer.

(Dessin mobilier céramique : C. Bonnet et D. Frascone; mobilier mélallique : M. Rolland; DAO : (r. Macabéo). 

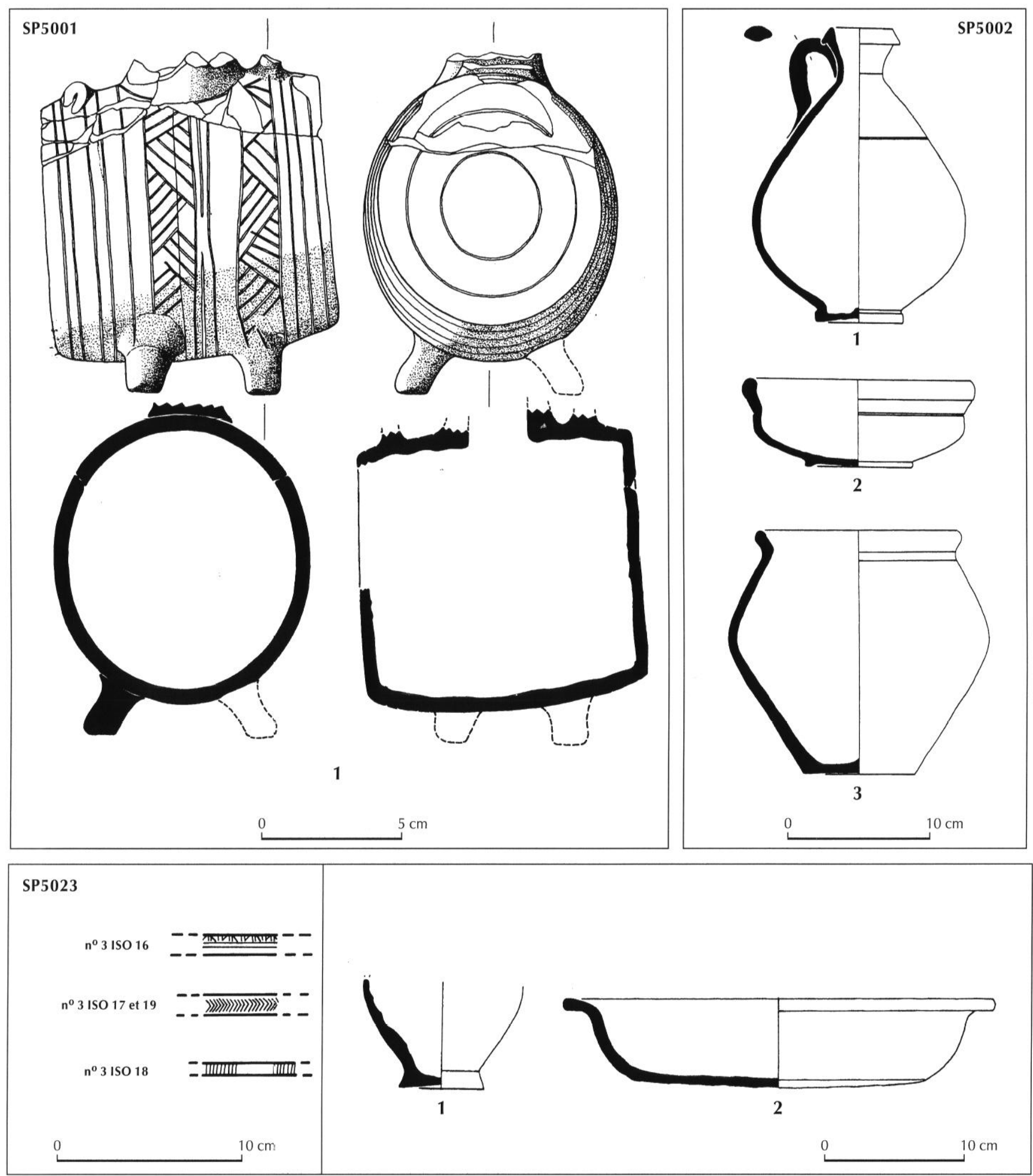

PI. VI - Mobilier céramique et métallique trouvé dans les sépultures des Girardes à Lapalud. Sépulture SP5001: 1 , tonnelet en céramique.

Sépulture SP5002 : 1, sigillée claire B ; 2, céramique commune rouge ; 3, céramique grise kaolinitique.

Sépulture SP5023 : registres décoratifs des bracelets ; 1, 2, revêtement argileux.

(Dessin mobilier céramique: C. Plantevin (SP5001) et C. Bonnet (SP5002 et SP5023); mobilier métallique: M. Rolland; DAO:G. Macabéo). 

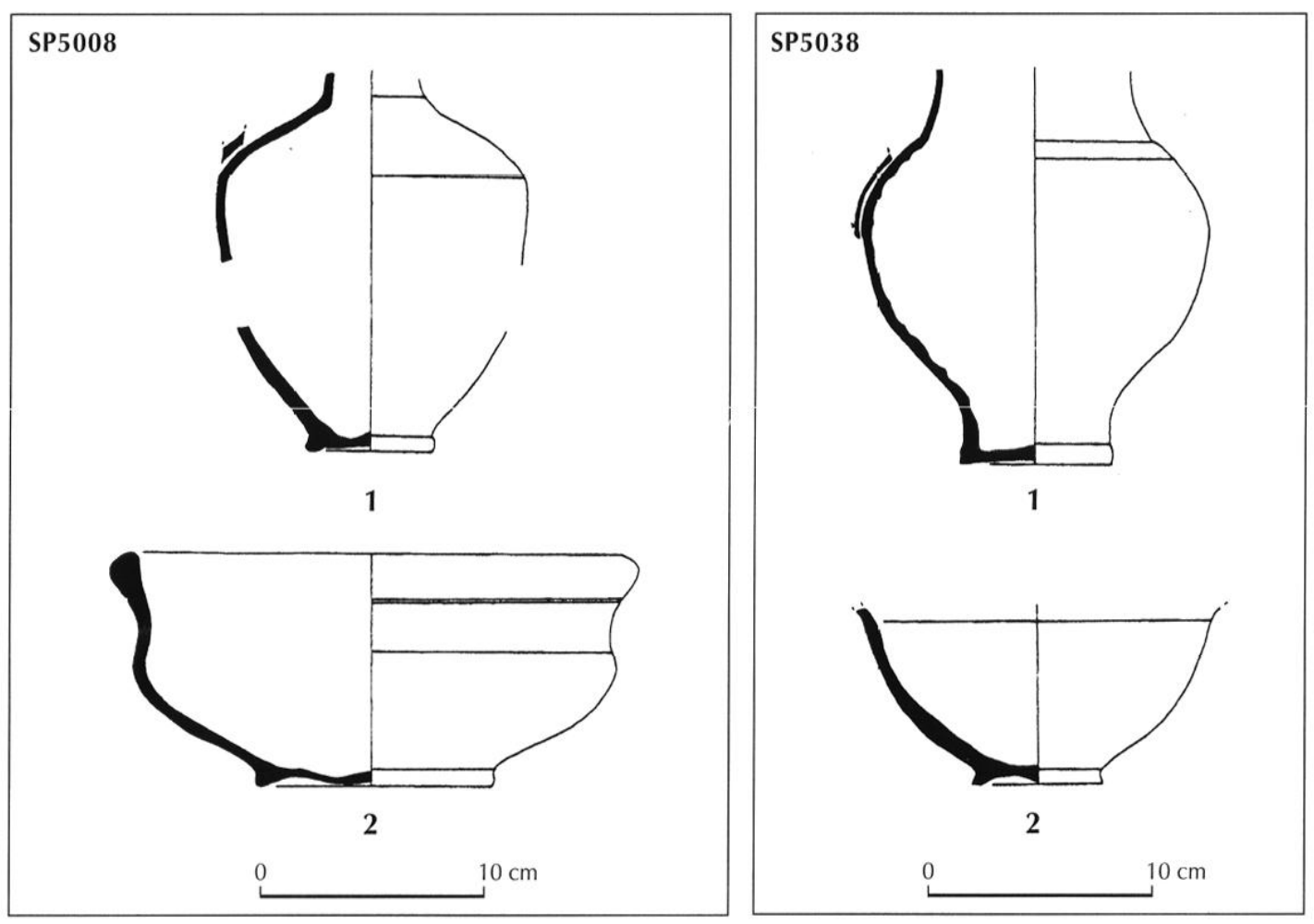

PI. VII - Mobilier céramique trouvé dans les sépultures des Girardes à Lapalud. Sépulture SP5008:1, céramique non identifiée ; 2, céramique commune rouge. Sépulture SP5038: 1, revêtement argileux ; 2, céramique non identifiée.

(Dessins : C. Bonnet ; DAO : G. Macabéo) 


\section{BIBLIOGRAPHIE}

Al. A.MO J.-C.

1986 : La dénomination des productions de vaisselle commune, Reoue archéologique Sites, hors série, 29, 226 p.

Al.IHANGA M.

1979 : Rites de la mort au Gabon, in: Ies hommes et la mort: rituels funéraires à travers le monde, Objets et Mondes, I a Revue du musée de l'Homme, $\mathrm{n}^{\circ}$ spécial, p. 275-280.

Al.ıx G., Blinzot F.

2000 : Nécropole d'immatures de la villa dc Champ-Madame, in: Alfonso G., Alix G., Blaizot F., Cabezuelo U., Lalaï D., Wittmann A. (éds), Beaumonl (Puy-de-Dôme). Bassin d'orage amont de Pourliat, bâtiments annexes et nécropole d'immatures de la villa antique de Champ Madame, Document final de synthèsc, Scrvice régional de l'archćologic d'Auvergne, ClermontFerrand, p. 60-115.

Allain J., Fauduet I., Turrkenu-Libre M. 1992 : l.a nécropole gallo-romaine $d u$ "Champ de l'Image " à Argentomagus (Saint-Marcel, Indre), mémoire I du musée d'Argentomagus, SaintMarcel, 237 p.

AMINT II.

1976: Chronologische Untersuchungen an fränkischen Gräberfeldern der jüngeren Mcrowingerzeit im Rheinland, Berichl der RömischGermanischen Kommission, 57, p. $285-336$.

Arcilin P., Buchinschut\% O.

1985 : Les données de la Protohistoire, in: I asfargues J. (dir), Archileciures de terre el de bois, l'habitat privé des provinces occidenlales du monde romain. Antécédents el prolongements: Protohistoire, Moyen Âge el quelques expériences contemporaines, Actes du $2^{c}$ congrès archéologique de Gaule méridionale, Iyon, 2-6 nov. 1983 ,
Documents d'archéologie française, 2, p. $15-28$.

AUdra A.

1997 : Trouvaille de six folles d'époque constantinienne dans une tombe à Marcnnes (Rhône), Bulletin de la Société française de numismatique, févr. $1997,2,15 \mathrm{p}$.

NYALA G.

2000 : Lyon-Saint-Jean : évolution d'un mobilier céramique au cours de l'Antiquité tardive, Rerue archéologique de l'ksl, 49, p. 207-247.

Ayala G., Cécallon C., Franc O.

2000 : Saint-Priest (Rhône), parc technologique de la Porte des Alpes, mail central: rue Minerve, Document final de synthèse, Service régional de l'archéologic de Rhône-Alpes, Lyon, $51 \mathrm{p}$.

BArThÉlÉmy A., Depierri G.

1990 : La nécropole gallo romaine des Cordiers à Mâcon, Mâcon, ville de Mâcon el ministère de la Culture, $126 \mathrm{p}$.

BASTIEN P.

1980 : Le monnayage de l'alelier de Lyon - De la réforme monétaire de Dioclétien à la fermeture temporaire de l'atelier en 316 (294-316), Wetteren, éd. Numismatique romaine, $274 \mathrm{p}$.

1985 : I e monnayage de l'alelier de Lyon - De la mort de. Conslantin à la mort de Julien (337-363), Wetteren, éd. Numismatique romaine, $263 \mathrm{p}$.

Batigne C., Desbat A.

1996 : Un type particulicr de cruche : les bouilloires en céramique d'époque romaine (I ${ }^{\mathrm{cr}}$-III $\mathrm{I}^{\mathrm{c}}$ siècles), in: Actes $d u$ congrès de la SFECAC, Dijon, 1996, Marseille, SFECAG, p. 381-394.

BE:L. V.

1992 : Recherches sur la nécropole galloromaine du Valladas à Saint-Paul-Trois-
Châleaux, Thèse dactylographiée, Aix-en-Provence, 1084 p.

1996 : Étude spatiale de sept incinérations primaires gallo-romaines de la région lyonnaise, in: Méthodes d'études des sépultures: du terrain à l'interprélation des ensembles funéraires, Actes du colloque du GDR 742 du CNRS 1995 , Bulletins et mémoires de la Société d'anthropologic de Paris, 8, 3-4, p. $207-222$.

\section{Bei, V., Mannie7. Y.}

1996 : Pcrmanences et mutations des pratiques funéraires au III $\mathrm{s}$. dans le sud-est de la Gaulc, in: Fiches J.-L. (dir.), Le III's. en Caule Narbonnaise : données régionales sur la crise de l'Empire, Actes de la table ronde du GDR 954 du CNRS, Aix-en-Provence, La Baume, 15-16 sept. 1995, Sophia Antipolis, éd. APDCA, p. 83-102.

Bri. V., Tranoy L., Béraud J., Gébara C. 1991 : Les nécropoles à incinération et à inhumation en Gaule méridionalc, in: Incinérations et inhumations dans l'Occident romain aux trois premiers siècles de notre ère, Actes du colloque de Toulousc-Montréjeau, IVc congrès archćologique de Gaule méridionale, 7-10 oct. 1987 , p. $9-40$.

Belllamy P. S., Hitchner R. B., MCKINLEY J. I.

1996 : The villas of the Vallée des Baux and the Barbcgal Mill : excavations at La Mérindole villa and cemetery, Journal of Roman Archaeology, 9, p. 154-186.

BENNCER A.

1992 : Meyzieu-Decines Le Trillet 1992 : nécropole, étude anthropologique, Rapport préliminaire, Scrvicc régional de l'archéologie de RhôneAlpes, Lyon, 250 p.

BÉRARD G.

1963 : La nécropole gallo-romaine de La Calade à Cabasse (Var), deuxième 
campagne de fouilles (1962), Gallia, XXI, 2, p. 295-306.

Beir P., Frente A., Montint:Ri D.

1989 : La typologie de la sigillée lisse de Lezoux : considérations générales et formes inédites, in : Actes du congrès de. la SFICAC; Lezoux, 1989, Marseille, SFECAG, p. 37-54.

BNTLIF J., HAMROW H.

1995 : Europe between Lale Antiquity and the Middle. Ages, British Archaeological Report, International Series, 617, $117 \mathrm{p}$.

BLAI\%OT F.

1997 : L'ensemble funéraire, phase 17Dl, in: Pomarèdes H. (éd.), La Ramière-Roc de Peillet (Roquemaure, Gard), l'espace graire et les établissements ruraux gallo-romains, l'établissement moderne du Roc de Peillet, Document final de synthèse, opération TGVMéditerranée, Service régional de l'archéologie du LanguedocRoussillon, Montpellier, p. 118-143.

1998a: L'ensemble funéraire, in: Flotté P., Blaizot F. (éds), Cilinique Sainle-Barbe, Sirasbourg, Document final de synthèse, Service régional de l'archéologie d'Alsace, Strasbourg, p. 39-104.

1998b : La reconnaissance des dispositifs en matière périssable et leur interprétation. Exemples tardo-antique dans la Drôme et alto-médiéval en Seine-et-Marne, in: Rencontre autour du cercueil, Compte rendu de la journée d'étude organisée par le Groupe d'archéologie et d'anthropologie funéraire d'île-de-France, Paris, 28 janv. 1997, Publication du Service départemental d'archéologie du Vald'Oise, Paris, Association pour les fouilles archéologiques nationales, p. 79-84.

À paraître: L'ensemble funéraire "Le Patis » à Montboucher-sur-Jabron (Drôme), Mémoires d'archéologie méridionale.

BiAizoT F., AYAla G.

1996 : Montboucher-sur-Jabron, Le Patis I, nécropole, Document final de synthèse, Service régional de l'archéologie de Rhône-Alpes, Lyon, $130 \mathrm{p}$.

\section{B1.AI\%) F., SiVINO V.}

À paraître: Sépultures et ensembles funéraires dispersés du haut Moyen Âge dans la moyenne valléc du Rhône, in: Maufras O. (éd.), Habilats, sépultures, paysage el économie. aii Moyen Age dans io sud-esi de in France, Documents d'archéologic française.

Blasco C., Fliugìre M., Jlannot D., RiYNALD C.

1987 : Nécropoles de Quarante (Hérault), in: Raynaud C. (dir.), Nécropoles languedociennes de l'Antiquilé tardive el du haul Moyen Âge, Archéologie en Languedoc, 4, p. 133-142.

\section{BOIRON R.}

1993 : Les nécropoles de Clavelles et de Saint-Martin (Alpes-de-HauteProvence), I"'-VII"s. de notre ère, in: Ferdière A. (dir.), Monde des morts, monde des vivants en Gaule rurale, Actes du colloque ARCHEA/AGER, Orléans, 7-9 févr. 1992, 6 $6^{\circ}$ suppl. à la Revue archéologique du Centre de la France, p. 325-332.

Bolssinot P., Rogik K., Bl.Al\%oT F.

1998 : Lapalud Les Girardes. Vaucluse (84), TGV ligne 5-Secteur II : Valence-Avignon, Document final de synthèse, Service régional de l'archéologie de Provence-Alpes-Côte-d'Azur, Aixen-Provence, non paginć.

\section{BONNET C.}

À paraître: Contribution à l'étude des céramiques culinaires de la fin du II"s. au milieu du V“s. apr. J.-C. de la plaine valentinoise au nord du Tricastin, d'après le mobilier issu des fouilles du TGV-Méditerranée, Revue archéologique de Narbonnaise.

\section{BORZA H.}

1955: Le mythe de l'obole à Charon et le symbolisme actuel de la monnaie dans le cercueil, ORBIS, Bulletin inter- national de documentation linguistique, IV, 1, Louvain, p. 1.34-I48.

BOURGeis I.

1986 : Les nécropoles gallo-romaines en Île-de-France: un état de la recherche, in: Gallo-Romains on Îlode-Irunce, Paris, Association des conservateurs des musées d'île-deFrance, p. 290-312.

BOYT:R R.

1987 : Vie et mort à Marseille à la fin de l'Antiquité, Marseille, éd. Atelier du Patrimoine, $123 \mathrm{p}$.

BRÉNOT C.

1986 : La circulation monétaire sur les sites de Lyon à la Méditerranée (IV"VII" siècles), in: Premiers temps chrétiens en Gaule méridionale (Antiquité tardive et haut Moyen Âge, $I I T$ VIII' siècles), Catalogue d'exposition, Lyon, 1986, p. 197-198.

Brogua C.

1998 : Approche bayesienne et décision : application à une méthode d'estimation de l'âge au décès (Iovejoy et al. 1985), mémoire de DEA, univ. Bordeaux 1, 100 p.

BRULET R.

1990 : Ia Gaule septentrionale du BasEmpire. Occupation du sol et défense du territoire dans l'arrière-pays du limes aux $I V^{*}$ el $V^{\prime} s$., Trierer Zeitschrift für Geschichte und Kunst des Trierer Landes und Seiner Nachbargebiete, Beiheft 11, Trier.

1993 : L'archéologie funéraire et l'occupation des sols au Bas-Empire en Gaule du Nord, in: Struck M. (ed.), Römerzeilliche (iräber als Quellen zu Religion, Bevölkerungsstruklur und Sozialgeschichte, Actes du colloque de Mayence, 18-20 févr. 1991, Archäologische Schriften des Instituts für Vor- und Frühgeschichte der Johannes Gutenberg-Universität, Mainz, 3, p. 403-412.

BRUZEK J.

1991 : Fiabilité des procédés de détermination du sexe à partir de l'os coxal. Implications à l'étude du dimorphisme sexuel de 
l'Homme fossile, thèse de Doctorat, Paris, Muséum national d'histoire naturelle, Institut de paléontologie humaine, 431 p. et 102 p. d'annexes.

Bruzek J., Castex D., Majo T.

1996 : Évaluation des caractères morphologiques de la face sacro-pelvienne de l'os coxal : approche pour une proposition d'une méthode de diagnose sexuelle, in: Méthodes d'études des sépultures: du terrain à linterprétation des ensembles funéraires, Actes du colloque du GDR 742 du CNRS 1995, Bulletins et mémoires de la Société d'anthropologie de Paris, 8, 3-4, p. 491-502.

Carku D., Boccacino C., Borgard P., Boulllot J., Buchet L., BuIssonCatil.J., Vattéoni S.

1991 : Une nécropole de l'Antiquilé tardive à Vaison-la-Romaine: les fouilles des quartiers du Colombier et de Pommerol, Documents d'archéologie vauclusienne, 2, $97 \mathrm{p}$.

\section{Castex D.}

1994 : Morlalité, morbidité et gestion de l'espace funéraire au cours du haul Moyen Âge. Contribution spécifique de l'anthropologie biologique, thèse de Doctorat, univ. Bordeaux I, spécialité anthropologie, n 1097, 2 vol., 500 p., inédit.

\section{Castex D., Friess M.}

À paraître: Ein Pestsommer in der Provence-Demographische Anomalien im Kontext von Epidemien, in: Henke W., Kemkes-Grottenthaler A. (eds), Epidemiologie, Actes du colloque de Francfort, 1998.

CASTOLdI M., FEugÈRE M.

1991 : Les Simpulums, in: La vaisselle. tardo-républicaine en bronze, Centre de recherches sur les techniques gréco-romaines, Dijon, Univ. de Bourgogne, p. 61-88.

\section{CÉCILIon C.}

1999 : Les monnaies de la nécropole à incinération du site de la villa Montel à Lyon 9 , in: Le Nézet M. (éd.), Rapport provisoire (présentation des données de terrain) du site de la villa Montel, rue du Bourbonnais (Lyon 9c) déposé au Service régional de l'archéulugie de Rhône-Alpes, Lyon.

2001 : Petits bronzes de la dynastie constantinienne découverts à SaintPriest (Rhône), au lieu-dit "Minerve ", Bulletin de la Société française de numismatique, $56^{\mathrm{c}}$ année, $\mathrm{n}^{\circ} 2$, p. 21-27.

Cinastel J., Plassot E., Thiériot F., 1995 : Le quartier Saint-Pierre ; la voie et les sépultures gallo-romaines, in: Delaval E., Bellon C., Chastel J., Plassot E., Tranoy L. (éds), Vaise, un quartier de Lyon antique, Documents d'archéologie en Rhône-Alpes, 11, p. $36-70$.

\section{Châtelet M.}

1998 : L'évolution de la céramique culinaire tournée, du $\mathrm{V}^{\mathrm{c}}$ au $\mathrm{X}^{\mathrm{e}}$ siècle, dans le sud du Rhin supérieur (Alsace et Pays de Bade), in: Delestre X., Périn P. (éds), La datation des structures et des objets $d u$ haut Moyen Âge: mélhodes et résultats, Mémoires publiés par l'Association française d'archéologie mérovingienne, VII, p. 21-38.

ChOUQuer G., FAVORY F.

1980 : Contribution à la recherche des cadastres antiques, Paris, Centre de recherches d'histoire ancienne, 31, $90 \mathrm{p}$.

\section{COLARDELIL. M.}

1983 : Sépullures et traditions funéraires $d u$ $V^{p}$ au VIII siècle ap. J.-C. dans les campagnes des Alpes françaises du Nord, Grenoble, Socićtć alpinc de documentation et de recherche en archéologie historique, $464 \mathrm{p}$.

Colarideli.e M., Demians d'Archimbaud G., Raynaud C.

1996 : Typo-chronologie des sépultures du Bas-Empire à la fin du Moyen Âge dans le sud-est de la Gaule, in: Galinié H., Zadora-Rio E. (éds), Archéologie du cimetière chrétien, Actes du $2^{c}$ colloque ARCHEA, Orléans, 29 sept.- $^{\text {er }}$ oct. 1994, Tours, $11^{\mathrm{e}}$ suppl. à la Revue archéologique du Centre de la France, p. 271-303.

\section{Collectif}

1987 : Nécropoles à incinération du HautEmpire, Actes de la table ronde de Lyon, 30-31 mai 1986, Rapports archéologiques préliminaires de la région Rhône-Alpes, Lyon, $142 \mathrm{p}$.

1998 : Pré, Proto, Gallo, Méro. Histoire de l'Ain en archéo, Catalogue d'exposition, Musée de Brou, 27 juin-13 déc. 1998, Bourg-en-Bresse, 143 p., $437 \mathrm{fig}$.

\section{Collis J.}

1977 : Owslebury (hants) and the problem of burials on rural settlements, in: Reece R. (ed.), Burial in the Roman World, Research Report, 22 , p. 16-34.

\section{Coquidé C., Vermeulin C.}

1999 : Évolution d'une zone d'habitat rural du $\mathrm{I}^{\mathrm{er}}$ s. av. J.-C. au III ${ }^{\mathrm{e}}$ s. apr. J.-C. : Chassieu-Genas L'épine (Rhône), Revue archéologique de Narbonnaise, 32, p. 197-244.

Cordy J.-M., Udrfacu M., Yernaux G.

1995 : Étude ostéologique des restes d'animaux du vicus gallo-romain à Braives (prov. de Liège, Belgique), in: Homme et animal dans l'Antiquilé romaine, Actes du colloque de Nantes 1991, Tours, Centre de recherches A. Piganiol, p. 131-144.

Crummy N., Crummy P., Crossan C.

1993 : Excavations of Roman and later cemeteries, churches and monastic sites in Colchester, 1971-1988, Colchester Archaeological Report, 9, Norfolk, Witley Press, Hunstanton, $298 \mathrm{p}$.

\section{Darfmbirt C., Saglio E}

1969 : Funus, in: Dictionnaire des Antiquités grecques et romaines d'après les textes et les monuments, Graz, Akademische Druck- u. Verlagsanstalt, p. $1367-1409$.

DEPEYROT $\mathrm{G}$.

1982 : Le numéraire gaulois du $V^{e}$ siècle. 
Aspects quantitatifs, British Archaeological Report, 127, 2 vol., 273 p. et $190 \mathrm{p}$.

1987 : Le Bas-empire romain. Économie et numismatique (284-491), Paris, éd. Errance, 139 p.

DESAYE $\mathrm{H}$.

1996 : Topographie et monuments de Dea Augusta (Die), Revue drômoise, I.XXXX, 482, p. 177.

Desbat A.

1988 : La sigillée claire B : état de la question, in: Actes du congrès de la SFECAG, Orange, 1988, Marseille, SFECAG, p. 91-98.

1991 : Un bouchon de bois du $I^{\mathrm{er}} \mathrm{s}$. après J.-C. recueilli dans la Saône à Lyon et la question du tonneau à l'époque romaine, Gallia, 48, p. 319-336.

1994 : La place du tonneau dans l'économie gallo-romaine sous le HautEmpire, in: Boire et manger en Bourgogne. Usages et fonctions $d u$ mobilier archéologique, du Néolithique au Moyen Âge, Actes des $2^{c}$ journées de rencontres d'ARIA Bourgogne à Auxerre, 7-8 mars 1992, Dijon, DRAC de Bourgogne, p. 87-98.

Desbat A., Picon M.

1986 : Sigillée claire B et " luisante ": classification et provenance, Figlina, 7, Laboratoire de céramologie de Lyon, p. 5-18.

\section{DICOCER}

1993 : Dictionnaire des céramiques antiques (VII s. av. n. è.-VII s. de n. è.j en Méditerranée nord-occidentale (Provence, Languedoc, Ampurdan), Lattara, 6, Lattes, ARALO, 624 p.

Duday H., Laubenheimer F., Tillier A.-M. 1995 : Sallèles-d'Aude, nouveau-nés et nourrissons gallo-romains, Annales littéraires de l'université de Besançon, 563, Paris, Les Belles Lettres, 121 p.

\section{DURAND M.}

1988 : Archéologie du cimetière médiéval au sud-est de l'Oise. Relations avec l'habitat et évolution des rites et des pratiques funéraires $d u V^{e}$ au $X V V^{e}$ siècle, $\mathrm{n}^{\circ}$ spécial à la Revue archéologique de Picardie, $275 \mathrm{p}$.

Favory F., Parodi A., Poupet P., Raynaud C.

1994 : Lunel-Viel et son territoire, in: Favory F., Fiches J.-L. (éds), Les campagnes de la France méditerranéenne dans l'Antiquité et le haut Moyen Âge, études microrégionales, Documents d'archéologie française, 12, p. 163-245.

FERBER E.

À paraître: L'ensemble funéraire "Grange-Neuve " à Allan (Drôme), Mémoires d'archéologie méridionale.

Ferber E., Ronco C.

1996 : Allan "Grange-Neuve " 2, Drôme. Document final de synthèse, opération TGV-Méditerranée, sept. 1996, Service régional de l'Archéologie de Rhône-Alpes, Lyon, $53 \mathrm{p}$.

FERDIÈRE A. (DIR.)

1993 : Monde des morts, monde des vivants en Gaule rurale, Actes du colloque ARCHEA/AGER, Orléans, 7-9 févr. $1992,6^{\text {e }}$ suppl. à la Revue archéologique du Centre de la France, $454 \mathrm{p}$.

Ferembach D., SCHWIDETZKY I., STLOUKAL M.

1979 : Recommandations pour déterminer l'âge et le sexe sur le squelette, Bulletins et mémoires de la Société d'anthropologie de Paris, XIII, 6, p. 7-45.

FERRANDo P.

1997 : Les monnaies d'Arles - De Constantin le Grand à Romulus Avgrustule (313476), Le Poiré-sur-Vie, P. Ferrando, $254 \mathrm{p}$.

FEUGÈre M.

1993 : L'évolution du mobilier non céramique dans les sépultures antiques de Gaule méridionale (II ${ }^{\mathrm{e}} \mathrm{s}$. apr. J.-C.-début du Vc s. apr. J.-C.), in : Struck M. (ed.), Römerzeitliche Gräber als Quellen zu Religion, Bevölkerungsstruktur und Sozialgeschichte,
Actes du colloque de Mayence, 18-20 févr. 1991, Archäologische Schriften des Instituts für Vor- und Frühgeschichte der Johannes Gutenberg-Universität, Mainz, 3, p. 119-165.

Feugère M., Joly J.-P., Pel.lecuer C., PEYRE A.

1987 : La nécropole gallo-romaine tardivc dc La Brèche (Laưdunin, Gard), premières données, in: Raynaud C. (dir.), Nécropoles languedociennes de l'Antiquité tardive et du haut Moyen Âge, Archéologie en Languedoc, 4, p. 81-90.

FINGERLIN G.

1971 : Die alamannischen Gräberfelder von Güttingen und Merdingen in Südbaden. Germanische Denkmaäler der Völkwanderungszeit, série $A, n^{\circ} 12$, Berlin.

FRASCone D., StANIASZEK L.

1992 : Rocade est de Lyon Meysieu-Decine : Le Trillet 1992, habitat et nécropole, Document final de synthèse, Service régional de l'archéologie de RhôneAlpes, Lyon, 94 p.

FRÉville C., JouRna R.

1994 : La nécropole mérovingienne de Longueil-Annel (Oise), Revue archéologique de Picardie, 1/2, p. 87-130.

GAGNIÈRE S.

1965 : Les sépultures à inhumation du III $^{\mathrm{e}}$ au XIII $\mathrm{s}$. de notre ère dans la basse vallée du Rhône, Cahiers rhodaniens, XII, p. 53-110.

GAGniÈRE S., Granier J.

1972 : La nécropole gallo-romaine et barbare de la Font-du-Buis à Saze (Gard), Revue archéologique de Narbonnaise, 5, p. 117-146.

Garnotel A., Raynaud C.

1996 : Groupés ou dispersés ? Les morts et la société rurale en Languedoc oriental (IVe-XII ${ }^{e}$ siècles), in: Galinié H., Zadora-Rio E. (éds), Archéologie du cimetière chrétien, Actes du $2^{\mathrm{e}}$ colloque ARCHEA, Orléans, 
29 sept.- $^{\text {er }}$ oct. 1994, Tours, $11^{\mathrm{e}}$ suppl. à la Revue archéologique du Centre de la France, p. 139-152.

\section{GÉbara C., Pasqualini M.}

1993 : Sépultures et cimetières ruraux en Provence orientale à l'époque gallo-romaine, in: Ferdière A. (dir.), Monde des morts, monde des vivants en Gaule rurale, Actes du colloque ARCHEA/AGER, Orléans, 7-9 févr. 1992, 6 $6^{\text {e }}$ suppl. à la Revuc archéologique du Centre de la France, p. 341-366.

GiNOUVÈs O., SCHNEIDER L.

1987 : Les nécropoles de l'Antiquité tardive et du haut Moyen Âge dans la moyenne vallée de l'Hérault., essai d'inventaire et approche de l'environnement social, in: Raynaud $\mathrm{C}$. (dir.), Nécropoles languedociennes de l'Antiquité tardive el du haut Moyen Âge, Archéologie en Languedoc, 4, p. 91-98.

Girard A., Raynaud C.

1982 : Une nécropole du IV siècle de notre ère à Lansargues (Hérault), Documents d'archéologie méridionale, 5, p. 159-167.

GIRARD L.

1997 : Les sujets immatures du cimetière gallo-romain de Chantambre (Essonne). Pratiques funéraires, in: Buchet L. (dir.), L'enfant, son corps, son histoire, Actes des $7^{\mathrm{e}}$ journées anthropologiques de Valbonne, 1-3 juin 1994, Sophia Antipolis, éd. APDCA, p. 211-225.

GRINSELI. L. V.

1961 : The breaking of oljjects as a funerary rite, Folklore, 72 , p. 475-491.

Hal.bout P., Le Maho J.

1984: Aspects de la construction de bois en Normandie du Irr au XIV siècle. Caen, Centre archéologique de Normandie, $107 \mathrm{p}$.

HaYes J. W.

1972 : Late Roman pottery, a catalog of Roman fine wares, London, The British School at Rome, 477 p.
HOFMANN B.

1959 : La quincaillerie antique, Notices techniques 14,15 et 16 du Touring Club de France, Paris, non paginé.

HunOT J.-Y.

1996 : Les cercueils de bois médiévaux et modernes en Anjou: meubles précieux ou simples caisses ?, Reoue archéologique de l'Ouest, 13, p. 185-203.

IsCAN M. Y. (ED.)

1989 : Age Markers in Human Skeleton, Springfield, Illinois, Charles C. Thomas, $359 \mathrm{p}$.

JOLY D.

1991 : Quatre briques pour un cencueil, in: Compte rendu de la table ronde des 8 , 9 et 10 mai 1991 du GDR 742 du CNRS, Saintes, dactylographié, p. 151-153.

JosPIN J.-P.

1998-1999: Pratiques funéraires des nécropoles antiques d'Aoste (Isère), Evocations, 1998-1999, Grenoble, p. 11-29.

Key C. A., Aiello L. C., Moll.esson T.

1994 : Cranial suture closure and its implication for agc estimation, International Journal of Osleoarchaeology, 4, p. 193-207.

\section{LAMBOGLIA N.}

1963 : Nuove osservazioni sulla « terra sigillata chiara " II, tipi C, lucente et $\mathrm{D}$, Revue d'études ligures, XXIX anncee, 1-4, p. 145-212.

\section{LEDERMANN S.}

1969 : Nouvelles tables types de mortalité, Paris, INED, Travaux et documents, 53, $263 \mathrm{p}$.

LEMOINF. J.

1979 : La mort et ses rites chez les Hmong, in: Les hommes et la morl: rituels funéraires à travers le monde, Objets et Mondes, La Revue du musée de l'Homme, $\mathrm{n}^{\circ}$ spécial, p. 196-207.

LEPETZ S.

1993 : Les restes d'animaux dans les sépultures gallo-romaines, in: Ferdière A. (dir.), Monde des morts, monde des vivants en Gaule rurale, Actes du colloque ARCHEA/AGER, Orléans, 7-9 févr. 1992, $6^{\mathrm{c}}$ suppl. à la Revue archéologique du Centre de la France, p. 37-44.

LORREN C., PÉRIN P.

1995 : L'habitat rural du haut Moyen Âge (France, Pays-Bas, Danemark el CrandeBretagne), Actes des XIV journées internationales d'archéologie mérovingienne, Guiry-en-Vexin et Paris, 48 févr. 1993, Mémoires de l'Association française d'archéologic mérovingiennc, 6, $260 \mathrm{p}$.

\section{Maertins J.-T.}

1979 : Le jeu du morl, Ritologiques, 5, Paris, Aubier/Étranges/Étrangers, $278 \mathrm{p}$.

\section{MAHÉ N., SF́ciuitr M.}

1995 : L'établissement rural gallo-romain du chemin de Sens, in: Séguier M. (éd.), Un gisement archéologique de l'interfluve Seine-Yonne du Paléolithique. supérieur à l'Antiquité tardive à Marollessur-Seine, Document final de synthèse, Centre départemental de La Bassée, Service régional de l'archéologie d'île-de-France, Saint-Denis, p. 56-84.

\section{MANNIEZ Y}

1993 : Archéologie funéraire dans la cité de Bériers : la nécropole des Clapiès à Villeneuve-lès-Béziers (Hérault), in: Ferdière A. (dir.), Monde des morls, monde des vivants en Gaule rurale, Actes $\mathrm{du}$ colloque ARCHEA/AGER, Orléans, 7-9 févr. 1992, 6 e suppl. à la Revue archéologique du Centre de la France, p. 367-370.

MARTIN M.

1991 : Das spätrömisch-frühmiltelalterliche Gräberjeld von Kaiseraugst, Kanton Aargau, Derendingen, Habegger Verlag, 355 p.

Martin S., Blaizot F., Sarkasin J.-C., SAVINO V.

1994 : 'Travaux d'archéologie préventive sur le tracé de l'autoroule A 46 sud, rapport 
de fouille, Marennes Le Pillon (Rhône), Document final de synthèse, Service régional de l'Archéologie de Rhône-Alpes, Lyon, 75 p.

Masset C.

1975 : La mortalité préhistorique, Cahiers du Centre de recherches préhistoriques, 4 , p. 63-90.

1987 : Le " recrutement " d'un ensemble funéraire, in: Duday H., Masset C. (éds), Anthropologie physique et archéologie. Méthodes d'élude des sépultures, Paris, éd. Du CNRS, p. 111-134.

Masseit C., Selliler P.

1990 : Les anthropologues, les morts et les vivants, Les Nouvelles de l'Archéologie, 40, p. 5-8.

MONNII:R J.

2001 : Les établissements ruraux de l'Antiquité tardive en Suisse, in: Ouzoulias P., Pellecuer C., Raynaud C., Van Ossel P., Garmy P. (dir.), Les campagnes de la Gaule à la fin de l'Antiquité, Actes du IV" colloque de l'association AGER, Montpellier, 11-14 mars 1998, Antibes, éd. APDCA, p. 173-199.

MONNOYlLtr V.

1994 : La nécropole de la Côte à Ambronay (Ain), Document final de synthèse, Service régional de l'archéologie de Rhône-Alpes, Lyon, non paginé.

Moorrles C. F. A., Fanning; E. A., HunT E. E. JR

1963 : Formation and resorption of three deciduous teeth in children, American Journal of Physical Anthropology, 21, p. 205-213.

Morfi. G., Gruyer J.

1961 : Compte rendu el relevés de fouilles de la nécropole de la Boisse (Ain), Rapport de fouille, Service régional de l'archéologie de Rhône-Alpes, Lyon, non paginé.

MORRIS I.

1995 : About some theories de La nécropole antique, Topoï, 5, p. 295-302.
Ouzoulas P., Peilleculer C., Raynaud C., Van OsSel. P., Garmy P. (DIR.)

2001 : Les campagnes de la Gaule à la fin de l'Antiquité, Actes du IV' colloque de l'association AGER, Montpcllier, 11-14 mars 1998, Antibes, éd. APDCA.

Ouzoulins P., Van Ossil. P.

2001 : Dynamiques du peuplement et formes de l'habitat tardif: le cas de l'île-de-France, in: Ouzoulias P., Pellecuer C., Raynaud C., Van Ossel P., Garmy P. (dir.), Les campagnes de la Gaule à la fin de l'Antiquité, Actes du IV colloque de l'association AGER, Montpellier, 11-14 mars 1998, Antibes, éd. APDCA, p. 147-172.

OWincs-WebB P. A., Suchry J. M.

1985 : Epiphyseal union of the anterior iliac crest and medial clavicle in a modern multiracial sample of American males and females, American Journal of Anthropology, 68, p. 457-466.

Parodi A., Raynaud C., Roger J.-M.

1987 : La Vaunage du III" siècle au milieu du XII" siècle. Habitat et occupations des sols, Archéologie du Midi médiéval, V, p. 3-59.

Pritheculer C.

1986 : L'occupation gallo-romaine tardive autour du bassin de Thau, mémoire de DEA dactylographié, univ. d'Aixen-Provence.

Pirnon J., PERnon C.

1990 : Les potiers de Porloul. Productions, activités el cadre de vie d'un atelier au $V$ siècle cipr. J.-C. en Savoie, 20" suppl. à la Revue archéologique de Narbonnaise, $220 \mathrm{p}$.

Perraud R.

1974 : Le bris rituel aux I ${ }^{\mathrm{er}}$-II" siècles de notre ère d'après les fouilles des Plantées à Briord (Ain), Revue archéologique de l'Est et du Centre-Est, XXV, 1, p. 7-16.

Pétrequin A.-M., Pítrequin P., Castel. B., Chabeuf M., Chaix L.,
Fourniler G., Gaill.aRI) DE Semainville H., LuniströmBaudais K., Monnier J.-L., OdOU:E J.-L., PARISOT J.-P., Rrynaun C.

1980 : Le site funéraire de Soyria à Clairvaux-les-Lacs (Jura), Revue archéologique de l'Est et du Centre-Est, XXXI, 3-4, p. 157-230.

PHII.POTT R. A.

1993 : Late Roman cemetery organization in Britain, in: Struck M. (ed.), Römerzeilliche (iräber als Quellen zu Religion, Bevölkerungsstruktur und Sozialgeschichte, Actes du colloque de Mayence, 18-20 févr. 1991, Archäologische Schriften des Instituts für Vor- und Frühgeschichte der Johannes Gutenberg-Universität, Mainz, 3, p. 413-420.

P(omarèdels $\mathrm{H}$.

1995 : Saint-André-de-Codols, Nîmes, Gard, opération polyclinique Grand Sud, Document final de synthèse, Service régional de l'archéologie du Languedoc-Roussillon, Montpellier, avril 1995.

PORTE P.

1986 : Anthropologie historique et archéologie en Rhône-Alpes, in: Buchet L. (dir.), Le matériel anthropologique provenant des édifices religieux, Actes des $2^{c}$ journées anthropologiques de Valbonne, 6-8 juin 1983, Paris, éd. du CNRS, coll. Notes et monographies techniques, 19, p. $47-50$.

Pouyé B., Allouis M.-F., Bonifay M., Bouvill.: C., Calvet A., Lopr\%, A., LOPE\% C.

1994 : Une nécropole de l'Antiquité tardive à Cadarache (Saint-Paul-lèsDurance, Bouches-du-Rhône), Archéologie médiévale, 24, p. 51-135.

Prigint D., Hunot J.-L.

1996: La mort: voyage au pays des vivants. Pratiques funéraires en Anjou, Angers, $151 \mathrm{p}$.

Privati B.

1983 : La nécropole de Sézegnin, $N^{p}-V I I T^{P}$ s. 
Société d'histoire et d'archéologie de Genève, $181 \mathrm{p}$.

Prummel. W.

1992 : Early medieval doy burials among the Germanic tribes, Helinium, XXXII, 1-2, p. 132-194.

RATEL R.

1977 : La nécropole gallo-romaine de Gratte Dos, commune de Meuilley, Côte-d'Or, Rerue archéologique de l'Est et du Centre-Esl, XXVIII, 1-2, p. 63-98.

RAYNAUD C.

1987 : Typologie des sépultures et problèmes de datation. L'apport des fouilles de Lunel-Viel (Hérault), in: Raynaud C. (dir.), Nécropoles languedociennes de l'Antiquité tardive et du haut Moyen Âge, Archéologie en Languedoc, 4, p. 121-132.

1990 : Le village gallo-romain de Lunel-Viel (Héraull), Annales littéraires de l'université de Besançon, 422, Paris, Centre de recherches d'histoire ancienne, $97,353 \mathrm{p}$.

1996 : Les campagnes rhodaniennes: quelle crise?, in: Fiches J.-L. (dir.), Le III s. en Gaule Narbonnaise : données régionales sur la crise de l'Empire, Actes de la table ronde du GDR $954 \mathrm{du}$ CNRS, Aix-cn-Provence, La Baume, 15-16 sept. 1995, Sophia Antipolis, éd. APDCA, p. 189-212.

REYNAUD J.-F.

1998 : Lugnunum christianum, Lyon du IV au VIII s.: topographie, nécropoles et édifices religieux, Documents d'archéologie française, $69,280 \mathrm{p}$.

Ruchaud M., Bi.Anc A.

1963 : La nécropole de Fontager (Drôme), Cahiers rhodaniens, 10, Institut international d'études ligures, p. 83-88.

\section{ROMAN IMPERIAL COINAGE (RIC)}

1981 : RIC, The family of Constantine I (AD 337-364), in: Sutherland C. H. V., Litt D., Carso R. A. G., Mantingly H. (eds), Roman Imperial Coinage, VIII, London, Spink and Son Ltd, 605 p., $28 \mathrm{pl}$.
1984 : RIC/Valentinian -I- Theodosius, in : Sutherland C. H. V., Litt D., Carso R. A. G., Mantingly H. (eds), Roman Imperial Coinage, IX, London, Kent R. A. G., 334 p., 16 pl.

ROUGÉ D.

1993 : Détermination du sexe et de l'âge au décès des adultes à partir de restes osseux, thèse de Doctorat, Toulouse, Univ. Paul-Sabatier, $478 \mathrm{p}$.

SALADIN-D'ANGlure B.

1999 : Et Sapiens inventa l'homme, Documentaire Arte, 16.12.1999, $20 \mathrm{~h} 45$.

SAunders S. R., FitzGerald C., RoGers T., DudAR C., McKillop $\mathrm{H}$.

1992 : A test of several methods of skeletal age estimation using a documented archaeological sample, Canadian Sociely of Forensic Science, 25, 2, p. 97-117.

SCHULER R.

1995 : Nécropoles et sépultures d'époque romaine à Beauvais : état des connaissances, Revue archéologique de Picardie, 3-4, p. 49-140.

SCHWIITZER J.

1984 : L'habitat rural en Alsace au haut Moyen Âge, Riedisheim, J. Schweitzer, $270 \mathrm{p}$.

\section{SelLIER P.}

1987 : Les sépultures de Mehrgarh : de l'analyse ostéologique à la reconstitution du rituel funéraire, Annales Fyssen, 3, p. 17-35.

1989 : Hypotheses and estimators for the demographic interpretation of the. Chalcolithic population from Mehrgarh, Pakistan, East and West, 39, p. 11-42.

1996 : La mise en évidence d'anomalies démographiques et leur interprétation: population, recrutement et pratiques funéraires du tumulus de Courtesoult, in: Piningre J.-F. (éd.), Nécropoles et sociétés au premier âge du Fer: le tumulus de Courtesoult (HauteSaône), Documents d'archéologie française, 54, p. 188-200.
Selilier P., Castex D., Houẽt F.

1995 : Principes de distribution des classes d'âge non-adultes afin de mettre en évidence des anomalies démngraphiques dans les populations archéologiques, in : Chiarelli $\mathrm{B}$. (éd.), Biodémographie et évolution humaine, Préactes du colloque of International Union of Anthropological and Ethnological Science, Florence, 19-26 avril 1995, 2 p.

Steiner L., MENNa F.

2000 : La nécropole du Pré de la Cure à Yverdon-les-Bains (IV-VII s. apr. J.-C.), Cahiers d'archéologie romande, 7576, 1, Lausanne, $352 \mathrm{p}$.

STRUCK M.

1993 : Kinderbestattungen in romanobritischen Siedlungen - der archäologische Befund, in: Struck M. (ed.), Römerzeitliche Gräber als Quellen zu Religion, Bevölkerungsstruktur und Sozialgeschichte, Actes du colloque de Mayence, 18-20 févr. 1991, Archäologische Schriften des Instituts für Vor- und Frühgeschichte der Johannes Gutenberg-Universität, Mainz, 3, p. 313-318.

SUNDICK R. I.

1978 : Human skeletal growth and age determination, Homo, 29, p. 228-249.

SWIFT E.

1998 : Regional variation in personal ornaments (bracelets, beads, crosbow brooches, buckles and belt fittings) in the $4^{\text {th }}$ century in the North-Western provinces of the Roman Empire, in: Actualité de la recherche sur le mobilier romain non céramique, Communication du colloque de Chauvigny (86), 23-24 oct. 1998.

2000 : Regionality in dress accessories in the Late Roman West, Monographies Instrumentum, 11, Montagnac, $312 \mathrm{p}$.

TRANOY L.

1995 : Recherches sur les nécropoles antiques de Lyon: topographie et rites funéraires, 
thèse de Doctorat, univ. de ProvenceAix-Marseille I, déc. 1995, non publiée, 841 p., 220 fig.

Tranoy L. avec la collab. de Gisclon J.-L. 1995 : Le quai Arloing: artisanat et nécropole, in: Delaval E., Bellon C., Chastel J., Plassot E., Tranoy L. (éds), Vaise, un quartier de Lyon antique, Documents d'archéologie en RhôneAlpes, 11, p. 179-254.

UBELAKER D. H.

1978 : Human skeletal remains. Excavation, analysis, interpretation, Chicago, Aldine, $172 \mathrm{p}$.

\section{VAN OSSEL P.}

1992 : Établissements ruraux de l'Antiquité tardive dans le nord de la Gaule, $51^{\mathrm{e}}$ suppl. à Gallia, Paris, éd. du CNRS, $479 \mathrm{p}$.

1993 : L'occupation des campagnes dans le nord de la Gaule durant l'Antiquité tardive: l'apport des cimetières, in: Ferdière A. (dir.), Monde des morts, monde des vivants en Gaule rurale, Actes du colloque ARCHEA/AGER, Orléans, 7-9 févr. 1992, 6e suppl. à la Revue archéologique du Centre de la France, p. 185-196.

1997 : La part du Bas-Empire dans la formation de l'habitat rural du $\mathrm{VI}^{\mathrm{c}}$ siècle, in: Gauthier N., Galinié H. (éds), Grégoire de Tours et l'espace gaulois, Actes du congrès international de Tours, 3-5 nov. 1994, $13^{\mathrm{e}}$ suppl. à la Revue archéologique du Centre de la France, p. 81-91.

VICHERd G., BAUdRAND M.-N.

1982 : Fouilles récentes à La Boisse (Ain) du Bronze final au Bas-Empire, Revue archéologique de l'Est et du Centre-Est, XXXIII, 2-4, p. 123-138.

Vicherd G., MotTe S., Pichon M.

1985 : Le site du Recourbe à ChâteauGaillard dans l'Ain, Document final de synthèse, Service régional de l'archéologie de Rhône-Alpes, Lyon.

\section{WATTS D.}

1991 : Christians and Pagans in Roman Britain, London, Routledge, 277 p.

WIGG D.

1999 : Dieu, empereur et Victoire : le christianisme et la propagande monétaire dans l'Antiquité tardive, Dossiers d'archéologie, 248, nov., p. 84.

\section{WITTMANN A.}

2000 : Le mobilier céramique des sépultures, in: Alfonso G., Alix G., Blaizot F., Cabezuelo U., Lalaï D., Wittmann A. (éds), Beaumont (Puy-deDôme). Bassin d'orage amont de Pourlial, bâtiments annexes et nécropole d'immatures de la villa antique de Champ Madame, Document final de synthèse, Service régional de l'archéologie d'Auvergne, Clermont-Ferrand, p. $96-100$. 